SUPPORTING INFORMATION

\title{
Mechanistic Investigation into the Acetate-Initiated Catalytic Trimerization of Aliphatic Isocyanates: A Bicyclic Ride
}

\author{
Max Siebert, ${ }^{a}$ Rebecca Sure, ${ }^{b}$ Peter Deglmann, ${ }^{b}$ Anna C. Closs, ${ }^{a}$ \\ Frederic Lucas, ${ }^{b, *}$ and Oliver Trapp ${ }^{a, *}$ \\ ${ }^{a}$ Department Chemie, Ludwig-Maximilians-Universität München, \\ Butenandtstraße 5-13, 81377 München, Germany. \\ * Email: oliver.trapp@cup.uni-muenchen.de \\ ${ }^{b}$ Advanced Materials \& Systems Research, BASF SE, \\ Carl-Bosch-Straße 38, 67056 Ludwigshafen, Germany. \\ *Email: frederic.lucas@basf.com
}




\section{Contents}

1 Investigation of Pre-Catalyst Activation $\quad$ S4

2 Reference Compounds $\quad$ S7

2.1 NMR Spectra of Reference Compounds . . . . . . . . . . . . . . . . S S7

3 Reaction Monitoring via NMR Experiments $\quad$ S12

3.1 Reaction Monitoring via NMR with Internal Standard . . . . . . . . . . . . S12

3.1.1 ${ }^{1} \mathrm{H}$ NMR Spectra of Reaction Monitoring with Internal Standard . . S12

3.1.2 ${ }^{13}$ C NMR Spectra of Reaction Monitoring with Internal Standard . . S19

3.2 Reaction Monitoring via NMR without Internal Standard . . . . . . . . . . . S37

3.2.1 NMR Spectra of Reaction Monitoring without Internal Standard in a J. Young NMR Tube (Closed Atmosphere) . . . . . . . . . . . . . . . S37

3.2.2 NMR Spectra of Reaction Monitoring without Internal Standard in an NMR Tube (Open Atmosphere) . . . . . . . . . . . . . . . . . . . . S39

3.3 2D NMR Spectra of Final Catalysis Mixtures . . . . . . . . . . . . . . . . . S41

4 Analysis of Reaction Mixtures via Orbitrap-MS $\quad \mathbf{S 4 4}$

4.1 Analysis after Short Reaction Time $(15 \mathrm{~min}) \ldots \ldots$. . . . . . . . . . . . . . S44

4.2 Analysis after Complete Reaction . . . . . . . . . . . . . . . . . . S46

4.3 Overview of the Exact Masses of all Intermediates . . . . . . . . . . . . . . S48

5 Quantum Chemical Computations of Reaction Mechanisms S49

5.1 Overview of the Results . . . . . . . . . . . . . . . . . . . . . . . . S49

5.2 Cartesian Coordinates Catalytic Cycles . . . . . . . . . . . . . . . . S53

5.2.1 General Compounds . . . . . . . . . . . . . . . . . . . . S53

5.2 .2 Carboxylate Catalysis . . . . . . . . . . . . . . . S56

5.2 .3 Pre-Catalyst Activation . . . . . . . . . . . . . . . . . S66 
5.2.4 Deprotonated Amide Catalysis . . . . . . . . . . . . . . . . . . . . S67

5.2 .5 Catalyst Migration . . . . . . . . . . . . . . . . . . . . S87

5.2.6 Deprotonated Urea Catalysis . . . . . . . . . . . . . . . . . S104

5.3 Cartesian Coordinates Catalyst Decomposition . . . . . . . . . . . . . . . . . S129

5.3.1 General Compounds . . . . . . . . . . . . . . . . . . . . . . . . . S129

5.3 .2 Carboxylate Catalysis . . . . . . . . . . . . . . . . S132

5.3.3 Deprotonated Amide Catalysis . . . . . . . . . . . . . . . . . . . S138

5.3.4 Deprotonated Urea Catalysis . . . . . . . . . . . . . . . . . . S145

List of Abbreviations $\quad \$ 155$ 


\section{Investigation of Pre-Catalyst Activation}

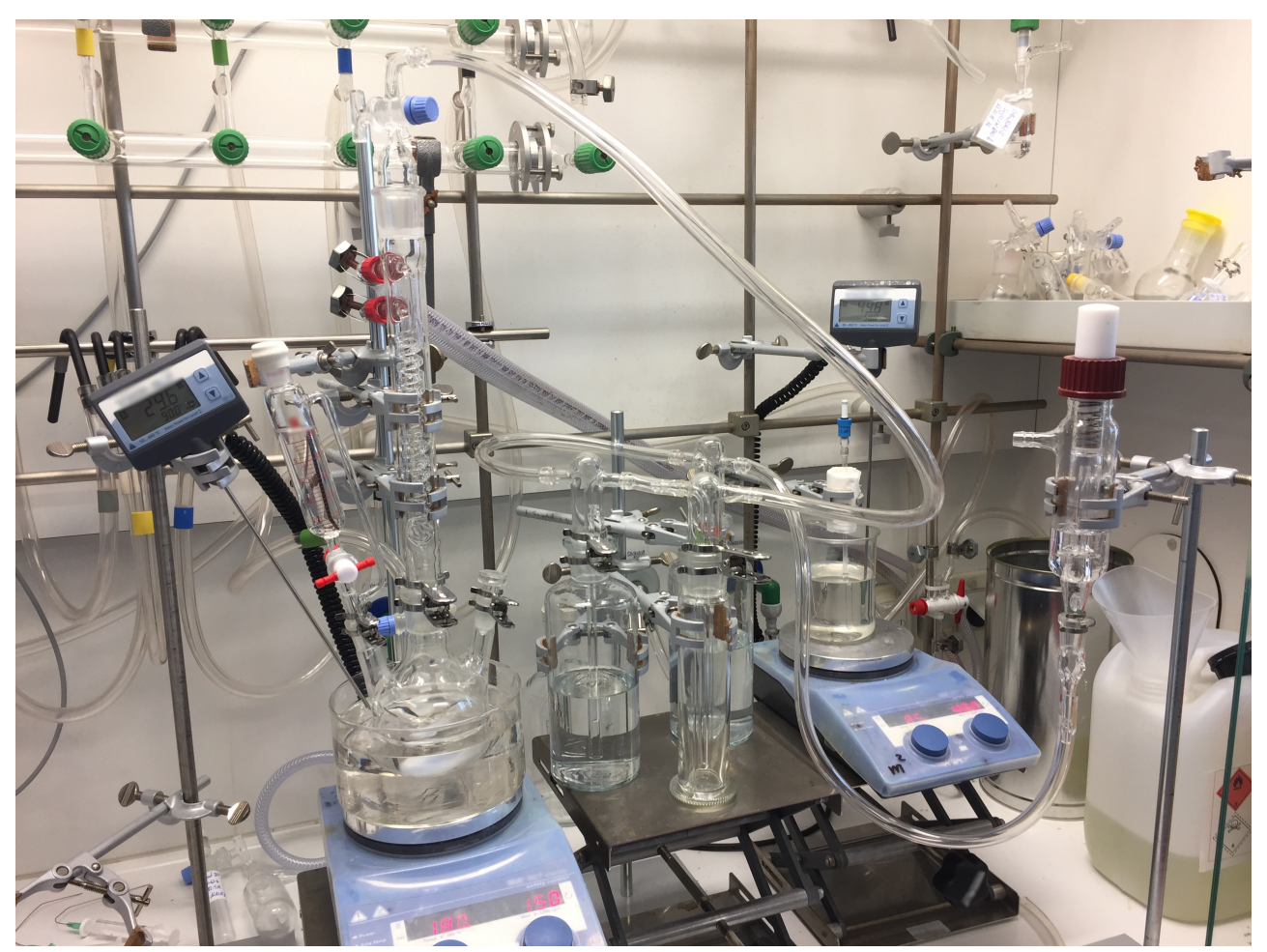

Figure S1: Reaction setup used to study the formation of $\mathrm{CO}_{2}$ during pre-catalyst activation. The three-neck Schlenk flask was equipped with a dropping funnel and a condenser. The Schlenk flask was sealed during reaction, so that potential gas had to pass through two gas washing bottles filled with baryta water. The inert setup was closed off with a pressure relief valve behind the gas washing bottles. 


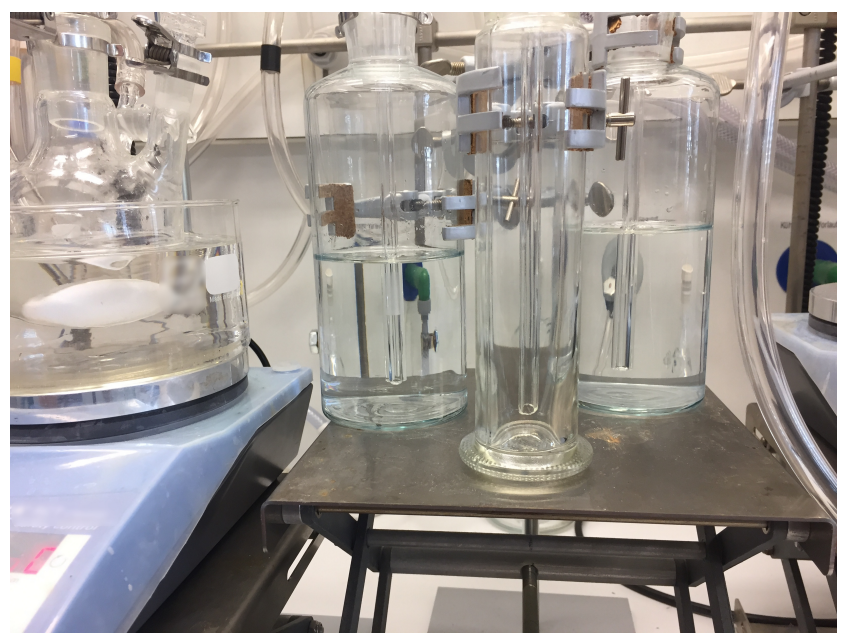

Figure S2: Close-up of the gas washing bottles with the clear barium hydroxide solution and the colorless catalyst solution in the Schlenk flask prior to isocyanate addition.

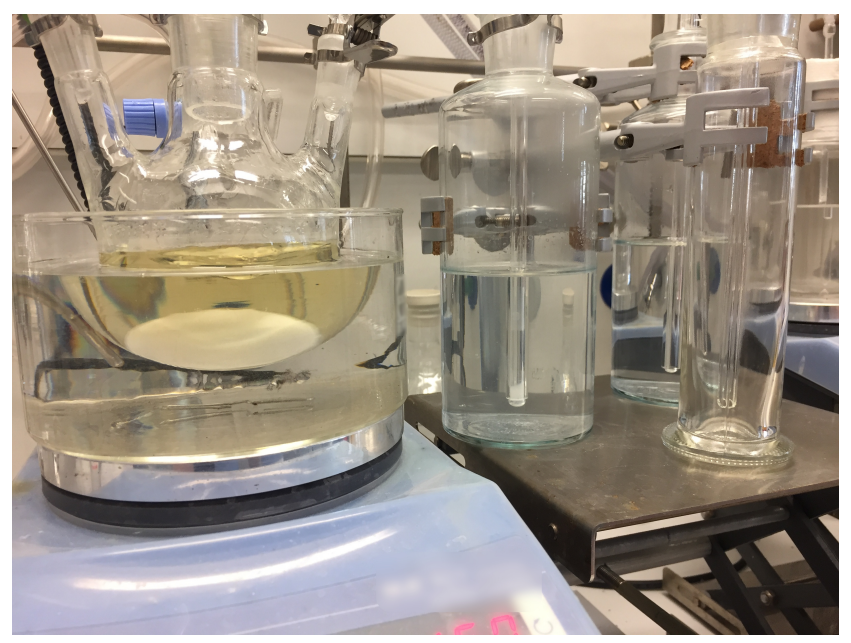

Figure S3: Close-up of the solution in the Schlenk flask that has turned yellow upon addition of the isocyanate solution and the gas washing bottles. The formation of bubbles in the gas washing bottles indicated the gas development during pre-catalyst activation. The barium hydroxide solution of the first gas washing bottle turned already slightly turbid. 


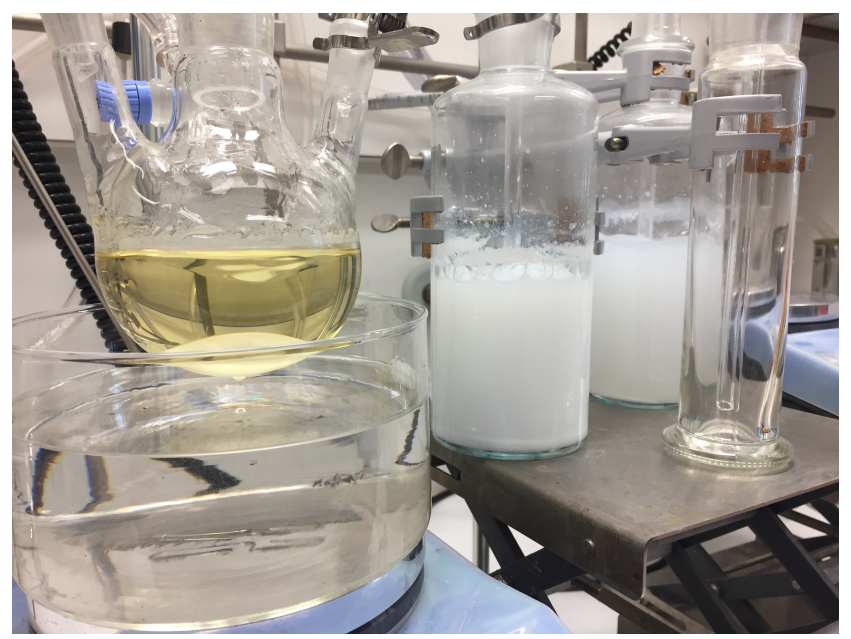

Figure S4: Close-up of the yellow solution in the Schlenk flask and the gas washing bottles. The formation of a milky suspension with high turbidity in the gas washing bottles proved the formation of large quantities of $\mathrm{CO}_{2}$ during pre-catalyst activation. 


\section{Reference Compounds}

\subsection{NMR Spectra of Reference Compounds}

\section{NMR Spectra of Acetate Catalyst 1}

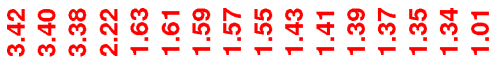

证

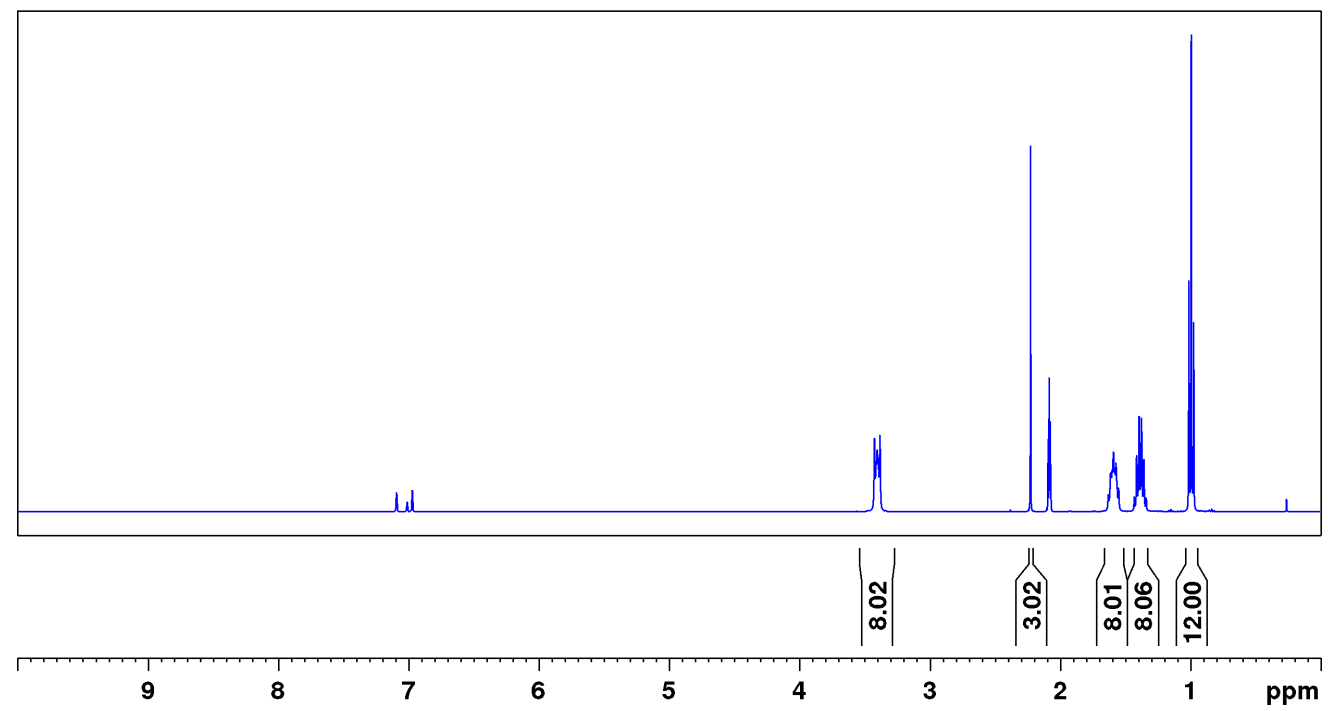

Figure S5: ${ }^{1} \mathrm{H}$ NMR (Toluene- $\mathrm{d}_{8}, 400 \mathrm{MHz}, 298 \mathrm{~K}$ ) spectrum of tetrabutylammonium acetate 1. 


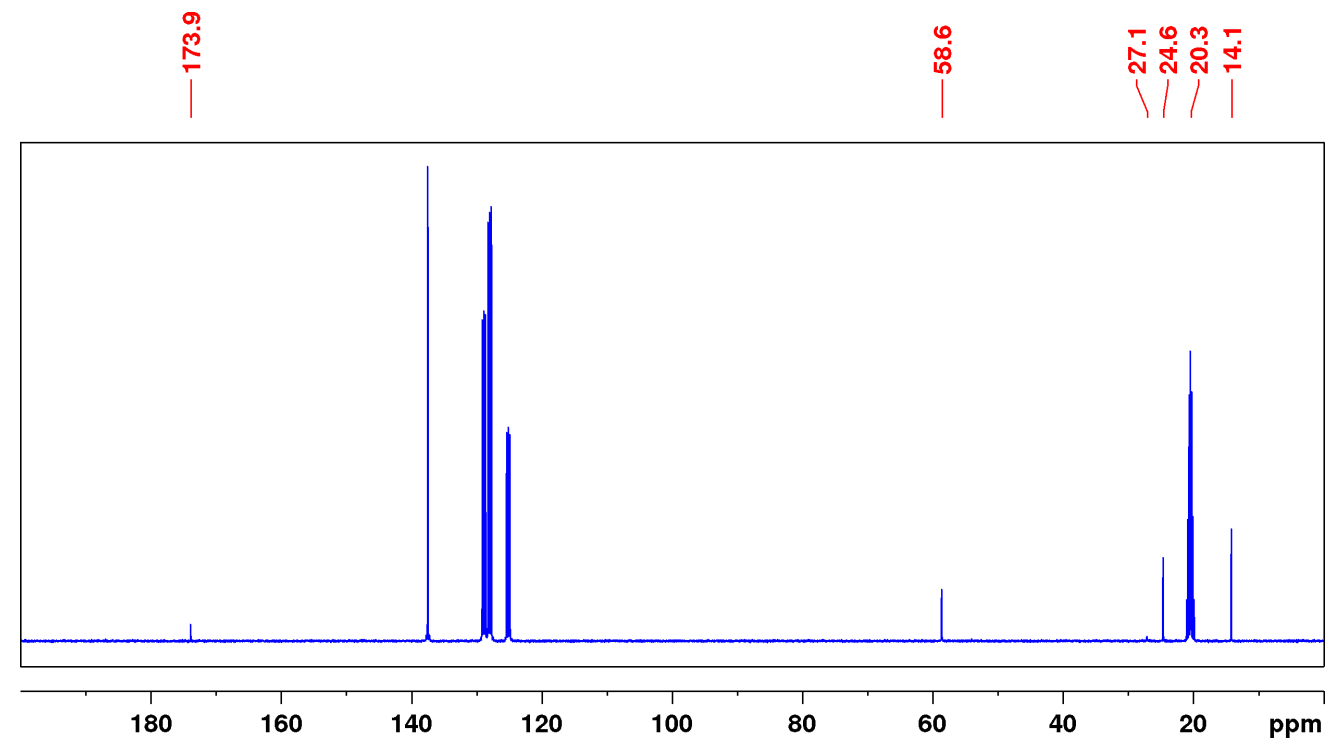

Figure S6: ${ }^{13} \mathrm{C}\left\{{ }^{1} \mathrm{H}\right\}$ NMR (Toluene-d $8,101 \mathrm{MHz}, 298 \mathrm{~K}$ ) spectrum of tetrabutylammonium acetate 1. 


\section{NMR Spectra of Isocyanate 2}

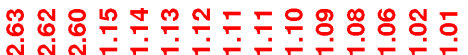
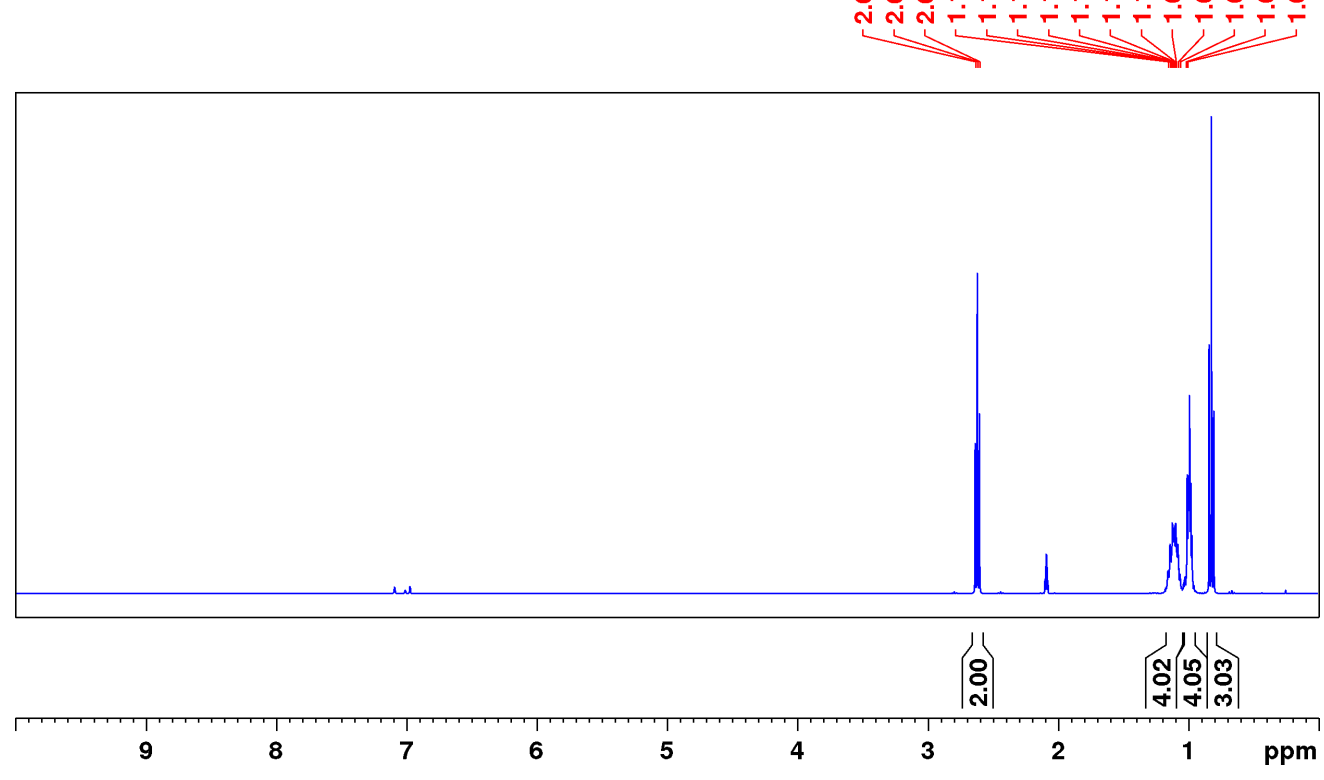

Figure S7: ${ }^{1} \mathrm{H}$ NMR (Toluene-d $, 400 \mathrm{MHz}, 298 \mathrm{~K}$ ) spectrum of $n$-hexyl isocyanate 2.

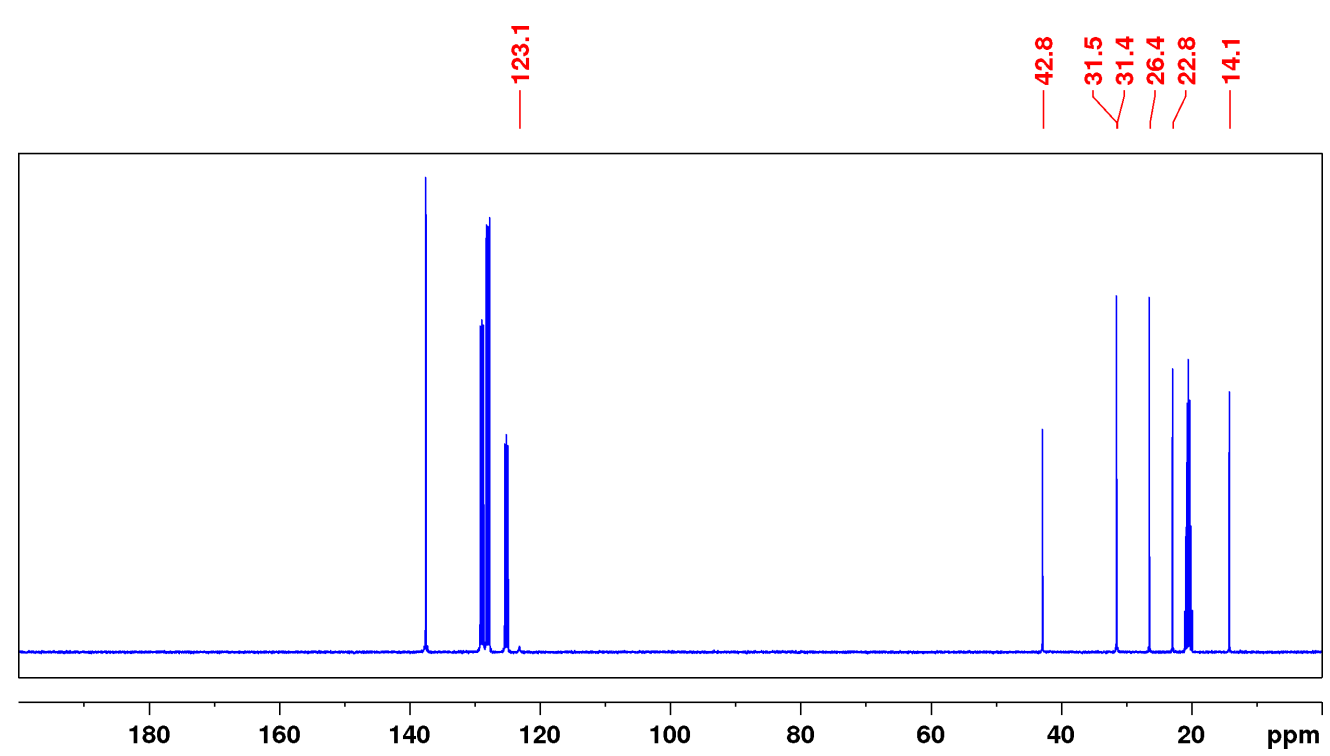

Figure S8: ${ }^{13} \mathrm{C}\left\{{ }^{1} \mathrm{H}\right\}$ NMR (Toluene-d $8,101 \mathrm{MHz}, 298 \mathrm{~K}$ ) spectrum of $n$-hexyl isocyanate 2. 


\section{NMR Spectra of Isocyanurate 6}

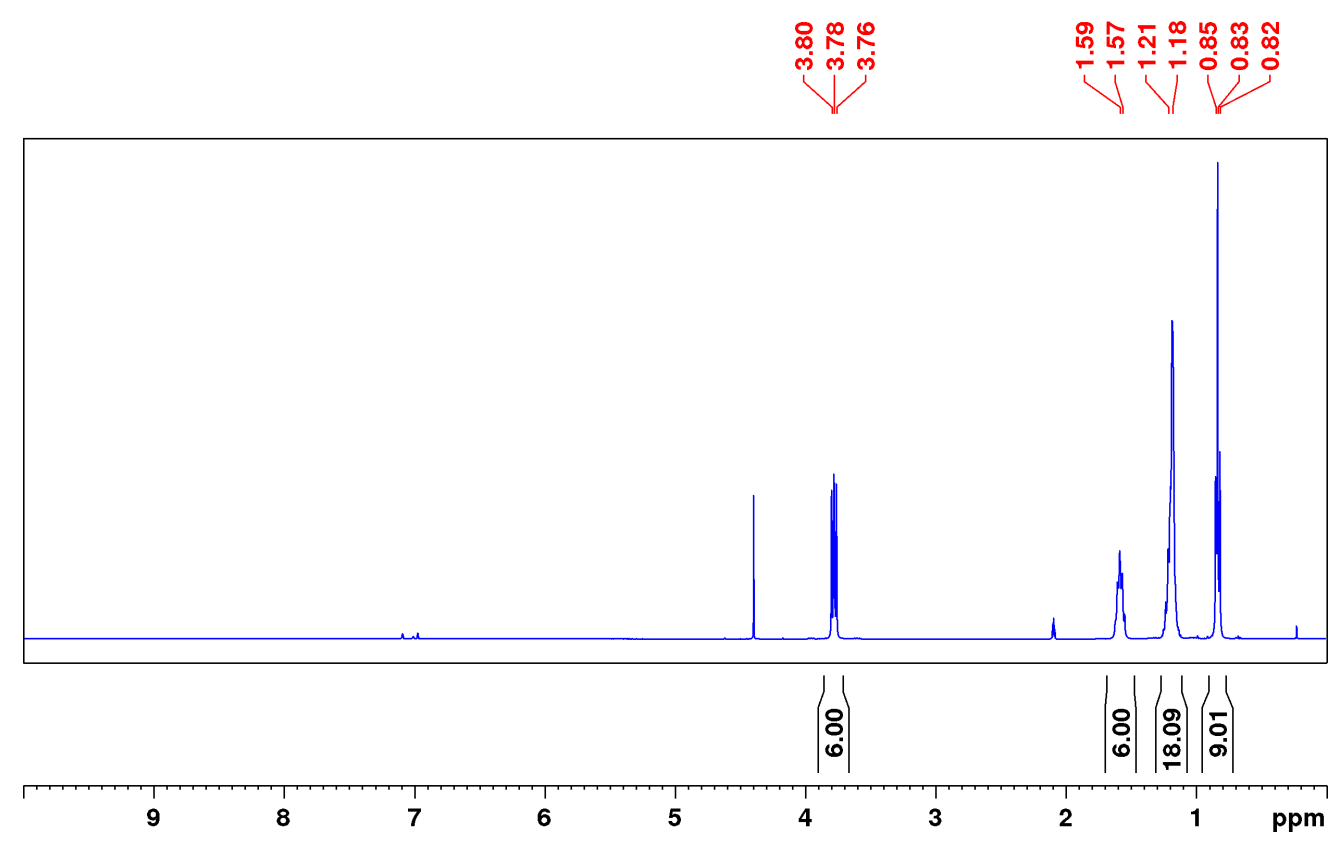

Figure S9: ${ }^{1} \mathrm{H}$ NMR (Toluene-d $8,400 \mathrm{MHz}, 298 \mathrm{~K}$ ) spectrum of $n$-hexyl isocyanurate 6.

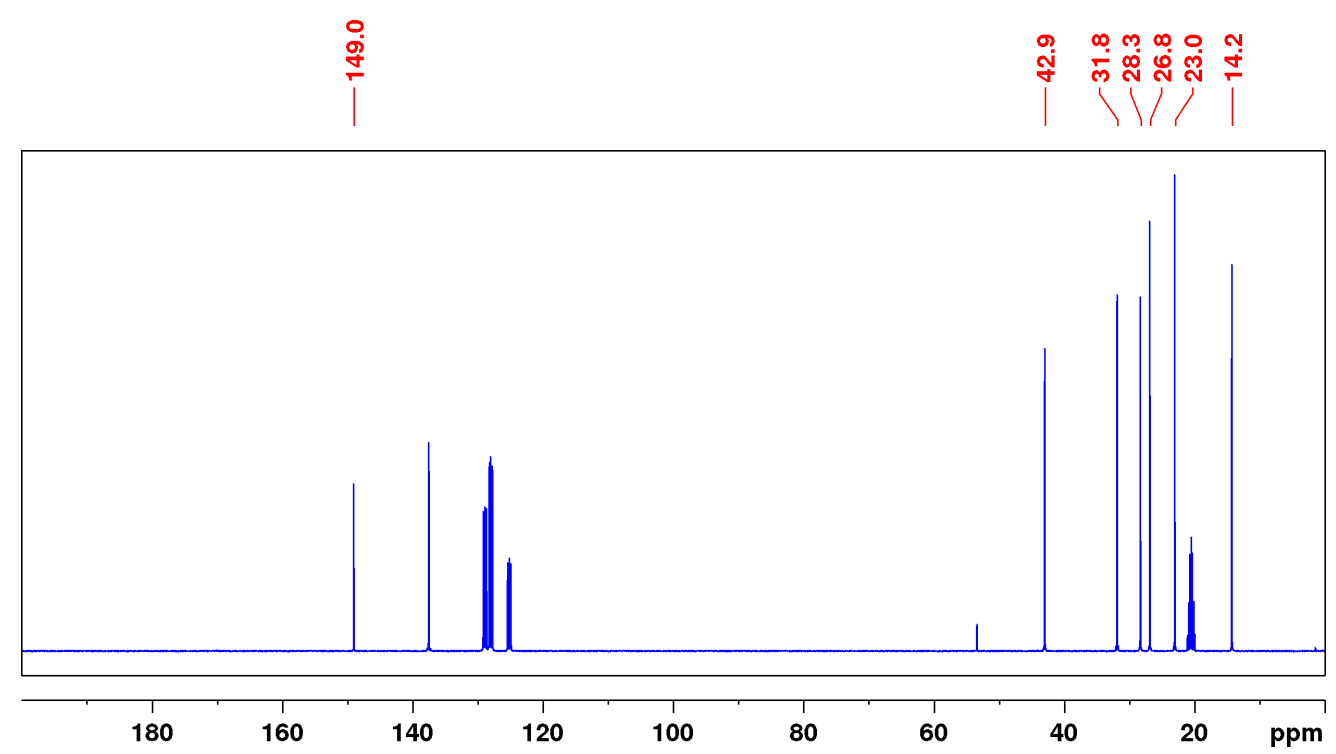

Figure S10: ${ }^{13} \mathrm{C}\left\{{ }^{1} \mathrm{H}\right\}$ NMR (Toluene-d $8,101 \mathrm{MHz}, 298 \mathrm{~K}$ ) spectrum of $n$-hexyl isocyanurate 6. 


\section{NMR Spectra of Uretdione 24}

心
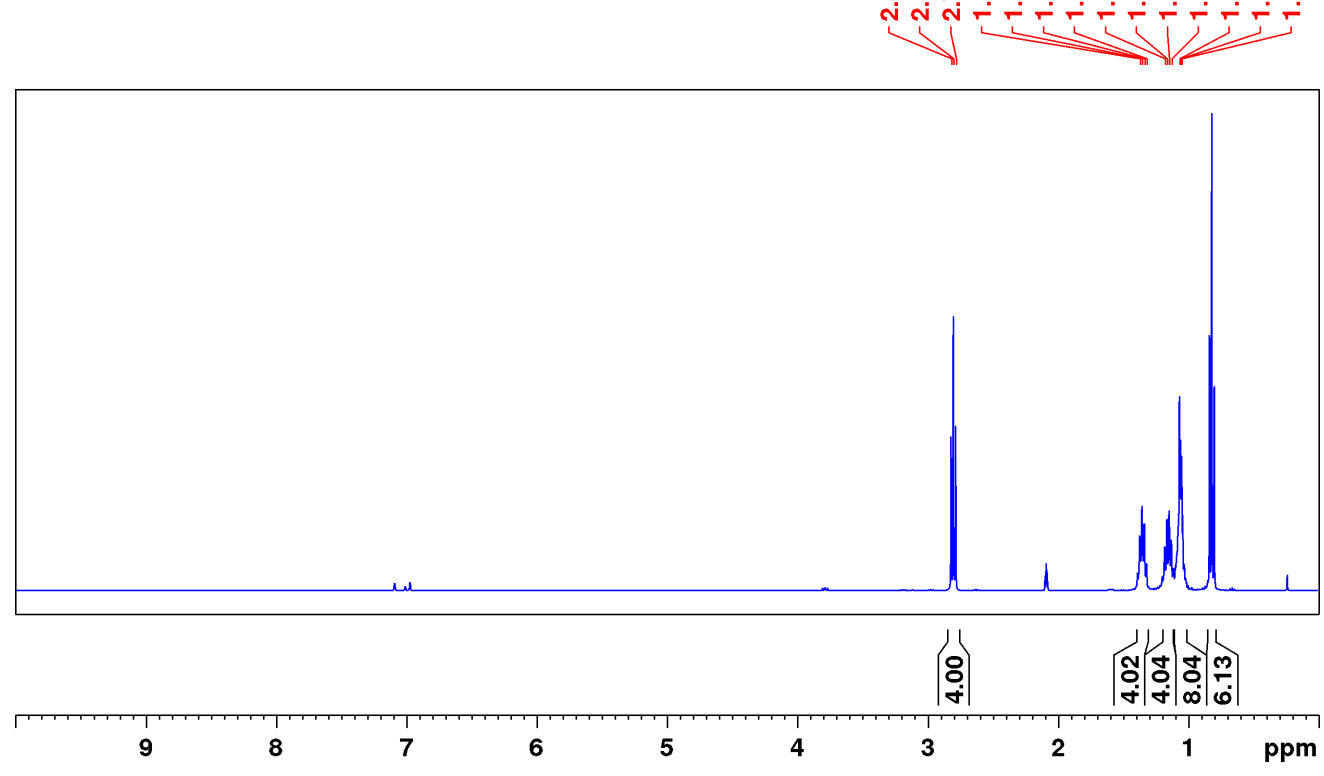

Figure S11: ${ }^{1} \mathrm{H}$ NMR (Toluene-d $8,400 \mathrm{MHz}, 298 \mathrm{~K}$ ) spectrum of $n$-hexyl uretdione 24.

$\stackrel{\infty}{\stackrel{\infty}{n}}$

นִำ

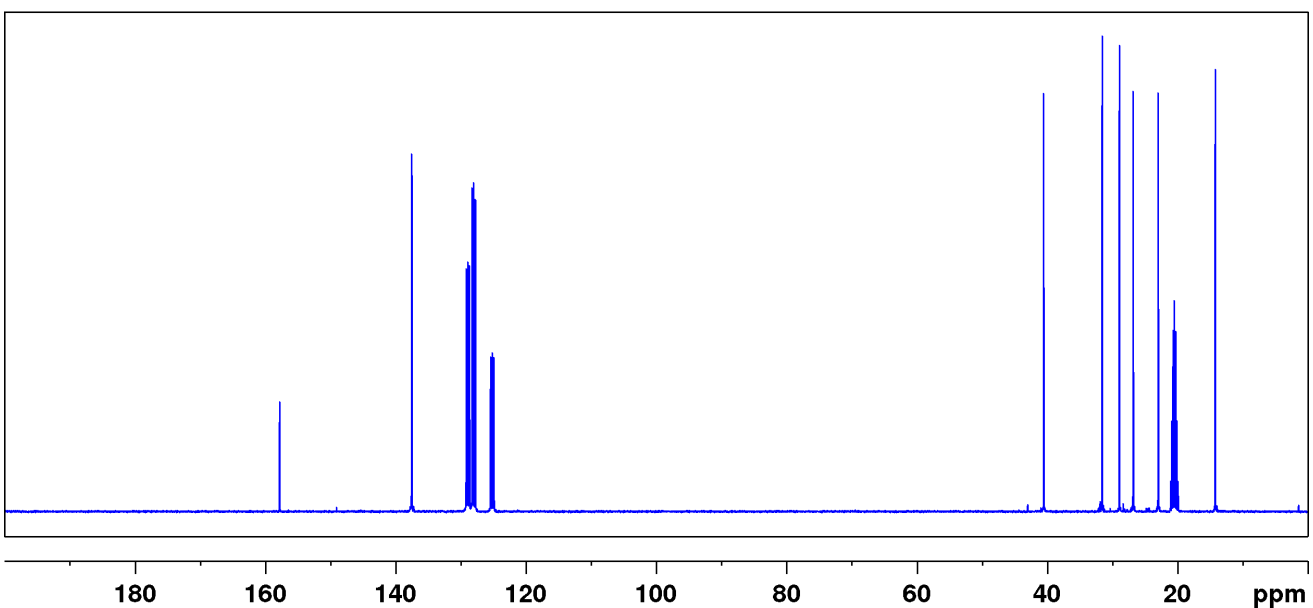

Figure S12: ${ }^{13} \mathrm{C}\left\{{ }^{1} \mathrm{H}\right\}$ NMR (Toluene-d $, 101 \mathrm{MHz}, 298 \mathrm{~K}$ ) spectrum of $n$-hexyl uretdione 24 


\section{Reaction Monitoring via NMR Experiments}

\subsection{Reaction Monitoring via NMR with Internal Standard}

\subsection{1 ${ }^{1} \mathrm{H}$ NMR Spectra of Reaction Monitoring with Internal Standard}

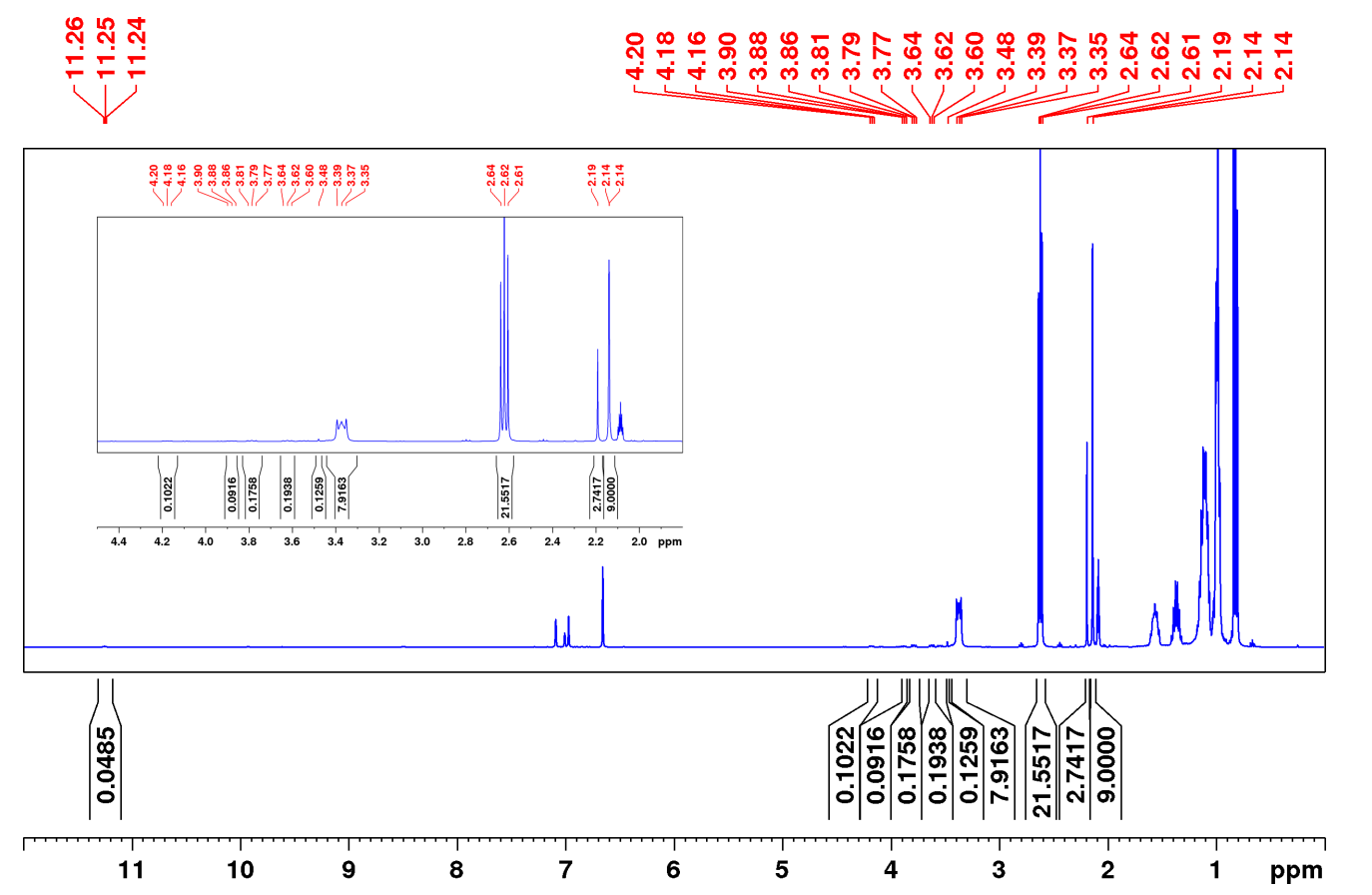

Figure S13: ${ }^{1} \mathrm{H}$ NMR (Toluene-d $, 400 \mathrm{MHz}, 298 \mathrm{~K}$ ) spectrum of a reaction mixture in a J. Young NMR tube after 0 min at $80^{\circ} \mathrm{C}$. Mesitylene $(18 \mu \mathrm{mol})$ was used as internal standard. 


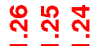

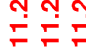

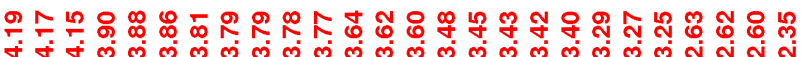

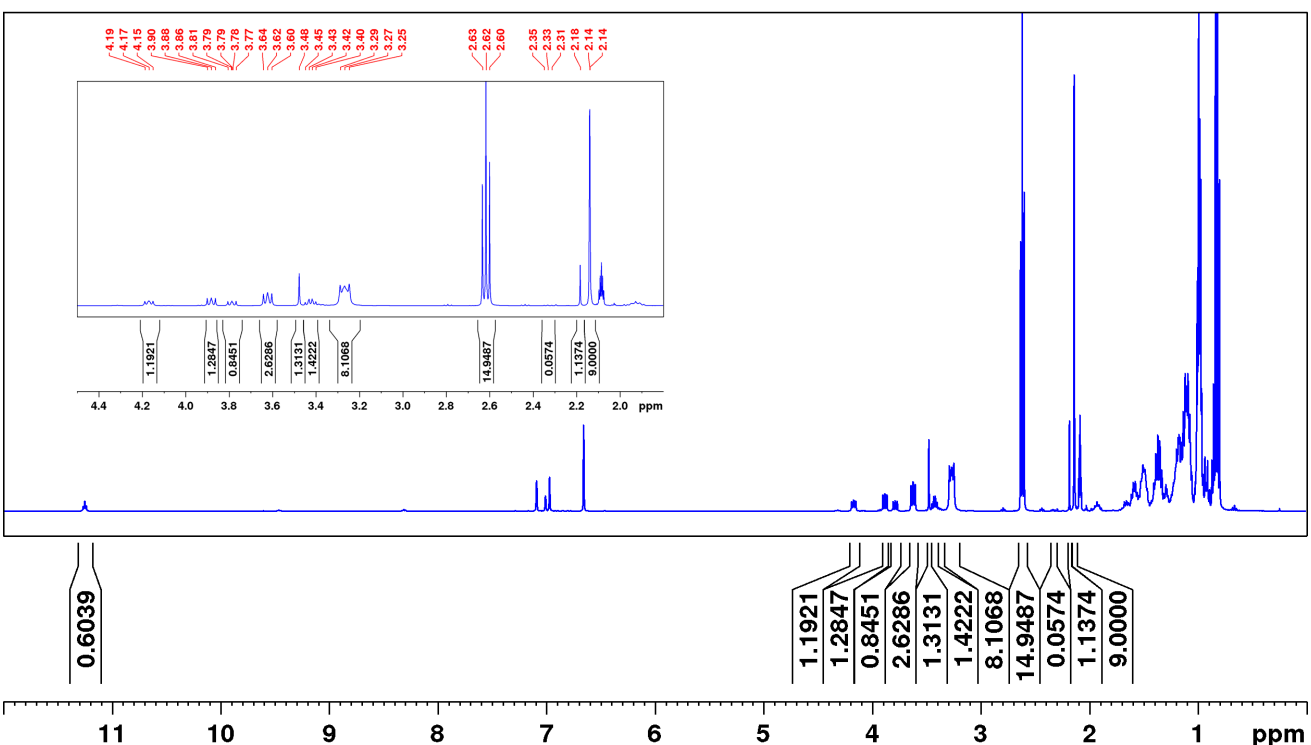

Figure S14: ${ }^{1} \mathrm{H}$ NMR (Toluene- $\mathrm{d}_{8}, 400 \mathrm{MHz}, 298 \mathrm{~K}$ ) spectrum of a reaction mixture in a J. Young NMR tube after 5 min at $80^{\circ} \mathrm{C}$. Mesitylene $(18 \mu \mathrm{mol})$ was used as internal standard.

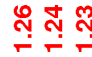

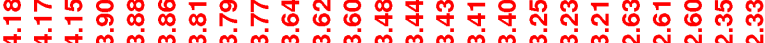

i

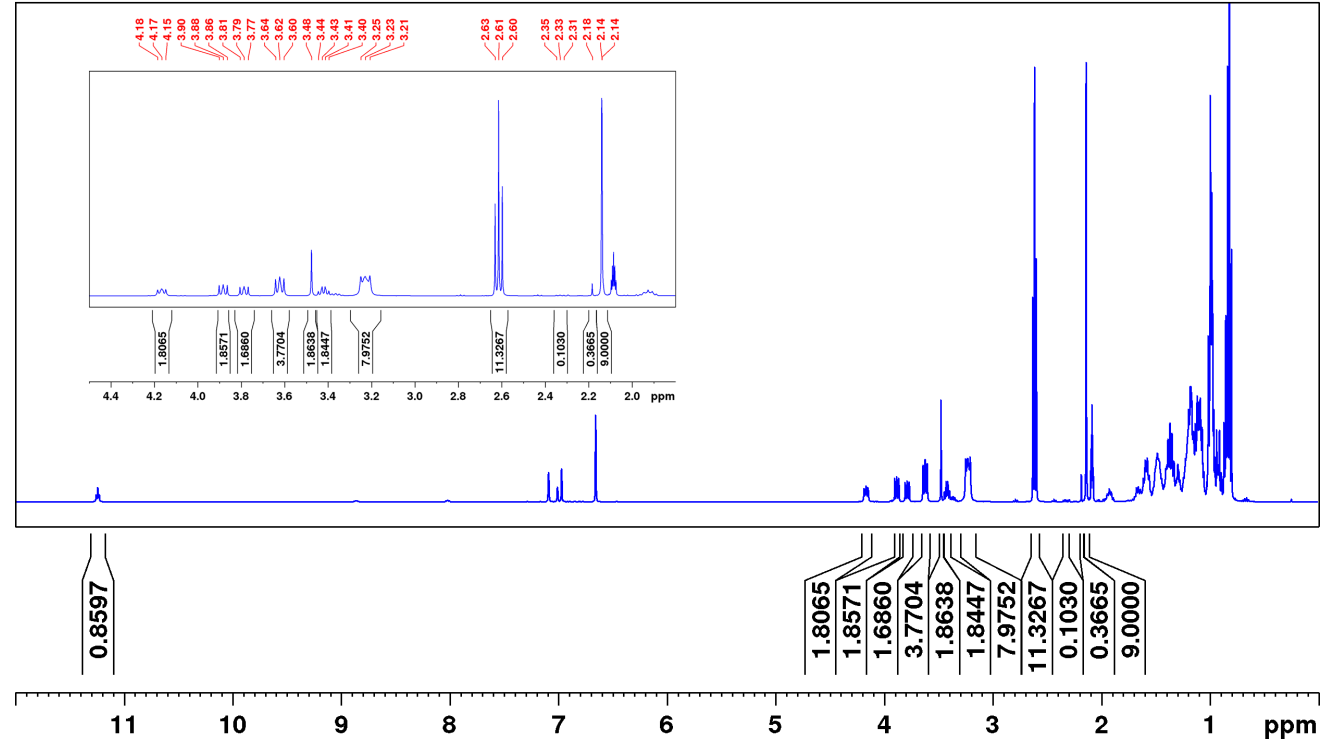

Figure S15: ${ }^{1} \mathrm{H}$ NMR (Toluene- $\mathrm{d}_{8}, 400 \mathrm{MHz}, 298 \mathrm{~K}$ ) spectrum of a reaction mixture in a J. Young NMR tube after 15 min at $80^{\circ} \mathrm{C}$. Mesitylene $(18 \mu \mathrm{mol})$ was used as internal standard. 


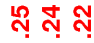

$F=$

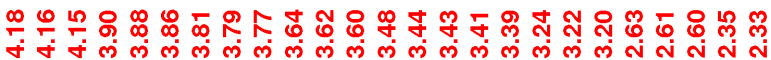

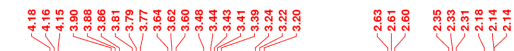
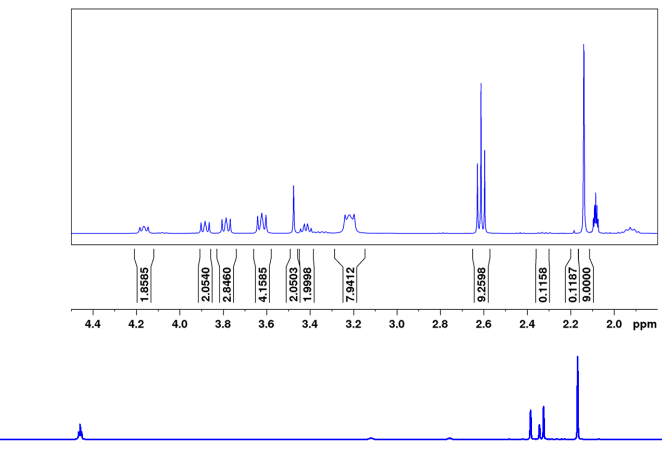

wollal
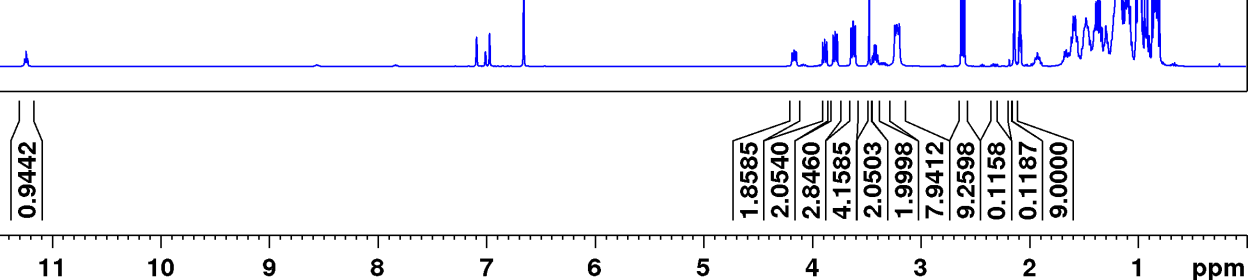

Figure S16: ${ }^{1} \mathrm{H}$ NMR (Toluene- $\mathrm{d}_{8}, 400 \mathrm{MHz}, 298 \mathrm{~K}$ ) spectrum of a reaction mixture in a J. Young NMR tube after 30 min at $80^{\circ} \mathrm{C}$. Mesitylene $(18 \mu \mathrm{mol})$ was used as internal standard.

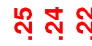

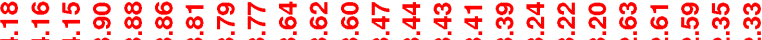

단

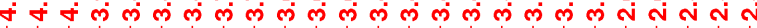

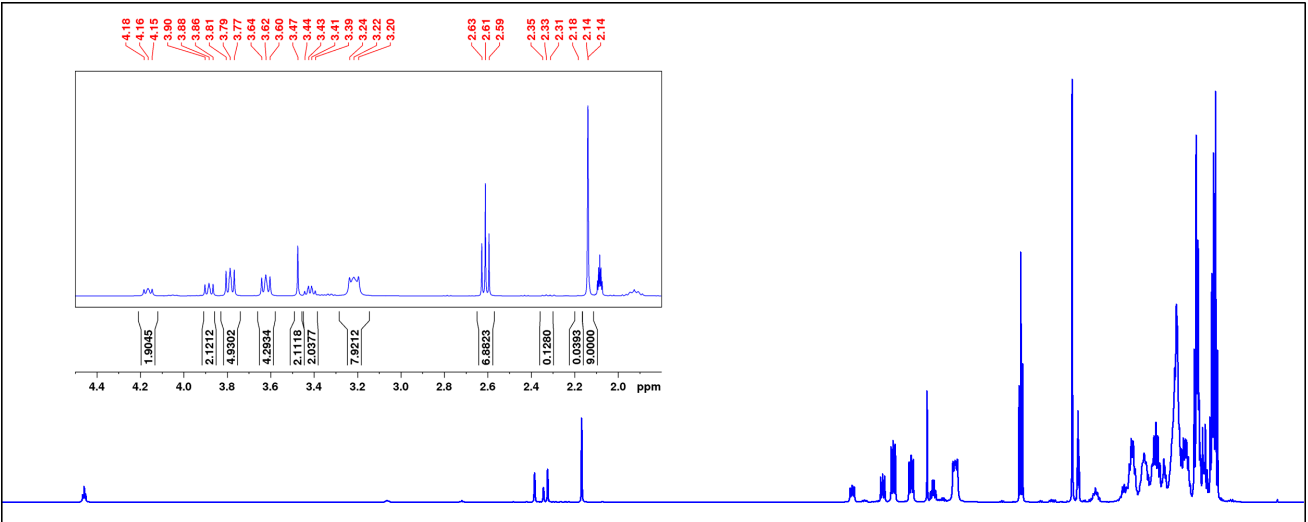

용
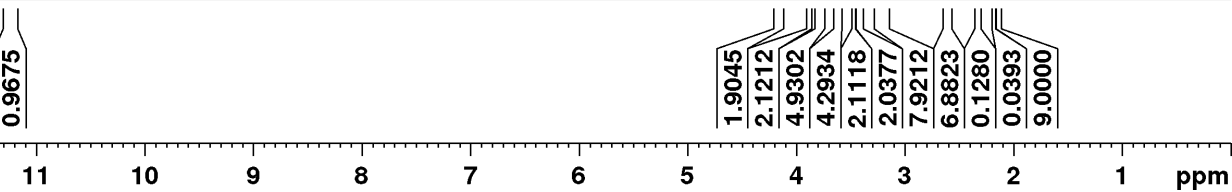

Figure S17: ${ }^{1} \mathrm{H}$ NMR (Toluene- $\mathrm{d}_{8}, 400 \mathrm{MHz}, 298 \mathrm{~K}$ ) spectrum of a reaction mixture in a J. Young NMR tube after $60 \mathrm{~min}$ at $80^{\circ} \mathrm{C}$. Mesitylene $(18 \mu \mathrm{mol})$ was used as internal standard. 


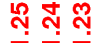

$F=$

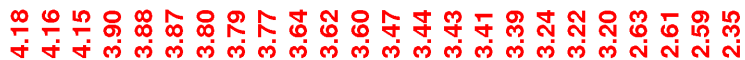

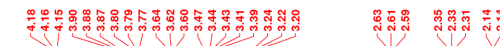
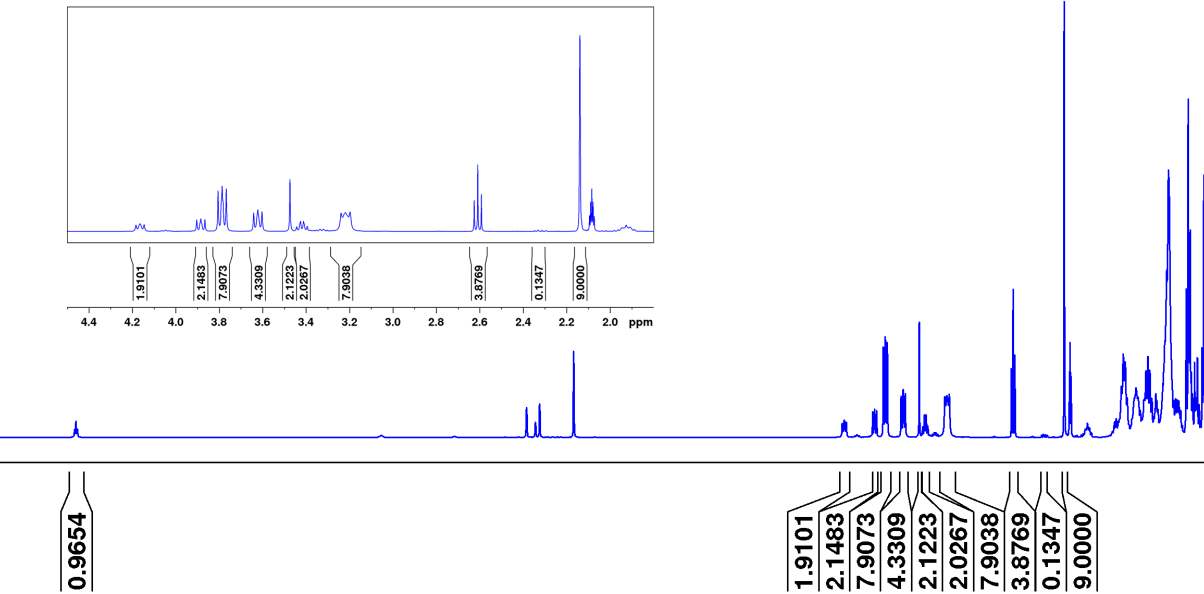

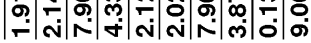

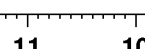

9

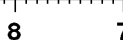

6

5

4

Figure S18: ${ }^{1} \mathrm{H}$ NMR (Toluene- $\mathrm{d}_{8}, 400 \mathrm{MHz}, 298 \mathrm{~K}$ ) spectrum of a reaction mixture in a J. Young NMR tube after 120 min at $80^{\circ} \mathrm{C}$. Mesitylene $(18 \mu \mathrm{mol})$ was used as internal standard.

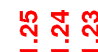

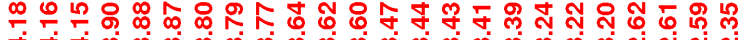

लें ले

ij

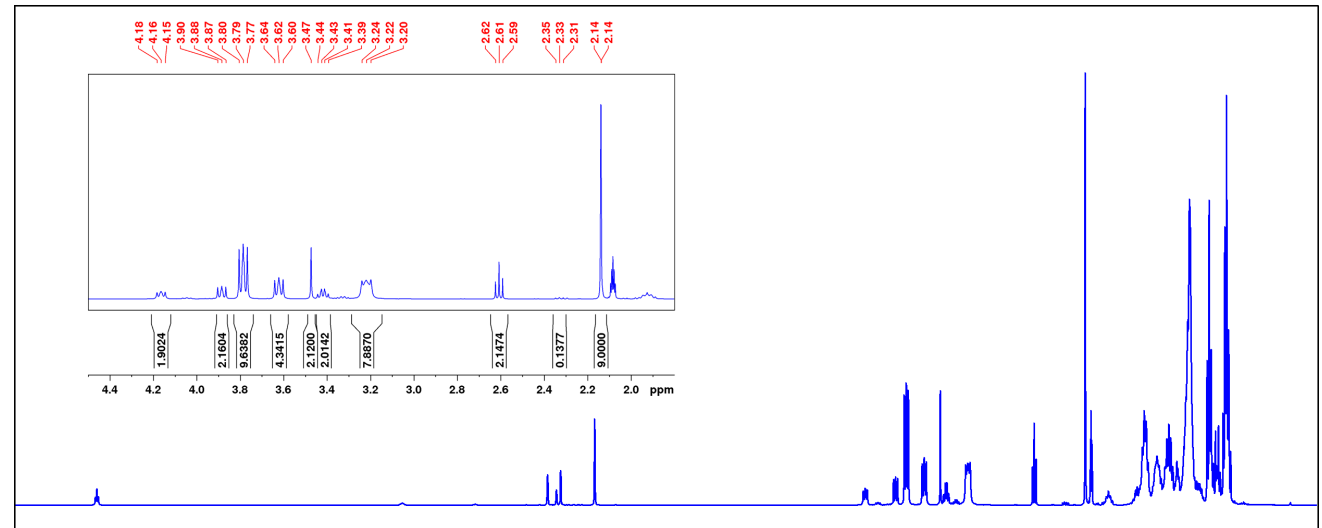

|ำ

ลับำ

Oִ

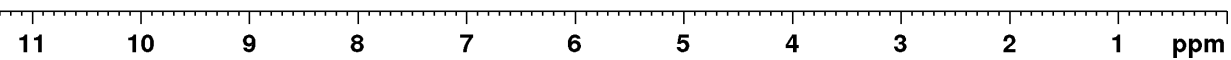

Figure S19: ${ }^{1} \mathrm{H}$ NMR (Toluene- $\mathrm{d}_{8}, 400 \mathrm{MHz}, 298 \mathrm{~K}$ ) spectrum of a reaction mixture in a J. Young NMR tube after 180 min at $80^{\circ} \mathrm{C}$. Mesitylene $(18 \mu \mathrm{mol})$ was used as internal standard. 


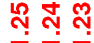

$\dot{\Gamma}+\frac{100}{10}$

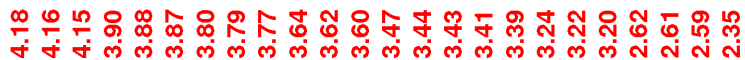

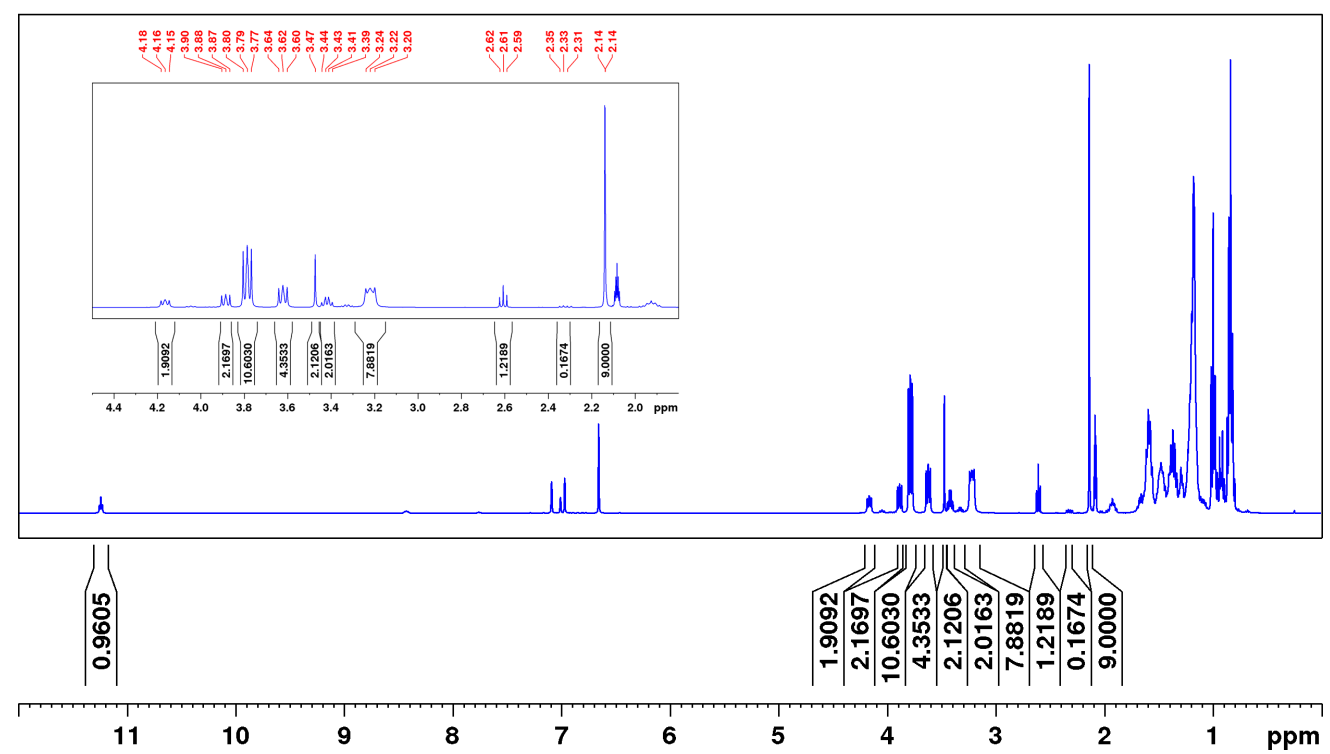

Figure S20: ${ }^{1} \mathrm{H}$ NMR (Toluene- $\mathrm{d}_{8}, 400 \mathrm{MHz}, 298 \mathrm{~K}$ ) spectrum of a reaction mixture in a J. Young NMR tube after $240 \mathrm{~min}$ at $80^{\circ} \mathrm{C}$. Mesitylene $(18 \mu \mathrm{mol})$ was used as internal standard.

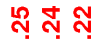

$\stackrel{i}{F}$

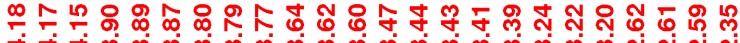

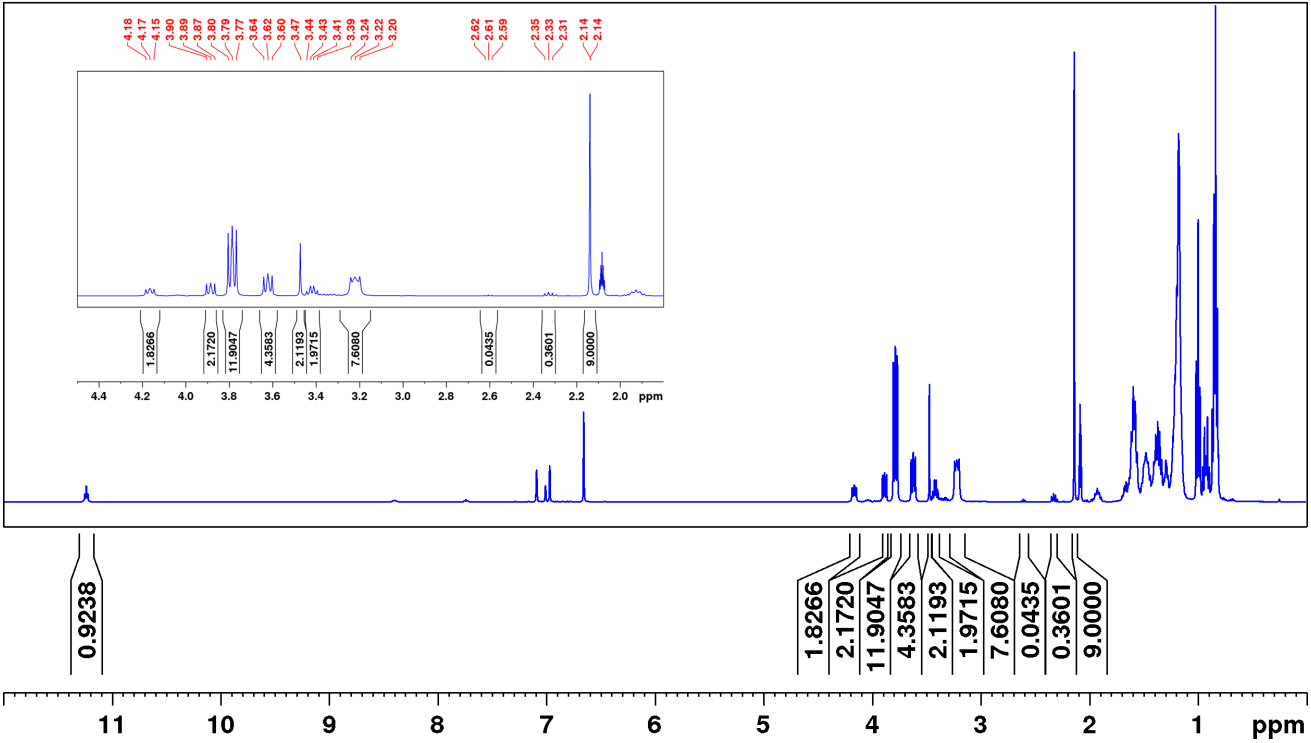

Figure S21: ${ }^{1} \mathrm{H}$ NMR (Toluene-d $\mathrm{d}_{8}, 400 \mathrm{MHz}, 298 \mathrm{~K}$ ) spectrum of a reaction mixture in a J. Young NMR tube after $1155 \mathrm{~min}$ at $80^{\circ} \mathrm{C}$. Mesitylene $(18 \mu \mathrm{mol})$ was used as internal standard. 


\section{Overview of the Reaction Monitoring}

All data available from the ${ }^{1} \mathrm{H}$ NMR spectra including integrals and respective concentrations is given in Table S1. 
Table S1: Overview of integrals from the ${ }^{1} \mathrm{H}$ NMR spectra for all relevant species and their respective concentrations.

\begin{tabular}{|c|c|c|c|c|c|c|c|c|c|c|c|c|c|}
\hline \multirow{2}{*}{$\begin{array}{r}\text { Molecule: } \\
\text { Group: }\end{array}$} & \multirow{2}{*}{$\begin{array}{c}\mathbf{I S}^{a} \\
\mathrm{CH}_{3}\end{array}$} & \multirow{2}{*}{$\frac{\mathbf{R}-\mathrm{NCO}}{\alpha-\mathrm{CH}_{2}}$} & \multirow{2}{*}{$\frac{\text { Trimer }}{\alpha-\mathrm{CH}_{2}}$} & \multirow{2}{*}{$\frac{\text { Acetate }}{\mathrm{CH}_{3}}$} & \multirow{2}{*}{$\frac{\mathrm{NBu}_{4}{ }^{+}}{\alpha-\mathrm{CH}_{2}}$} & \multirow{2}{*}{$\frac{\mathrm{NBu}_{3}{ }^{b}}{\alpha-\mathrm{CH}_{2}}$} & \multicolumn{3}{|c|}{ Olefin } & \multicolumn{3}{|c|}{ Amidocarbamate } & \multirow[b]{5}{*}{ Sum } \\
\hline & & & & & & & $\mathrm{sp}^{2}-\mathrm{CH}_{2}$ & $\alpha-\mathrm{CH}_{2}$ & $\alpha-\mathrm{CH}_{2}$ & $\alpha-\mathrm{CH}_{2}$ & $\alpha-\mathrm{CH}_{2}$ & $\mathrm{NH}$ & \\
\hline Integral: & $9 \mathrm{H}$ & $2 \mathrm{H}$ & $6 \mathrm{H}$ & $3 \mathrm{H}$ & $8 \mathrm{H}$ & $6 \mathrm{H}$ & $2 \mathrm{H}$ & $4 \mathrm{H}$ & $2 \mathrm{H}$ & $2 \mathrm{H}$ & $2 \mathrm{H}$ & $1 \mathrm{H}$ & \\
\hline $\begin{array}{l}\text { Center } \\
\text { (ppm): }\end{array}$ & 2.139 & $\begin{array}{l}2.620- \\
2.605\end{array}$ & 3.785 & $\begin{array}{l}2.190- \\
2.183\end{array}$ & $\begin{array}{l}3.372- \\
3.220\end{array}$ & 2.330 & $\begin{array}{l}3.479- \\
3.473\end{array}$ & $\begin{array}{l}3.623- \\
3.620\end{array}$ & $\begin{array}{l}3.880- \\
3.885\end{array}$ & $\begin{array}{l}3.425- \\
3.418\end{array}$ & $\begin{array}{l}4.175- \\
4.165\end{array}$ & $\begin{array}{c}11.2475- \\
11.2375\end{array}$ & \\
\hline \multirow[t]{2}{*}{$\begin{array}{l}\text { Width } \\
\text { (ppm): }\end{array}$} & 0.050 & 0.080 & 0.090 & $\begin{array}{l}0.040- \\
0.034\end{array}$ & 0.140 & 0.060 & $\begin{array}{l}0.028- \\
0.035\end{array}$ & $\begin{array}{l}0.065- \\
0.080\end{array}$ & $\begin{array}{c}0.048- \\
0.050\end{array}$ & 0.065 & 0.090 & 0.135 & \\
\hline & \multicolumn{13}{|c|}{ Integrals } \\
\hline $0 \mathrm{~min}$ & 9.0000 & 21.5517 & 0.1758 & 2.7417 & 7.9163 & 0.0000 & 0.1259 & 0.1938 & 0.0916 & n.d..$^{e}$ & 0.1022 & 0.0485 & 41.9475 \\
\hline $5 \mathrm{~min}$ & 9.0000 & 14.9487 & 0.8451 & 1.1374 & 8.1068 & 0.0517 & 1.3131 & 2.6286 & 1.2847 & 1.4222 & 1.1921 & 0.6039 & 42.5917 \\
\hline $15 \mathrm{~min}$ & 9.0000 & 11.3267 & 1.6860 & 0.3665 & 7.9752 & 0.1012 & 1.8638 & 3.7704 & 1.8571 & 1.8447 & 1.8065 & 0.8597 & 42.5608 \\
\hline $30 \mathrm{~min}$ & 9.0000 & 9.2598 & 2.8460 & 0.1187 & 7.9412 & 0.1152 & 2.0530 & 4.1585 & 2.0540 & 1.9998 & 1.8585 & 0.9442 & 42.4620 \\
\hline $60 \mathrm{~min}$ & 9.0000 & 6.8823 & 4.9302 & 0.0393 & 7.9212 & 0.1278 & 2.1118 & 4.2934 & 2.1212 & 2.0377 & 1.9045 & 0.9675 & 42.4649 \\
\hline $120 \mathrm{~min}$ & 9.0000 & 3.8769 & 7.9073 & 0.0000 & 7.9038 & 0.1347 & 2.1223 & 4.3309 & 2.1483 & 2.0267 & 1.9101 & 0.9654 & 42.4611 \\
\hline $180 \mathrm{~min}$ & 9.0000 & 2.1474 & 9.6382 & 0.0000 & 7.8870 & 0.1377 & 2.1200 & 4.3415 & 2.1604 & 2.0142 & 1.9024 & 0.9616 & 42.4481 \\
\hline $240 \mathrm{~min}$ & 9.0000 & 1.2189 & 10.6030 & 0.0000 & 7.8819 & 0.1674 & 2.1206 & 4.3533 & 2.1697 & 2.0163 & 1.9092 & 0.9605 & 42.5682 \\
\hline \multirow[t]{2}{*}{$1155 \mathrm{~min}$} & 9.0000 & 0.0435 & 11.9047 & 0.0000 & 7.6080 & 0.3601 & 2.1193 & 4.3583 & 2.1720 & 1.9715 & 1.8266 & 0.9238 & 42.6479 \\
\hline & \multicolumn{13}{|c|}{ Concentration $(\mathrm{mol} / \mathrm{L})$} \\
\hline 0 min & 0.0360 & 0.3879 & 0.0011 & 0.0329 & 0.0356 & 0.0000 & 0.0023 & 0.0017 & 0.0016 & n.d..$^{e}$ & 0.0018 & 0.0017 & \\
\hline $5 \mathrm{~min}$ & 0.0360 & 0.2691 & 0.0051 & 0.0136 & 0.0365 & 0.0003 & 0.0236 & 0.0237 & 0.0231 & 0.0256 & 0.0215 & 0.0217 & \\
\hline $15 \mathrm{~min}$ & 0.0360 & 0.2039 & 0.0101 & 0.0044 & 0.0359 & 0.0006 & 0.0335 & 0.0339 & 0.0334 & 0.0332 & 0.0325 & 0.0309 & \\
\hline $30 \mathrm{~min}$ & 0.0360 & 0.1667 & 0.0171 & 0.0014 & 0.0357 & 0.0007 & 0.0369 & 0.0374 & 0.0370 & 0.0360 & 0.0335 & 0.0340 & \\
\hline $60 \mathrm{~min}$ & 0.0360 & 0.1239 & 0.0296 & 0.0005 & 0.0356 & 0.0008 & 0.0380 & 0.0386 & 0.0382 & 0.0367 & 0.0343 & 0.0348 & \\
\hline $120 \mathrm{~min}$ & 0.0360 & 0.0698 & 0.0474 & 0.0000 & 0.0356 & 0.0008 & 0.0382 & 0.0390 & 0.0387 & 0.0365 & 0.0344 & 0.0348 & \\
\hline $180 \mathrm{~min}$ & 0.0360 & 0.0387 & 0.0578 & 0.0000 & 0.0355 & 0.0008 & 0.0382 & 0.0391 & 0.0389 & 0.0363 & 0.0342 & 0.0346 & \\
\hline $240 \mathrm{~min}$ & 0.0360 & 0.0219 & 0.0636 & 0.0000 & 0.0355 & 0.0010 & 0.0382 & 0.0392 & 0.0391 & 0.0363 & 0.0344 & 0.0346 & \\
\hline $1155 \mathrm{~min}$ & 0.0360 & 0.0008 & 0.0714 & 0.0000 & 0.0342 & 0.0022 & 0.0381 & 0.0392 & 0.0391 & 0.0355 & 0.0329 & 0.0333 & \\
\hline
\end{tabular}

${ }^{a}$ Mesitylene (18 $\left.\mu \mathrm{mol}\right)$ was used as internal standard (IS).

${ }^{b}$ The integral was corrected for the overlapping carbon-13 NMR satellite of the acetate singlet and therefore slightly deviates from the integrals shown in the NMR spectra.

${ }^{c}$ Center of the respective integral. The range $\mathrm{x}-\mathrm{y}$ indicates the center of the integral in the first $(0 \mathrm{~min})$ and last $(1155 \mathrm{~min})$ NMR spectrum in case the signal shifted.

$d$ Width of the respective integral. The range $x$ - y indicates the width of the integral in the first $(0 \mathrm{~min})$ and last (1155 min) NMR spectrum. Different widths were chosen for some signals to avoid unnecessary overlapping.

${ }^{e}$ The integral could not be determined due to the overlapping $\alpha-\mathrm{CH}_{2}$ group of $\mathrm{NBu}_{4}{ }^{+}$. With progressive pre-catalyst activation, the signal of the latter group shifted. 


\subsection{2 ${ }^{13} \mathrm{C}$ NMR Spectra of Reaction Monitoring with Internal Standard}

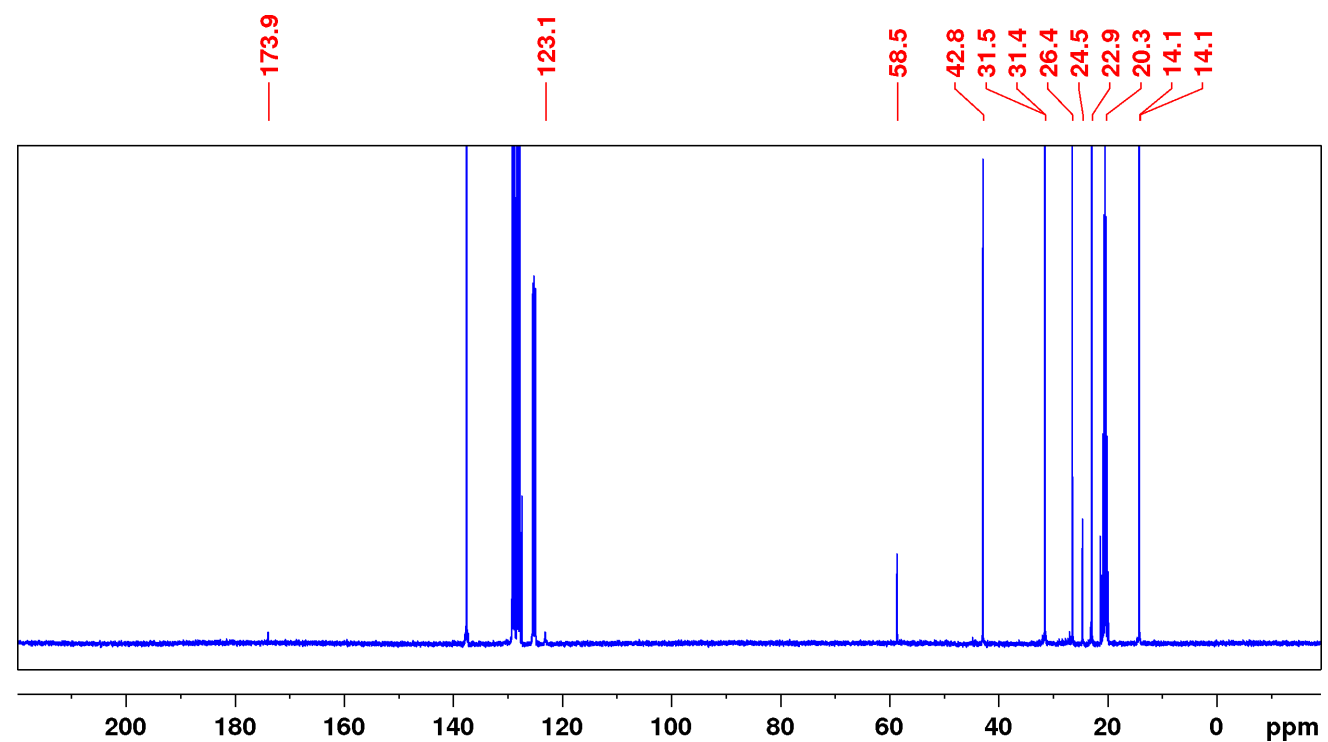

Figure S22: ${ }^{13} \mathrm{C}\left\{{ }^{1} \mathrm{H}\right\}$ NMR (Toluene- $\mathrm{d}_{8}, 101 \mathrm{MHz}, 298 \mathrm{~K}$ ) spectrum of a reaction mixture in a J. Young NMR tube after 0 min at $80^{\circ} \mathrm{C}$. Mesitylene $(18 \mu \mathrm{mol})$ was used as internal standard.

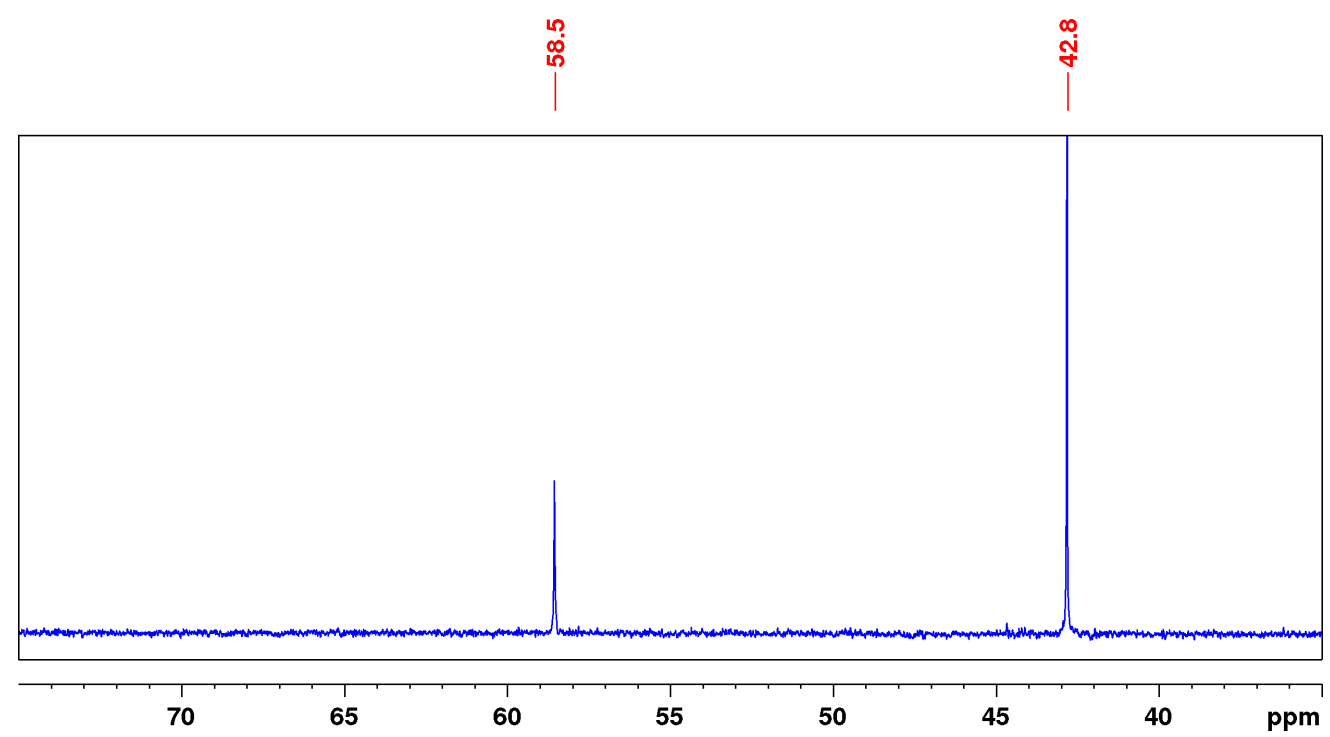

Figure S23: ${ }^{13} \mathrm{C}\left\{{ }^{1} \mathrm{H}\right\}$ NMR (Toluene- $\mathrm{d}_{8}, 101 \mathrm{MHz}, 298 \mathrm{~K}$ ) spectrum between 35 and $75 \mathrm{ppm}$ of a reaction mixture in a J. Young NMR tube after $0 \mathrm{~min}$ at $80^{\circ} \mathrm{C}$. Mesitylene $(18 \mu \mathrm{mol})$ was used as internal standard. 


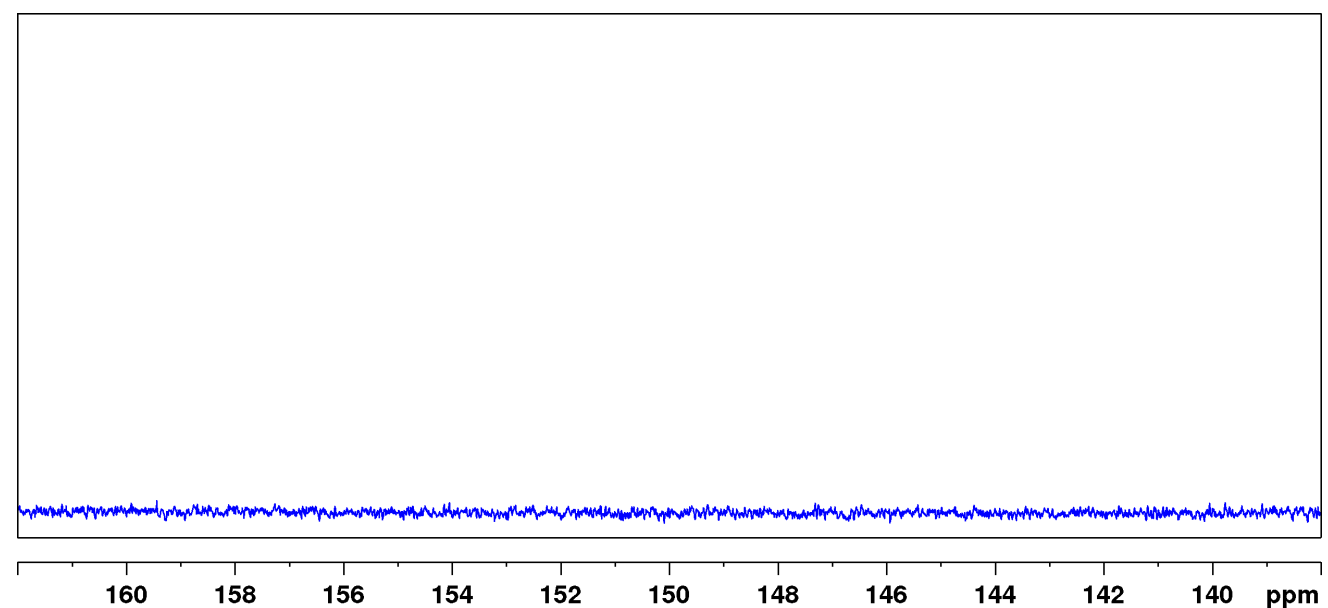

Figure S24: ${ }^{13} \mathrm{C}\left\{{ }^{1} \mathrm{H}\right\}$ NMR (Toluene- $\mathrm{d}_{8}, 101 \mathrm{MHz}, 298 \mathrm{~K}$ ) spectrum between 138 and $162 \mathrm{ppm}$ of a reaction mixture in a J. Young NMR tube after $0 \mathrm{~min}$ at $80^{\circ} \mathrm{C}$. Mesitylene $(18 \mu \mathrm{mol})$ was used as internal standard. 


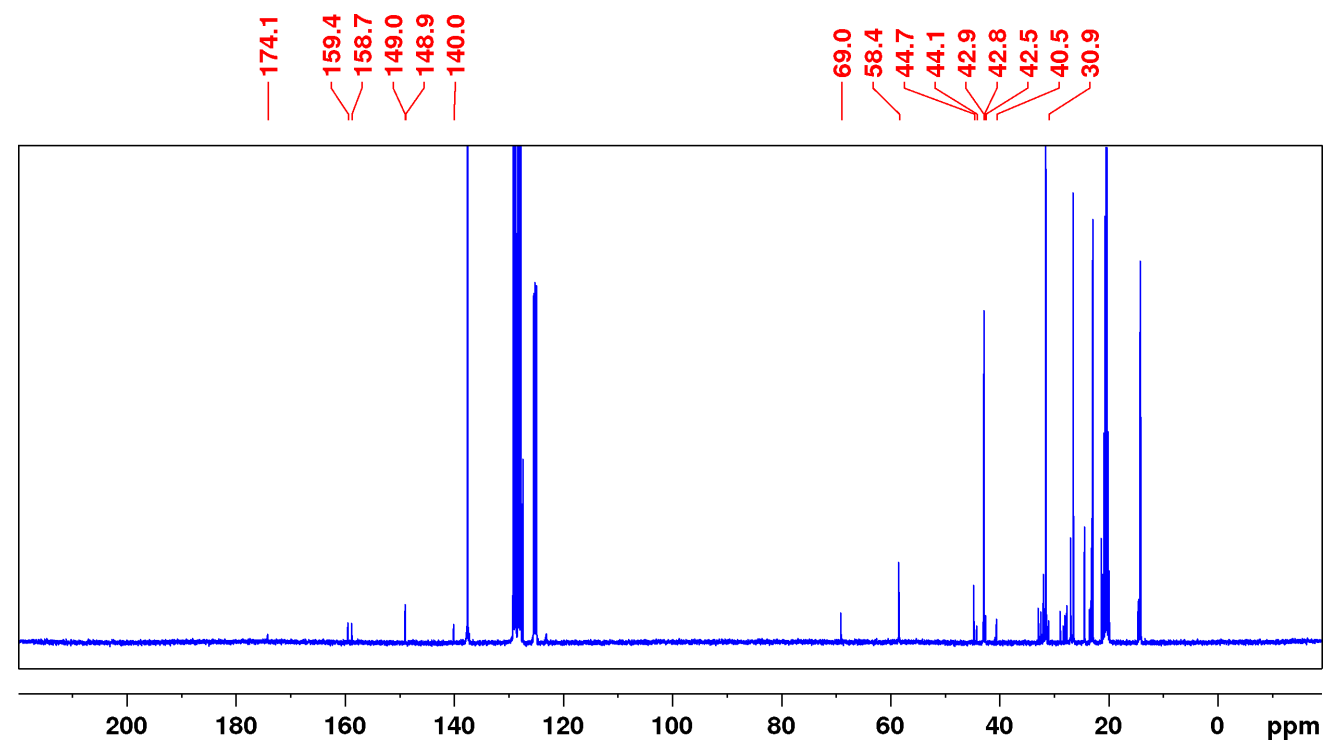

Figure S25: ${ }^{13} \mathrm{C}\left\{{ }^{1} \mathrm{H}\right\}$ NMR (Toluene- $\mathrm{d}_{8}, 101 \mathrm{MHz}, 298 \mathrm{~K}$ ) spectrum of a reaction mixture in a J. Young NMR tube after $5 \mathrm{~min}$ at $80^{\circ} \mathrm{C}$. Mesitylene $(18 \mu \mathrm{mol})$ was used as internal standard.

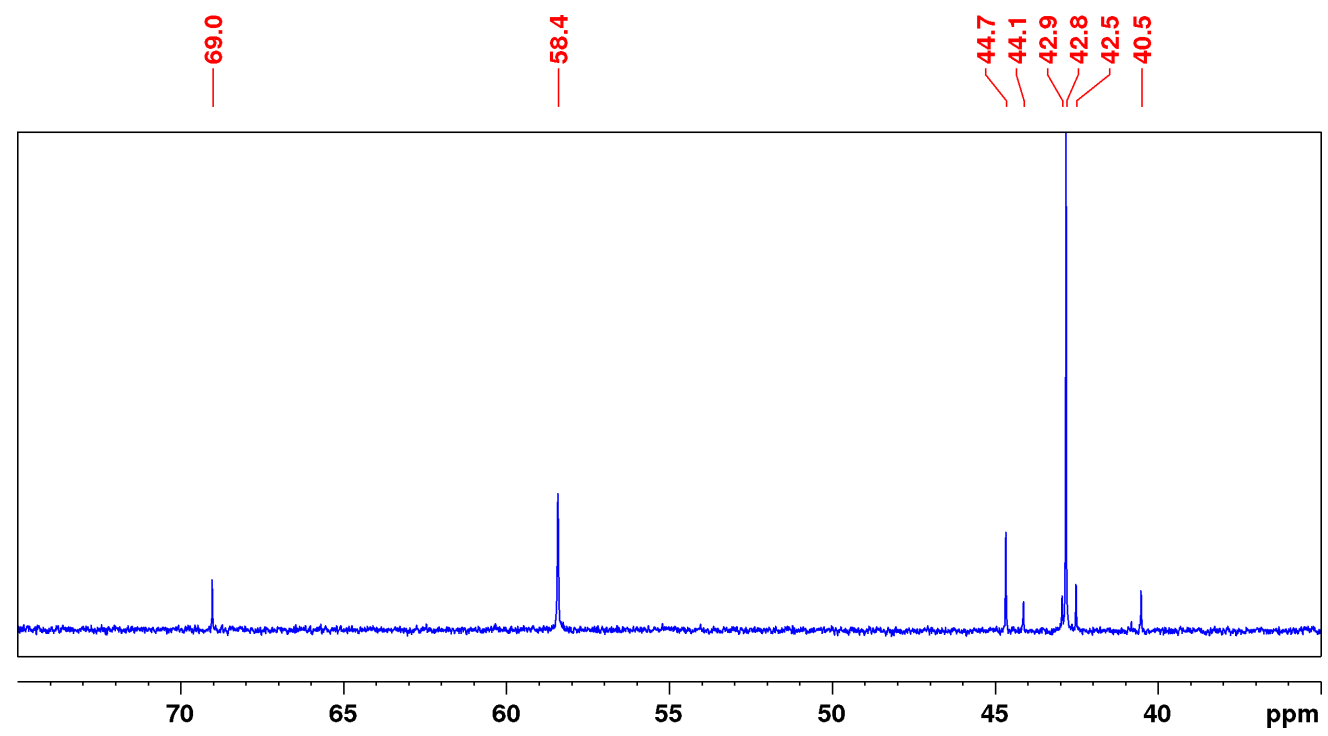

Figure S26: ${ }^{13} \mathrm{C}\left\{{ }^{1} \mathrm{H}\right\}$ NMR (Toluene- $\mathrm{d}_{8}, 101 \mathrm{MHz}, 298 \mathrm{~K}$ ) spectrum between 35 and $75 \mathrm{ppm}$ of a reaction mixture in a J. Young NMR tube after $5 \mathrm{~min}$ at $80^{\circ} \mathrm{C}$. Mesitylene $(18 \mu \mathrm{mol})$ was used as internal standard. 


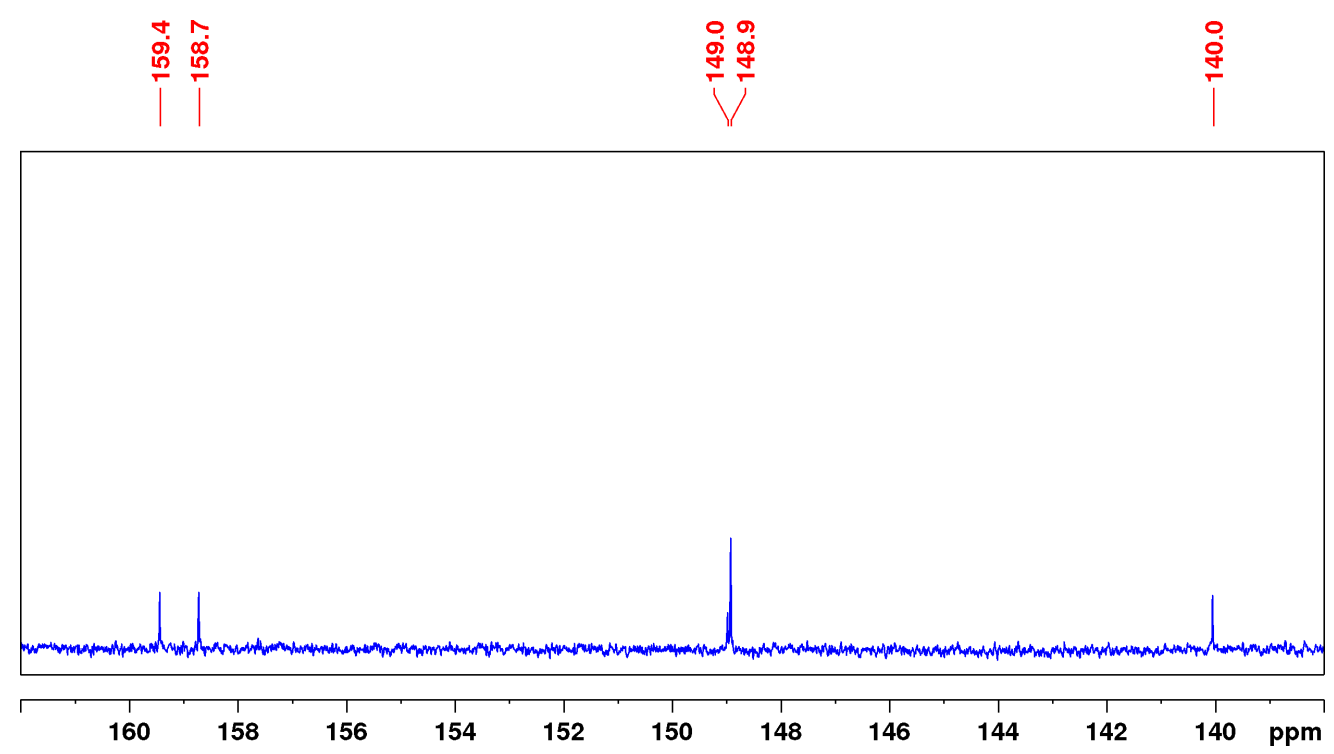

Figure S27: ${ }^{13} \mathrm{C}\left\{{ }^{1} \mathrm{H}\right\}$ NMR (Toluene- $\mathrm{d}_{8}, 101 \mathrm{MHz}, 298 \mathrm{~K}$ ) spectrum between 138 and $162 \mathrm{ppm}$ of a reaction mixture in a J. Young NMR tube after $5 \mathrm{~min}$ at $80^{\circ} \mathrm{C}$. Mesitylene $(18 \mu \mathrm{mol})$ was used as internal standard. 


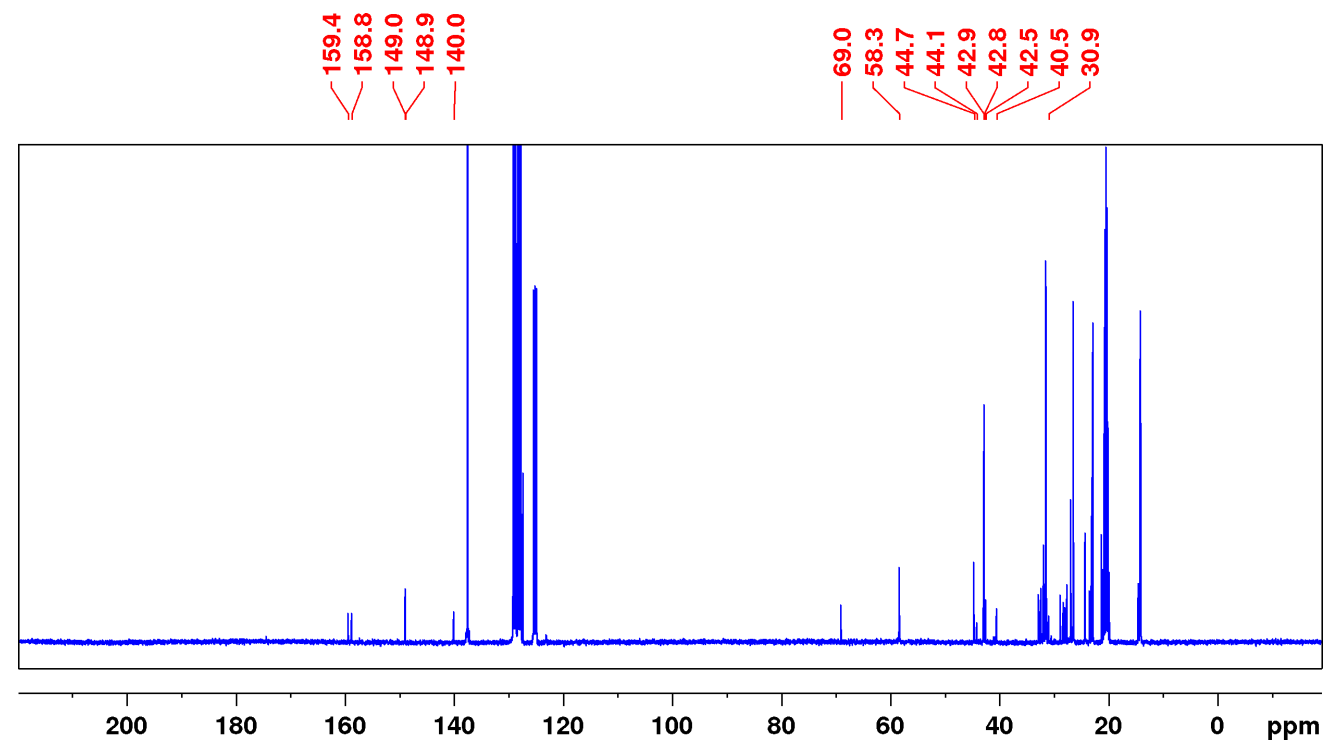

Figure S28: ${ }^{13} \mathrm{C}\left\{{ }^{1} \mathrm{H}\right\}$ NMR (Toluene- $\mathrm{d}_{8}, 101 \mathrm{MHz}, 298 \mathrm{~K}$ ) spectrum of a reaction mixture in a J. Young NMR tube after $15 \mathrm{~min}$ at $80^{\circ} \mathrm{C}$. Mesitylene $(18 \mu \mathrm{mol})$ was used as internal standard.

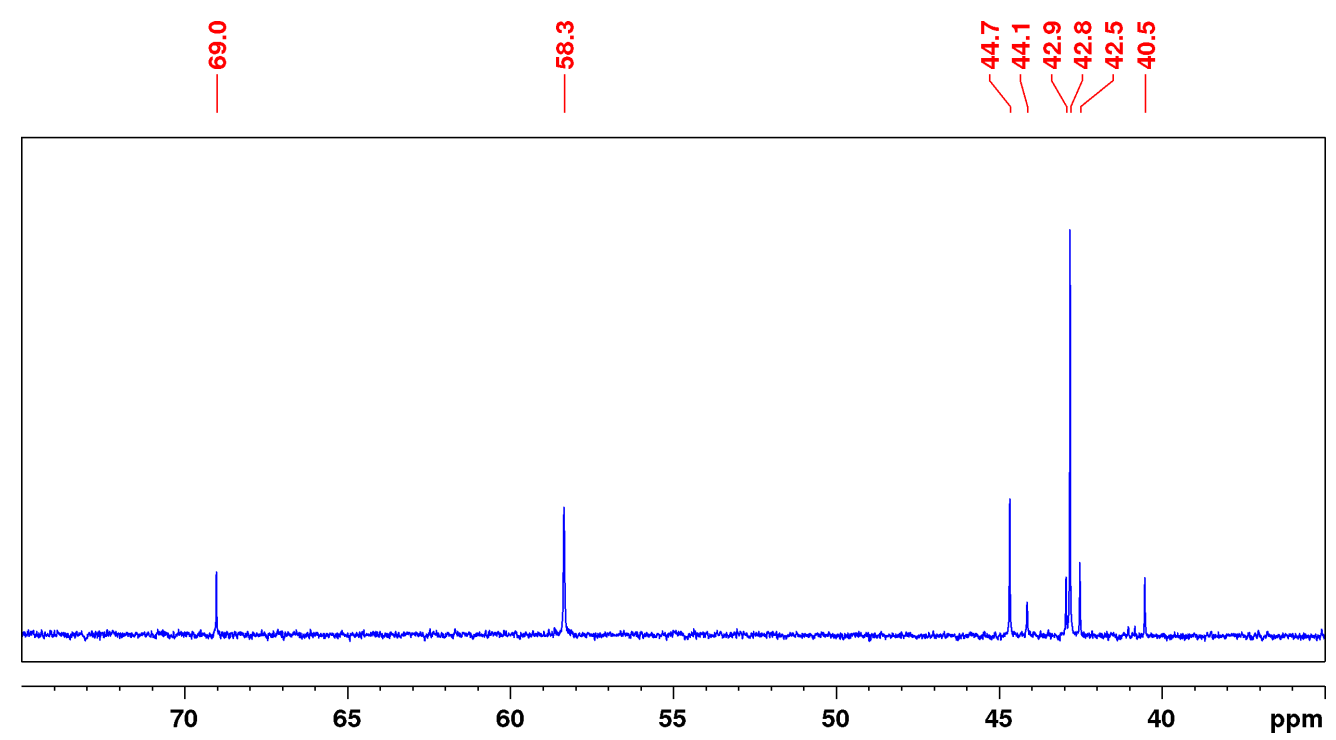

Figure S29: ${ }^{13} \mathrm{C}\left\{{ }^{1} \mathrm{H}\right\} \mathrm{NMR}$ (Toluene- $\mathrm{d}_{8}, 101 \mathrm{MHz}, 298 \mathrm{~K}$ ) spectrum between 35 and $75 \mathrm{ppm}$ of a reaction mixture in a J. Young NMR tube after $15 \mathrm{~min}$ at $80^{\circ} \mathrm{C}$. Mesitylene $(18 \mu \mathrm{mol})$ was used as internal standard. 


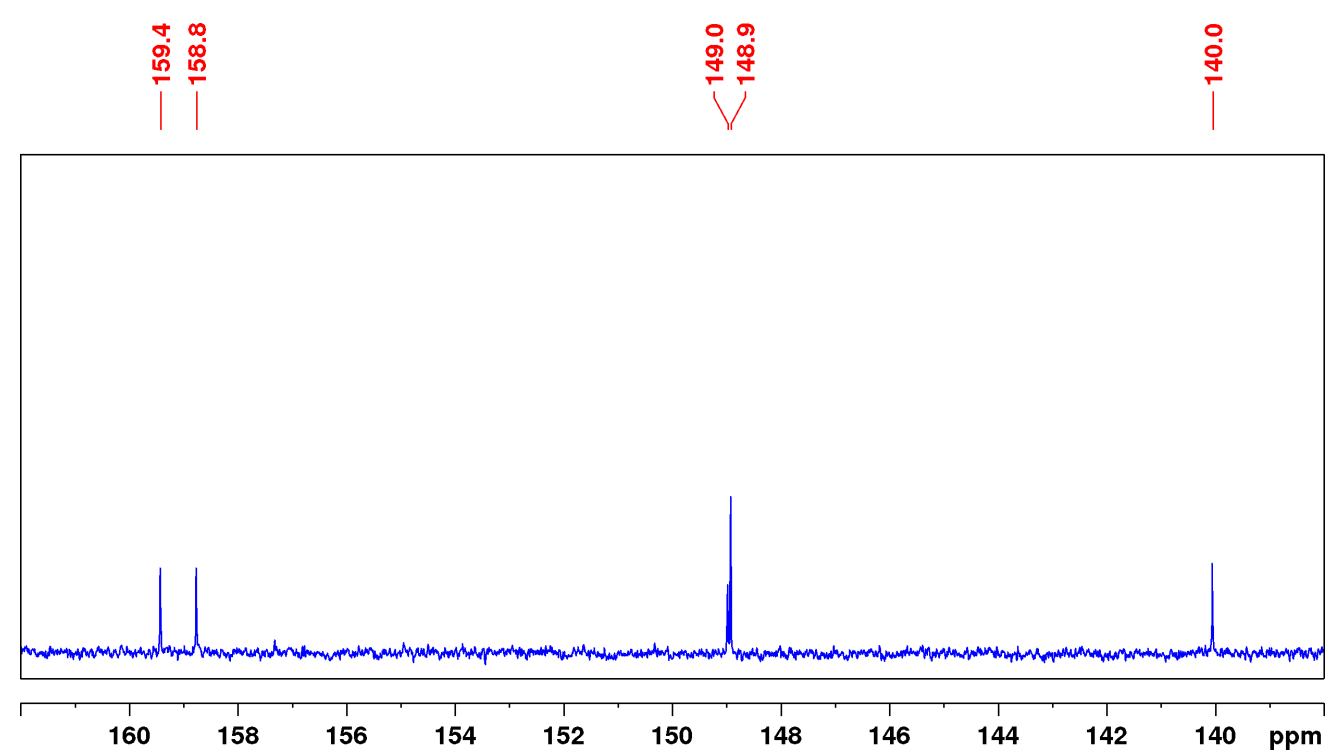

Figure S30: ${ }^{13} \mathrm{C}\left\{{ }^{1} \mathrm{H}\right\}$ NMR (Toluene- $\mathrm{d}_{8}, 101 \mathrm{MHz}, 298 \mathrm{~K}$ ) spectrum between 138 and $162 \mathrm{ppm}$ of a reaction mixture in a J. Young NMR tube after $15 \mathrm{~min}$ at $80^{\circ} \mathrm{C}$. Mesitylene $(18 \mu \mathrm{mol})$ was used as internal standard. 


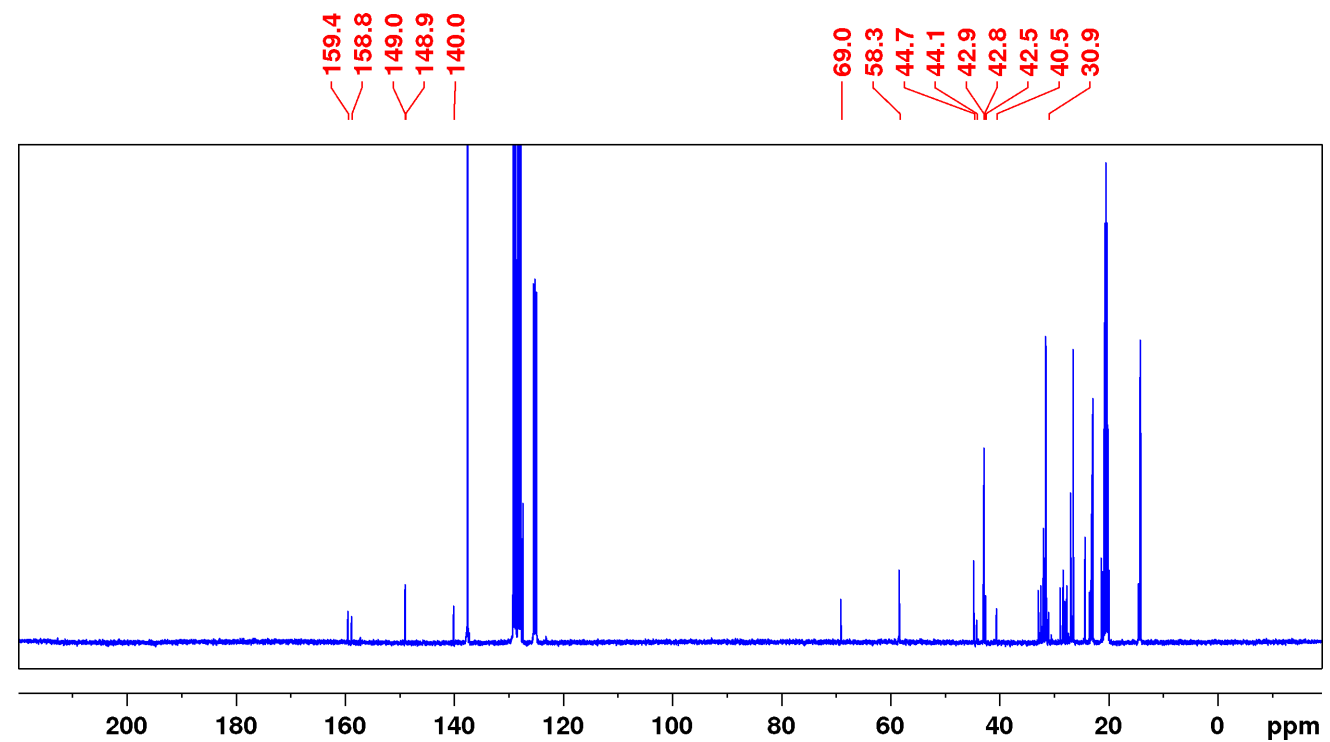

Figure S31: ${ }^{13} \mathrm{C}\left\{{ }^{1} \mathrm{H}\right\}$ NMR (Toluene- $\mathrm{d}_{8}, 101 \mathrm{MHz}, 298 \mathrm{~K}$ ) spectrum of a reaction mixture in a J. Young NMR tube after $30 \mathrm{~min}$ at $80^{\circ} \mathrm{C}$. Mesitylene $(18 \mu \mathrm{mol})$ was used as internal standard.

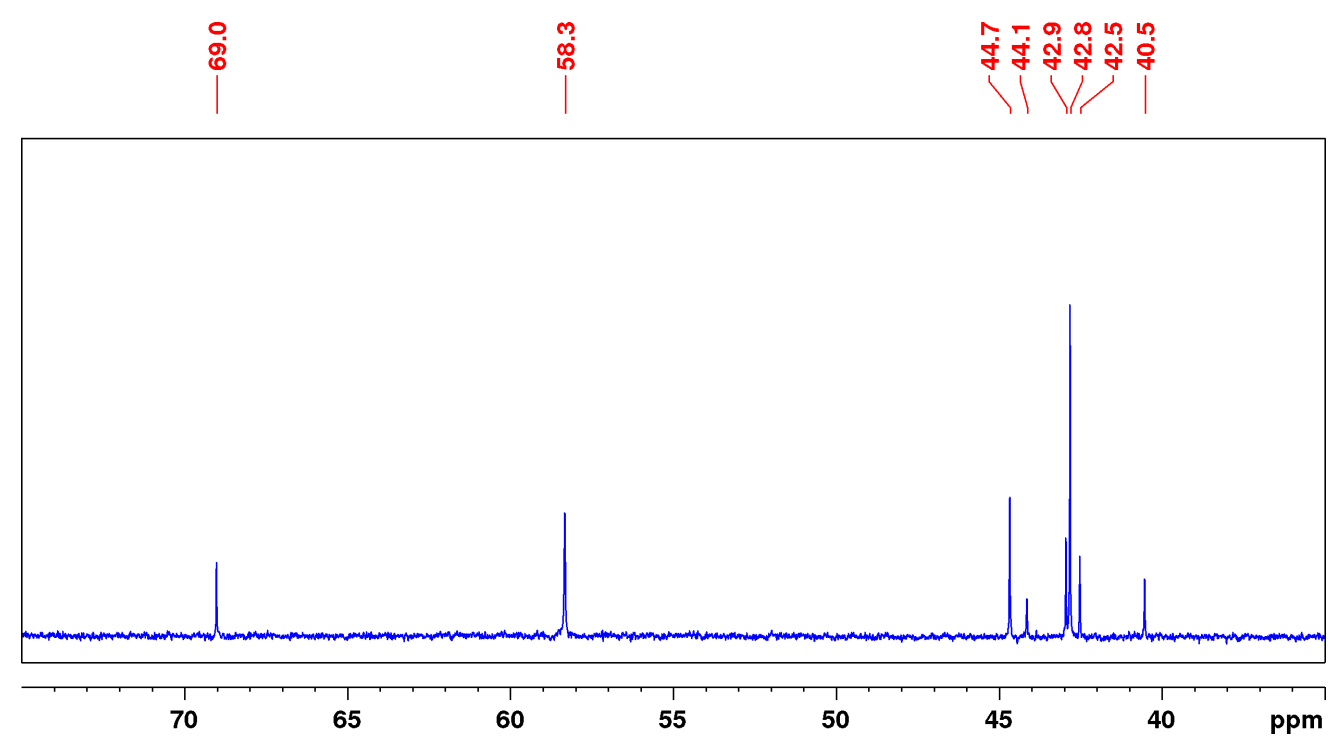

Figure S32: ${ }^{13} \mathrm{C}\left\{{ }^{1} \mathrm{H}\right\}$ NMR (Toluene-d $8,101 \mathrm{MHz}, 298 \mathrm{~K}$ ) spectrum between 35 and 75 ppm of a reaction mixture in a J. Young NMR tube after $30 \mathrm{~min}$ at $80^{\circ} \mathrm{C}$. Mesitylene $(18 \mu \mathrm{mol})$ was used as internal standard. 


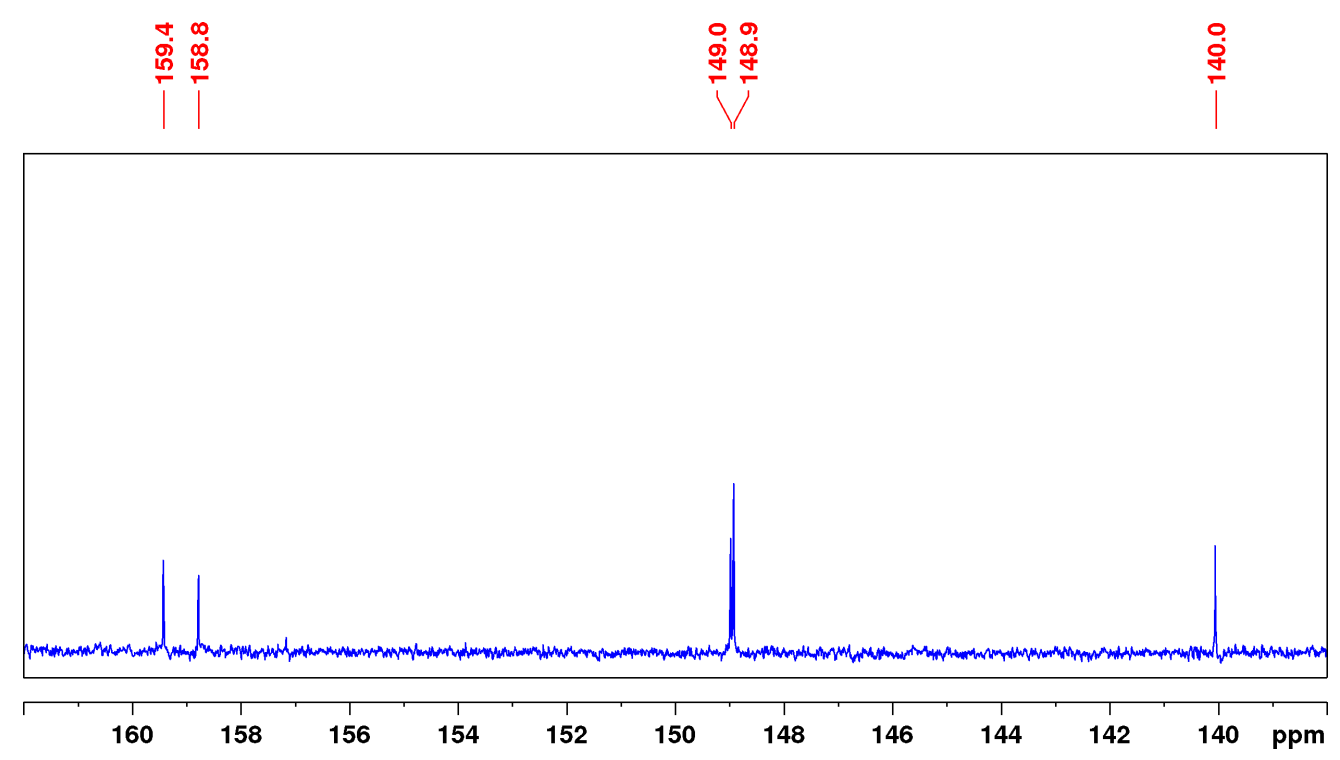

Figure S33: ${ }^{13} \mathrm{C}\left\{{ }^{1} \mathrm{H}\right\}$ NMR (Toluene- $\mathrm{d}_{8}, 101 \mathrm{MHz}, 298 \mathrm{~K}$ ) spectrum between 138 and $162 \mathrm{ppm}$ of a reaction mixture in a J. Young NMR tube after $30 \mathrm{~min}$ at $80^{\circ} \mathrm{C}$. Mesitylene $(18 \mu \mathrm{mol})$ was used as internal standard. 


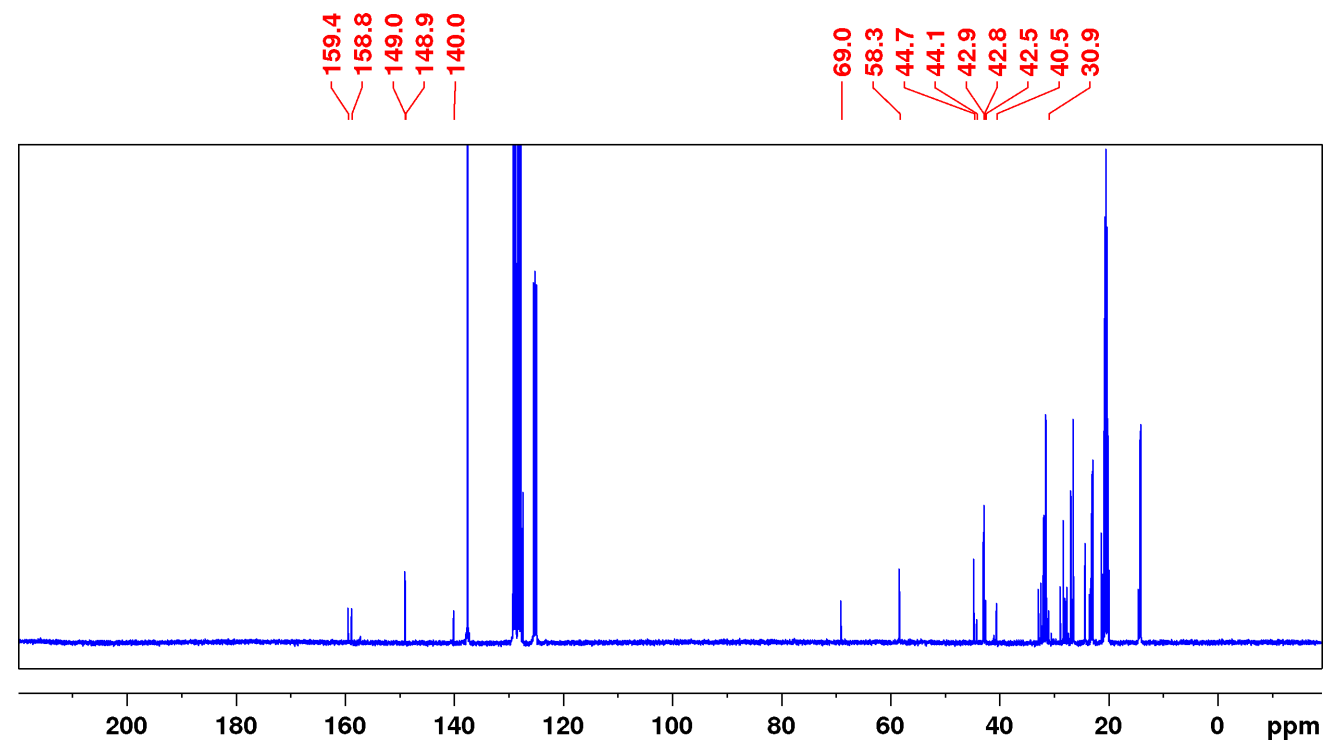

Figure S34: ${ }^{13} \mathrm{C}\left\{{ }^{1} \mathrm{H}\right\}$ NMR (Toluene- $\mathrm{d}_{8}, 101 \mathrm{MHz}, 298 \mathrm{~K}$ ) spectrum of a reaction mixture in a J. Young NMR tube after $60 \mathrm{~min}$ at $80^{\circ} \mathrm{C}$. Mesitylene $(18 \mu \mathrm{mol})$ was used as internal standard.

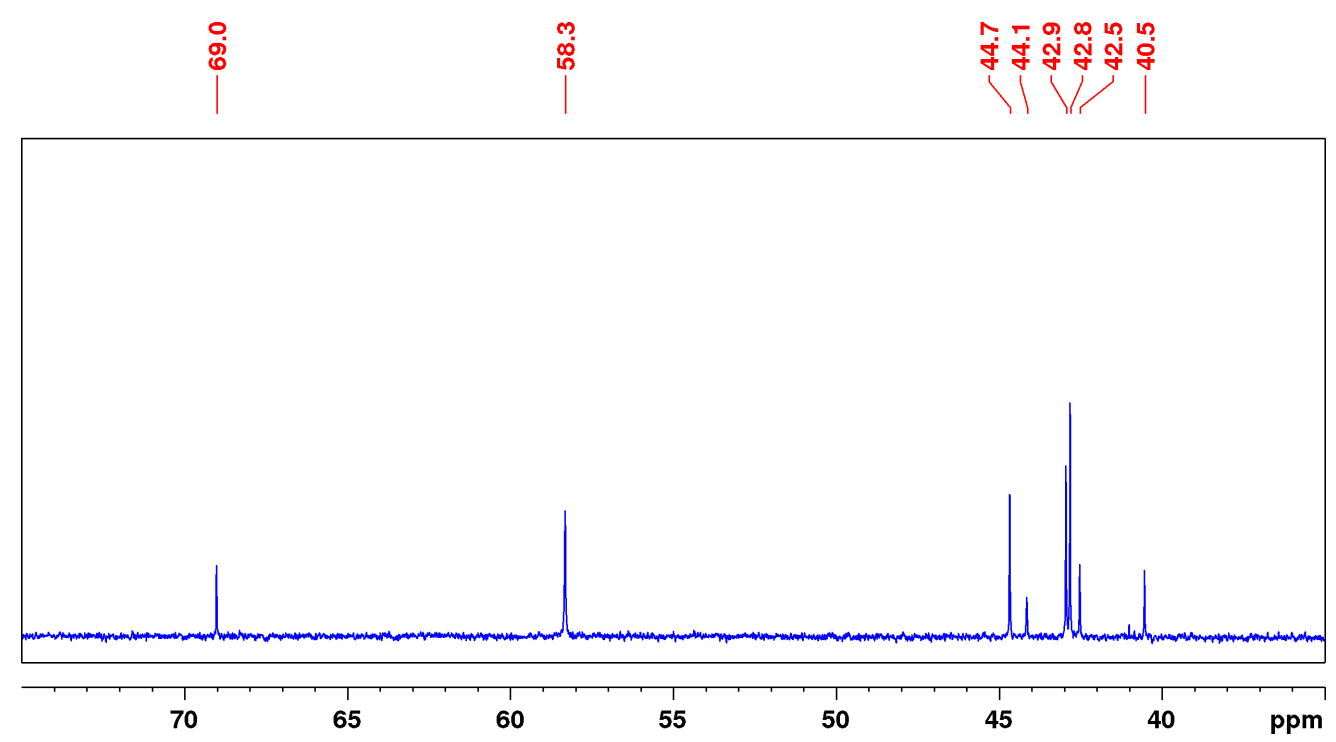

Figure S35: ${ }^{13} \mathrm{C}\left\{{ }^{1} \mathrm{H}\right\}$ NMR (Toluene-d $8,101 \mathrm{MHz}, 298 \mathrm{~K}$ ) spectrum between 35 and $75 \mathrm{ppm}$ of a reaction mixture in a J. Young NMR tube after $60 \mathrm{~min}$ at $80^{\circ} \mathrm{C}$. Mesitylene $(18 \mu \mathrm{mol})$ was used as internal standard. 


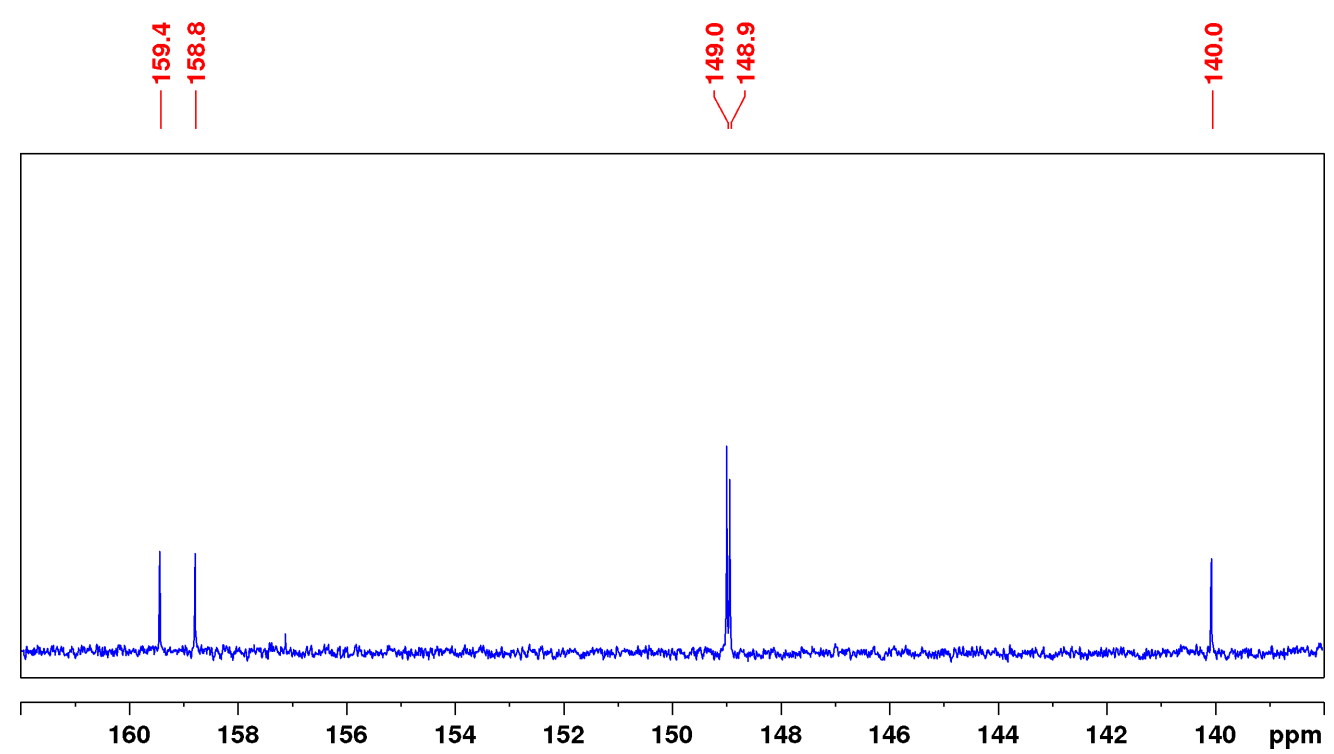

Figure S36: ${ }^{13} \mathrm{C}\left\{{ }^{1} \mathrm{H}\right\}$ NMR (Toluene- $\mathrm{d}_{8}, 101 \mathrm{MHz}, 298 \mathrm{~K}$ ) spectrum between 138 and $162 \mathrm{ppm}$ of a reaction mixture in a J. Young NMR tube after $60 \mathrm{~min}$ at $80^{\circ} \mathrm{C}$. Mesitylene $(18 \mu \mathrm{mol})$ was used as internal standard. 


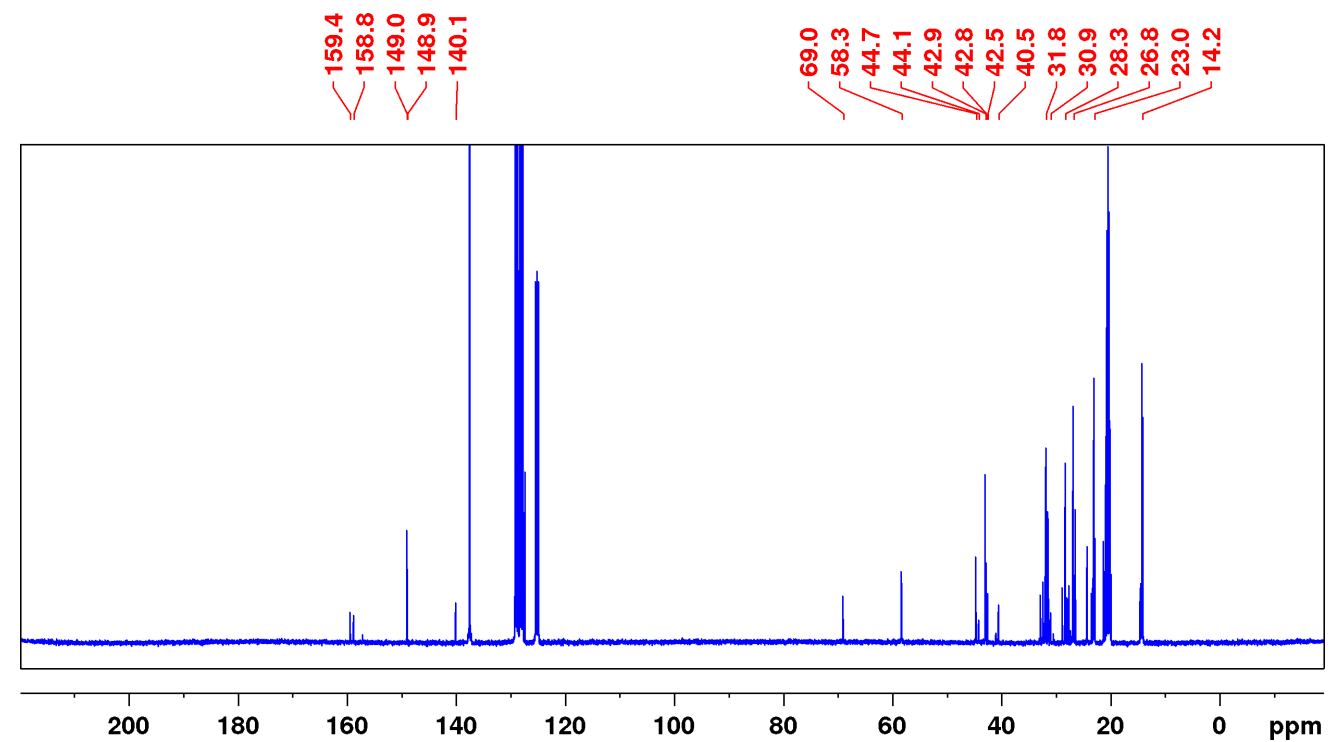

Figure S37: ${ }^{13} \mathrm{C}\left\{{ }^{1} \mathrm{H}\right\}$ NMR (Toluene- $\mathrm{d}_{8}, 101 \mathrm{MHz}, 298 \mathrm{~K}$ ) spectrum of a reaction mixture in a J. Young NMR tube after $120 \mathrm{~min}$ at $80^{\circ} \mathrm{C}$. Mesitylene $(18 \mu \mathrm{mol})$ was used as internal standard.

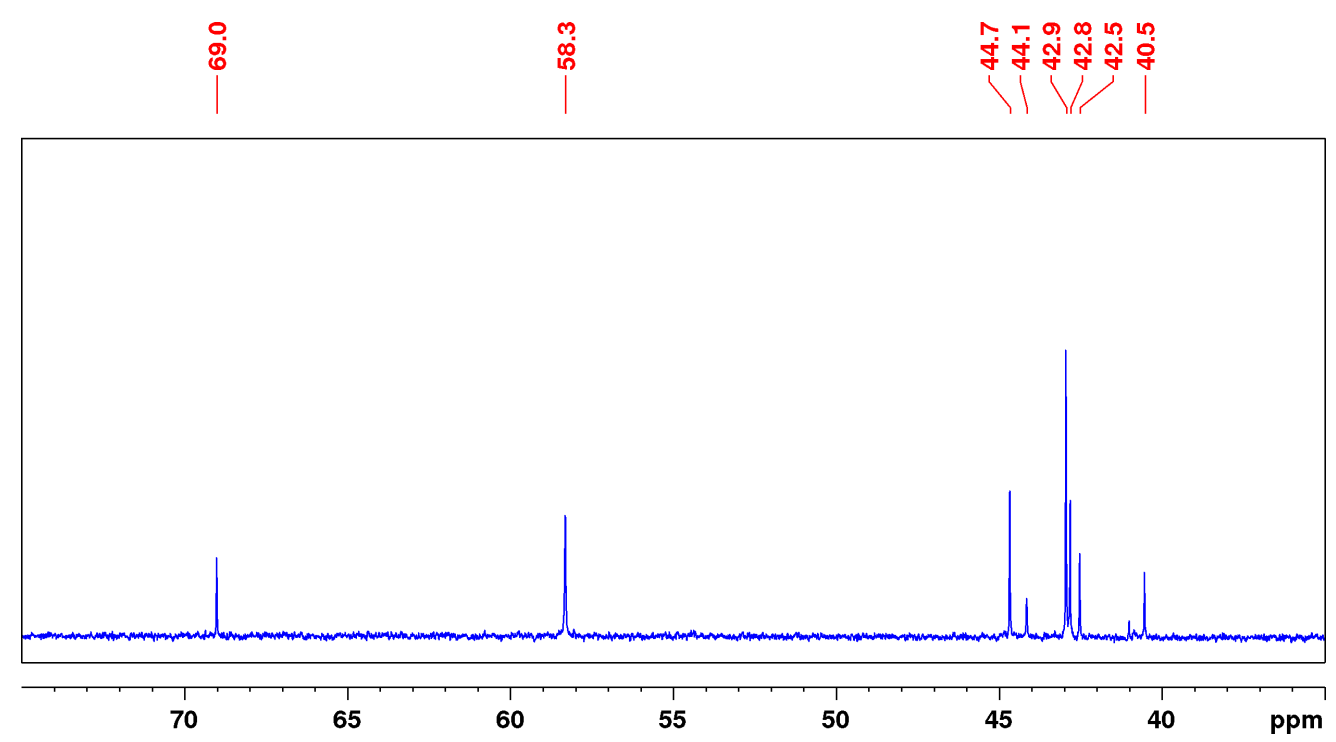

Figure S38: ${ }^{13} \mathrm{C}\left\{{ }^{1} \mathrm{H}\right\}$ NMR (Toluene-d $8,101 \mathrm{MHz}, 298 \mathrm{~K}$ ) spectrum between 35 and $75 \mathrm{ppm}$ of a reaction mixture in a J. Young NMR tube after $120 \mathrm{~min}$ at $80^{\circ} \mathrm{C}$. Mesitylene $(18 \mu \mathrm{mol})$ was used as internal standard. 


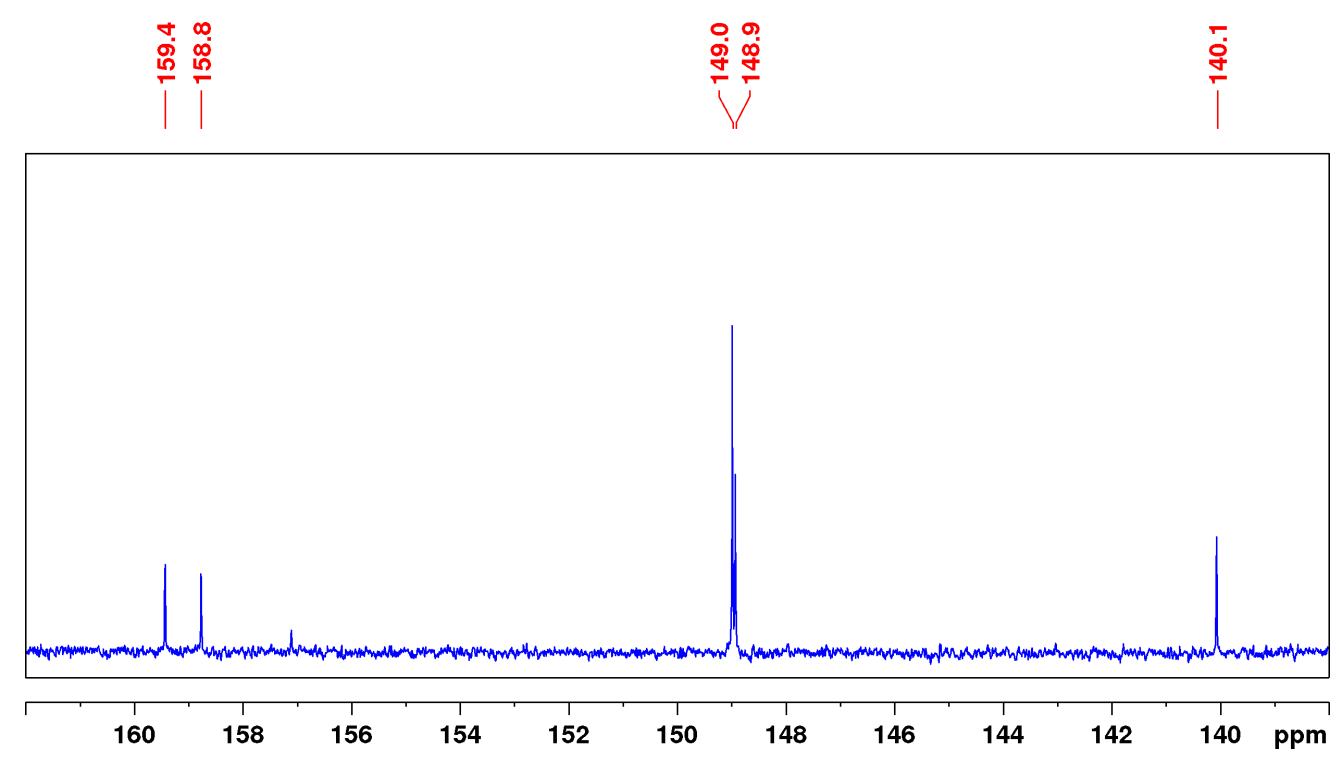

Figure S39: ${ }^{13} \mathrm{C}\left\{{ }^{1} \mathrm{H}\right\}$ NMR (Toluene- $\mathrm{d}_{8}, 101 \mathrm{MHz}, 298 \mathrm{~K}$ ) spectrum between 138 and $162 \mathrm{ppm}$ of a reaction mixture in a J. Young NMR tube after $120 \mathrm{~min}$ at $80^{\circ} \mathrm{C}$. Mesitylene $(18 \mu \mathrm{mol})$ was used as internal standard. 


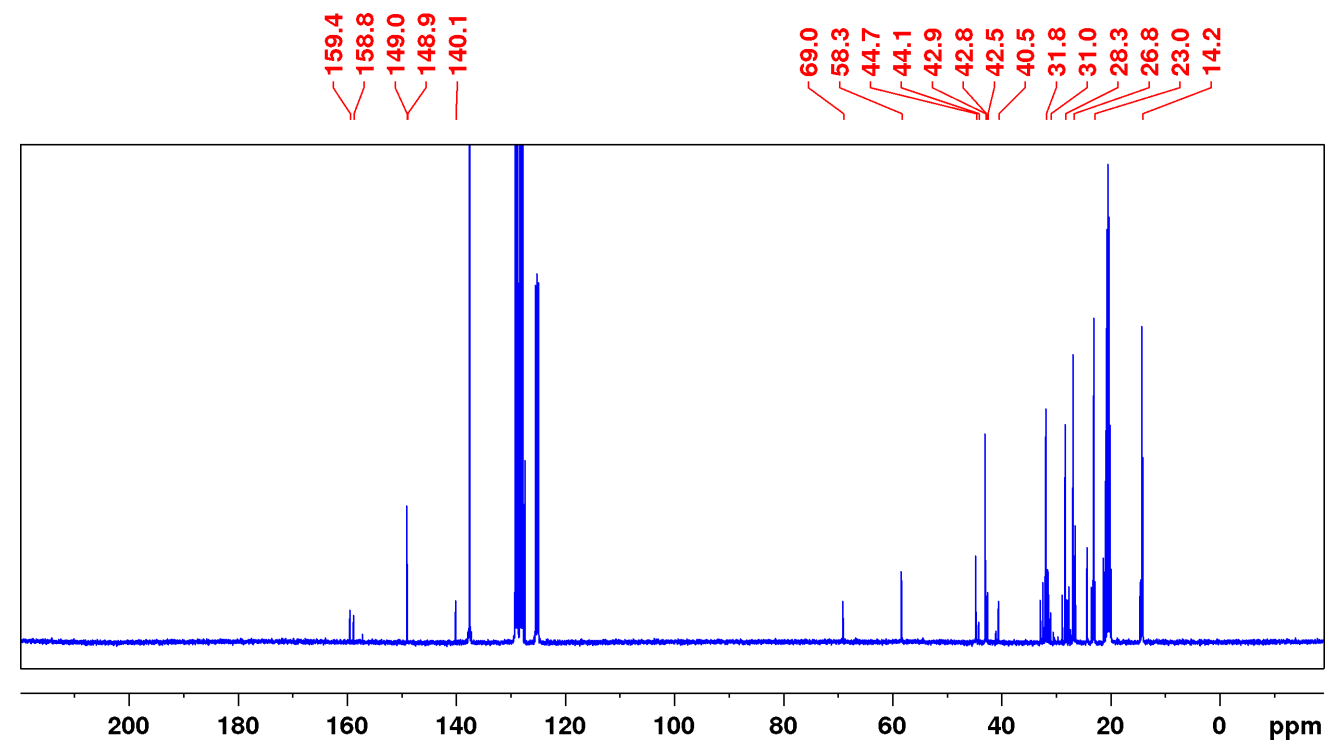

Figure S40: ${ }^{13} \mathrm{C}\left\{{ }^{1} \mathrm{H}\right\}$ NMR (Toluene- $\mathrm{d}_{8}, 101 \mathrm{MHz}, 298 \mathrm{~K}$ ) spectrum of a reaction mixture in a J. Young NMR tube after $180 \mathrm{~min}$ at $80^{\circ} \mathrm{C}$. Mesitylene $(18 \mu \mathrm{mol})$ was used as internal standard.

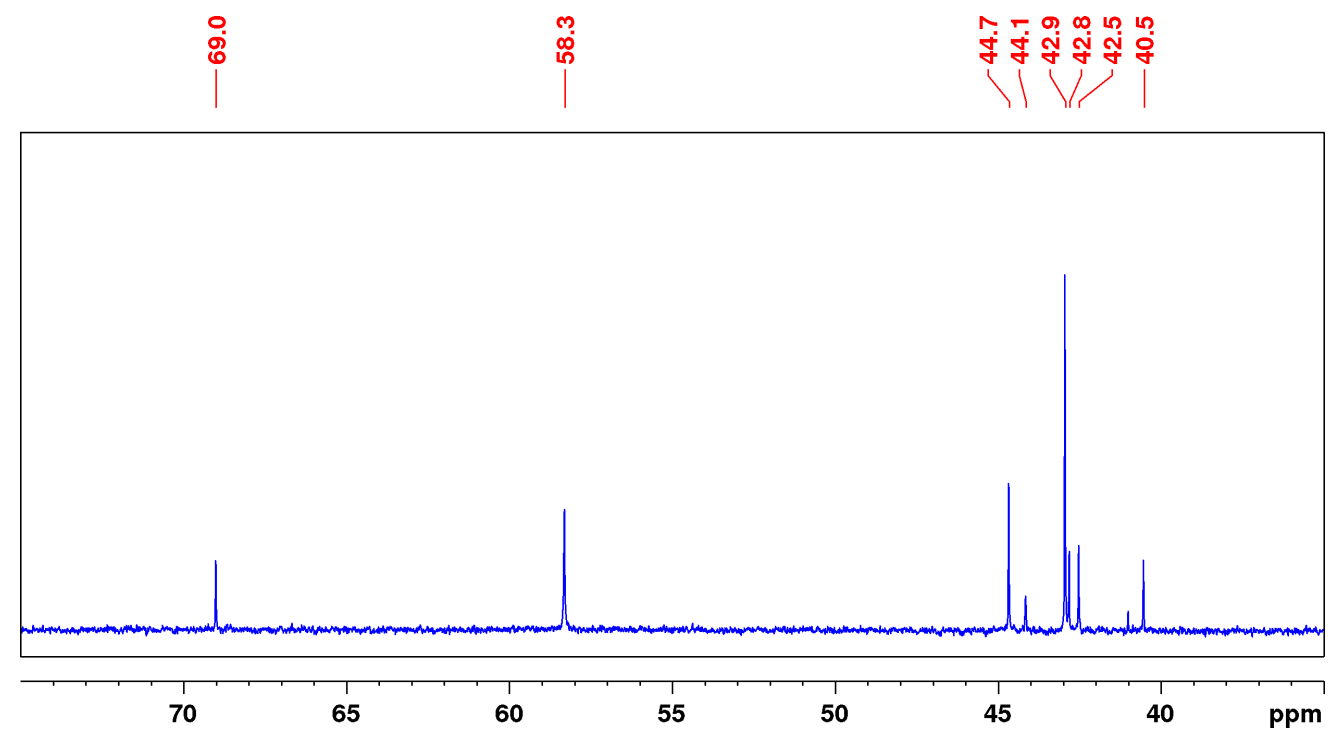

Figure S41: ${ }^{13} \mathrm{C}\left\{{ }^{1} \mathrm{H}\right\}$ NMR (Toluene- $\mathrm{d}_{8}, 101 \mathrm{MHz}, 298 \mathrm{~K}$ ) spectrum between 35 and $75 \mathrm{ppm}$ of a reaction mixture in a J. Young NMR tube after $180 \mathrm{~min}$ at $80^{\circ} \mathrm{C}$. Mesitylene $(18 \mu \mathrm{mol})$ was used as internal standard. 


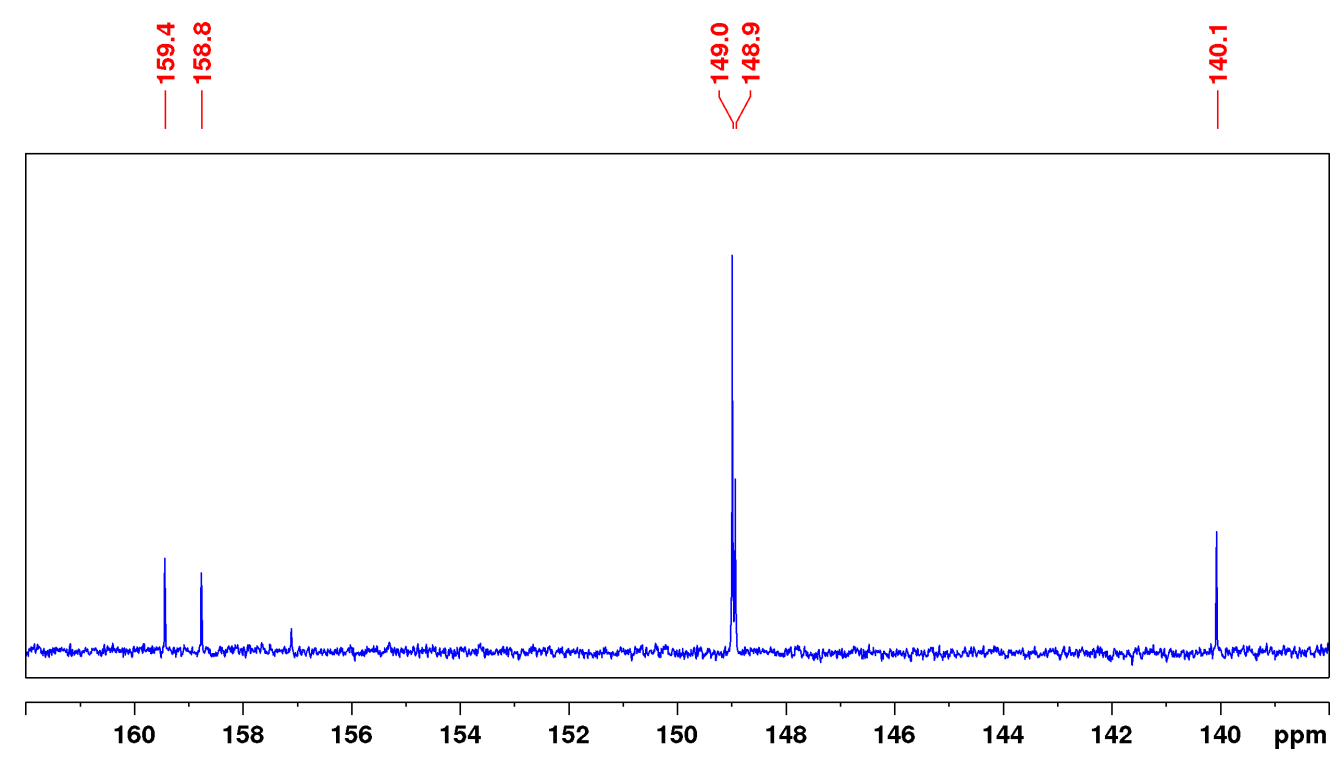

Figure S42: ${ }^{13} \mathrm{C}\left\{{ }^{1} \mathrm{H}\right\} \mathrm{NMR}$ (Toluene- $\mathrm{d}_{8}, 101 \mathrm{MHz}, 298 \mathrm{~K}$ ) spectrum between 138 and $162 \mathrm{ppm}$ of a reaction mixture in a J. Young NMR tube after $180 \mathrm{~min}$ at $80^{\circ} \mathrm{C}$. Mesitylene $(18 \mu \mathrm{mol})$ was used as internal standard. 


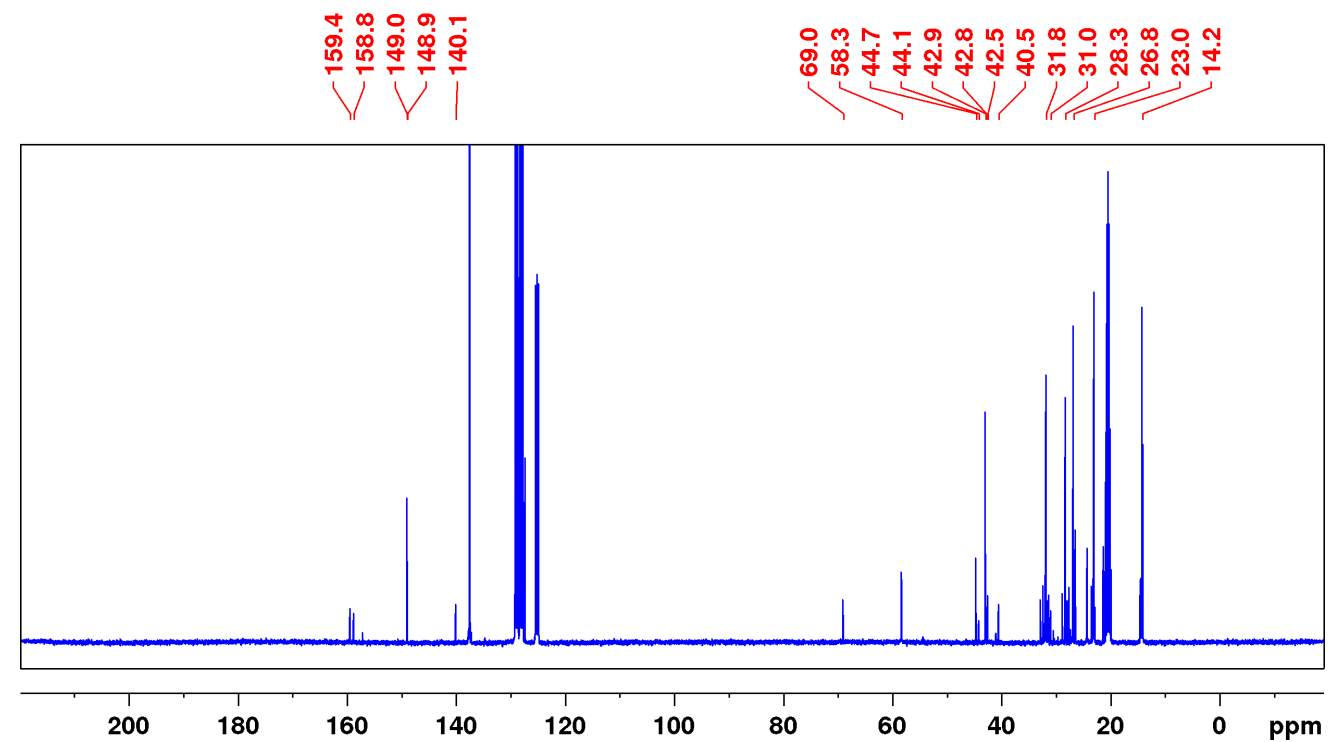

Figure S43: ${ }^{13} \mathrm{C}\left\{{ }^{1} \mathrm{H}\right\}$ NMR (Toluene- $\mathrm{d}_{8}, 101 \mathrm{MHz}, 298 \mathrm{~K}$ ) spectrum of a reaction mixture in a J. Young NMR tube after $240 \mathrm{~min}$ at $80^{\circ} \mathrm{C}$. Mesitylene $(18 \mu \mathrm{mol})$ was used as internal standard.

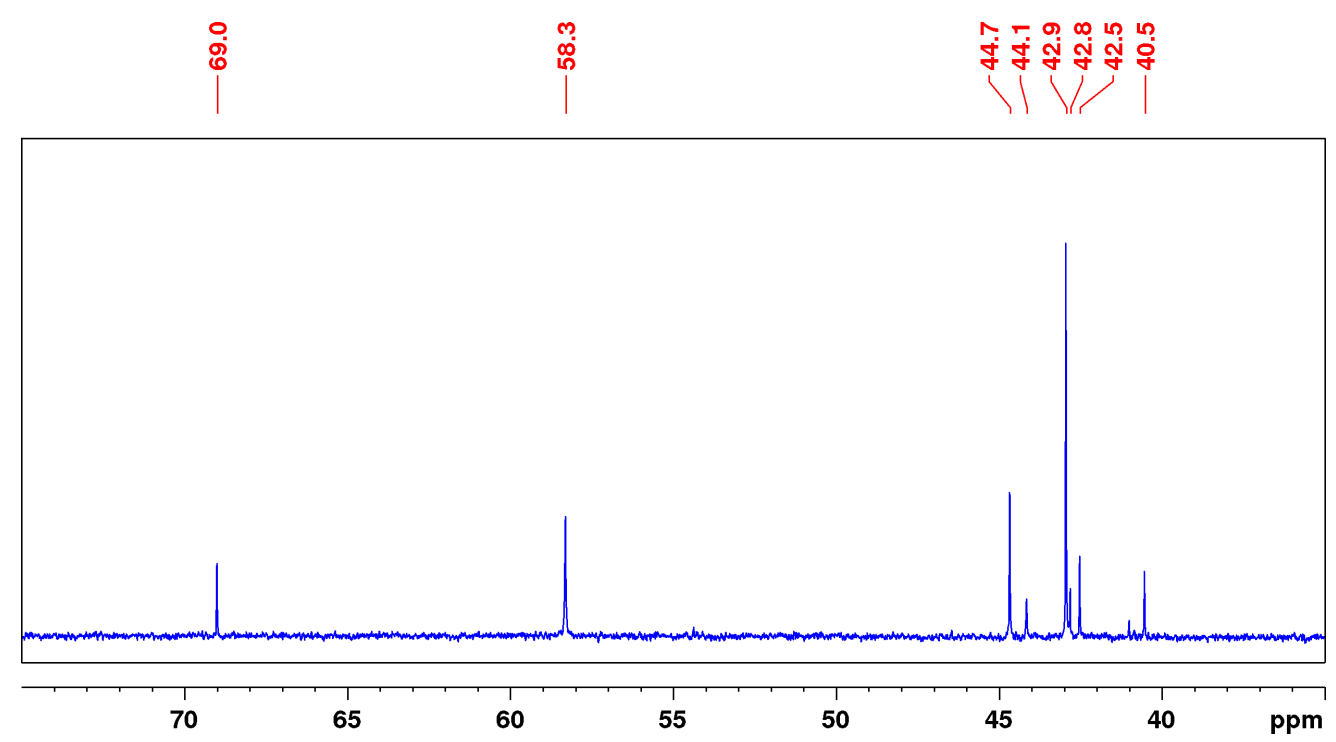

Figure S44: ${ }^{13} \mathrm{C}\left\{{ }^{1} \mathrm{H}\right\}$ NMR (Toluene-d $8,101 \mathrm{MHz}, 298 \mathrm{~K}$ ) spectrum between 35 and $75 \mathrm{ppm}$ of a reaction mixture in a J. Young NMR tube after $240 \mathrm{~min}$ at $80^{\circ} \mathrm{C}$. Mesitylene $(18 \mu \mathrm{mol})$ was used as internal standard. 


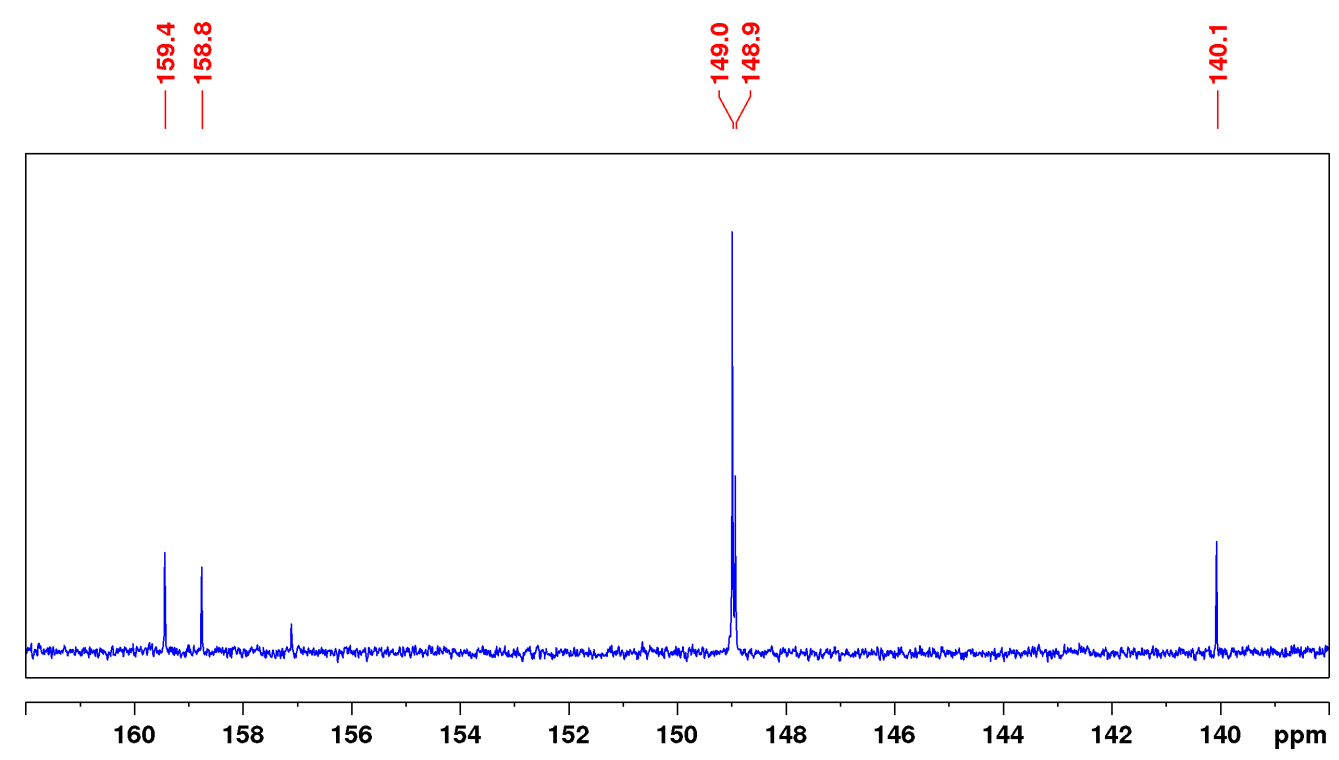

Figure S45: ${ }^{13} \mathrm{C}\left\{{ }^{1} \mathrm{H}\right\}$ NMR (Toluene- $\mathrm{d}_{8}, 101 \mathrm{MHz}, 298 \mathrm{~K}$ ) spectrum between 138 and $162 \mathrm{ppm}$ of a reaction mixture in a J. Young NMR tube after $240 \mathrm{~min}$ at $80^{\circ} \mathrm{C}$. Mesitylene $(18 \mu \mathrm{mol})$ was used as internal standard. 


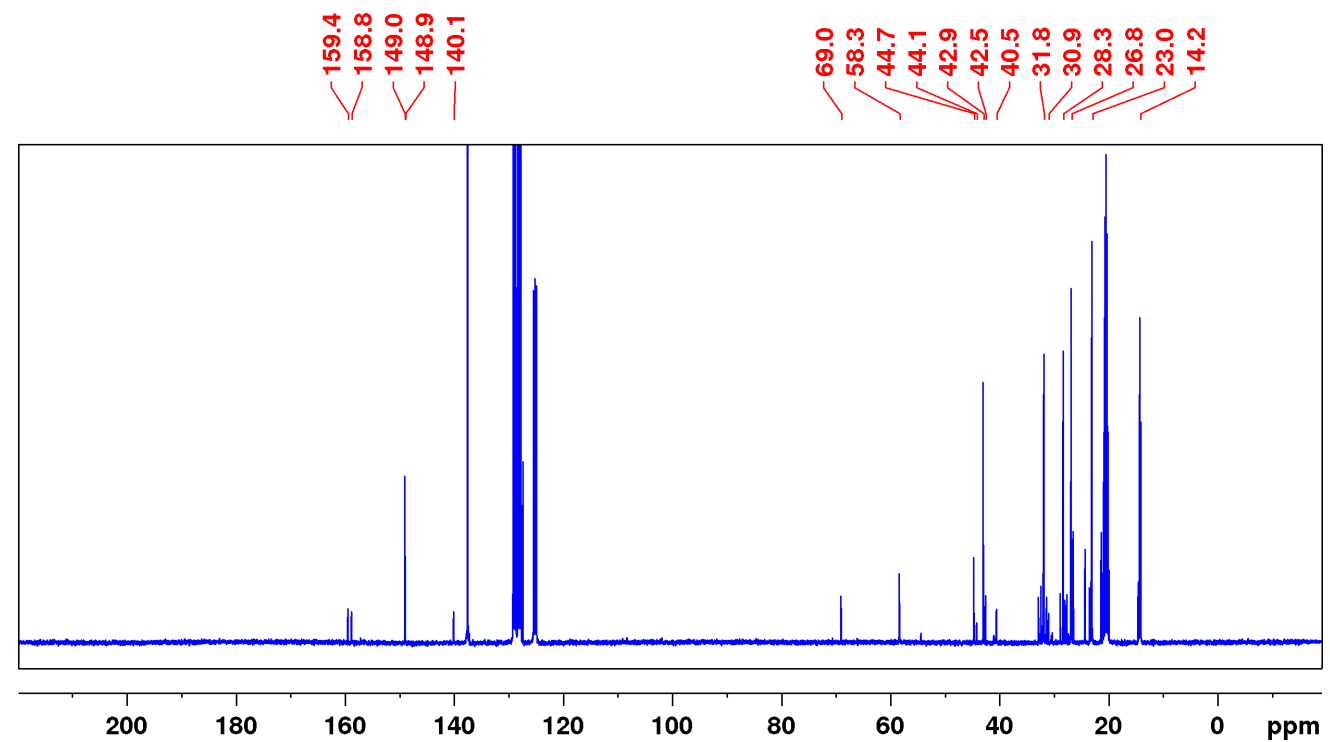

Figure S46: ${ }^{13} \mathrm{C}\left\{{ }^{1} \mathrm{H}\right\}$ NMR (Toluene- $\mathrm{d}_{8}, 101 \mathrm{MHz}, 298 \mathrm{~K}$ ) spectrum of a reaction mixture in a J. Young NMR tube after $1155 \mathrm{~min}$ at $80^{\circ} \mathrm{C}$. Mesitylene $(18 \mu \mathrm{mol})$ was used as internal standard.

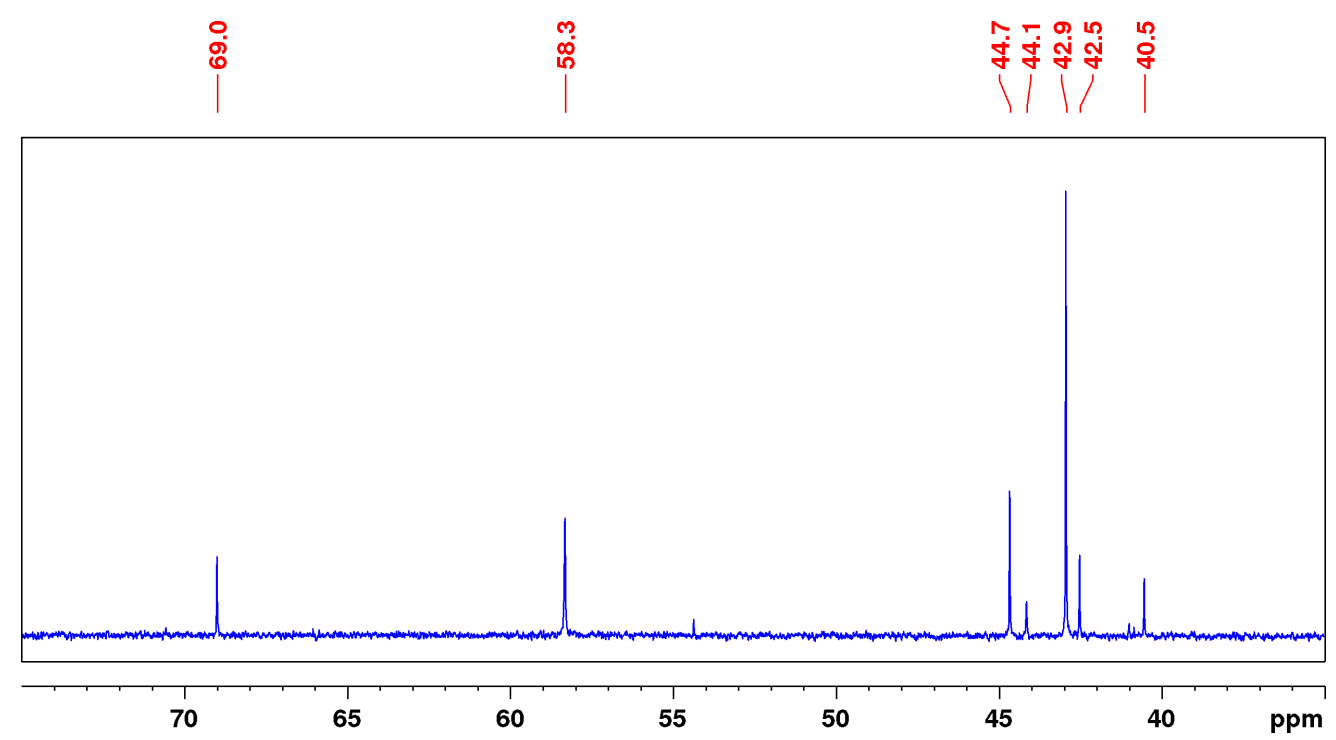

Figure S47: ${ }^{13} \mathrm{C}\left\{{ }^{1} \mathrm{H}\right\}$ NMR (Toluene-d $8,101 \mathrm{MHz}, 298 \mathrm{~K}$ ) spectrum between 35 and $75 \mathrm{ppm}$ of a reaction mixture in a J. Young NMR tube after $1155 \mathrm{~min}$ at $80^{\circ} \mathrm{C}$. Mesitylene $(18 \mu \mathrm{mol})$ was used as internal standard. 


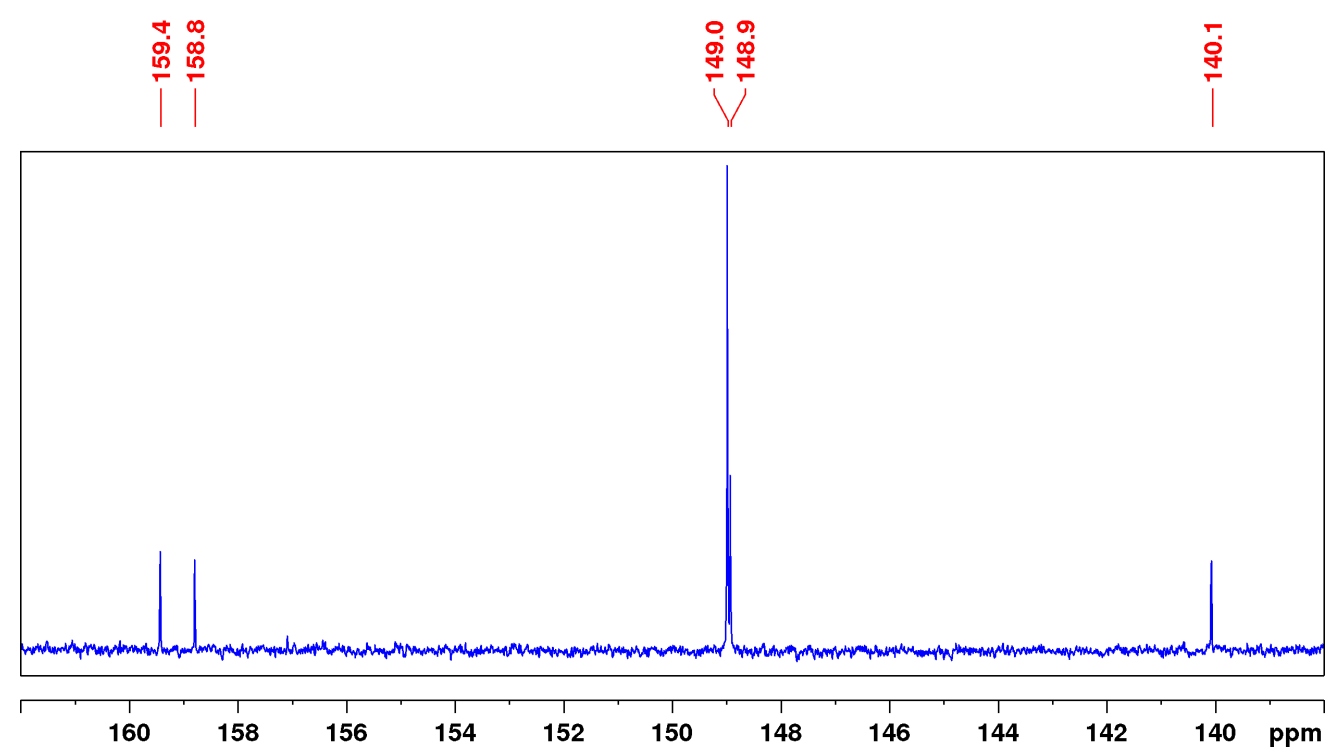

Figure S48: ${ }^{13} \mathrm{C}\left\{{ }^{1} \mathrm{H}\right\} \mathrm{NMR}$ (Toluene- $\mathrm{d}_{8}, 101 \mathrm{MHz}, 298 \mathrm{~K}$ ) spectrum between 138 and $162 \mathrm{ppm}$ of a reaction mixture in a J. Young NMR tube after $1155 \mathrm{~min}$ at $80^{\circ} \mathrm{C}$. Mesitylene $(18 \mu \mathrm{mol})$ was used as internal standard. 


\subsection{Reaction Monitoring via NMR without Internal Standard}

\subsubsection{NMR Spectra of Reaction Monitoring without Internal Standard in a J.} Young NMR Tube (Closed Atmosphere)

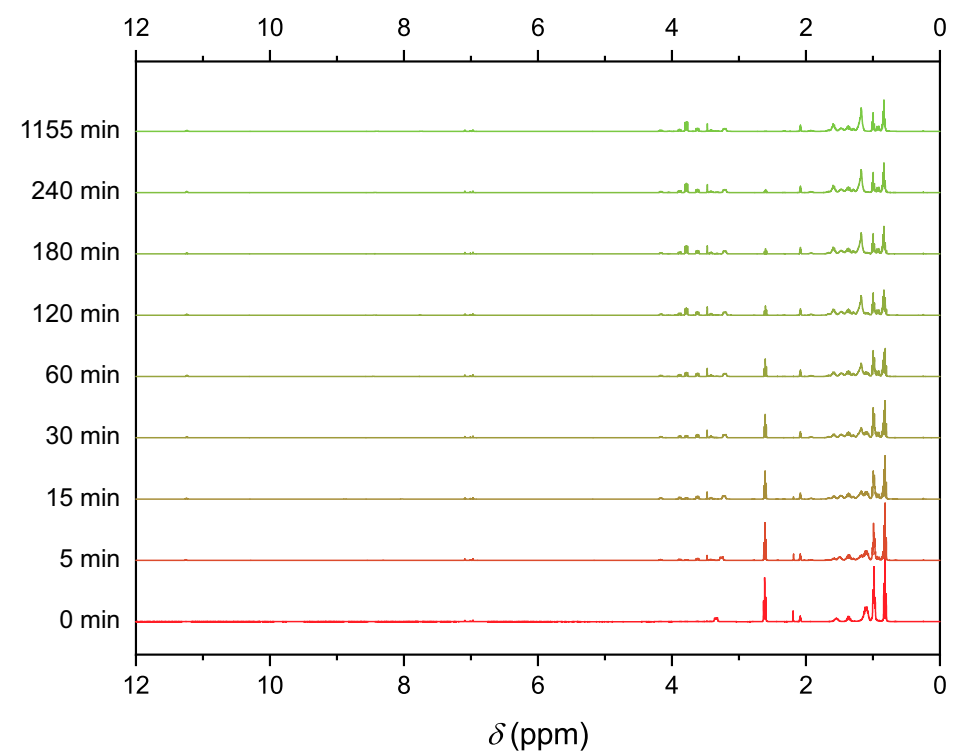

Figure S49: Overview of ${ }^{1} \mathrm{H}$ NMR (Toluene- $\mathrm{d}_{8}, 400 \mathrm{MHz}, 298 \mathrm{~K}$ ) spectra of a reaction mixture in a J. Young NMR tube without internal standard after defined time periods at $80^{\circ} \mathrm{C}$.

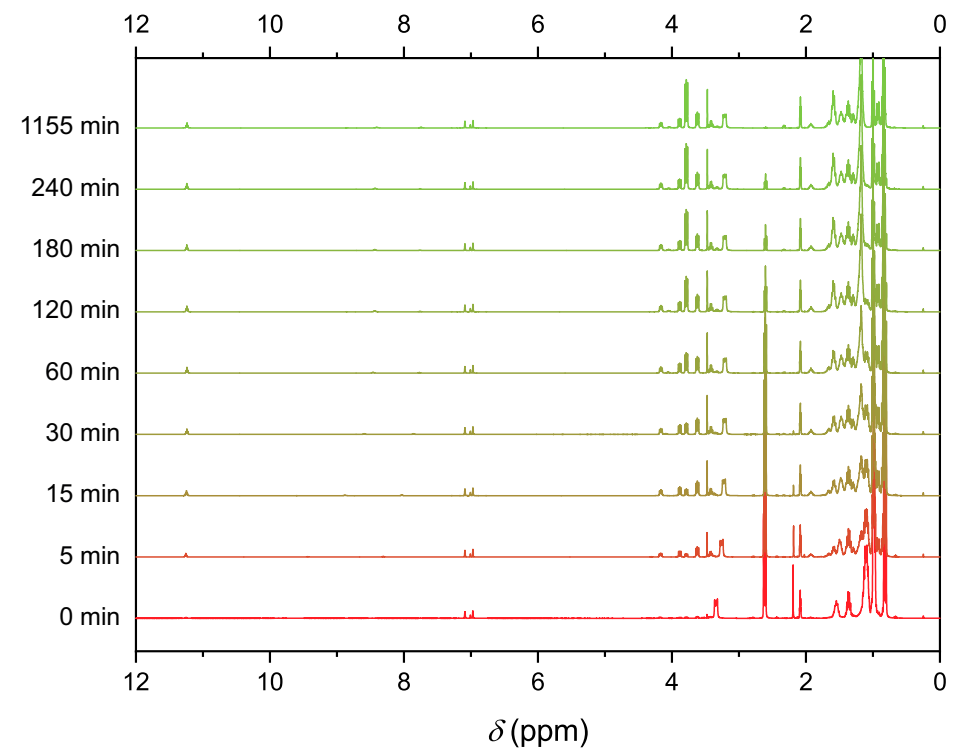

Figure S50: Overview of ${ }^{1} \mathrm{H}$ NMR (Toluene- $\mathrm{d}_{8}, 400 \mathrm{MHz}, 298 \mathrm{~K}$ ) spectra of a reaction mixture in a J. Young NMR tube without internal standard after defined time periods at $80^{\circ} \mathrm{C}$. Intensity increased by a factor of 5 compared to spectrum S49. 


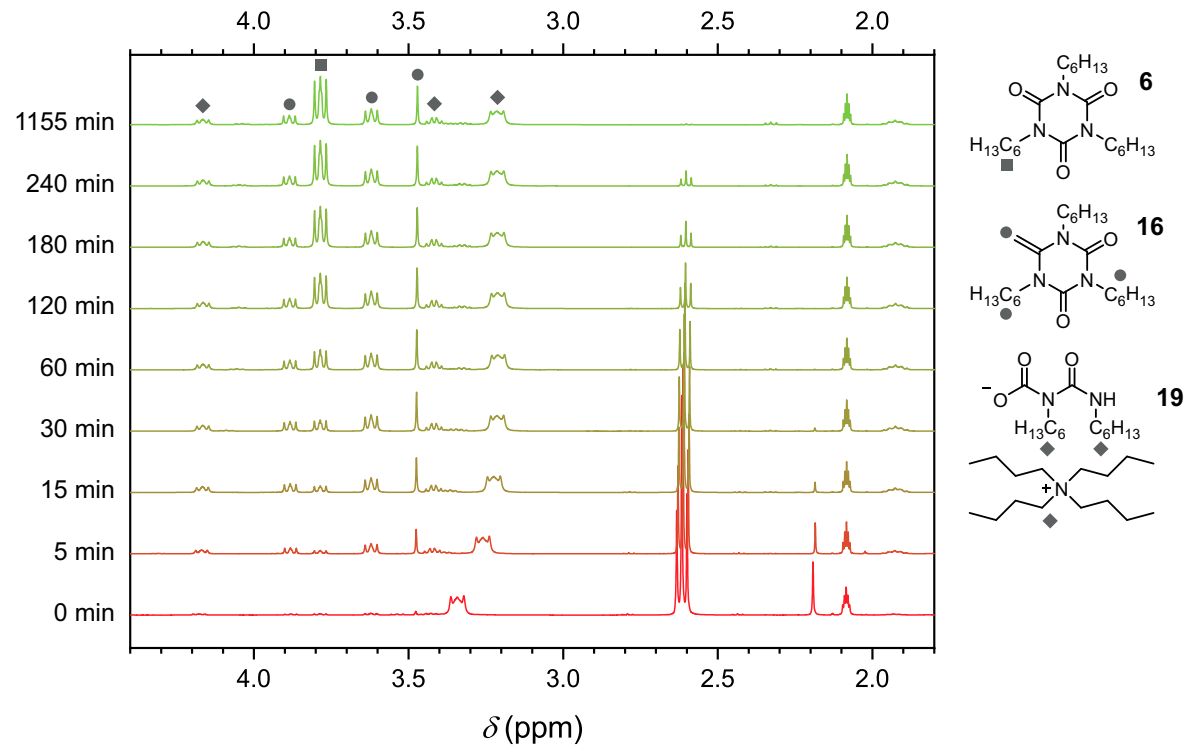

Figure S51: Overview between 1.8 and $4.4 \mathrm{ppm}$ of ${ }^{1} \mathrm{H}$ NMR (Toluene- $\mathrm{d}_{8}, 400 \mathrm{MHz}, 298 \mathrm{~K}$ ) spectra of a reaction mixture in a J. Young NMR tube without internal standard after defined time periods at $80^{\circ} \mathrm{C}$. Intensity increased by a factor of 5 compared to spectrum $\mathbf{S} 49$.

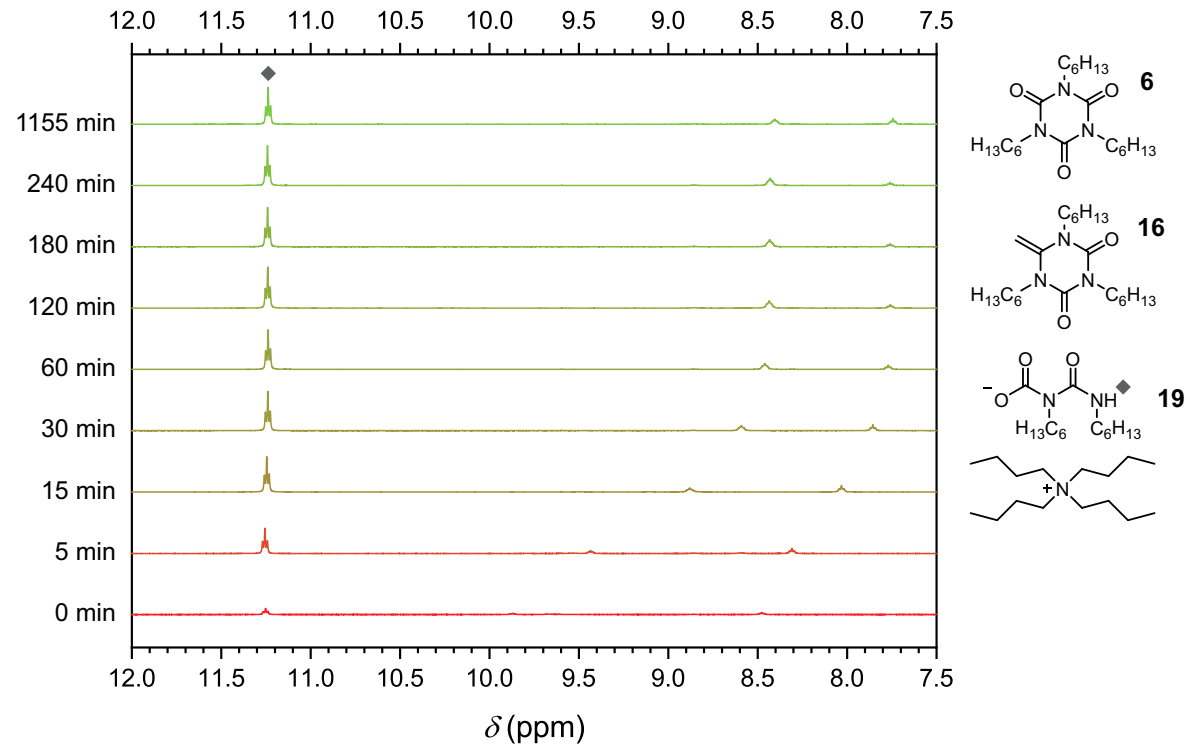

Figure S52: Overview between 7.5 and $12.0 \mathrm{ppm}$ of ${ }^{1} \mathrm{H}$ NMR (Toluene- $\mathrm{d}_{8}, 400 \mathrm{MHz}, 298 \mathrm{~K}$ ) spectra of a reaction mixture in a J. Young NMR tube without internal standard after defined time periods at $80^{\circ} \mathrm{C}$. Intensity increased by a factor of 35 compared to spectrum S49. 


\subsubsection{NMR Spectra of Reaction Monitoring without Internal Standard in an NMR} Tube (Open Atmosphere)

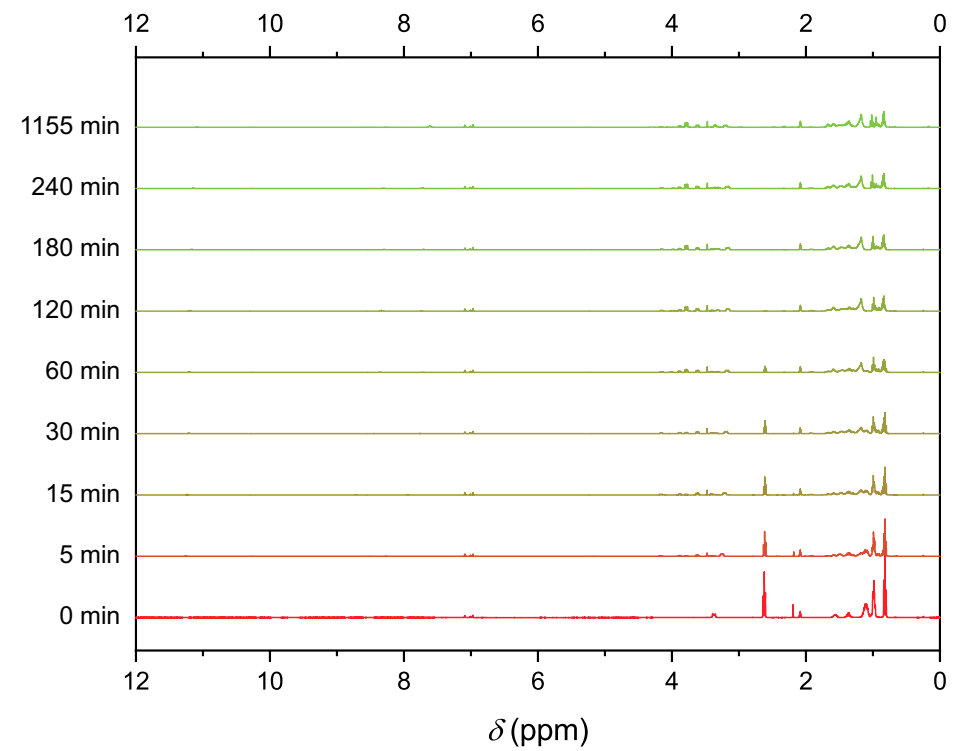

Figure S53: Overview of ${ }^{1} \mathrm{H}$ NMR (Toluene- $\mathrm{d}_{8}, 400 \mathrm{MHz}, 298 \mathrm{~K}$ ) spectra of a reaction mixture in an NMR tube without internal standard after defined time periods at $80^{\circ} \mathrm{C}$.

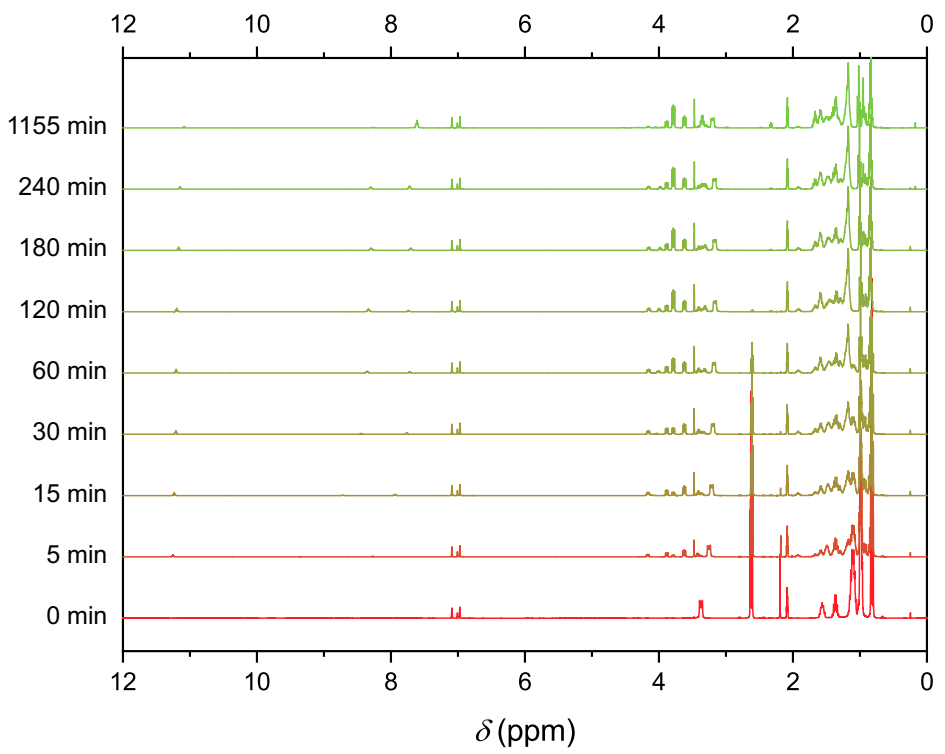

Figure S54: Overview of ${ }^{1} \mathrm{H}$ NMR (Toluene- $\mathrm{d}_{8}, 400 \mathrm{MHz}, 298 \mathrm{~K}$ ) spectra of a reaction mixture in an NMR tube without internal standard after defined time periods at $80^{\circ} \mathrm{C}$. Intensity increased by a factor of 5 compared to spectrum S53. 


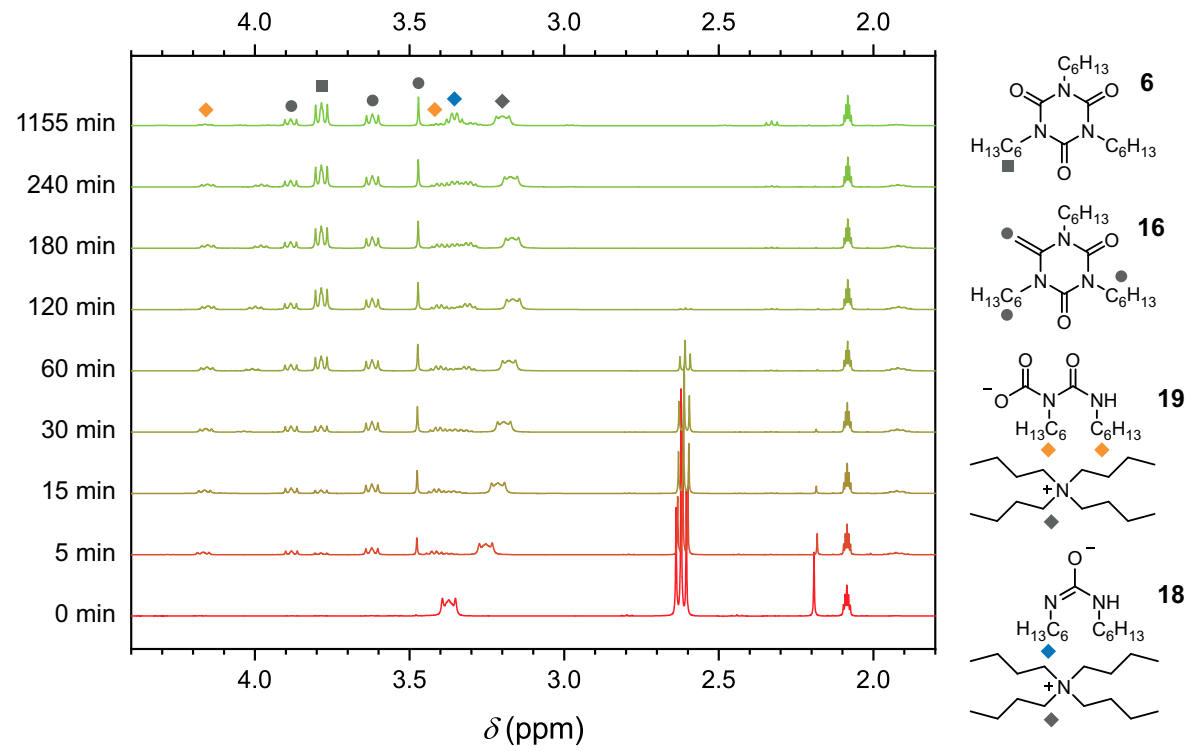

Figure S55: Overview between 1.8 and $4.4 \mathrm{ppm}$ of ${ }^{1} \mathrm{H}$ NMR (Toluene- $\mathrm{d}_{8}, 400 \mathrm{MHz}, 298 \mathrm{~K}$ ) spectra of a reaction mixture in an NMR tube without internal standard after defined time periods at $80^{\circ} \mathrm{C}$. Intensity increased by a factor of 5 compared to spectrum S53.

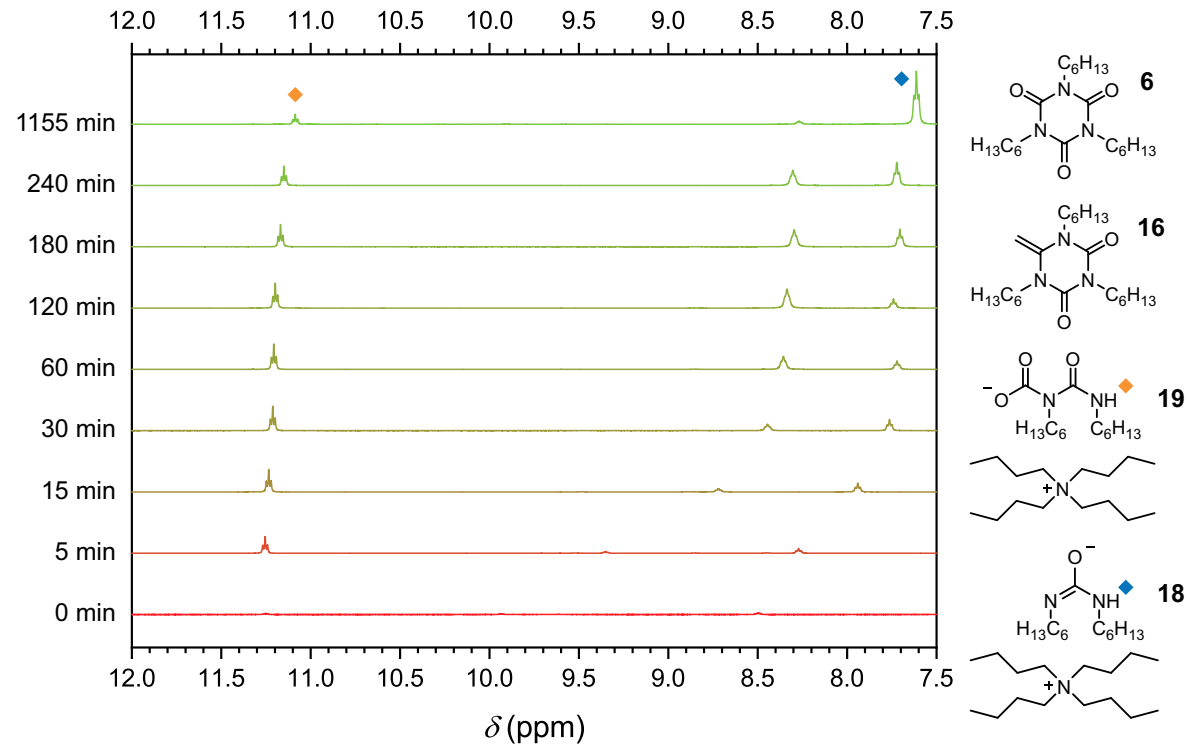

Figure S56: Overview between 7.5 and $12.0 \mathrm{ppm}$ of ${ }^{1} \mathrm{H}$ NMR (Toluene- $\mathrm{d}_{8}, 400 \mathrm{MHz}, 298 \mathrm{~K}$ ) spectra of a reaction mixture in an NMR tube without internal standard after defined time periods at $80^{\circ} \mathrm{C}$. Intensity increased by a factor of 35 compared to spectrum S53. 


\subsection{D NMR Spectra of Final Catalysis Mixtures}

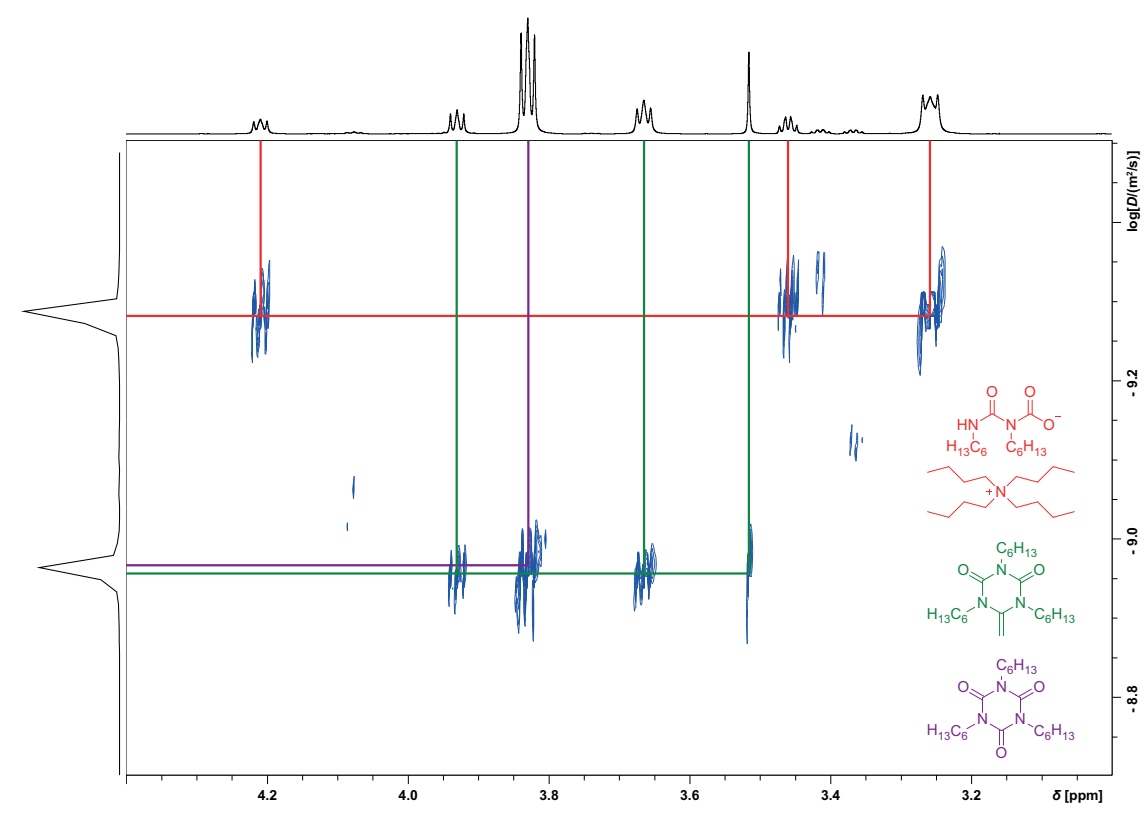

Figure S57: 2D DOSY (Toluene- $\mathrm{d}_{8}, 800 \mathrm{MHz}, 298 \mathrm{~K}$ ) spectrum between 3.0 and $4.4 \mathrm{ppm}$ (x-axis) of a reaction mixture in a J. Young NMR tube without internal standard after complete conversion at $80^{\circ} \mathrm{C}$.

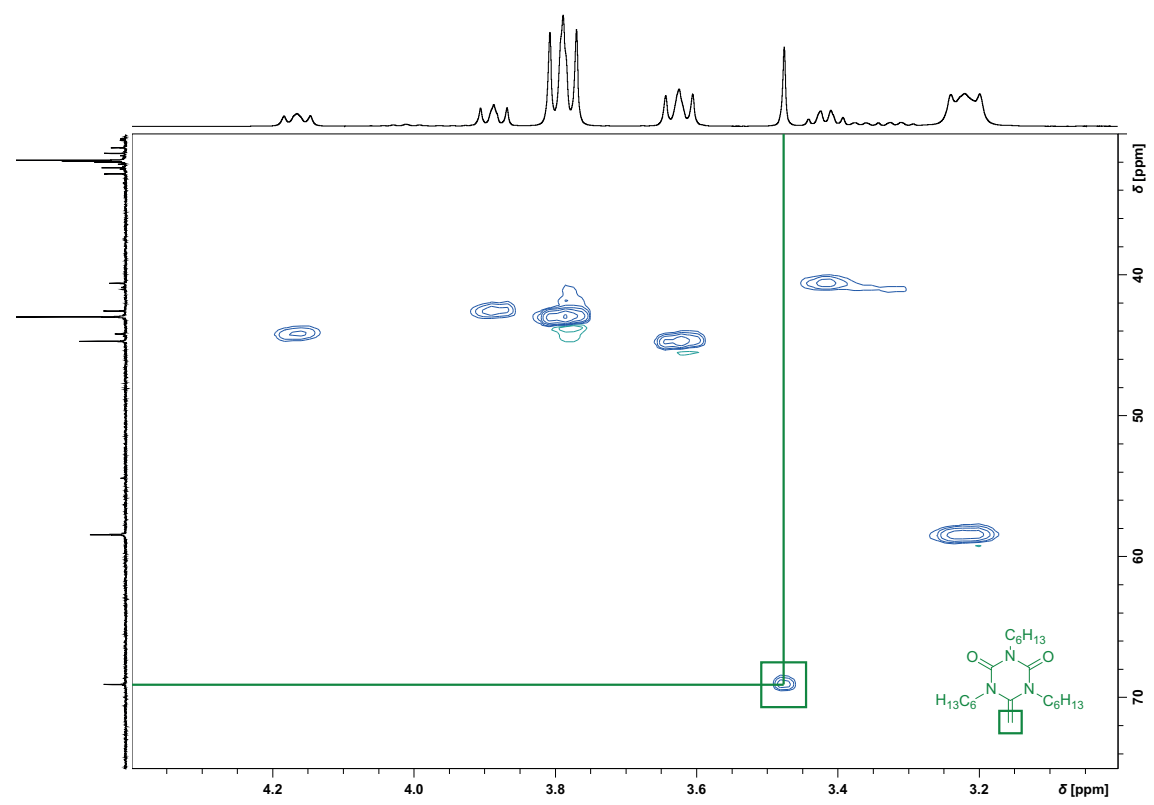

Figure S58: ${ }^{1} \mathrm{H}-{ }^{13} \mathrm{C}-\mathrm{HSQC}-\mathrm{ME}$ (Toluene- $\mathrm{d}_{8}, 400 / 101 \mathrm{MHz}, 298 \mathrm{~K}$ ) spectrum between 3.0 and 4.4 ppm (x-axis) of a reaction mixture in a J. Young NMR tube without internal standard after complete conversion at $80^{\circ} \mathrm{C}$. 


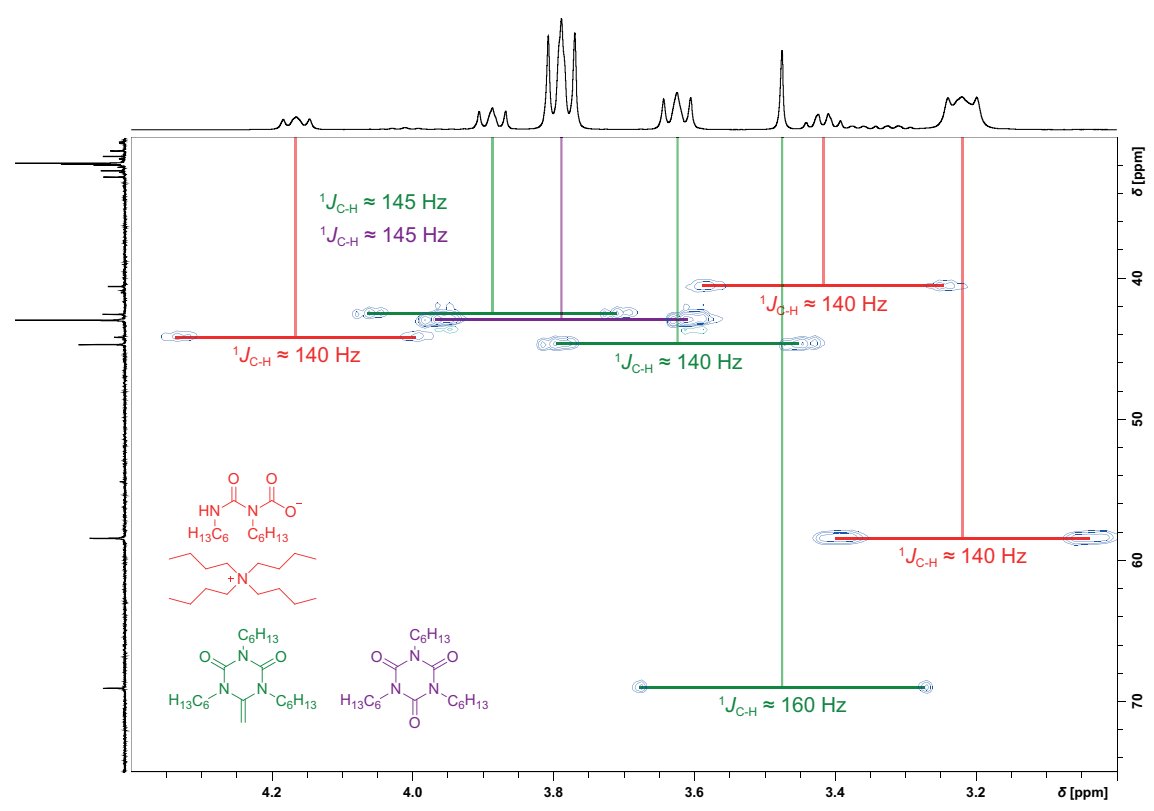

Figure S59: ${ }^{1} \mathrm{H}$-decoupled and ${ }^{13} \mathrm{C}$-coupled ${ }^{1} \mathrm{H}^{-13} \mathrm{C}$-HSQC-ME (Toluene- $\mathrm{d}_{8}, 400 / 101 \mathrm{MHz}, 298 \mathrm{~K}$ ) spectrum between 3.0 and $4.4 \mathrm{ppm}(\mathrm{x}$-axis) of a reaction mixture in a J. Young NMR tube without internal standard after complete conversion at $80^{\circ} \mathrm{C}$. The approximate coupling constants are given.

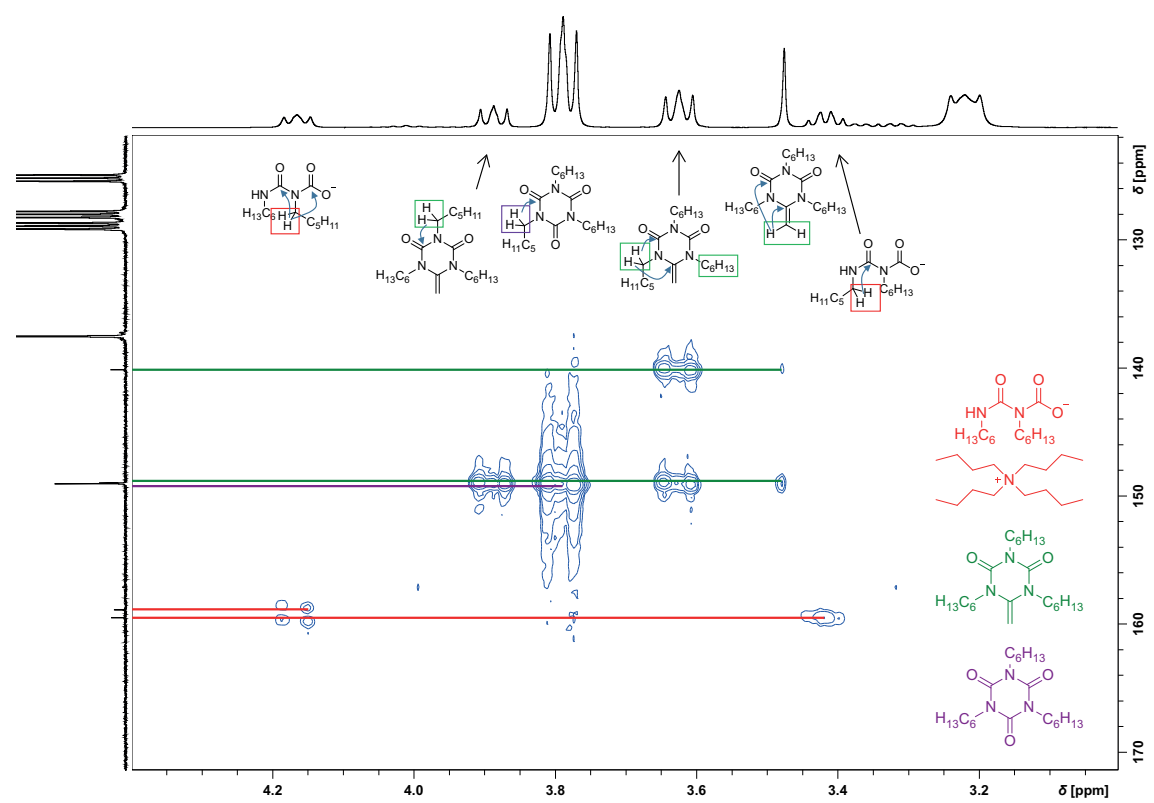

Figure S60: ${ }^{1} \mathrm{H}-{ }^{13} \mathrm{C}-\mathrm{HMBC}$ (Toluene- $\mathrm{d}_{8}, 400 / 101 \mathrm{MHz}, 298 \mathrm{~K}$ ) spectrum between 3.0 and $4.4 \mathrm{ppm}$ (x-axis) of a reaction mixture in a J. Young NMR tube without internal standard after complete conversion at $80^{\circ} \mathrm{C}$. 


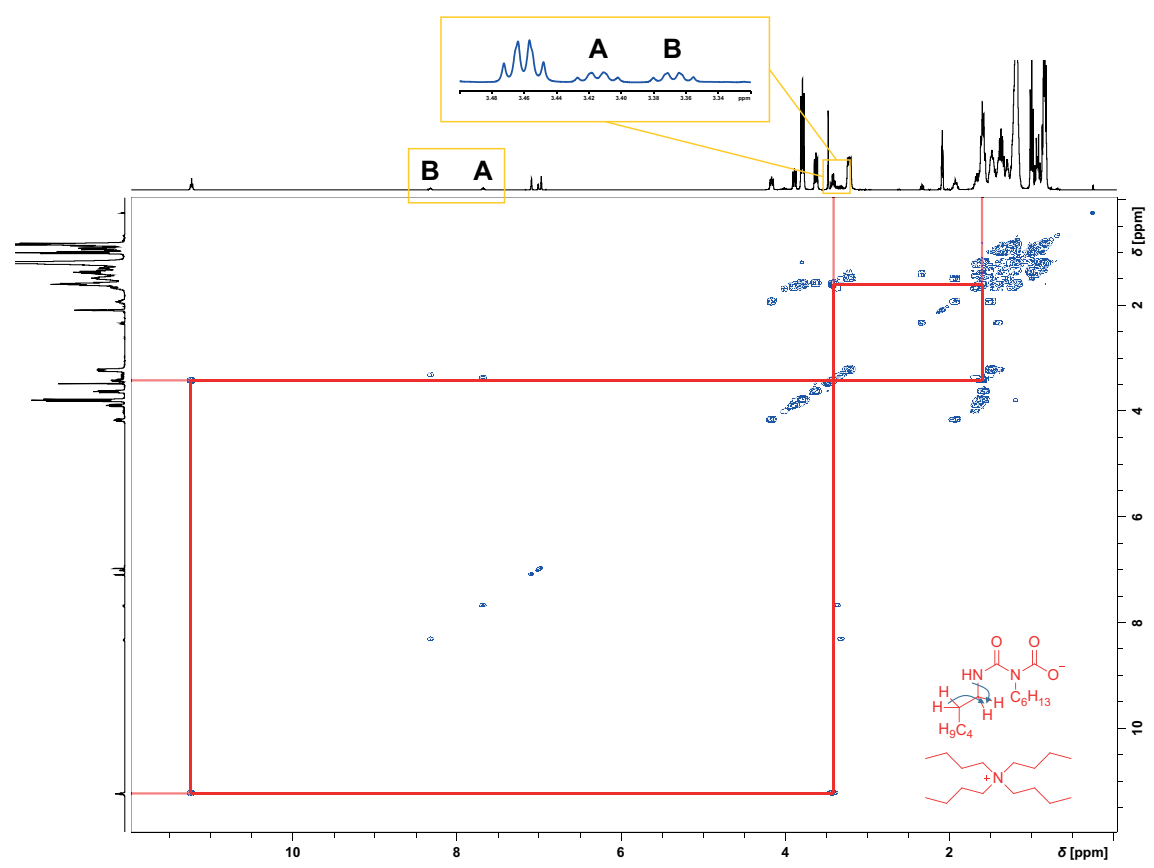

Figure S61: ${ }^{1} \mathrm{H}-{ }^{1} \mathrm{H}-\mathrm{COSY}$ (Toluene- $\mathrm{d}_{8}, 400 \mathrm{MHz}, 298 \mathrm{~K}$ ) spectrum between 0 and $12 \mathrm{ppm}$ of a reaction mixture in a J. Young NMR tube without internal standard after complete conversion at $80^{\circ} \mathrm{C}$. Structure A: Characteristic signals of the ureate obtained by decarboxylation of the amidocarbamate. Structure B: Presumably characteristic signals of the ureate-mono-isocyanate adduct. 


\section{Analysis of Reaction Mixtures via Orbitrap-MS}

4.1 Analysis after Short Reaction Time (15 min) 


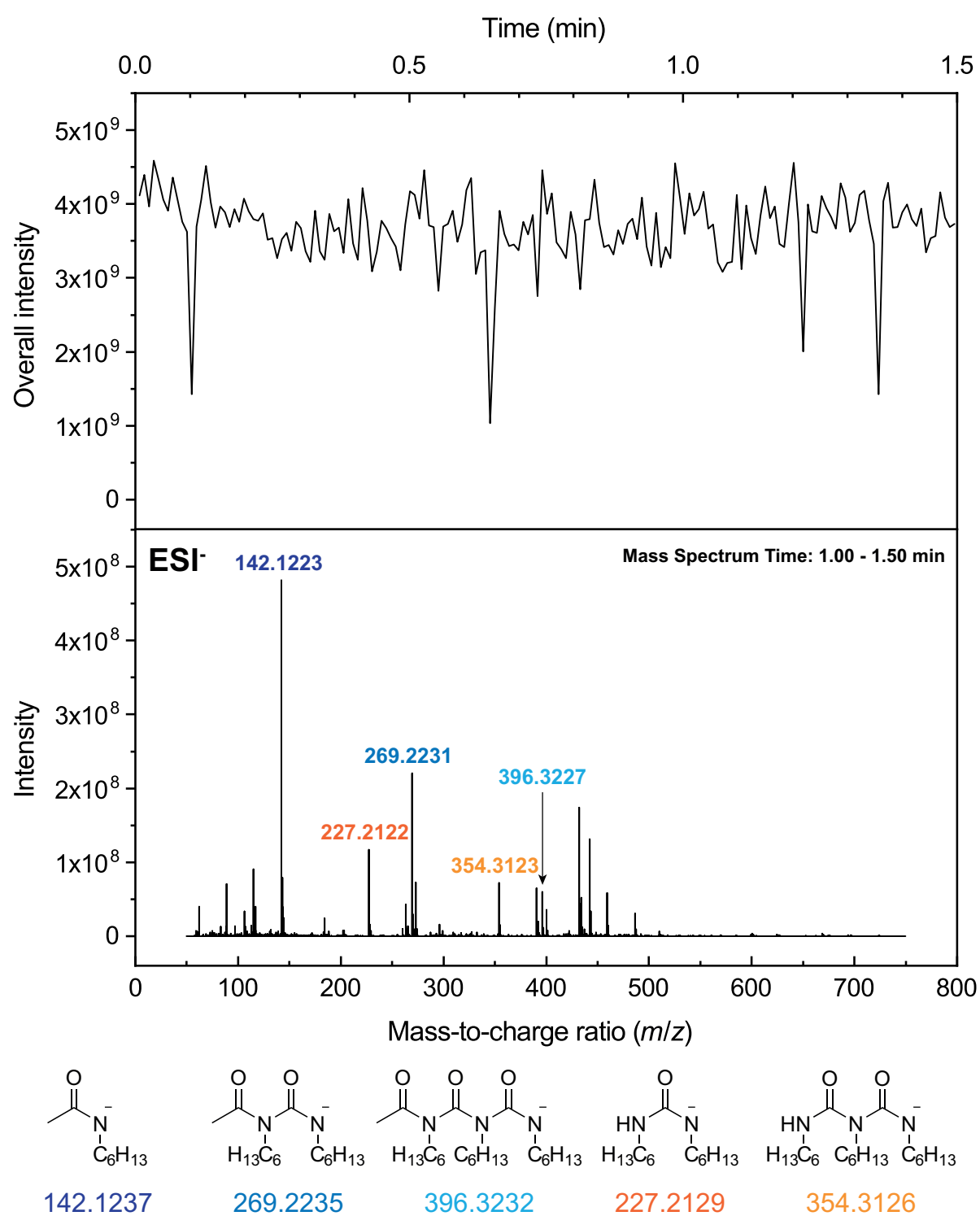

Figure S62: Orbitrap-MS analysis of a reaction mixture after $15 \mathrm{~min}$ in negative mode. The overall intensity during the measurement (top) and the mass spectrum averaged between 1.00 and $1.50 \mathrm{~min}$ (bottom) as well as the chemical structures referring to the relevant peaks in the mass spectrum are shown. 


\subsection{Analysis after Complete Reaction}
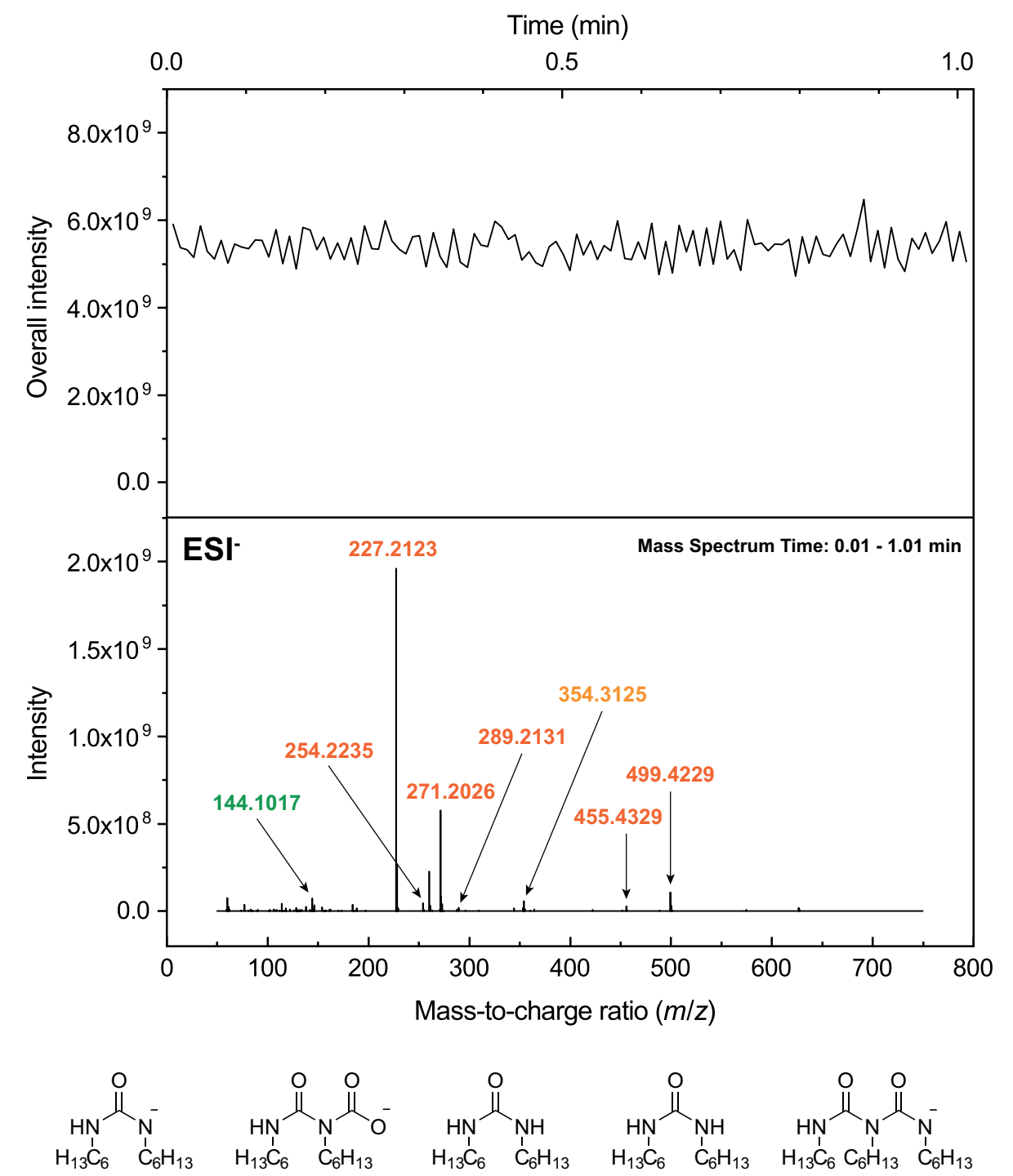

227.2129

271.2027

$\mathrm{HN}_{\mathrm{N}}^{\mathrm{O}} \mathrm{HCN}$<smiles>NC(=O)NC(=O)[O-]</smiles><smiles>[N]C(=O)NI</smiles><smiles>NC(=O)NC(=O)O</smiles>

$\mathrm{H}_{13} \mathrm{C}_{6} \mathrm{C}_{6} \mathrm{H}_{13} \quad \mathrm{C}_{6} \mathrm{H}_{13}$

$\mathrm{H}_{13} \mathrm{C}_{6} \quad \mathrm{C}_{6} \mathrm{H}_{13}$

$\begin{array}{lll}\mathrm{H}_{13} \mathrm{C}_{6} & \mathrm{I}_{6} \mathrm{H}_{13} & \mathrm{H}_{2} \mathrm{O}\end{array}$

$\mathrm{H}_{13} \mathrm{I}_{6} \quad \mathrm{I}_{6} \mathrm{H}_{13}$

$\mathrm{H}_{13} \mathrm{C}_{6} \quad \mathrm{I}_{6} \mathrm{H}_{13}$

254.2238

289.2133

455.4331

499.4229
354.3126

$\mathrm{H}_{13} \mathrm{C}_{6}{ }_{\mathrm{H}}^{\mathrm{O}} \mathrm{O}^{-}$

144.1030

Figure S63: Orbitrap-MS analysis of a final J. Young NMR reaction mixture in negative mode. The overall intensity during the measurement (top) and the mass spectrum averaged between 0.01 and $1.01 \mathrm{~min}$ (bottom) as well as the chemical structures referring to the relevant peaks in the mass spectrum are shown. 


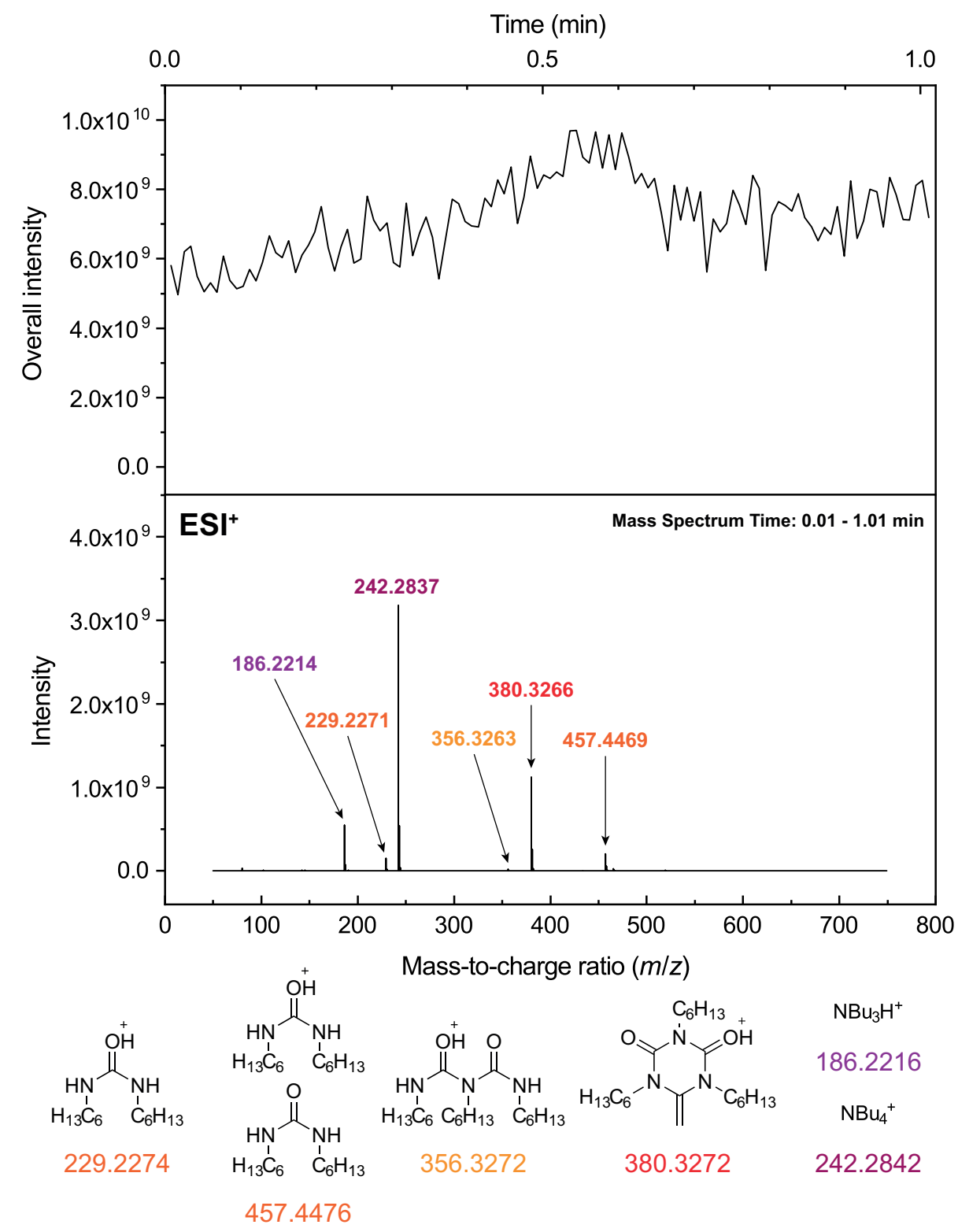

Figure S64: Orbitrap-MS analysis of a final J. Young NMR reaction mixture in positive mode. The overall intensity during the measurement (top) and the mass spectrum averaged between 0.01 and $1.01 \mathrm{~min}$ (bottom) as well as the chemical structures referring to the relevant peaks in the mass spectrum are shown. 


\subsection{Overview of the Exact Masses of all Intermediates}

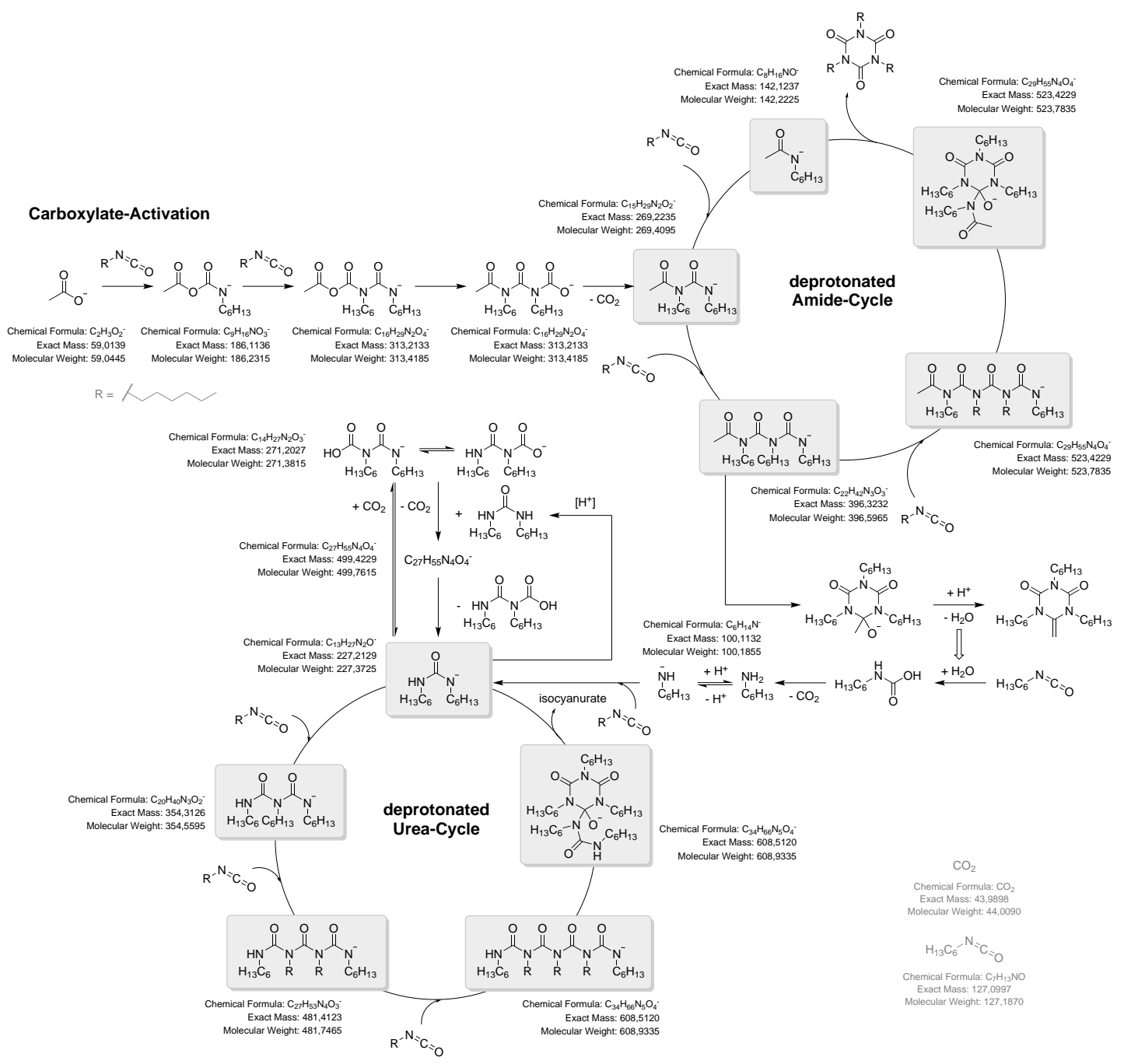

Figure S65: Overview of the exact masses of all intermediates. 


\section{Quantum Chemical Computations of Reaction Mechanisms}

\subsection{Overview of the Results}

An overview of the computed activation $\left(\Delta G^{\ddagger}\right)$ and reaction free energies $(\Delta G)$ is given in Table S2. Computed $\mathrm{p} K_{\mathrm{a}}$ values for some conjugated acids of the occurring species are given in Table S3. An overview of the computed activation $\left(\Delta G^{\ddagger}\right)$ and reaction free energies $(\Delta G)$ for two possible catalyst decomposition pathways (see Figure S66) is given in Table S4. 
Table S2: Overview of the computed activation and reaction free energies at $80^{\circ} \mathrm{C}$ in hexyl isocyanate on the B3LYP-D3/def2QZVP and M06-2X/def2-QZVP levels. All values are given in $\mathrm{kJ} / \mathrm{mol}^{a}$

\begin{tabular}{|c|c|c|c|c|}
\hline & $\Delta G^{\ddagger}(\mathrm{B} 3 \mathrm{LYP})$ & $\Delta G(\mathrm{~B} 3 \mathrm{LYP})$ & $\Delta G^{\neq}(\mathrm{M} 06-2 \mathrm{X})$ & $\Delta G(\mathrm{M} 06-2 \mathrm{X})$ \\
\hline \multicolumn{5}{|l|}{ Carboxylate Catalysis } \\
\hline $1+2 \longrightarrow 3$ & 86 & 55 & 88 & 42 \\
\hline $3+2 \longrightarrow 4$ & 61 & -1 & 60 & -22 \\
\hline $4+2 \longrightarrow 5$ & 100 & 11 & 105 & -8 \\
\hline $5 \longrightarrow 6+1$ & 74 & -188 & 81 & -174 \\
\hline $4 \longrightarrow 24+1$ & 112 & -36 & 123 & -20 \\
\hline \multicolumn{5}{|l|}{ Pre-Catalyst Activation } \\
\hline $4 \longrightarrow 7$ & - & -70 & - & -70 \\
\hline $7 \longrightarrow 8+\mathrm{CO}_{2}$ & - & 4 & - & 18 \\
\hline \multicolumn{5}{|l|}{ Depr. Amide Catalysis } \\
\hline $12+2 \longrightarrow 8$ & 67 & -25 & 71 & -40 \\
\hline $8+2 \longrightarrow 9$ & 61 & 1 & 65 & -11 \\
\hline $9+2 \longrightarrow 10$ & 79 & 2 & 78 & -9 \\
\hline $10 \longrightarrow 11$ & 64 & -28 & 61 & -50 \\
\hline $11 \longrightarrow 6+12$ & 26 & -72 & 37 & -53 \\
\hline $9 \longrightarrow 24+12$ & 121 & 43 & 125 & 47 \\
\hline \multicolumn{5}{|l|}{ Catalyst Migration } \\
\hline $9 \longrightarrow 13$ & 62 & -15 & 59 & -32 \\
\hline $2+\mathrm{H}_{2} \mathrm{O}+12 \longrightarrow 17^{*}+12+\mathrm{H}^{+}$ & 75 & -40 & 81 & -56 \\
\hline $17^{*} \longrightarrow 17$ & - & -56 & - & -51 \\
\hline $13+2 \longrightarrow 14$ & 86 & 24 & 88 & 1 \\
\hline $14+12+\mathrm{H}^{+} \longrightarrow 15+12$ & - & -1 & - & 3 \\
\hline $15+12 \longrightarrow 16+17+12+\mathrm{H}^{+}$ & 95 & -30 & 121 & -18 \\
\hline $17+2 \longrightarrow 18+\mathrm{CO}_{2}$ & 74 & 21 & 77 & 16 \\
\hline \multicolumn{5}{|l|}{ Depr. Urea Catalysis } \\
\hline $18+\mathrm{CO}_{2} \longrightarrow 19$ & - & -84 & - & -94 \\
\hline $18+2 \longrightarrow 20$ & 83 & -59 & 95 & -70 \\
\hline $20+2 \longrightarrow 21$ & 112 & 15 & 118 & 11 \\
\hline $21+2 \longrightarrow 22$ & 80 & 10 & 76 & -12 \\
\hline $22 \longrightarrow 23$ & 78 & -50 & 83 & -64 \\
\hline $23 \longrightarrow 6+18$ & 31 & -39 & 45 & -28 \\
\hline $21 \longrightarrow 24+18$ & 106 & 62 & 100 & 59 \\
\hline
\end{tabular}

$a$ The first column gives the calculated transformation together with the respective part of catalysis in which the transformation takes place. The numbers of the species refer to the numbering in the manuscript. 
Table S3: Computed $\mathrm{p} K_{\mathrm{a}}$ values in hexyl isocyanate for some conjugated acids of the occurring species on the B3LYPD3/def2-QZVP and M06-2X/def2-QZVP levels. The experimental $\mathrm{pK}$ a value for acetic acid in DMSO is provided in parentheses. ${ }^{a}$

\begin{tabular}{|c|c|c|c|}
\hline \multirow[b]{2}{*}{ Carboxylate Catalysis } & \multirow[t]{2}{*}{ Structure } & \multirow[t]{2}{*}{$\mathrm{p} K_{\mathrm{a}}(\mathrm{B} 3 \mathrm{LYP})$} & \multirow[t]{2}{*}{$\mathrm{p} K_{\mathrm{a}}(\mathrm{M} 06-2 \mathrm{X})$} \\
\hline & & & \\
\hline $1+\mathrm{H}^{+}$ & & $10.1(12.3)$ & $12.6(12.3)$ \\
\hline \multicolumn{4}{|l|}{ Depr. Amide Catalysis } \\
\hline $12+\mathrm{H}^{+}$ & & 22.6 & 23.9 \\
\hline $8+\mathrm{H}^{+}$ & $\begin{array}{l}\mathrm{N} \\
\mathrm{R}\end{array}$ & 18.0 & 19.4 \\
\hline $9+\mathrm{H}^{+}$ & $\begin{array}{rr}H_{N} & N \\
R & R\end{array}$ & 13.0 & 1.8 \\
\hline 14 & & 13.9 & 16.8 \\
\hline
\end{tabular}

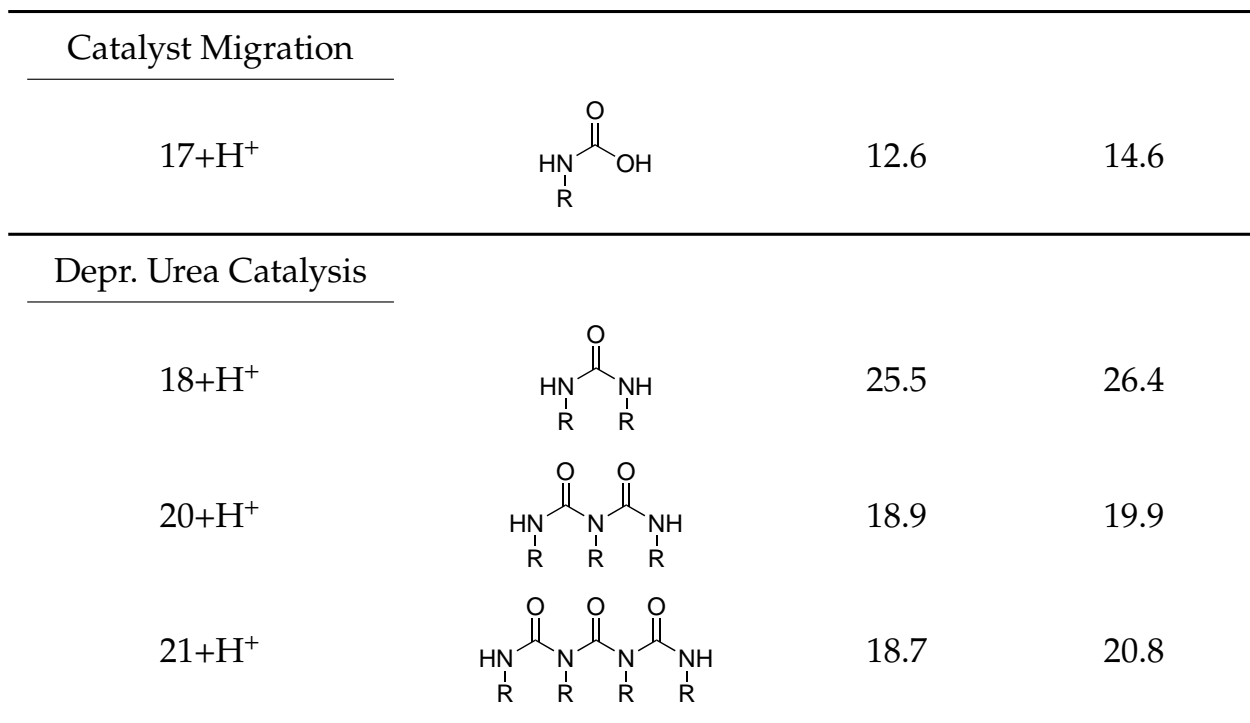

${ }^{a}$ The first column gives the species for which the deprotonation was calculated together with the respective part of catalysis in which the species occurs. The numbers of the species refer to the numbering in the manuscript. 


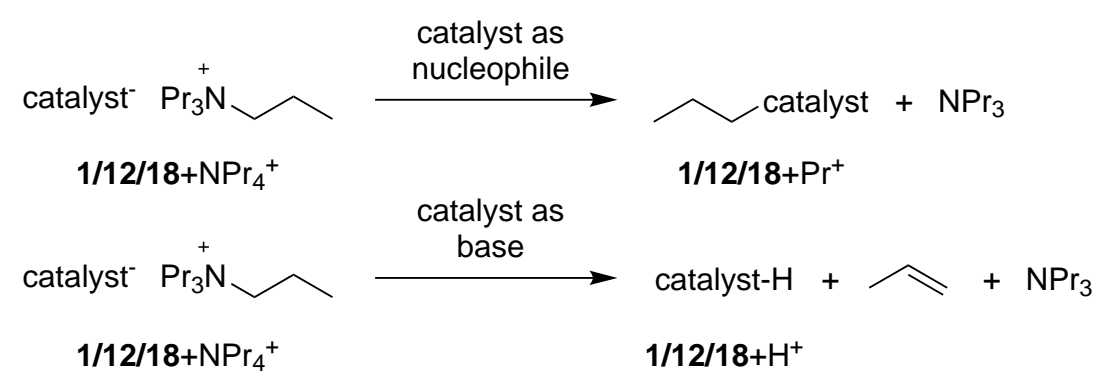

Figure S66: Two possible catalyst decomposition pathways.

Table S4: Overview of the computed activation and reaction free energies for the two possible catalyst decomposition pathways at $80^{\circ} \mathrm{C}$ in hexyl isocyanate on the B3LYP-D3/def2-QZVP and M06-2X/def2-QZVP levels. All values are given in $\mathrm{kJ} / \mathrm{mol}$.

\begin{tabular}{cccccc}
\hline Catalyst & Acts as... & $\Delta G^{\ddagger}(\mathrm{B} 3 \mathrm{LYP})$ & $\Delta G(\mathrm{~B} 3 \mathrm{LYP})$ & $\Delta G^{ \pm}(\mathrm{M} 06-2 \mathrm{X})$ & $\Delta G(\mathrm{M} 06-2 \mathrm{X})$ \\
\hline \multirow{2}{*}{ Carboxylate 1 } & nucleophile & 145 & -60 & 168 & -69 \\
& base & 145 & -68 & 161 & -65 \\
\hline \multirow{2}{*}{ Depr. Amide 12 } & nucleophile & 131 & -135 & 148 & -135 \\
& base & 140 & -141 & 150 & -126 \\
\hline \multirow{2}{*}{ Depr. Urea 18 } & nucleophile & 141 & -173 & 170 & -173 \\
& base & 130 & -172 & 137 & -158 \\
\hline
\end{tabular}




\subsection{Cartesian Coordinates Catalytic Cycles}

All coordinates are given in Ångström in xyz format. The energy given is the BP86-D3cosmo/def2-TZVP energy of the optimized structure. The energy is given in Hartree. The first lines of the cartesian coordinate inputs refer to the number of atoms.

\subsubsection{General Compounds}

\section{Compound 2 (Isocyanate)}

13

Energy $=-286.7708394088$

C
$-0.5965061$
$-0.0654260$
$-2.0230095$
0.7661924
$-0.0977577$
$-1.3312065$
0.1334330
0. 1001381
0.0602483
1.0076730
0.0384424
1.9920839
$-0.3004470-3.5282851$
$0.6712918-1.7515876$
1.2576921
$-1.0702767-1.4902864$
$0.9089766-1.8288053$
$-0.8360804-1.5720911$
$-0.2739382-4.0135923$
$0.4715274-3.9978638$
$-0.0119286$
$-1.2800025-3.7401755$

C

$\mathrm{N} \quad 0.6865422$

C $\quad-0.0996811$

$0 \quad-0.7681156$

C $\quad-0.4657113$

$\mathrm{H} \quad 1.4302693$

$\mathrm{H} \quad-1.0709895$

$\mathrm{H} \quad-1.2417301$

$\mathrm{H} \quad-1.4508029$

H $\quad 0.1618743$

$\mathrm{H}$

\section{Compound 6 (Isocyanurate)}

\begin{tabular}{|c|c|c|c|}
\hline \multicolumn{4}{|c|}{ Energy $=-860.4104357833$} \\
\hline $\mathrm{N}$ & 0.0938111 & Q. 1079673 & 1.7201872 \\
\hline $\mathrm{C}$ & -1.0919594 & -0.2065735 & 2.3760230 \\
\hline $\mathrm{N}$ & -0.9727762 & -0.5025227 & 3.7299991 \\
\hline C & 0.2269274 & -0.4796525 & 4.4338681 \\
\hline $\mathrm{N}$ & 1.3618350 & -0.1908031 & 3.6831300 \\
\hline
\end{tabular}




\begin{tabular}{|c|c|c|c|}
\hline & 1.3456298 & 0.1180680 & 2.3269283 \\
\hline & -2.1746079 & -0.2189910 & 1.7976435 \\
\hline & -2.2053565 & -0.8051981 & 4.4808527 \\
\hline & -2.8518235 & 0.4502198 & 5.0655303 \\
\hline & 0.2819862 & -0.7112020 & 5.6379866 \\
\hline & 2.6592314 & -0.1536509 & 4.3832785 \\
\hline & 2.9752625 & 1.2318191 & 4.9456417 \\
\hline & 2.3701059 & 0.3858397 & 1.7059069 \\
\hline & 0.0248529 & 0.4074047 & 0.2778023 \\
\hline & 0.1717763 & -0.8477756 & -0.5815232 \\
\hline & -0.9418885 & 0.8916041 & 0.1006050 \\
\hline & 0.8263474 & 1.1234051 & 0.0645213 \\
\hline & 2.6077595 & -0.8985350 & 5.1851418 \\
\hline & 3.4192328 & -0.4636876 & 3.6575912 \\
\hline & -2.8854554 & -1.3067164 & 3.7833934 \\
\hline & -1.9280582 & -1.5076417 & 5.2747538 \\
\hline & 0.0998131 & -0.5113900 & -2.0713594 \\
\hline & 1.1322765 & -1.3315878 & -0.347 \\
\hline & -0.6238186 & -1.5598419 & -0.3138554 \\
\hline & -4.1230867 & 0.1075861 & 5.8430328 \\
\hline & -2.1269699 & 0.9520788 & 5.7245130 \\
\hline & -3.0836267 & 1.1482298 & 4.2465785 \\
\hline & 4.3209632 & 1.2409126 & 5.6716330 \\
\hline & 2.9835688 & 1.9599333 & 4.1202134 \\
\hline t & 2.1701706 & 1.5307446 & 5.6340088 \\
\hline & -4.5833051 & 1.0131401 & 6.2609642 \\
\hline & -4.8658238 & -0.3796273 & 5.1936937 \\
\hline & -3.9050534 & -0.5761954 & 6.6769641 \\
\hline$f$ & 0.2062739 & -1.4172843 & -2.6833757 \\
\hline F & -0.8627523 & -0.0431909 & -2.3262011 \\
\hline & 0.9002290 & 0.1864697 & -2.3592393 \\
\hline & 4.5425497 & 2.2384570 & 6.0745205 \\
\hline & 4.3227431 & 0.5296675 & 6.5111229 \\
\hline
\end{tabular}


$\begin{array}{llll}\mathrm{H} & 5.1400359 & 0.9615755 & 4.9921730\end{array}$

\section{Compound 24 (Uretdione)}

\begin{tabular}{|c|c|c|c|}
\hline \multicolumn{4}{|c|}{ Energy $=-573.5597655830$} \\
\hline $\mathrm{C}$ & -0.0304338 & -0.3112365 & Q. 1977483 \\
\hline N & -1.3048122 & -0.5526796 & -0.4692711 \\
\hline C & -2.6373889 & -0.4266780 & 0.0185952 \\
\hline 0 & -3.1625904 & -0.6129604 & 1.0890580 \\
\hline C & -1.7731041 & -0.1192778 & -1.7430945 \\
\hline $\mathrm{N}$ & -3.1053746 & 0.0057330 & -1.2552332 \\
\hline 0 & -1.2478208 & 0.0680999 & -2.8133235 \\
\hline C & -4.3810219 & -0.2293978 & -1.9216582 \\
\hline $\mathrm{H}$ & 0.7588676 & -0.6743866 & -0.4758051 \\
\hline $\mathrm{H}$ & -0.0099729 & -0.9489328 & 1.0930862 \\
\hline$c$ & Q. 1967729 & 1.1571821 & 0.5658428 \\
\hline $\mathrm{H}$ & Q. 1458691 & 1.7680147 & -0.3494412 \\
\hline $\mathrm{C}$ & 1.5436944 & 1.3621512 & 1.2599025 \\
\hline $\mathrm{H}$ & -0.6221490 & 1.4931471 & 1.2214335 \\
\hline $\mathrm{H}$ & 2.3750629 & 1.0508378 & 0.6100184 \\
\hline $\mathrm{H}$ & 1.6039751 & 0.7751262 & 2.1884635 \\
\hline $\mathrm{H}$ & 1.6953356 & 2.4188239 & 1.5186887 \\
\hline $\mathrm{H}$ & -4.4000918 & 0.4106620 & -2.8153345 \\
\hline $\mathrm{H}$ & -5.1683701 & 0.1347862 & -1.2463609 \\
\hline $\mathrm{C}$ & -4.6139148 & -1.6960640 & -2.2933062 \\
\hline $\mathrm{H}$ & -3.7970944 & -2.0330965 & -2.9509496 \\
\hline $\mathrm{C}$ & -5.9625569 & -1.8942605 & -2.9859545 \\
\hline $\mathrm{H}$ & -4.5638301 & -2.3094384 & -1.3796872 \\
\hline $\mathrm{H}$ & -6.0220023 & -1.3046572 & -3.9129379 \\
\hline $\mathrm{H}$ & -6.7918528 & -1.5815358 & -2.3341033 \\
\hline $\mathrm{H}$ & -6.1185060 & -2.9497121 & -3.2471561 \\
\hline
\end{tabular}




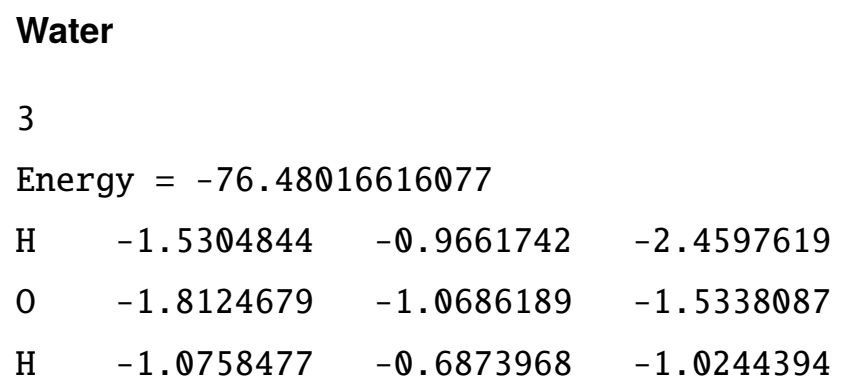

\subsubsection{Carboxylate Catalysis}

\section{Compound 1}

\begin{tabular}{lrrr}
7 & \\
\multicolumn{4}{l}{ Energy $=-228.7559125748$} \\
$\mathrm{H}$ & -4.5144755 & 0.6745488 & 6.9789047 \\
$\mathrm{H}$ & -4.7600117 & -0.8194952 & 6.0651513 \\
$\mathrm{C}$ & -4.5137625 & 0.2479263 & 5.9645855 \\
$\mathrm{O}$ & -2.1570769 & -0.1678598 & 5.9448849 \\
$\mathrm{H}$ & -5.2893837 & 0.7432241 & 5.3670411 \\
$\mathrm{C}$ & -3.1166323 & 0.4044443 & 5.3402179 \\
$\mathrm{O}$ & -3.0142774 & 1.0893014 & 4.2765646
\end{tabular}

\section{Compound $1+\mathrm{H}^{+}$}

8

Energy $=-229.2202348430$

$\begin{array}{lrrr}\text { C } & -4.5109850 & 0.2482695 & 5.9651375 \\ \text { C } & -3.1170160 & 0.3557830 & 5.4160106 \\ \text { O } & -3.0579135 & 1.0814025 & 4.2730800 \\ \text { O } & -2.1239012 & -0.1455491 & 5.9237939 \\ \text { H } & -4.5241765 & 0.6728735 & 6.9781269 \\ \text { H } & -4.7759893 & -0.8141444 & 6.0504915 \\ \text { H } & -5.2442125 & 0.7645459 & 5.3381820 \\ \text { H } & -2.1197791 & 1.1040111 & 3.9822966\end{array}$




\section{Compound 3}

$\begin{array}{lrrr}20 & & \\ \text { Energy }=-515.5234336838 & \\ \mathrm{H} & 0.0210349 & 0.5005252 & -2.1071285 \\ \mathrm{O} & -1.3109504 & -2.4588582 & 2.2952893 \\ \mathrm{O} & -3.4832240 & -0.9513155 & 0.3997486 \\ \mathrm{H} & 0.5527579 & 0.7119195 & 0.3251921 \\ \mathrm{C} & 0.3831980 & -0.3668487 & -1.5307421 \\ \mathrm{H} & 2.4434306 & 0.3362753 & -1.3637799 \\ \mathrm{~N} & -1.4138070 & 0.0710639 & 0.1509520 \\ \mathrm{C} & -2.2549323 & -0.8966442 & 0.2505432 \\ \mathrm{H} & 2.1424638 & -0.6770014 & -2.7922221 \\ \mathrm{H} & -0.1547173 & -1.2490003 & -1.9122108 \\ \mathrm{C} & 0.0076359 & -0.1688580 & -0.0561578 \\ \mathrm{C} & 1.8895947 & -0.5417477 & -1.7308444 \\ \mathrm{C} & -1.2469117 & -2.9604462 & 1.1706804 \\ \mathrm{O} & -1.6378294 & -2.3524313 & 0.0573598 \\ \mathrm{H} & -1.5888560 & -5.0383984 & 0.8029543 \\ \mathrm{H} & 0.3764172 & -1.0341510 & 0.5248581 \\ \mathrm{H} & 2.2613662 & -1.4215709 & -1.1832438 \\ \mathrm{C} & -0.7307121 & -4.3588142 & 0.9153070 \\ \mathrm{H} & -0.1241046 & -4.6973629 & 1.7621142 \\ \mathrm{H} & -0.1478883 & -4.4020146 & -0.0129010\end{array}$

\section{Compound 4}

33
$\begin{array}{lrrr}\text { Energy }=-802.3108387660 & \\ \mathrm{H} & 2.4133015 & 3.1435838 & 1.3897297 \\ \mathrm{O} & -0.6521592 & -0.1098339 & 2.5635522 \\ \mathrm{H} & 0.1260365 & 1.8655869 & 1.3907469 \\ \mathrm{C} & 2.6576099 & 2.6206690 & 0.4525894 \\ \mathrm{H} & 3.7493993 & 2.5052761 & 0.4070289\end{array}$




$\begin{array}{rrrr}\mathrm{H} & 2.2838838 & 0.6172130 & 1.2064021 \\ \mathrm{C} & -0.7281895 & -0.4809156 & 1.3958211 \\ \mathrm{C} & 0.4333679 & 1.4116231 & 0.4396209 \\ \mathrm{H} & 2.3523494 & 3.2701180 & -0.3818428 \\ \mathrm{C} & 1.9548227 & 1.2646399 & 0.3781815 \\ \mathrm{O} & 0.2690968 & -2.9628257 & 1.6057579 \\ \mathrm{H} & 0.0735441 & 2.0487701 & -0.3810725 \\ \mathrm{H} & -2.6697256 & -3.9243152 & 0.9429634 \\ \mathrm{O} & -1.5014830 & -1.6364055 & 1.0742977 \\ \mathrm{~N} & -0.2513274 & 0.1233021 & 0.3025139 \\ \mathrm{C} & -0.8307538 & -2.8345103 & 1.1051232 \\ \mathrm{H} & 2.2243253 & 0.7458383 & -0.5552514 \\ \mathrm{C} & -1.6665742 & -3.9185276 & 0.4958035 \\ \mathrm{H} & -1.1837240 & -4.8900311 & 0.6364964 \\ \mathrm{C} & -0.4520453 & -0.3813885 & -1.0957159 \\ \mathrm{H} & -1.7797911 & -3.7096731 & -0.5767154 \\ \mathrm{O} & -1.0839914 & 0.4128417 & -1.8503072 \\ \mathrm{~N} & \mathbb{0 . 0 8 6 4 1 1 2} & -1.5486773 & -1.3095774 \\ \mathrm{C} & -0.1452435 & -2.0808928 & -2.6458440 \\ \mathrm{H} & -1.2292305 & -2.2456902 & -2.8235324 \\ \mathrm{H} & \mathbb{0 . 1 6 9 7 6 5 8} & -1.3606315 & -3.4291876 \\ \mathrm{H} & \mathbb{0 . 2 8 5 4 5 4 7} & -4.1052019 & -2.0566407 \\ \mathrm{H} & 1.6767471 & -3.2239850 & -2.6856099 \\ \mathrm{C} & 0.5994616 & -3.4002120 & -2.8437658 \\ \mathrm{C} & 0.3647257 & -4.0145520 & -4.2244296 \\ \mathrm{H} & -0.7038758 & -4.2243476 & -4.3880815 \\ \mathrm{H} & 0.6940902 & -3.3324927 & -5.0237554 \\ & 0.9129491 & -4.9599854 & -4.3449953\end{array}$

\section{Compound 5}

46

Energy $=-1089.103620187$ 


\begin{tabular}{|c|c|c|c|}
\hline & -5.2511932 & 2.0292572 & -3.1 \\
\hline $\mathrm{H}$ & -5.7502384 & 0.4593211 & 4329 \\
\hline & -4.8587091 & 1.6130295 & -1.4310960 \\
\hline & -4.9439525 & 1.2061066 & -2.4503783 \\
\hline & -2.7212914 & 2.4448526 & -3.5968990 \\
\hline 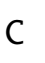 & -3.6205735 & 0.5929780 & 94383 \\
\hline & -3.7237901 & Q. 1756365 & -3.9 \\
\hline & -2.3471636 & 2.0210499 & -1.8970756 \\
\hline & -2.4897926 & 1.6218061 & -2.9082651 \\
\hline c & -1.7085483 & 1.4132427 & -5.5168039 \\
\hline & -3.3364126 & -0.2367690 & -2.2427376 \\
\hline 1 & -1.1983732 & 1.0481872 & -3.3110633 \\
\hline C & -0.8936720 & 1.1135952 & -4.6339910 \\
\hline 0 & 2.7197222 & 0.8250531 & -4.7 \\
\hline 0 & 0. 1989193 & -0.7596136 & -2 . \\
\hline H & 0.1490280 & 1.8520900 & -7.5628113 \\
\hline c & -0.4673282 & 0.2065756 & -2.3194143 \\
\hline $\mathrm{N}$ & 0.4519474 & 0.8290207 & -5.0331352 \\
\hline C & 1.5 & 1.1 & -4.3774575 \\
\hline H & -0.3287472 & -0.3637221 & -6.5419870 \\
\hline c & 0.9576548 & 1.1120238 & -7.4748618 \\
\hline$N$ & -0.6709946 & 0.6079498 & -1.0884491 \\
\hline C & 0.6155088 & 0.1530245 & -6.3355404 \\
\hline $\mathrm{H}$ & 1.8758491 & 1.66 & -7 \\
\hline 0 & 0.4995379 & 3.7237954 & -4.2959322 \\
\hline $\mathrm{H}$ & 0.2316626 & -0.1814478 & -9.0764327 \\
\hline $\mathrm{H}$ & 1.3892298 & 1.0561033 & -9.6113173 \\
\hline 0 & 1.4620331 & 1.9487613 & -3.2280676 \\
\hline 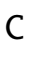 & 1.1459163 & 0.3626447 & -8.7945959 \\
\hline C & 0.7861071 & 3.1641842 & -3.2586135 \\
\hline $\mathrm{H}$ & 1.4041289 & -0.6013659 & -6.21380 \\
\hline $\mathrm{H}$ & -0.5516529 & -0.1342606 & 0.862464 \\
\hline & 0.0189777 & -0.1874925 & -0.0823907 \\
\hline
\end{tabular}




$\begin{array}{lrrr}\text { H } & 0.0738634 & -1.2550489 & -0.3744764 \\ \mathrm{H} & 1.9629575 & -0.3710562 & -8.7213296 \\ \mathrm{H} & -0.0219628 & 4.5720640 & -1.8775848 \\ \mathrm{C} & 0.5312447 & 3.6286065 & -1.8653043 \\ \mathrm{H} & 1.4024278 & 1.3693353 & 0.4848181 \\ \mathrm{C} & 1.4476893 & 0.3106144 & 0.1812670 \\ \mathrm{H} & 2.0013351 & 0.2822636 & -0.7701465 \\ \mathrm{H} & -0.0241021 & 2.8300827 & -1.3396478 \\ \mathrm{H} & 1.4871675 & 3.7579035 & -1.3385948 \\ \mathrm{C} & 2.1733551 & -0.5110927 & 1.2477781 \\ \mathrm{H} & 1.6287324 & -0.4882964 & 2.2048984 \\ \mathrm{H} & 2.2622435 & -1.5653540 & 0.9426951 \\ \mathrm{H} & 3.1882884 & -0.1297941 & 1.4329388\end{array}$

$$
\mathrm{TS}(1+2 \longrightarrow 3)
$$

$\begin{array}{lrrr}20 & & \\ \text { Energy }=-515.5150013488 & \\ \mathrm{H} & -4.5851141 & 0.0177441 & -0.8077599 \\ \mathrm{H} & -2.1972794 & 0.1753358 & -1.5954652 \\ \mathrm{H} & -2.4434286 & 1.1656374 & -0.1521369 \\ \mathrm{C} & -3.7862500 & -0.5353646 & -0.2943823 \\ \mathrm{O} & 3.2841174 & 2.6853542 & -1.6725649 \\ \mathrm{C} & -2.4252927 & 0.1268227 & -0.5189007 \\ \mathrm{H} & -3.7925349 & -1.5673648 & -0.6767125 \\ \mathrm{H} & -4.0381788 & -0.5732980 & 0.7762571 \\ \mathrm{H} & 1.0428923 & 3.8349624 & -1.9728855 \\ \mathrm{C} & 2.2639648 & 2.2906694 & -1.0470650 \\ \mathrm{C} & 0.9415667 & 3.0300386 & -1.2363709 \\ \mathrm{C} & -1.2988640 & -0.6272379 & 0.1884598 \\ \mathrm{~N} & 0.0210421 & -0.0208327 & -0.0993947 \\ \mathrm{C} & 0.5127548 & 0.7439664 & 0.7466935 \\ \mathrm{O} & 0.6405042 & 1.3409225 & 1.7777591\end{array}$




$\begin{array}{rrrr}\mathrm{H} & 0.6128531 & 3.4561908 & -0.2770714 \\ \mathrm{H} & -1.2671316 & -1.6660160 & -0.1747098 \\ \mathrm{O} & 2.2765387 & 1.2869079 & -0.2439637 \\ \mathrm{H} & -1.4959286 & -0.6622440 & 1.2743907 \\ \mathrm{H} & 0.1620083 & 2.3256465 & -1.5580888\end{array}$

$$
\mathrm{TS}(3+2 \longrightarrow 4)
$$

33

Energy $=-802.2840957549$

$\begin{array}{lrrr}\mathrm{O} & -1.1932445 & 2.4712780 & 0.2298468 \\ \mathrm{H} & -5.1395313 & -1.5875255 & 2.5278839 \\ \mathrm{H} & 0.8237010 & 0.8412235 & 0.2583509 \\ \mathrm{O} & -2.6965043 & -1.0969830 & 1.7785591 \\ \mathrm{C} & -2.1213619 & 1.7263480 & 0.1028779 \\ \mathrm{H} & 1.0836384 & -0.8759882 & 0.6588868 \\ \mathrm{C} & -1.2949772 & -1.2619467 & 1.2773267 \\ \mathrm{O} & -0.7397868 & -2.3166362 & 1.6447817 \\ \mathrm{H} & -5.1574011 & -0.1160413 & 1.5415352 \\ \mathrm{H} & -5.0301137 & 2.1320131 & 0.4404939 \\ \mathrm{~N} & -0.9334025 & -0.2095001 & 0.6138639 \\ \mathrm{C} & 0.4266125 & -0.1780714 & 0.1060864 \\ \mathrm{C} & -5.0345147 & -1.2066301 & 1.5027286 \\ \mathrm{C} & -3.6657866 & -1.5335175 & 0.9597376 \\ \mathrm{H} & -4.1501129 & 3.0300701 & -0.8136443 \\ \mathrm{C} & -4.4176921 & 2.0156974 & -0.4704158 \\ \mathrm{~N} & -3.2226793 & 1.2350091 & -0.1232299 \\ \mathrm{H} & 2.2858073 & 0.6469887 & -1.8389534 \\ \mathrm{H} & -5.8088753 & -1.6424315 & 0.8635935 \\ \mathrm{H} & -7.1772934 & 2.1073145 & -0.9606163 \\ \mathrm{H} & 2.5973259 & -1.0588852 & -1.4544963 \\ \mathrm{C} & 0.4865419 & -0.5026342 & -1.3911992 \\ \mathrm{C} & 1.8993059 & -0.3766012 & -1.9640528\end{array}$




$\begin{array}{lrrr}\mathrm{O} & -3.4621404 & -2.1448164 & -0.0820539 \\ \mathrm{H} & -0.2028051 & 0.1739808 & -1.9213897 \\ \mathrm{H} & -6.3426895 & 3.0563615 & -2.2100041 \\ \mathrm{C} & -6.5385968 & 2.0333942 & -1.8540022 \\ \mathrm{C} & -5.2355538 & 1.2970216 & -1.5402298 \\ \mathrm{H} & 0.1010137 & -1.5235691 & -1.5458680 \\ \mathrm{H} & -5.4447538 & 0.2734498 & -1.1932194 \\ \mathrm{H} & 1.9230980 & -0.6139747 & -3.0374239 \\ \mathrm{H} & -4.6221452 & 1.2002066 & -2.4498846 \\ \mathrm{H} & -7.1108765 & 1.5115833 & -2.6335304\end{array}$

$$
\mathrm{TS}(4+2 \longrightarrow 5)
$$

\begin{tabular}{lrrr}
46 & \\
\multicolumn{4}{l}{ Energy $=-1089.072368833$} \\
$\mathrm{H}$ & 6.5557452 & -1.7323785 & 2.4858711 \\
$\mathrm{H}$ & 5.3357064 & -2.5381592 & 0.3153717 \\
$\mathrm{O}$ & 5.3191124 & -1.3211765 & -1.7922546 \\
$\mathrm{C}$ & 5.5806473 & -1.3516869 & 2.8262361 \\
$\mathrm{H}$ & 5.1003594 & -2.1462421 & 3.4177662 \\
$\mathrm{C}$ & 4.4028859 & -2.1225579 & 0.7207319 \\
$\mathrm{H}$ & 3.8803614 & -2.9193363 & 1.2682552 \\
$\mathrm{C}$ & 4.1166908 & -1.4104727 & -1.5674091 \\
$\mathrm{C}$ & 4.7047704 & -0.9400147 & 1.6428014 \\
$\mathrm{~N}$ & 3.5379973 & -1.7495245 & -0.4014643 \\
$\mathrm{H}$ & 5.7679893 & -0.5012777 & 3.4966532 \\
$\mathrm{O}$ & 1.6900877 & -3.0349860 & 0.1734286 \\
$\mathrm{O}$ & 3.1394832 & -1.2730104 & -2.5746050 \\
$\mathrm{H}$ & 5.1990308 & -0.1516947 & 1.0557250 \\
$\mathrm{O}$ & 4.1486858 & 0.4681585 & -3.6905421 \\
$\mathrm{C}$ & 3.1728079 & -0.2111128 & -3.4544039 \\
$\mathrm{H}$ & 1.3943467 & -1.0115845 & -4.3625087 \\
$\mathrm{C}$ & 2.0712990 & -1.8818204 & -0.1830191
\end{tabular}




\begin{tabular}{|c|c|c|c|}
\hline $\mathrm{H}$ & 5.5886930 & 1.2993369 & -1.0504072 \\
\hline C & 1.8108554 & -0.0433430 & -4.0575283 \\
\hline $\mathrm{H}$ & 3.7535330 & -0.5158384 & 1.9993324 \\
\hline $\mathrm{H}$ & 7.2584550 & 3.1477288 & -0.6388087 \\
\hline C & 5.2563036 & 2.2836394 & -0.6872027 \\
\hline $\mathrm{H}$ & 1.8500244 & 0.6471464 & -4.9054457 \\
\hline C & 6.2688202 & 3.3661337 & -1.0641488 \\
\hline $\mathrm{H}$ & 5.1721762 & 2.2093319 & 0.4090610 \\
\hline $\mathrm{N}$ & 1.3973441 & -0.7780818 & -0.3541974 \\
\hline $\mathrm{H}$ & 6.3812651 & 3.4386883 & -2.1565917 \\
\hline $\mathrm{H}$ & 1.1585125 & 0.3614274 & -3.2689177 \\
\hline $\mathrm{N}$ & 2.9328418 & 1.4625339 & -1.0151638 \\
\hline $\mathrm{H}$ & -0.2706221 & -0.9634686 & 0.9353658 \\
\hline C & 3.8733918 & 2.5647767 & -1.2743437 \\
\hline C & -0.0368792 & -0.8796865 & -0.1465089 \\
\hline C & 2.3619476 & 1.3061090 & 0.0614440 \\
\hline $\mathrm{H}$ & -0.4348961 & -1.8056580 & -0.6065094 \\
\hline$H$ & 5.9549615 & 4.3539282 & -0.6936001 \\
\hline 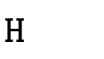 & 3.9542670 & 2.6602765 & -2.3658449 \\
\hline 0 & 1.9157109 & 1.4414706 & 1.1619589 \\
\hline $\mathrm{H}$ & 3.4769581 & 3.5159177 & -0.8785533 \\
\hline C & -0.7775496 & 0. 3292929 & -0.7180054 \\
\hline 4 & -0.3923871 & 1.2438325 & -0.2408034 \\
\hline 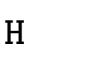 & -0.5408528 & 0.4155195 & -1.7909861 \\
\hline C & -2.2913487 & 0.2342946 & -0.5223368 \\
\hline $\mathrm{H}$ & -2.5464161 & 0. 1268915 & 0.5436407 \\
\hline $\mathrm{H}$ & -2.7069088 & -0.6374367 & -1.0512860 \\
\hline $\mathrm{H}$ & -2.8045977 & 1.1304768 & -0.8996265 \\
\hline TS(5 & $\longrightarrow 6+1)$ & & \\
\hline & & & \\
\hline & & & \\
\hline
\end{tabular}




$\begin{array}{lrrr}\mathrm{H} & -2.8131308 & -4.5653645 & -3.2749468 \\ \mathrm{O} & -2.6675440 & -2.1388440 & -4.2411053 \\ \mathrm{C} & -2.1040480 & -3.9405029 & -2.7230939 \\ \mathrm{H} & -1.0823354 & -4.2930060 & -2.9331660 \\ \mathrm{C} & -2.2098380 & -2.5163593 & -3.1813893 \\ \mathrm{H} & -2.2677298 & -4.0236273 & -1.6425269 \\ \mathrm{H} & -4.3451958 & -0.5343117 & -2.8386897 \\ \mathrm{H} & -6.6774325 & -0.2797459 & -1.9295722 \\ \mathrm{O} & -0.7770793 & -0.0691781 & -3.5759795 \\ \mathrm{O} & -1.7165790 & -1.6704461 & -2.2146851 \\ \mathrm{C} & -4.5414579 & 0.1532997 & -2.0012537 \\ \mathrm{C} & -5.8080063 & -0.2719045 & -1.2579432 \\ \mathrm{H} & 3.1831306 & -3.3952559 & -2.9840649 \\ \mathrm{H} & 0.9624341 & -2.1926834 & -2.7894842 \\ \mathrm{C} & -1.3849722 & -0.3319542 & -2.5572989 \\ \mathrm{H} & -5.6998483 & -1.2826037 & -0.8357918 \\ \mathrm{H} & -4.6706587 & 1.1554678 & -2.4382891 \\ \mathrm{C} & 2.8181197 & -3.0146492 & -2.0188566 \\ \mathrm{H} & 2.3483939 & -3.8554322 & -1.4845604 \\ \mathrm{C} & 1.8319797 & -1.8603709 & -2.2026242 \\ \mathrm{C} & -3.4825048 & 0.9205431 & -0.2777934 \\ \mathrm{C} & -3.3263021 & 0.1828665 & -1.0735743 \\ \mathrm{~N} & -2.8293597 & 0.9496610 & -1.3021289 \\ \mathrm{H} & -2.0857627 & 0.5706061 & -1.7641139 \\ \mathrm{H} & 2.3011368 & -1.0429295 & -2.7715175 \\ \mathrm{H} & -6.0297552 & 0.4153824 & -0.4275641 \\ \mathrm{H} & -3.1706282 & -0.7965465 & -0.6011077 \\ \mathrm{H} & 3.6945137 & -2.6982276 & -1.4314639 \\ \mathrm{C} & 1.3378043 & -1.2968793 & -0.8615516 \\ \mathrm{H} & 0.8725614 & -2.1196306 & -0.2908158 \\ \mathrm{H} & -1.5583087 & 1.8480550 & -1.4966301 \\ \mathrm{H} & -1826672 & -1.2475927\end{array}$




$\begin{array}{lrrr}\mathrm{N} & -0.1966024 & 2.0026437 & -1.5103197 \\ \mathrm{O} & 2.0212497 & 1.3408392 & -1.4333224 \\ \mathrm{H} & 2.2224573 & -0.9608108 & -0.2833509 \\ \mathrm{H} & -0.4369099 & 4.0066820 & -2.0243502 \\ \mathrm{C} & 0.2896085 & 3.3883130 & -1.4818120 \\ \mathrm{H} & 1.2442453 & 3.4010191 & -2.0208026 \\ \mathrm{C} & 0.4858023 & 3.9310211 & -0.0646693 \\ \mathrm{H} & -0.4751871 & 3.8827861 & 0.4699547 \\ \mathrm{H} & 1.1892834 & 3.2719911 & 0.4678069 \\ \mathrm{H} & 0.3193999 & 6.0413306 & -0.5961489 \\ \mathrm{C} & 1.0145765 & 5.3658735 & -0.0741682 \\ \mathrm{H} & 1.9865335 & 5.4263473 & -0.5875462 \\ \mathrm{H} & 1.1503296 & 5.7490208 & 0.9470369\end{array}$

$$
\mathrm{TS}(4 \longrightarrow 24+1)
$$

33

Energy $=-802.2841791178$

$\begin{array}{lrrr}\mathrm{C} & 2.0914550 & 4.8364182 & 2.4153621 \\ \mathrm{C} & 1.0072756 & 3.9481445 & 1.8029311 \\ \mathrm{C} & 1.4691413 & 3.3247634 & 0.4803968 \\ \mathrm{~N} & 0.4671613 & 2.5264796 & -0.2262128 \\ \mathrm{C} & -0.7882865 & 3.0310401 & -0.6367425 \\ \mathrm{O} & -0.9500205 & 4.1968206 & -1.0711138 \\ \mathrm{C} & 0.2060469 & 1.1428556 & 0.0421738 \\ \mathrm{O} & 0.0315335 & 0.9696398 & 1.4776604 \\ \mathrm{C} & -0.5913881 & -0.0948670 & 2.0523395 \\ \mathrm{C} & -1.4309233 & -0.9947622 & 1.1980465 \\ \mathrm{O} & 0.6076586 & 0.2040827 & -0.6311605 \\ \mathrm{O} & -0.4796870 & -0.2159347 & 3.2647316 \\ \mathrm{~N} & -1.6330996 & 1.9988823 & -0.4899070 \\ \mathrm{C} & -2.8241312 & 1.9312800 & -1.3164144 \\ \mathrm{C} & -3.9929020 & 1.2203356 & -0.6330261\end{array}$




$\begin{array}{lrrr}\mathrm{C} & -5.2668702 & 1.2460203 & -1.4793952 \\ \mathrm{H} & 2.3535187 & 2.6893575 & 0.6388372 \\ \mathrm{H} & 1.7763306 & 4.1274340 & -0.2096782 \\ \mathrm{H} & 3.0002164 & 4.2564705 & 2.6385550 \\ \mathrm{H} & 2.3751291 & 5.6486195 & 1.7284019 \\ \mathrm{H} & -0.9569143 & -1.1981840 & 0.2333783 \\ \mathrm{H} & -2.6057189 & 1.4074780 & -2.2711482 \\ \mathrm{H} & -1.6378427 & -1.9197371 & 1.7449650 \\ \mathrm{H} & -3.7103707 & 0.1760557 & -0.4262652 \\ \mathrm{H} & 0.7273532 & 3.1486628 & 2.5029937 \\ \mathrm{H} & -5.0998239 & 0.7745075 & -2.4603139 \\ \mathrm{H} & -2.3782132 & -0.4735336 & 0.9966394 \\ \mathrm{H} & 1.7488236 & 5.2952865 & 3.3535177 \\ \mathrm{H} & 0.0986058 & 4.5399048 & 1.6091545 \\ \mathrm{H} & -3.1358883 & 2.9564086 & -1.5948804 \\ \mathrm{H} & -6.0889332 & 0.7081693 & -0.9860786 \\ \mathrm{H} & -4.1748663 & 1.6967030 & 0.3444673 \\ \mathrm{H} & -5.6013376 & 2.2791673 & -1.6601561\end{array}$

\subsubsection{Pre-Catalyst Activation}

\section{Compound 7}

\begin{tabular}{llrr}
33 & & \\
\multicolumn{4}{l}{ Energy $=-802.3477128515$} \\
$\mathrm{H}$ & 6.6023303 & -2.5361684 & 2.5138853 \\
$\mathrm{O}$ & 5.0581437 & -0.3330438 & -0.8601209 \\
$\mathrm{H}$ & 5.2606802 & -2.3106150 & 0.3014679 \\
$\mathrm{C}$ & 5.6426694 & -2.5131276 & 3.0525303 \\
$\mathrm{H}$ & 5.8247747 & -2.0759583 & 4.0442686 \\
$\mathrm{H}$ & 4.9310272 & -0.6694272 & 2.1590150 \\
$\mathrm{C}$ & 3.8418083 & -0.2943471 & -0.5554374 \\
$\mathrm{C}$ & 4.3355853 & -2.3058384 & 0.8908867 \\
$\mathrm{H}$ & 5.3169048 & -3.5542652 & 3.1989930
\end{tabular}




$\begin{array}{rrrr}\mathrm{C} & 4.5957881 & -1.7124607 & 2.2771555 \\ \mathrm{O} & 2.4054672 & -1.7571599 & -2.5886499 \\ \mathrm{H} & 3.9633969 & -3.3340520 & 0.9846505 \\ \mathrm{H} & 0.1125682 & 0.4742356 & -2.4143073 \\ \mathrm{O} & 3.0193743 & 0.6359850 & -0.6916158 \\ \mathrm{~N} & 3.3489029 & -1.5352487 & 0.1242581 \\ \mathrm{C} & 1.4062324 & -1.2434383 & -2.0700448 \\ \mathrm{H} & 3.6467809 & -1.6838717 & 2.8348127 \\ \mathrm{C} & 0.4133654 & -0.4616891 & -2.9015285 \\ \mathrm{H} & -0.4942832 & -1.0556365 & -3.0843066 \\ \mathrm{C} & 2.0577724 & -1.9517441 & 0.1939307 \\ \mathrm{H} & 0.8813253 & -0.2421356 & -3.8665289 \\ \mathrm{O} & 1.6588009 & -2.7962815 & 1.0113024 \\ \mathrm{~N} & 1.1186791 & -1.3871820 & -0.7287954 \\ \mathrm{C} & -0.1731133 & -0.9940276 & -0.1432761 \\ \mathrm{H} & -0.9333221 & -1.0009674 & -0.9320086 \\ \mathrm{H} & -0.4492848 & -1.7798639 & 0.5726203 \\ \mathrm{H} & \mathbb{0} .1913261 & 1.1347308 & -0.1608984 \\ \mathrm{H} & \mathbb{0} .6510710 & 0.3357277 & 1.3364509 \\ \mathrm{C} & -0.1261628 & 0.3643745 & 0.5570766 \\ \mathrm{C} & -1.4812199 & 0.7217341 & 1.1682269 \\ \mathrm{H} & -2.2626346 & 0.7774965 & 0.3949979 \\ \mathrm{H} & -1.7951246 & -0.0320053 & 1.9062306 \\ \mathrm{H} & -1.4426907 & 1.6949716 & 1.6767351 \\ & & & \\ & & & \\ \mathrm{H} & & \end{array}$

\subsubsection{Deprotonated Amide Catalysis}

\section{Compound 12}
17
Energy $=-326.8302359678$

$\begin{array}{rrrr}\mathrm{H} & 0.1634436 & 1.7805573 & 1.2496916 \\ \mathrm{O} & -0.1937100 & 1.6147208 & 3.9089797 \\ \mathrm{H} & 1.0185585 & 0.3649283 & 1.8478187\end{array}$




$\begin{array}{lrrr}\text { C } & 0.0691914 & 0.6790531 & 1.3628471 \\ \text { H } & -2.8080450 & 1.4155198 & 4.5802138 \\ \text { C } & -1.0872062 & 0.8479015 & 3.3925940 \\ \text { H } & -0.9587864 & 0.3647782 & -0.5024273 \\ \text { N } & -1.0860356 & 0.3253938 & 2.1777554 \\ \text { C } & -2.2870668 & 0.4956172 & 4.2706923 \\ \text { C } & -0.0195585 & 0.0397352 & -0.0230640 \\ \text { H } & -0.0994179 & -1.0542761 & 0.0968217 \\ \text { H } & -1.9446576 & -0.0030334 & 5.1913209 \\ \text { H } & -2.9986810 & -0.1606035 & 3.7526040 \\ \text { H } & 1.2519306 & 1.4690378 & -1.0750797 \\ \mathrm{C} & 1.1710934 & 0.3815958 & -0.9207803 \\ \text { H } & 2.1170650 & 0.0406520 & -0.4715866 \\ \text { H } & 1.0825537 & -0.0901136 & -1.9103538\end{array}$

\section{Compound $12+\mathrm{H}^{+}$}

18

Energy $=-327.3282306066$

$\begin{array}{lrrr}\text { C } & -2.3417258 & 0.4474555 & 4.0840354 \\ \mathrm{C} & -1.2926191 & 0.9574020 & 3.1230298 \\ \mathrm{~N} & -0.6506553 & 0.0160655 & 2.4041891 \\ \mathrm{C} & 0.3602644 & 0.3005913 & 1.3943442 \\ \mathrm{C} & -0.1170790 & -0.0382260 & -0.0191570 \\ \mathrm{C} & -1.3120890 & 0.7998663 & -0.4675391 \\ \mathrm{O} & -1.0496768 & 2.1675380 & 3.0195627 \\ \mathrm{H} & 0.5931153 & 1.3624049 & 1.4780991 \\ \mathrm{H} & 1.2612395 & -0.2703763 & 1.6336373 \\ \mathrm{H} & -3.3035396 & 0.8917381 & 3.8193355 \\ \mathrm{H} & -0.3684716 & -1.1035445 & -0.0650638 \\ \mathrm{H} & 0.7261263 & 0.1150028 & -0.7001564 \\ \mathrm{H} & -2.0868281 & 0.7799639 & 5.0922534 \\ \mathrm{H} & -2.4342405 & -0.6389761 & 4.0756542\end{array}$




$\begin{array}{lrrr}\mathrm{H} & -2.1624543 & 0.6603186 & 0.2051236 \\ \mathrm{H} & -1.0608862 & 1.8641462 & -0.4753687 \\ \mathrm{H} & -1.6305423 & 0.5176857 & -1.4739115 \\ \mathrm{H} & -0.9181809 & -0.9471758 & 2.5437175\end{array}$

\section{Compound 8}

\begin{tabular}{|c|c|c|c|}
\hline \multicolumn{4}{|c|}{ Energy $=-613.6308423646$} \\
\hline 0 & -0.4994749 & -2.3084753 & 2.4014508 \\
\hline & -2.5422264 & -1.9236576 & 0.9882634 \\
\hline $\mathrm{H}$ & 1.3025417 & -0.9119002 & 3.0465339 \\
\hline C & -0.4797625 & -1.5842578 & 1.3785477 \\
\hline & -1.6626686 & -1.5774475 & 0.4320121 \\
\hline & 3.7321524 & -1.6592834 & 3.6712316 \\
\hline $\mathrm{H}$ & -1.4729707 & -2.2597227 & -0.4066019 \\
\hline $\mathrm{H}$ & 2.4875289 & -2.6305141 & 1.6868980 \\
\hline $\mathrm{H}$ & -1.8510140 & -0.5869859 & 0.0011812 \\
\hline C & 1.6801233 & -0.6660517 & 2.0450724 \\
\hline $\mathrm{N}$ & 0.5666875 & -0.7672962 & 1.1016760 \\
\hline C & 4.0261500 & -1.4260865 & 2.6363594 \\
\hline C & 2.8455499 & -1.5888200 & 1.6782418 \\
\hline $\mathrm{H}$ & 4.8563790 & -2.0933317 & 2.3652233 \\
\hline $\mathrm{H}$ & 2.0075916 & 0.3837797 & 2.0409218 \\
\hline $\mathrm{H}$ & 4.4068054 & -0.3932673 & 2.6228839 \\
\hline $\mathrm{H}$ & 3.1601043 & -1.3704142 & 0.6452311 \\
\hline C & 0.6826484 & 0.0441106 & -0.1304338 \\
\hline $\mathrm{N}$ & 0.6382628 & -0.6590040 & -1.2320677 \\
\hline 0 & 0.8356510 & 1.2915972 & 0.0517836 \\
\hline $\mathrm{H}$ & -0.3124403 & -1.3420329 & -3.6456453 \\
\hline 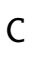 & 0.6965962 & 0.1359127 & -2.4508713 \\
\hline & -0.1286929 & 0.8782115 & -2.4844082 \\
\hline $\mathrm{H}$ & 1.4458764 & -1.4862421 & -3.6498483 \\
\hline
\end{tabular}




$\begin{array}{rrrr}\mathrm{C} & 0.6204724 & -0.7554340 & -3.6897944 \\ \mathrm{H} & 1.6276608 & 0.7398541 & -2.4928298 \\ \mathrm{C} & 0.6829422 & 0.0337259 & -4.9985032 \\ \mathrm{H} & -0.1494257 & 0.7512718 & -5.0691957 \\ \mathrm{H} & 0.6274806 & -0.6291130 & -5.8741571 \\ \mathrm{H} & 1.6204913 & 0.6067444 & -5.0718452\end{array}$

\section{Compound $8+\mathrm{H}^{+}$}

31

Energy $=-614.1165261391$

$\begin{array}{lrrr}\mathrm{C} & -4.2678575 & -1.5567331 & -3.5568944 \\ \mathrm{C} & -3.1723806 & -1.1803629 & -2.5905633 \\ \mathrm{~N} & -2.1140622 & -0.4485114 & -3.0832401 \\ \mathrm{C} & -0.9974761 & 0.0111534 & -2.3087166 \\ \mathrm{O} & -0.1130230 & 0.6618270 & -2.8668789 \\ \mathrm{O} & -3.2484755 & -1.5416330 & -1.4110875 \\ \mathrm{C} & -2.0854971 & -0.0303449 & -4.5000498 \\ \mathrm{C} & -2.6864927 & 1.3554038 & -4.7177802 \\ \mathrm{C} & -2.6233376 & 1.7601957 & -6.1890164 \\ \mathrm{~N} & -0.9974156 & -0.2927350 & -1.0054465 \\ \mathrm{C} & 0.1127946 & 0.0739804 & -0.1375132 \\ \mathrm{C} & 1.2456147 & -0.9533935 & -0.1517963 \\ \mathrm{C} & 2.3907783 & -0.5416449 & 0.7710092 \\ \mathrm{H} & -5.0722412 & -2.0099777 & -2.9823390 \\ \mathrm{H} & -2.6100129 & -0.7811196 & -5.0864824 \\ \mathrm{H} & -3.1788694 & 1.0550604 & -6.8140975 \\ \mathrm{H} & -3.8990000 & -2.2809355 & -4.2875499 \\ \mathrm{H} & -3.7248301 & 1.3635669 & -4.3716640 \\ \mathrm{H} & -4.6495265 & -0.6927259 & -4.1021544 \\ \mathrm{H} & -3.0520161 & 2.7535371 & -6.3379971 \\ \mathrm{H} & -1.0422623 & -0.0371022 & -4.8076600 \\ \mathrm{H} & -1.5889850 & 1.7814023 & -6.5439277\end{array}$




$\begin{array}{rrrr}\mathrm{H} & -2.1375081 & 2.0745744 & -4.1044016 \\ \mathrm{H} & 1.6084959 & -1.0609437 & -1.1782940 \\ \mathrm{H} & 0.4869752 & 1.0484308 & -0.4567536 \\ \mathrm{H} & 0.8466218 & -1.9259249 & 0.1534914 \\ \mathrm{H} & -0.2895418 & 0.1785431 & 0.8722837 \\ \mathrm{H} & 2.8133983 & 0.4197742 & 0.4646093 \\ \mathrm{H} & 3.1930195 & -1.2828715 & 0.7545233 \\ \mathrm{H} & 2.0450604 & -0.4420275 & 1.8042098 \\ \mathrm{H} & -1.7669221 & -0.8694676 & -0.6741472\end{array}$

\section{Compound 9}

$\begin{array}{lrrr}\text { 43 } & & \\ \text { Energy }=-900.4249093604 & \\ \text { H } & -4.3225954 & -4.3616710 & -2.8871381 \\ \text { H } & -3.7434379 & -4.5870744 & -1.2215984 \\ \text { C } & -3.8078339 & -3.8674762 & -2.0517383 \\ \text { O } & -2.4403996 & 0.7254194 & -0.5896364 \\ \text { H } & -1.3093903 & 1.1383776 & 1.6016712 \\ \text { H } & -1.8058733 & -4.2247803 & -2.8212588 \\ \text { H } & -2.0051104 & -0.0810939 & 3.7983950 \\ \text { H } & -4.4362706 & -3.0277398 & -1.7181781 \\ \text { H } & 0.3823830 & 0.7015557 & 1.9495437 \\ \text { H } & -0.3118054 & -0.5292801 & 4.0919248 \\ \text { C } & -2.4186959 & -3.3809119 & -2.4670271 \\ \text { H } & -2.0727155 & 1.6198258 & -2.8816384 \\ \text { C } & -0.5320276 & 0.3672118 & 1.4281381 \\ \text { C } & -1.2336892 & -0.8334907 & 3.5717155 \\ \text { C } & -1.2456681 & 0.3521620 & -0.7875589 \\ \text { H } & -2.5060724 & -2.6771719 & -3.3106598 \\ \text { H } & -1.1653662 & 3.8149900 & -3.9359895 \\ \text { H } & -1.5354646 & -3.3968660 & -0.4796113 \\ \text { H } & -1.5619023 & -1.7892726 & 4.0058947\end{array}$




\begin{tabular}{|c|c|c|c|}
\hline & -1.1376279 & 1.1224855 & -3.1773086 \\
\hline & -0.2222964 & 0.2095080 & 0.0124968 \\
\hline & -1.0013525 & -0.9495955 & 2.0643570 \\
\hline & -1.6998404 & -2.6942389 & -1.3054916 \\
\hline & -1.2800686 & 0.6677078 & -4.1669413 \\
\hline & -0.9125202 & 0.0494182 & -2.2090173 \\
\hline & -1.9283311 & -1.2667861 & 1.5604097 \\
\hline & -2.3056745 & -1.8574764 & -0.9256056 \\
\hline & -0.2527632 & 3.2620237 & -4.2059450 \\
\hline & -0.3875059 & 2.8740545 & 72236 \\
\hline & Q. 1693546 & 2.5321905 & -2.2040118 \\
\hline & -0.2512688 & -1.7278140 & 1.8493134 \\
\hline & 0.0159306 & 2.1274305 & -3.2168213 \\
\hline C & -0.3238801 & -1.0902130 & -2.6209004 \\
\hline & -0.3753632 & -2.1846909 & -1.6778323 \\
\hline & 0.5804037 & 3.9783115 & -4.2264803 \\
\hline & 0.1616707 & -1.2625386 & -3.7490381 \\
\hline & 0.9390932 & 1.5925316 & -3.4888989 \\
\hline C & 0.7604627 & -2.6982122 & -1.1145690 \\
\hline 0 & 0.7264602 & -3.6822998 & -0.3556704 \\
\hline & 1.9152892 & -0.9105402 & -1.1231835 \\
\hline & 2.0449592 & -1.9639145 & -1.4157863 \\
\hline & 2.2851914 & -1.9994341 & -2.4856809 \\
\hline & 2.8566461 & -2.4179987 & -0.8385921 \\
\hline
\end{tabular}

\section{Compound $9+\mathrm{H}^{+}$}

44

Energy $=-900.8942991194$

$\begin{array}{llll}\text { C } & -3.5635761 & -3.7992462 & -1.8198487 \\ \text { C } & -2.2048136 & -3.3112204 & -2.3178306 \\ \text { C } & -1.4573692 & -2.5625745 & -1.2192155 \\ \text { N } & -0.1374524 & -2.0766284 & -1.6782773\end{array}$




\begin{tabular}{|c|c|c|c|}
\hline & 0.9449481 & -2.9401614 & -1.5610459 \\
\hline & 2.3037906 & -2.4568524 & 50210 \\
\hline & -0.0852678 & -0.9477646 & -2.5324362 \\
\hline & 0.5067159 & -0.9748578 & -3.5997650 \\
\hline & -0.8067966 & Q. 1492345 & 45504 \\
\hline & -1.1363124 & 1.1582983 & 13005 \\
\hline & -0.1165187 & 2.2915560 & -3.1972702 \\
\hline & -0.4847334 & 3.3258320 & -4.2589083 \\
\hline & -1.1672323 & 663 & 44414 \\
\hline & 77446 & 8936 & 05696 \\
\hline & -0.6251578 & 0.2660068 & 1.5988323 \\
\hline & -1.4183899 & -0.8978502 & 2.1925163 \\
\hline & -1.6944516 & -0.6846514 & 3.6792519 \\
\hline & -2.1640942 & 1.1820697 & -0.5 \\
\hline & 0.7888075 & -4.0454496 & 58852 \\
\hline & -4.0978305 & -4.3308170 & -2.6099189 \\
\hline & -3.4476877 & -4.4798774 & -0.9718309 \\
\hline & .1801163 & 1. & 1.7040747 \\
\hline & -1.5962903 & -4 & -2.6518888 \\
\hline & -2.2731751 & 0.2289596 & 3.8423025 \\
\hline & -4.1863915 & -2.9600603 & -1.4966460 \\
\hline & 0.3307151 & 0. 3777692 & 2.1134040 \\
\hline & -0.7604814 & -0.5964663 & 4.2416864 \\
\hline & -2.1280739 & 1.5369338 & -2.9082222 \\
\hline & -2.3393083 & -2.6468828 & -3.1776620 \\
\hline & -1.4654373 & 3.7648880 & -4.0545814 \\
\hline & -1.2725960 & -3.2081281 & -0.3631324 \\
\hline & -2.2604168 & -1.5216541 & 4.093 \\
\hline & -1.1790465 & 0.6362875 & -4.1055177 \\
\hline & -2.3614860 & -0.9993273 & 1.6470905 \\
\hline & -2.0422972 & -1.7092938 & -0.8814 \\
\hline & -0.5199059 & 2.8706813 & -5.2528268 \\
\hline & -0.0676737 & 2.7687859 & -2.2132303 \\
\hline
\end{tabular}




$\begin{array}{rrrr}\text { H } & -0.8574129 & -1.8257473 & 2.0444185 \\ \mathrm{H} & 0.2487699 & 4.1345680 & -4.2839048 \\ \mathrm{H} & 0.8716375 & 1.8716603 & -3.4044920 \\ \mathrm{H} & 2.4235894 & -1.3778555 & -1.9057112 \\ \mathrm{H} & 2.4645811 & -2.7264036 & -3.0403315 \\ \mathrm{H} & 3.0431097 & -2.9691988 & -1.3811543 \\ \mathrm{H} & 0.4805982 & -0.4583852 & -0.0689789\end{array}$

\section{Compound 10}

$\begin{array}{lrrr}\text { S6 } & & \\ \text { Energy }=-1187.220216556 & \\ \mathrm{H} & 2.6070673 & -2.3187130 & -6.1743287 \\ \mathrm{H} & 3.7326350 & -2.9701726 & -4.9626826 \\ \mathrm{H} & -2.0980487 & 2.1959645 & -2.6313656 \\ \mathrm{H} & -4.2232121 & 3.2019173 & -3.5473571 \\ \mathrm{C} & 3.4114151 & -2.0496301 & -5.4730199 \\ \mathrm{H} & 4.2608397 & -1.6773127 & -6.0619739 \\ \mathrm{O} & -1.7004443 & -1.6078537 & -4.9202008 \\ \mathrm{H} & -2.8131075 & -3.2205636 & -2.5654401 \\ \mathrm{H} & 0.9141361 & -1.7724201 & -4.3104766 \\ \mathrm{H} & -2.6832716 & -5.4115194 & -1.2034139 \\ \mathrm{H} & -3.2088346 & 0.9281576 & -3.1611710 \\ \mathrm{C} & -2.5835967 & 1.7616605 & -3.5187776 \\ \mathrm{H} & -1.3860582 & -3.7082359 & -3.4842931 \\ \mathrm{C} & -3.4471816 & 2.8103255 & -4.2198666 \\ \mathrm{H} & -2.8383729 & 3.6614440 & -4.5614039 \\ \mathrm{H} & 2.0233333 & -2.4096043 & -3.0830968 \\ \mathrm{H} & -1.2341212 & -5.8964211 & -2.1079089 \\ \mathrm{C} & 1.7487698 & -1.5107939 & -3.6490526 \\ \mathrm{C} & -1.7047107 & -3.1548856 & -2.5767069 \\ \mathrm{C} & -1.2814017 & -1.2095000 & -3.7919732 \\ \mathrm{C} & 2.9360052 & -1.0010303 & -4.4668700\end{array}$




\begin{tabular}{|c|c|c|c|}
\hline & 2.6396151 & -0.0794656 & -4.9 \\
\hline & -1.5861393 & -5 & 2728 \\
\hline & -3.9496504 & 2.3843419 & -5.1016827 \\
\hline & -0.0783182 & -0.2506144 & 1.8856833 \\
\hline & 3.6588501 & 2.4616370 & 0.2400018 \\
\hline & -1.2687980 & -1.7710563 & -2.6174487 \\
\hline & -0.6996503 & Q. 1647462 & -3.7936927 \\
\hline & -1.5013972 & 1.2024265 & -4.4455272 \\
\hline & -1.1572258 & -3.8566926 & 29573 \\
\hline & -1.1838441 & -5.8026703 & 1595 \\
\hline & 3.7528685 & -0.7303243 & -3.7807220 \\
\hline & -0.8275647 & 2.0045607 & -4.7744722 \\
\hline & 1.2750984 & -0.5138286 & -2.6780396 \\
\hline & -1.4909545 & -3.3033342 & -0.4408041 \\
\hline & 0.4085398 & 0.5527439 & 38487 \\
\hline & -0.1162719 & -1.6773484 & 0.8331008 \\
\hline & -2.2997031 & -1.4741454 & 2.0492588 \\
\hline & 3.5 & 7 & 196 \\
\hline 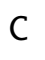 & -0.5 & -( & 0.99 \\
\hline & 3.3520639 & 1.4766706 & -0.1276067 \\
\hline & -1.9546358 & 0.7348246 & -5.3300531 \\
\hline & -0.0574411 & -3.7801421 & -1.3461544 \\
\hline & 0.7413348 & 1.7315273 & -2.9451901 \\
\hline & -2.0676867 & -0.8107375 & 1.2044049 \\
\hline $\mathrm{N}$ & 1.1844544 & 0.3330336 & -0.4751209 \\
\hline ( & 1.8949797 & 1.2798721 & 0.2233026 \\
\hline 4 & 1.8241996 & -0.5180921 & -1.4307792 \\
\hline & -2.5241736 & 0.1696388 & 1.4095621 \\
\hline$f$ & 3.9652585 & 0.7065162 & 0.3589577 \\
\hline 0 & 1.3380389 & 1.9738452 & 1.0872166 \\
\hline c & -0.2 & 0.1847252 & -0.230 \\
\hline I & -0.6874007 & 1.1898461 & -0.1104102 \\
\hline t & -2.5471581 & -1.2213153 & 0.3031936 \\
\hline
\end{tabular}




$\begin{array}{lrrr}0 & 2.7761270 & -1.2373324 & -1.1151734 \\ \mathrm{H} & -0.7096400 & -0.3097660 & -1.1080252\end{array}$

\section{Compound 11}

\begin{tabular}{lrrr} 
56 & \multicolumn{4}{l}{} \\
Energy $=-1187.240425626$ & \\
$\mathrm{H}$ & 1.0583936 & 1.8698577 & 3.2160223 \\
$\mathrm{H}$ & 6.2539619 & 0.1807693 & 0.5367739 \\
$\mathrm{H}$ & 0.8322819 & 0.4916225 & 2.1052934 \\
$\mathrm{C}$ & 0.6972851 & 1.5733999 & 2.2256129 \\
$\mathrm{O}$ & -1.3458104 & 2.3387689 & 3.1859928 \\
$\mathrm{H}$ & 3.7677040 & -0.1791576 & 0.7323197 \\
$\mathrm{H}$ & 1.2969207 & 2.0677562 & 1.4546571 \\
$\mathrm{H}$ & 4.1231435 & 1.5118397 & 0.3450857 \\
$\mathrm{C}$ & -0.7680460 & 1.9486154 & 2.1434175 \\
$\mathrm{C}$ & 5.6223530 & 0.1086124 & -0.3595541 \\
$\mathrm{C}$ & 4.1735531 & 0.4841393 & -0.0472842 \\
$\mathrm{H}$ & 5.6913357 & -0.9227473 & -0.7375578 \\
$\mathrm{H}$ & -0.1355601 & 4.3461420 & 1.2415705 \\
$\mathrm{H}$ & -2.1653144 & -0.6037572 & -0.1404234 \\
$\mathrm{H}$ & 1.3846639 & 4.6563249 & 0.3887360 \\
$\mathrm{H}$ & 6.0480647 & 0.7745629 & -1.1255134 \\
$\mathrm{H}$ & 0.1722029 & 6.8116592 & 0.8848074 \\
$\mathrm{O}$ & 1.4393950 & -1.4545779 & -0.5000460 \\
$\mathrm{H}$ & -3.4812354 & 0.9332592 & 2.5267503 \\
$\mathrm{C}$ & 0.2897090 & 4.7132067 & 0.2957195 \\
$\mathrm{H}$ & -0.9662264 & -1.8351610 & -0.5735390 \\
$\mathrm{~N}$ & -1.4415504 & 1.8600026 & 0.9632353 \\
$\mathrm{C}$ & 1.0336751 & -0.2781685 & -0.5549454 \\
$\mathrm{C}$ & -1.1702988 & -0.9952772 & 0.1062586 \\
$\mathrm{H}$ & -2.9157359 & 3.2056820 & 1.5701460 \\
$\mathrm{C}$ & 3.2820136 & 0.3871647 & -1.2862865 \\
& & & \\
& &
\end{tabular}




$\begin{array}{lrrr}\mathrm{N} & -0.2228219 & 0.0823293 & -0.1953202 \\ \mathrm{C} & -0.1454335 & 6.1599044 & 0.0590323 \\ \mathrm{~N} & 1.8864491 & 0.7401371 & -0.9987487 \\ \mathrm{H} & -3.1522812 & -2.3000477 & 1.5563809 \\ \mathrm{H} & 3.2837872 & -0.6355059 & -1.6802939 \\ \mathrm{C} & 1.5213430 & 2.0893387 & -1.0936293 \\ \mathrm{~N} & 0.2655449 & 2.4088325 & -0.6994110 \\ \mathrm{C} & -2.8416698 & 2.2860016 & 0.9706257 \\ \mathrm{H} & -1.2404092 & 6.2351803 & -0.0263482 \\ \mathrm{C} & -3.8122488 & 1.2381297 & 1.5231379 \\ \mathrm{C} & -0.8527987 & 1.4301320 & -0.4335890 \\ \mathrm{O} & 2.3386689 & 2.9345345 & -1.5051548 \\ \mathrm{H} & 3.6349380 & 1.0707870 & -2.0669317 \\ \mathrm{H} & -5.3115506 & 2.6526695 & 2.2374458 \\ \mathrm{H} & 0.2896495 & 6.5578719 & -0.8704523 \\ \mathrm{H} & -1.9016996 & -3.4847303 & 1.1203515 \\ \mathrm{C} & -0.1591194 & 3.8029782 & -0.8493766 \\ \mathrm{C} & -2.1243442 & -2.6281064 & 1.7747361 \\ \mathrm{H} & -3.7728073 & 0.3428582 & \mathbb{0} .8838992 \\ \mathrm{C} & -1.1275588 & -1.4902793 & 1.5523387 \\ \mathrm{C} & -5.2436602 & 1.7729560 & 1.5789775 \\ \mathrm{H} & -1.2539196 & 3.7887686 & -0.9267834 \\ \mathrm{H} & -5.9409776 & 1.0137922 & 1.9607395 \\ \mathrm{H} & -1.3596961 & -0.6561785 & 2.2301937 \\ \mathrm{H} & -3.0986144 & 2.5091380 & -0.0714664 \\ \mathrm{H} & 0.2344122 & 4.1889984 & -1.8012291 \\ \mathrm{H} & -1.7585240 & 1.4277820 & -1.3382056 \\ \mathrm{H} & -2.0993184 & -2.9829965 & 2.8143871 \\ -0.1066504 & -1.8278937 & 1.7872497 \\ & -5.5926155 & 2.0774010 & 0.5800746\end{array}$




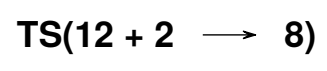


$\mathrm{H} \quad 2.9710396 \quad-1.1279227 \quad-6.6753552$

$\mathrm{TS}(8+2 \longrightarrow 9)$

43

Energy $=-900 \cdot 3972085343$

$\mathrm{H} \quad-3.7788316 \quad-4.3013689 \quad-0.0745378$

$\mathrm{H} \quad-2.5265948 \quad-5.3950478 \quad 0.5528312$

$\begin{array}{llll}\text { C } & -2.8126120 & -4.3365992 & 0.4493623\end{array}$

$\mathrm{H} \quad-2.9671740 \quad-3.9353610 \quad 1.4633880$

$\begin{array}{llll}\text { C } & -1.7383063 & -3.5416996 & -0.2933461\end{array}$

$\mathrm{H} \quad-1.6034487-3.9407477 \quad-1.3123578$

$\mathrm{H} \quad-2.0571814 \quad-2.4934292 \quad-0.4070133$

$\begin{array}{llll}\mathrm{H} & -3.0156332 & 0.1765669 & 0.6010295\end{array}$

$\mathrm{H} \quad-0.0301812 \quad-4.5989012 \quad 0.5362872$

$\begin{array}{llll}\text { C } & -0.3892980 & -3.5578235 & 0.4267228\end{array}$

$\mathrm{H} \quad-2.8819500 \quad-0.7678163 \quad 2.0878966$

$\mathrm{H} \quad-4.5821969 \quad 1.0730358 \quad 2.3559990$

$\begin{array}{llll}\text { C } & -2.6979258 & 0.2278007 & 1.6545844\end{array}$

$\begin{array}{llll}0 & 0.3040067 & -0.0856828 & -1.1876598\end{array}$

$\mathrm{H} \quad-0.5384711-3.1683100 \quad 1.4538626$

$\begin{array}{llll}\text { C } & -0.0179568 & -0.4757208 & -0.1067980\end{array}$

$\begin{array}{llll}\text { C } & -3.5061496 & 1.2916955 & 2.3994847\end{array}$

$\mathrm{H} \quad \begin{array}{llll}1.4459175 & -3.6296562 & -2.7117374\end{array}$

$\begin{array}{llll}\mathrm{N} & -0.4292230 & -0.5633669 & 1.0501798\end{array}$

$\begin{array}{llll}\mathrm{N} & 0.5806658 & -2.7359839 & -0.2690964\end{array}$

$\mathrm{H} \quad-3.3484115 \quad 2.2895808 \quad 1.9627984$

$\mathrm{H} \quad \begin{array}{llll}0.6227568 & -2.0767984 & -2.8429157\end{array}$

$\begin{array}{llll}\text { C } & -1.1961429 & 0.5117308 & 1.7031556\end{array}$

$\begin{array}{llll}\text { C } & 1.5830174 & -2.5815233 & -3.0031581\end{array}$

$\mathrm{H} \quad 1.8676677 \quad-2.5139571 \quad-4.0601810$

$\begin{array}{llll}\mathrm{H} & -0.9817328 & 1.4910937 & 1.2413654\end{array}$

$\mathrm{H} \quad-3.2152499 \quad 1.3401624 \quad 3.4596850$ 


$\begin{array}{rrrr}\mathrm{C} & 1.8417799 & -3.0622309 & -0.1234112 \\ \mathrm{H} & -0.8649747 & 0.5614423 & 2.7515825 \\ \mathrm{O} & 2.3788173 & -3.9774935 & 0.5695579 \\ \mathrm{C} & 2.6548456 & -1.8894154 & -2.1892883 \\ \mathrm{~N} & 2.7716424 & -2.1972720 & -0.8692855 \\ \mathrm{O} & 3.4373330 & -1.0835847 & -2.7411573 \\ \mathrm{H} & 2.5530812 & -0.5770566 & 1.2875465 \\ \mathrm{C} & 3.8582425 & -1.6040999 & -0.0872141 \\ \mathrm{H} & 4.1923388 & -2.3687072 & 0.6281190 \\ \mathrm{H} & 3.0734661 & 0.4007322 & -0.0860123 \\ \mathrm{C} & 3.4209024 & -0.3372674 & 0.6528724 \\ \mathrm{H} & 4.6843097 & -1.3765795 & -0.7730193 \\ \mathrm{C} & 4.5503801 & 0.2468941 & 1.5011099 \\ \mathrm{H} & 4.8941673 & -0.4767502 & 2.2563192 \\ \mathrm{H} & 4.2250825 & 1.1539405 & 2.0301049 \\ \mathrm{H} & 5.4173956 & 0.5147353 & 0.8778150\end{array}$

$$
\mathrm{TS}(9+2 \longrightarrow 10)
$$

\begin{tabular}{|c|c|c|c|}
\hline \multicolumn{4}{|c|}{ Energy $=-1187.202478990$} \\
\hline $\mathrm{H}$ & -3.5456743 & -5.3431047 & -2.0017860 \\
\hline C & -2.9094670 & -5.4091728 & -1.1053690 \\
\hline & -3.4774768 & -5.9451686 & -0.3311917 \\
\hline & -2.0339036 & -6.0243206 & -1.3647361 \\
\hline $\mathrm{H}$ & -2.3323966 & -3.1466384 & -2.6014054 \\
\hline 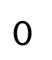 & -3.3871366 & -0.9062614 & -2.7837234 \\
\hline C & -1.7047204 & -3.2409662 & -1.6997440 \\
\hline$C$ & -2.4844588 & -4.0190038 & -0.6314325 \\
\hline & -0.3601820 & 0.1706726 & -4.3348136 \\
\hline & -0.8038359 & -3.8182129 & -1.9756351 \\
\hline & -3.3718426 & -3.4299134 & -0.3457262 \\
\hline & -3.2352184 & -0.6978400 & -1.6133550 \\
\hline
\end{tabular}




$\begin{array}{lrrr}\mathrm{H} & -2.1642599 & 1.3490322 & -3.3277658 \\ \mathrm{H} & 1.9678038 & -0.5903558 & -3.8491281 \\ \mathrm{C} & 0.0908691 & 0.1022294 & -3.1853023 \\ \mathrm{H} & -2.6353553 & 3.7178915 & -4.2541752 \\ \mathrm{H} & -1.8571717 & -4.0959046 & 0.2709818 \\ \mathrm{H} & 1.1955670 & -1.6819601 & -2.6769195 \\ \mathrm{~N} & -1.3494918 & -1.9022299 & -1.2595871 \\ \mathrm{C} & 1.3857672 & -0.6281871 & -2.9210823 \\ \mathrm{~N} & -3.5681226 & -0.1875326 & -0.5372387 \\ \mathrm{C} & -1.5431279 & 1.8025588 & -2.5464605 \\ \mathrm{H} & -0.2690271 & 2.8942001 & -3.8998497 \\ \mathrm{C} & -0.1733380 & -1.7955213 & -0.6784947 \\ \mathrm{~N} & -0.5375324 & 0.7957406 & -2.1589438 \\ \mathrm{C} & -2.0024864 & 4.1164381 & -3.4465764 \\ \mathrm{O} & 0.6702182 & -2.6967941 & -0.4181398 \\ \mathrm{H} & -4.4853767 & -1.9052044 & 1.3348932 \\ \mathrm{C} & -0.9247271 & 3.1094912 & -3.0422574 \\ \mathrm{H} & -2.1695526 & 1.9845756 & -1.6661244 \\ \mathrm{H} & -1.5559358 & 5.0554603 & -3.8021663 \\ \mathrm{H} & 1.9641072 & -0.1963612 & -2.0977192 \\ \mathrm{H} & -2.6588062 & 4.3573652 & -2.5963285 \\ \mathrm{C} & -3.0507706 & -0.3939288 & 0.7973911 \\ \mathrm{H} & -2.1990573 & -1.0862696 & 0.7460537 \\ \mathrm{C} & -4.1207371 & -0.9471689 & 1.7399706 \\ \mathrm{H} & -4.9836033 & -0.2621093 & 1.7573531 \\ \mathrm{C} & -0.1964993 & 0.7534512 & -0.7844191 \\ \mathrm{~N} & 0.2071936 & -0.4388929 & -0.2490328 \\ \mathrm{H} & -0.2924196 & 3.5246441 & -2.2435921 \\ \mathrm{H} & -2.6812778 & 0.5698710 & 1.1845602 \\ \mathrm{H} & -0.2437972 & 1.8003484 & -0.1212673 \\ \mathrm{H} & 1.7823287 & -1.1489929 & 0.8867096 \\ & -0.3197754 & -1.5491025 & 2.1906666\end{array}$




$\begin{array}{lrrr}\mathrm{H} & -2.7349259 & -1.8586048 & 3.1566039 \\ \mathrm{H} & -4.3497905 & -1.5419267 & 3.8281477 \\ \mathrm{C} & 1.0139151 & -0.3703124 & 0.9791476 \\ \mathrm{C} & 0.2095062 & -0.5858196 & 2.2602219 \\ \mathrm{H} & -3.2100899 & -0.2010617 & 3.5776846 \\ \mathrm{H} & 1.5016823 & 0.6118900 & 1.0001869 \\ \mathrm{H} & -0.5593408 & 0.1964565 & 2.3371798 \\ \mathrm{H} & 1.8685423 & -1.3638378 & 3.4449301 \\ \mathrm{C} & 1.1062413 & -0.5715845 & 3.4980121 \\ \mathrm{H} & 0.5211919 & -0.7291739 & 4.4148655 \\ \mathrm{H} & 1.6312041 & 0.3909651 & 3.5963027\end{array}$

$$
\mathrm{TS}(10 \longrightarrow \text { 11) }
$$

56

Energy $=-1187.216366083$

$\begin{array}{lrrr}\mathrm{H} & 1.8015992 & -2.4810790 & -1.3925319 \\ \mathrm{H} & 1.7472827 & -0.0168981 & -0.9399982 \\ \mathrm{H} & -3.5934538 & 3.0566084 & 0.7308271 \\ \mathrm{H} & 3.5701711 & 0.7694431 & 5.2502837 \\ \mathrm{H} & -5.2937807 & 3.5552301 & -1.1742166 \\ \mathrm{H} & -3.0237791 & 4.7463710 & 0.6502479 \\ \mathrm{O} & 0.4094327 & 2.3200038 & 2.8268926 \\ \mathrm{H} & 0.2337806 & -0.5155481 & -1.6949061 \\ \mathrm{C} & 0.8375348 & -0.6159144 & -0.7777962 \\ \mathrm{C} & 1.2194524 & -2.0791422 & -0.5508770 \\ \mathrm{C} & -2.8866996 & 3.7381610 & 0.2401412 \\ \mathrm{H} & 2.0861618 & 0.7724525 & 3.1069229 \\ \mathrm{O} & -2.3519710 & 1.1811333 & 1.1883157 \\ \mathrm{H} & -4.7174776 & 5.2337789 & -1.2594550 \\ \mathrm{H} & 1.8282457 & -2.1891013 & 0.3600993 \\ \mathrm{C} & 4.3283182 & 1.3276708 & 4.6803752 \\ \mathrm{H} & 4.8827448 & 1.9598330 & 5.3875897\end{array}$




\begin{tabular}{|c|c|c|c|}
\hline & 1.6404387 & 4.9129394 & 3.4856111 \\
\hline & -4.5347077 & 4.2120126 & -1.6262143 \\
\hline & 0.4800140 & 6.0439577 & 2.7754720 \\
\hline & 2.9983281 & 2.9108063 & 4.0250329 \\
\hline & -1.3809768 & 1.8115597 & 0.6876871 \\
\hline & -1.5441860 & 3.2691144 & 0.6008049 \\
\hline & 0.3251833 & -2.7099417 & -0.4296785 \\
\hline & 1.0200698 & 2.6665699 & 1.8170908 \\
\hline & 5.0337474 & 0.5972725 & 4.2563515 \\
\hline & 2.4107745 & 7.2707762 & 3.8554581 \\
\hline ( & 3.6802079 & 2.1688474 & 3.5804472 \\
\hline & 1.5084070 & 5.6628922 & 2.6894428 \\
\hline$C$ & -3.1220670 & 3.7456904 & -1.2719764 \\
\hline$C$ & 2.8971474 & 1.3018793 & 2.5943322 \\
\hline C & -0.5629159 & 4.2231136 & 0.6940198 \\
\hline & 0.0522627 & -0.0287058 & 0.3968655 \\
\hline & -0.2405156 & 1.3799249 & 0.2006048 \\
\hline F & -4.6958214 & 4.2123943 & -2.7134256 \\
\hline 0 & -0.7926494 & 5.4161558 & 0.4117518 \\
\hline$\Pi$ & 0.6 & -0.100 & 1.3206535 \\
\hline $\mathrm{H}$ & -2.9489407 & 2.7303045 & -1.6642480 \\
\hline 1 & 0.7244888 & 3.8683750 & 1.1152719 \\
\hline ( & 2.5160293 & 6.7990987 & 2.8687890 \\
\hline $\mathrm{H}$ & 3.5580475 & 0.5710283 & 2.1166720 \\
\hline $\mathrm{N}$ & 2.2831744 & 2.1006144 & 1.5167225 \\
\hline $\mathrm{H}$ & -2.3738570 & 4.4019003 & -1.7431333 \\
\hline$C$ & 1.6711988 & 4.9822251 & 1.3293453 \\
\hline$H$ & -0.8713758 & -0.6176297 & 0.5509623 \\
\hline $\mathrm{H}$ & 4.4494600 & 2.7299434 & 3.0276352 \\
\hline $\mathrm{H}$ & 3.5493189 & 6.4299681 & 2.7809298 \\
\hline $\mathrm{H}$ & 2.3735699 & 7.5786163 & 2.1052179 \\
\hline $\mathrm{H}$ & 2.6906596 & 4.5853800 & 1.2403629 \\
\hline $\mathrm{H}$ & 1.5168762 & 5.7027786 & 0.51788 \\
\hline
\end{tabular}




$\begin{array}{lrrr}\text { C } & 2.9926332 & 2.1923770 & 0.3116356 \\ \text { H } & 1.2756735 & 2.4478838 & -0.9445153 \\ \text { C } & 2.3310584 & 2.7576682 & -0.9150038 \\ \text { O } & 4.1446215 & 1.7437951 & 0.2643287 \\ \text { H } & 2.8918928 & 2.3860592 & -1.7804598 \\ \text { H } & 2.3796072 & 3.8541953 & -0.9212088 \\ & & & \\ \text { TS(11 } \longrightarrow \mathbf{6 + 1 2}) & & \end{array}$

\section{6}

Energy $=-1187.235158055$

$\begin{array}{lrrr}\mathrm{H} & 1.2921569 & 1.6036158 & 3.1580279 \\ \mathrm{H} & 6.0974374 & 0.1749202 & 0.9747580 \\ \mathrm{H} & 0.9672156 & 0.5657379 & 1.7433409 \\ \mathrm{C} & 0.8536508 & 1.5757864 & 2.1542550 \\ \mathrm{O} & -1.0305216 & 2.4504765 & 3.3498495 \\ \mathrm{H} & 3.6092911 & -0.2073501 & 0.9068303 \\ \mathrm{H} & 1.4178743 & 2.2638841 & 1.5102278 \\ \mathrm{H} & 3.9867577 & 1.4931058 & 0.5930671 \\ \mathrm{C} & -0.6066568 & 1.9928792 & 2.2431622 \\ \mathrm{C} & 5.5629080 & 0.1162924 & 0.0166174 \\ \mathrm{C} & 4.0864464 & 0.4749284 & 0.1865337 \\ \mathrm{H} & 5.6803258 & -0.9065283 & -0.3723646 \\ \mathrm{H} & -0.3365940 & 4.2816706 & 1.0016648 \\ \mathrm{H} & -2.2372017 & -0.5738905 & -0.4008860 \\ \mathrm{H} & 1.2370126 & 4.6370051 & 0.2686905 \\ \mathrm{H} & 6.0585529 & 0.8013826 & -0.6878326 \\ \mathrm{H} & -0.0486700 & 6.7617007 & 0.6894354 \\ \mathrm{O} & 1.4083630 & -1.4625901 & -0.5850720 \\ \mathrm{H} & -3.3132844 & 1.0356188 & 2.8763551 \\ \mathrm{C} & 0.1511626 & 4.6717257 & 0.0977096 \\ \mathrm{H} & -1.0771400 & -1.8275847 & -0.8889150 \\ \mathrm{~N} & -1.3601460 & 1.8654846 & 1.1491421\end{array}$




$\begin{array}{lrrr}\mathrm{C} & 1.0179835 & -0.2890729 & -0.6790458 \\ \mathrm{C} & -1.2483888 & -0.9973364 & -0.1884617 \\ \mathrm{H} & -2.8059870 & 3.2484718 & 1.7875978 \\ \mathrm{C} & 3.3316564 & 0.3975104 & -1.1410436 \\ \mathrm{~N} & -0.2852148 & 0.0680332 & -0.4917069 \\ \mathrm{C} & -0.2943399 & 6.1115793 & -0.1617881 \\ \mathrm{~N} & 1.9096261 & 0.7378742 & -0.9999900 \\ \mathrm{H} & -3.2339997 & -2.2213335 & 1.3299479 \\ \mathrm{H} & 3.3802693 & -0.6170364 & -1.5526360 \\ \mathrm{C} & 1.5427235 & 2.0814942 & -1.1114719 \\ \mathrm{~N} & 0.2235489 & 2.3722410 & -0.9220010 \\ \mathrm{C} & -2.7392642 & 2.2997935 & 1.2248561 \\ \mathrm{H} & -1.3817787 & 6.1672850 & -0.3238787 \\ \mathrm{C} & -3.6801169 & 1.2703819 & 1.8653417 \\ \mathrm{C} & -0.8280342 & 1.3978689 & -0.6937905 \\ \mathrm{O} & 2.3849855 & 2.9496631 & -1.3859408 \\ \mathrm{H} & 3.7597128 & 1.0968870 & -1.8683037 \\ \mathrm{H} & -5.2016109 & 2.6865392 & 2.5287867 \\ \mathrm{H} & 0.1978533 & 6.5266057 & -1.0546970 \\ \mathrm{H} & -2.0573832 & -3.4577072 & 0.8354298 \\ \mathrm{H} & -0.2654673 & 4.1603158 & -2.0039535 \\ \mathrm{C} & -0.1985200 & 3.7689179 & -1.0870733 \\ \mathrm{C} & -2.2136794 & -2.5969584 & 1.5036388 \\ \mathrm{H} & -3.6294585 & 0.3365185 & 1.2832724 \\ \mathrm{C} & -1.1753534 & -1.5032430 & 1.2528138 \\ \mathrm{C} & -5.1247701 & 1.7674824 & 1.9271178 \\ \mathrm{H} & -1.2832335 & 3.7374715 & -1.2431860 \\ \mathrm{H} & -5.7927832 & 1.0176218 & 2.3752291 \\ \mathrm{H} & -1.3459333 & -0.6551794 & 1.9298240 \\ \mathrm{H} & -3.0707397 & 2.4874660 & 0.1916537 \\ \mathrm{H} & -1.832772 & -2.9619154 & 2.5389266 \\ \mathrm{H} & -1.8869628 & 1.4496666\end{array}$




$$
\begin{aligned}
& \begin{array}{llll}
\mathrm{H} & -5.5073050 & 1.9978513 & 0.9203695
\end{array} \\
& \mathrm{TS}(9 \longrightarrow 24+12)
\end{aligned}
$$

43

Energy $=-900 \cdot 3964587378$

$\mathrm{H} \quad 2.5826439 \quad-2.2211886 \quad-1.6086001$

$\mathrm{H} \quad \begin{array}{llll}1.2785342 & -3.4278887 & -1.5714525\end{array}$

$\begin{array}{llll}\text { C } & 1.8346398 & -2.7030225 & -0.9603746\end{array}$

$\begin{array}{llll}0 & 1.1154604 & 1.5432068 & -0.8750798\end{array}$

$\mathrm{H} \quad 0.3523704 \quad-1.1240027 \quad-1.1250850$

$\begin{array}{llll}\text { C } & 0.8984524 & -1.6666140 & -0.3385717\end{array}$

$\mathrm{H} \quad-1.5149340 \quad 1.2410854 \quad-2.1823210$

$\mathrm{H} \quad 2.4071790 \quad-0.1309011 \quad-0.1031448$

$\mathrm{H} \quad 2.3779286 \quad-3.2643410 \quad-0.1847365$

$\begin{array}{llll}\mathrm{H} & 0.7639441 & 4.6535196 & 0.4282084\end{array}$

$\begin{array}{llll}\text { C } & 0.4862711 & 1.5005911 & 0.2018995\end{array}$

$\begin{array}{llll}\text { C } & 1.6622506 & -0.6482232 & 0.5106151\end{array}$

$\mathrm{H} \quad-3.0103825 \quad-0.4426291 \quad-3.3616455$

C $\quad-2.2333395 \quad 1.3397147 \quad-1.3358893$

$\begin{array}{llll}\mathrm{N} & 0.0464142 & 2.7318648 & 0.8551052\end{array}$

$\mathrm{H} \quad-2.9027381 \quad 2.1825128 \quad-1.5979492$

$\begin{array}{llll}\mathrm{H} & 0.1446621 & -2.1643698 & 0.2914041\end{array}$

C $\quad 0.9584612 \quad 3.8292065 \quad 1.1382193$

$\begin{array}{llll}\text { C } & -1.2039001 & 2.8866714 & 0.2391889\end{array}$

$\begin{array}{llll}0 & -1.8093485 & 3.9720719 & 0.0938035\end{array}$

$\begin{array}{llll}\mathrm{N} & -1.5068616 & 1.6196404 & -0.1259838\end{array}$

$\begin{array}{llll}C & -3.7404424 & -0.3219462 & -2.5460850\end{array}$

$\begin{array}{llll}\mathrm{N} & 0.7733983 & 0.3792585 & 1.0803323\end{array}$

$\mathrm{H} \quad-4.4511681 \quad 0.4618189 \quad-2.8512002$

$\mathrm{H} \quad 1.9823080 \quad 3.4729757 \quad 0.9470431$

$\mathrm{H} \quad 2.1640091 \quad-1.1482596 \quad 1.3466181$

$\begin{array}{llll}\mathrm{H} & 1.5416662 & 6.3810619 & 2.1271911\end{array}$ 


$\begin{array}{lrrr}\mathrm{C} & -3.0431082 & 0.0450777 & -1.2356607 \\ \mathrm{H} & -0.2032285 & 4.6524079 & 2.7521306 \\ \mathrm{C} & 0.8432809 & 4.3619671 & 2.5686397 \\ \mathrm{H} & -2.3611360 & -0.7644894 & -0.9284025 \\ \mathrm{H} & -4.3001072 & -1.2640199 & -2.4565036 \\ \mathrm{C} & 1.7716990 & 5.5521783 & 2.8142150 \\ \mathrm{C} & 0.1842650 & 0.0757589 & 2.2947787 \\ \mathrm{H} & -1.6547722 & 1.1669627 & 2.0789436 \\ \mathrm{H} & 2.8247067 & 5.2720190 & 2.6571574 \\ \mathrm{H} & -3.7826468 & 0.1531403 & -0.4252955 \\ \mathrm{O} & 0.5414126 & -0.9438815 & 2.9158341 \\ \mathrm{C} & -0.9164354 & 0.9364330 & 2.8585569 \\ \mathrm{H} & 1.0762300 & 3.5495033 & 3.2753863 \\ \mathrm{H} & 1.6748096 & 5.9309674 & 3.8413061 \\ \mathrm{H} & -1.3747992 & 0.3777350 & 3.6823671 \\ \mathrm{H} & -0.5221405 & 1.8863142 & 3.2377942\end{array}$

\subsubsection{Catalyst Migration}

\section{Compound 13}

\begin{tabular}{lrrr}
43 & \\
\multicolumn{4}{l}{ Energy $=-900.4376389642$} \\
H & 3.7739568 & -1.0996145 & 1.6205048 \\
H & 4.0915720 & -0.4573723 & -0.0056101 \\
$\mathrm{C}$ & 3.6920484 & -0.2021580 & 0.9880427 \\
$\mathrm{H}$ & 4.3404823 & 0.5725864 & 1.4207700 \\
$\mathrm{H}$ & 1.3586553 & -1.7028545 & 0.9150152 \\
$\mathrm{H}$ & 1.6781071 & -1.0600820 & -0.7164833 \\
$\mathrm{C}$ & 1.3336572 & -0.7990539 & 0.2924912 \\
$\mathrm{C}$ & 2.2424776 & 0.2756547 & 0.8936573 \\
$\mathrm{H}$ & 1.8617008 & 0.5379528 & 1.8919226 \\
$\mathrm{H}$ & 2.1783820 & 1.1783084 & 0.2679362 \\
$\mathrm{O}$ & 0.4375398 & 0.7833785 & -1.7379855
\end{tabular}




\begin{tabular}{|c|c|c|c|}
\hline & -0.0711535 & -0.3973271 & 68 \\
\hline & -0.3762307 & -0.8475831 & 2.4149775 \\
\hline & -0.4386699 & 0.3770724 & 57593 \\
\hline & -0.8833485 & -0.5142855 & 1.3230469 \\
\hline & -1.4973437 & 2.3616625 & -2.1976245 \\
\hline & -1.7565947 & 0.6417656 & -1.0592136 \\
\hline & -2.1879358 & -0.2233140 & 10777 \\
\hline $\mathrm{F}$ & -2.5405469 & 0.2701363 & 3.1291988 \\
\hline ( & -2.2159949 & 1.5330894 & -2.1117011 \\
\hline $\mathrm{E}$ & -1.4473622 & 0.3796791 & -3.7643352 \\
\hline & -2.8257696 & -0.16383 & -0.2 \\
\hline & -3.0781886 & -0.1956820 & 2.2899621 \\
\hline 0 & -3.9822081 & 0.4186587 & -0.2704389 \\
\hline $\mathrm{H}$ & -2.7511693 & -2.2374464 & 2.9024086 \\
\hline $\mathrm{H}$ & -2.0529727 & 2.6644511 & -4.6680715 \\
\hline C & -2.3935281 & 0.8658814 & -3.4807770 \\
\hline & -3.1790906 & 1.9325098 & -1.7629707 \\
\hline$C$ & -3.6068501 & -1.5690789 & 2.7207947 \\
\hline C & -2.8120905 & 1.8757338 & -4.5501580 \\
\hline $\mathrm{H}$ & -3.90739 & -1.0594 & 88 \\
\hline $\mathrm{H}$ & -3.9165487 & 0.4538641 & 2.0005118 \\
\hline$C$ & -4.4781062 & -1.4703761 & 3.9736425 \\
\hline$C$ & -2.8267126 & -1.6065510 & -0.8398028 \\
\hline H & -3.1517399 & 0.0721696 & -3.3972032 \\
\hline & -1.8309120 & -2.0728725 & -0.8293839 \\
\hline $\mathrm{H}$ & -2.9482132 & 1.3924021 & -5.5279525 \\
\hline$H$ & -4.1875374 & -2.0057734 & 1.8936847 \\
\hline$H$ & -3.7613979 & 2.3652856 & -4.2829019 \\
\hline I & -4.8625703 & -2.4552260 & 4.2745037 \\
\hline $\mathrm{H}$ & -3.5114187 & -2.2204626 & -0.2394585 \\
\hline $\mathrm{H}$ & -5.3428262 & -0.8103182 & 3.8039798 \\
\hline & -3.1937048 & -1.5786931 & -1.8745830 \\
\hline
\end{tabular}




\section{Compound 14}

\begin{tabular}{|c|c|c|c|}
\hline \multicolumn{4}{|c|}{ Energy $=-1187.225068140$} \\
\hline & -4.6797853 & -2.3533750 & -3.0841152 \\
\hline F & -3.3875341 & -0.8074720 & -6.1319058 \\
\hline & -5.2425059 & -2.9387993 & -1.5103277 \\
\hline & -4.4036975 & -2.9607717 & -2.2148679 \\
\hline & -6.2396699 & -3.9308594 & 1.8305090 \\
\hline C & -2.4969961 & -1.4480748 & -6.2234323 \\
\hline $\mathrm{H}$ & -4.2244384 & -3.9935827 & -2.5355709 \\
\hline & -3.1046068 & -0.9420916 & -3.5505058 \\
\hline & -2.5934946 & -2.0264506 & -7.1526801 \\
\hline $\mathrm{H}$ & -4.5326102 & -2.5120570 & 0.4834454 \\
\hline c & -5.4381779 & -4.6671102 & 1.9956407 \\
\hline 0 & -3.4996928 & -1.0701757 & -1.1708781 \\
\hline & -3.2236371 & -3.0373231 & -4.9367647 \\
\hline & -1.6212871 & -0.7892932 & -6.3264288 \\
\hline$c$ & -2.3523481 & -2.3682382 & -5.0104970 \\
\hline & -5.9068505 & -5.6549272 & 2.1047605 \\
\hline $\mathrm{H}$ & -4.9336964 & -4.9298833 & -0.0982243 \\
\hline C & -2.2180671 & -1.5662408 & -3.7158011 \\
\hline & -3.1364199 & -2.4035788 & -1.5549852 \\
\hline & -4.4330677 & -4.6554174 & 0.8429931 \\
\hline & -3.7787009 & -3.2822405 & 0.6867794 \\
\hline & -2.9946081 & 1.0117882 & -0.5314560 \\
\hline $\mathrm{H}$ & -4.9488351 & -4.4195343 & 2.9499389 \\
\hline C & -2.5251648 & -0.1577049 & -0.6138022 \\
\hline & -1.3533756 & -0.8909169 & -3.7808065 \\
\hline & -2.0409027 & -2.4046237 & -2.5216591 \\
\hline & -2.7882227 & -3.2249191 & -0.3971218 \\
\hline & -3.2720045 & -3.0017328 & 1.6208355 \\
\hline & -1.4649143 & -3.0086400 & -5.1222119 \\
\hline
\end{tabular}




$\begin{array}{lrrr}\mathrm{N} & -1.3806941 & -0.6869582 & -0.2919029 \\ \mathrm{H} & -3.6453499 & -5.4034092 & 1.0170011 \\ \mathrm{H} & 0.8275779 & -0.0758484 & -1.6100215 \\ \mathrm{H} & -0.1952470 & 1.3588695 & -1.6914245 \\ \mathrm{C} & -0.7591658 & -2.7918727 & -2.2462087 \\ \mathrm{C} & 0.4703260 & 0.7867423 & -1.0243185 \\ \mathrm{C} & -1.5224828 & -3.6275901 & -0.0802362 \\ \mathrm{C} & -0.3632058 & 0.2515892 & 0.1485575 \\ \mathrm{O} & 0.1771982 & -2.5786134 & -3.0299241 \\ \mathrm{~N} & -0.5578414 & -3.5171656 & -1.0760938 \\ \mathrm{H} & 0.3171159 & -0.2732983 & 0.8431741 \\ \mathrm{O} & -1.2467118 & -4.1381554 & 1.0161868 \\ \mathrm{H} & 2.2333773 & 2.0196876 & -1.4324578 \\ \mathrm{C} & 1.6481442 & 1.6529530 & -0.5763700 \\ \mathrm{H} & -0.7920756 & 1.1132334 & 0.7003437 \\ \mathrm{H} & 2.3300060 & 1.0828238 & 0.0742270 \\ \mathrm{C} & 0.8076986 & -3.9512118 & -0.7613052 \\ \mathrm{H} & 1.3045762 & 2.5303339 & -0.0062488 \\ \mathrm{H} & \mathbb{0} .7227763 & -4.8505778 & -0.1395116 \\ \mathrm{H} & 1.2833924 & -4.2226223 & -1.7113031 \\ \mathrm{H} & 1.1079056 & -2.6007580 & 0.8853745 \\ \mathrm{C} & 1.6294196 & -2.8817771 & -0.0412162 \\ \mathrm{H} & 1.6650587 & -1.9809327 & -0.6707581 \\ \mathrm{C} & 3.0430023 & -3.3736595 & 0.2685679 \\ \mathrm{H} & 3.0216098 & -4.2667563 & 0.9119223 \\ \mathrm{H} & 3.5823441 & -3.6397939 & -0.6534979 \\ \mathrm{H} & 3.6276752 & -2.6011779 & 0.7873546\end{array}$

\section{Compound 15}

57

Energy $=-1187.720480287$

$\mathrm{H} \quad 3.0865686 \quad-3.4437529 \quad-5.3618290$ 


\begin{tabular}{|c|c|c|c|}
\hline & 3.2436032 & -5.0921466 & -4.7 \\
\hline C & 197 & -4.297 & 76388 \\
\hline H & 5.9440353 & -1.3141737 & 1.6497799 \\
\hline & 1.8633099 & -4.6723395 & -5.7529354 \\
\hline & .2017048 & -2.5011232 & -2.9843697 \\
\hline & 3.3531568 & -4.1257329 & -2.3080417 \\
\hline H & 7.1744592 & -2.5902892 & 1.7218729 \\
\hline 0 & 3.6467961 & -1.6533039 & 0.5810323 \\
\hline & .6890592 & 0. 3354244 & 0.6763830 \\
\hline C & 6.4149420 & -2.1255804 & 1.0771467 \\
\hline C & 2.6286207 & -3.3624114 & -2.6166064 \\
\hline C & 1.7157916 & -3.8891190 & -3.7239377 \\
\hline C & 4.1141479 & -2.7881577 & 0.5253568 \\
\hline $\mathrm{N}$ & 5.3922990 & -3.1289641 & 0.8075787 \\
\hline $\mathrm{H}$ & 8.9289239 & -0.5 & 1206 \\
\hline $\mathrm{H}$ & 0.9907599 & -3.1042127 & -3.9844769 \\
\hline C & 8.1253092 & -0.5125737 & 0.1271239 \\
\hline $\mathrm{H}$ & 5.6744742 & -4.0890841 & 0.6351576 \\
\hline 0 & 3.3979662 & -3.9156536 & $8.10 J 4754$ \\
\hline 0 & 1.5075034 & -0.9191241 & -2.4297090 \\
\hline $\mathrm{H}$ & 1.1383665 & -4.7472637 & -3.3486088 \\
\hline $\mathrm{H}$ & -1.8363220 & -0.4745283 & -0.0709984 \\
\hline C & 7.0632410 & -1.5651576 & -0.1 \\
\hline $\mathrm{N}$ & 1.901 & 31000 & -1.4 \\
\hline $\mathrm{H}$ & 6.2748228 & -1.1299474 & -0.8268171 \\
\hline $\mathrm{H}$ & 8.5827356 & -0.1188580 & -0.7909912 \\
\hline $\mathrm{H}$ & 2.2121383 & -5.6122021 & -1.3441636 \\
\hline C & 1.4167431 & -1.6384262 & -1.4292590 \\
\hline $\mathrm{H}$ & -2.8696072 & 1.5969237 & -1.0611276 \\
\hline C & 1.9776557 & -3.7594889 & -0.2385258 \\
\hline $\mathrm{H}$ & 7.5098776 & -2.3955670 & -0.7625996 \\
\hline C & -1.2473741 & 0.1764856 & -0.735527 \\
\hline & -1.7843390 & 2.0331719 & 0.27690 \\
\hline
\end{tabular}




$\begin{array}{lrrr}\mathrm{H} & -1.3068838 & -0.2549537 & -1.7467238 \\ \mathrm{C} & 1.5741820 & -5.1986771 & -0.5571477 \\ \mathrm{C} & -1.8216243 & 1.5936679 & -0.7313420 \\ \mathrm{~N} & 0.7845175 & -1.1920701 & -0.2750395 \\ \mathrm{H} & 0.2983661 & 0.5481956 & 0.7446948 \\ \mathrm{H} & 1.6820986 & -5.8337942 & 0.3274461 \\ \mathrm{C} & 0.2108135 & 0.1611812 & -0.2769261 \\ \mathrm{~N} & 1.1869006 & -3.2231325 & 0.8425337 \\ \mathrm{H} & 1.9595384 & -4.7139751 & 2.0829971 \\ \mathrm{H} & 0.5302161 & -5.2095594 & -0.8890347 \\ \mathrm{C} & 0.6881040 & -1.9449608 & 0.8891863 \\ \mathrm{H} & -1.2542059 & 2.2529622 & -1.4054933 \\ \mathrm{C} & 1.1372369 & -3.9885827 & 2.1015429 \\ \mathrm{H} & 1.3435284 & -3.2863023 & 2.9199710 \\ \mathrm{H} & 0.8311757 & 0.7715934 & -0.9430680 \\ \mathrm{O} & 0.1489535 & -1.4900170 & 1.9036573 \\ \mathrm{H} & -0.4075395 & -5.3820931 & 1.5139628 \\ \mathrm{C} & -0.2027556 & -4.6844080 & 2.3398384 \\ \mathrm{H} & \mathbb{0} .5770423 & -6.2028766 & 3.6999124 \\ \mathrm{H} & -0.9996982 & -3.9265427 & 2.3241672 \\ \mathrm{C} & -0.2083780 & -5.4323772 & 3.6736015 \\ \mathrm{H} & -0.0310263 & -4.7443851 & 4.5139870 \\ \mathrm{H} & -1.1731038 & -5.9295901 & 3.8434419\end{array}$

\section{Compound 16}

L1
$\begin{array}{lrrr}\text { Energy }=-824.4254479306 \\ \text { H } & -5.2757744 & -3.0373232 & 4.0395128 \\ \text { H } & -4.5691197 & -0.7340509 & 3.3171721 \\ \text { H } & -3.7962286 & 3.7455359 & 2.7652118 \\ \text { C } & -5.2558479 & -2.7637516 & 2.9758637 \\ \text { C } & -4.2796580 & -1.6143149 & 2.7238852\end{array}$




\begin{tabular}{|c|c|c|c|}
\hline & -3.2659892 & -1.8977051 & 6253 \\
\hline & -3.6781920 & 6.2546588 & 2.8955717 \\
\hline & 2.0999884 & -0.3025450 & -2.7443290 \\
\hline & -6.2789391 & -2.4869632 & 2.6798464 \\
\hline & 0.1373333 & 1.0509904 & -1.9361425 \\
\hline & -5.7528253 & 0.6819797 & 1.8462318 \\
\hline & -3.2609380 & 4.3164716 & 1.9918766 \\
\hline & -2.1973288 & 4.3194715 & 2.2751019 \\
\hline & -0.1654099 & -0.6807421 & -1.7156828 \\
\hline & -3.7956604 & 474451 & 1.9283685 \\
\hline & -5.0829949 & 1.4737257 & 1.5338393 \\
\hline & -5.4441294 & 2.4911274 & 1.6211896 \\
\hline C & 0.4632802 & 0. 1583769 & -1.3806213 \\
\hline & -4.9713654 & -3.6587360 & 2.4022930 \\
\hline & 1.9354130 & -0.1361912 & -1.6709815 \\
\hline & -4.8649687 & 5.7598683 & 1.6688658 \\
\hline C & -3.8367287 & 1.2047814 & 1.0805744 \\
\hline ( & -4.2316989 & -1.2430804 & 1.240 \\
\hline $\mathrm{N}$ & -3.3 & -0.1 & 109 \\
\hline & -2.9461810 & 2.2126154 & 0.6696870 \\
\hline C & -2.0291517 & -0.4111059 & 0.6661854 \\
\hline F & 2.5766481 & 0.7013692 & -1.3575060 \\
\hline $\mathrm{H}$ & -3.2596305 & 6.3379856 & 1.1701992 \\
\hline C & -3.4012225 & 3.6112789 & 0.6413719 \\
\hline 0 & -1.6127453 & -1.5709726 & 0.6227389 \\
\hline F & -5.2280944 & -0.9888711 & 0.8566682 \\
\hline 1 & 2.2725567 & -1.0360407 & -1.1346897 \\
\hline C & -1.6184778 & 1.9927131 & 0.3667253 \\
\hline $\mathrm{N}$ & -1.1847748 & 0.6702956 & 0.4253929 \\
\hline C & 0.2257915 & 0.3893975 & 0.1116964 \\
\hline $\mathrm{H}$ & -4.4414921 & 3.6149453 & 0.2915078 \\
\hline 0 & -0.8480557 & 2.9067080 & 0.0642327 \\
\hline $\mathrm{H}$ & -3.8541280 & -2.0884142 & 0.6546181 \\
\hline
\end{tabular}




$\begin{array}{rrrr}\mathrm{H} & -2.7936165 & 4.1222629 & -0.1135664 \\ \mathrm{H} & 0.8076782 & 1.2469418 & 0.4684284 \\ \mathrm{H} & 0.5093875 & -0.4972082 & 0.6902738\end{array}$

\section{Compound $17^{*}$}

15

Energy $=-362.7868711315$

$\begin{array}{lrrr}\mathrm{H} & 1.1976634 & 1.8077519 & -0.5985338 \\ \mathrm{H} & -0.4403156 & 2.0011487 & 0.0265892 \\ \mathrm{C} & 0.1870680 & 1.3459911 & -0.6150519 \\ \mathrm{~N} & 0.2167910 & -0.0214707 & -0.1146427 \\ \mathrm{O} & 1.0522717 & 0.7111671 & 1.9578537 \\ \mathrm{C} & 0.6523612 & -0.1442161 & 1.1206210 \\ \mathrm{H} & 0.6009512 & 3.2657938 & -2.6390106 \\ \mathrm{H} & -1.0476738 & 3.4628689 & -2.0089348 \\ \mathrm{O} & 0.6736421 & -1.4813506 & 1.5902592 \\ \mathrm{C} & -0.4017529 & 2.8110965 & -2.6177245 \\ \mathrm{H} & 0.3414072 & -2.0171396 & 0.8425815 \\ \mathrm{C} & -0.3521280 & 1.3937359 & -2.0446018 \\ \mathrm{H} & 0.2784903 & 0.7506162 & -2.6816386 \\ \mathrm{H} & -0.7935339 & 2.8188338 & -3.6451558 \\ \mathrm{H} & -1.3602341 & 0.9458286 & -2.0567547\end{array}$

\section{Compound 17}

$\begin{array}{lrrr}15 & & \\ \text { Energy }=-362.8153716420 & \\ \text { H } & 1.0313741 & 0.6195965 & 1.3373695 \\ \text { H } & 2.1916820 & 2.5391461 & 0.1721815 \\ \text { H } & 2.0382536 & 0.0317184 & 0.0052913 \\ \text { C } & 1.1378946 & 0.6239012 & 0.2406619 \\ \text { C } & 1.3080650 & 2.0562300 & -0.2688534 \\ \text { O } & -0.5766695 & -2.9088489 & 1.8037316\end{array}$




$\begin{array}{lrrr}\mathrm{H} & 0.4496551 & -2.0991578 & -0.2010620 \\ \mathrm{C} & -0.8074234 & -1.7356898 & 1.3582130 \\ \mathrm{~N} & -0.2790219 & -1.4462658 & 0.0799861 \\ \mathrm{H} & 0.4299062 & 2.6716422 & -0.0197101 \\ \mathrm{C} & -0.0867836 & -0.0727643 & -0.3662266 \\ \mathrm{O} & -1.4958317 & -0.8354466 & 1.9311007 \\ \mathrm{H} & 1.4274865 & 2.0754353 & -1.3633057 \\ \mathrm{H} & -0.9953882 & 0.4872799 & -0.1024097 \\ \mathrm{H} & -0.0042979 & -0.0712605 & -1.4661821\end{array}$

\section{Compound $17+\mathrm{H}^{+}$}

16

\begin{tabular}{lrrr}
\multicolumn{4}{l}{ Energy $=-363.2837786078$} \\
$\mathrm{H}$ & -1.9552953 & -3.6104255 & 1.7530692 \\
$\mathrm{O}$ & -1.1117177 & -3.2830576 & 1.3822346 \\
$\mathrm{C}$ & -1.3296682 & -1.9828361 & 1.0067180 \\
$\mathrm{H}$ & 0.6014002 & -2.0261282 & 0.3792867 \\
$\mathrm{O}$ & -2.4193659 & -1.4220425 & 1.1483948 \\
$\mathrm{H}$ & -1.4703385 & -0.5109402 & -1.7588477 \\
$\mathrm{~N}$ & -0.2101661 & -1.4273249 & 0.4981061 \\
$\mathrm{H}$ & 0.2539572 & -0.7236905 & -2.0939320 \\
$\mathrm{C}$ & -0.4747229 & -0.0733967 & -1.5839318 \\
$\mathrm{C}$ & -0.1874942 & -0.0895403 & -0.0793441 \\
$\mathrm{H}$ & -0.6188170 & 1.3407952 & -3.2383670 \\
$\mathrm{C}$ & -0.4095978 & 1.3418784 & -2.1598066 \\
$\mathrm{H}$ & -0.9322386 & 0.5155070 & 0.4559477 \\
$\mathrm{H}$ & 0.8022317 & 0.3453642 & 0.1231820 \\
$\mathrm{H}$ & -1.1447811 & 2.0017201 & -1.6747583 \\
$\mathrm{H}$ & 0.5870356 & 1.7845149 & -2.0109206
\end{tabular}

$\mathrm{TS}(9 \longrightarrow$ 13)

43 


\begin{tabular}{lrrr}
\multicolumn{4}{l}{ Energy $=-900.4166606328$} \\
$\mathrm{C}$ & -2.9119803 & -0.9125294 & -1.3728081 \\
$\mathrm{~N}$ & -1.8750906 & -0.0161448 & -1.5332496 \\
$\mathrm{C}$ & -0.5228876 & -0.2741050 & -1.1767703 \\
$\mathrm{~N}$ & -0.2451249 & -0.6329329 & 0.1105044 \\
$\mathrm{C}$ & -1.1299475 & -0.5433797 & 1.3175732 \\
$\mathrm{~N}$ & -2.4129960 & -0.5605407 & 1.0824361 \\
$\mathrm{C}$ & 1.1595432 & -0.9812786 & 0.3775873 \\
$\mathrm{C}$ & 2.0602979 & 0.2286693 & 0.6343316 \\
$\mathrm{C}$ & 3.5088740 & -0.1918032 & 0.8864209 \\
$\mathrm{O}$ & 0.3654382 & -0.1050291 & -2.0307572 \\
$\mathrm{O}$ & -0.4873868 & -0.4663321 & 2.4067574 \\
$\mathrm{C}$ & -2.1779726 & 1.3534100 & -1.9665815 \\
$\mathrm{C}$ & -2.2674774 & 1.5117593 & -3.4850801 \\
$\mathrm{C}$ & -2.5983627 & 2.9503165 & -3.8847974 \\
$\mathrm{C}$ & -3.2623958 & -0.4756550 & 2.2553818 \\
$\mathrm{C}$ & -3.5009713 & -1.8397314 & 2.9203206 \\
$\mathrm{C}$ & -4.4612963 & -1.7613353 & 4.1083363 \\
$\mathrm{O}$ & -4.0806941 & -0.5664845 & -1.6041256 \\
$\mathrm{C}$ & -2.5660079 & -2.3715981 & -1.1907726 \\
$\mathrm{H}$ & 3.5831776 & -0.8544906 & 1.7625714 \\
$\mathrm{H}$ & 3.9209346 & -0.7355544 & 0.0221701 \\
$\mathrm{H}$ & 4.1522272 & 0.6801512 & 1.0706987 \\
$\mathrm{H}$ & 1.1634979 & -1.6379868 & 1.2549755 \\
$\mathrm{H}$ & 1.5291225 & -1.5416351 & -0.4925937 \\
$\mathrm{H}$ & 1.6617663 & 0.7740947 & 1.5022625 \\
$\mathrm{H}$ & 2.0060049 & 0.9008701 & -0.2349458 \\
$\mathrm{H}$ & -1.3944514 & 2.0109907 & -1.5633557 \\
$\mathrm{H}$ & -2.8471587 & 0.2129861 & 3.0199103 \\
$\mathrm{H}$ & -1.3094479 & 1.1998200 & -3.9272752 \\
$\mathrm{H}$ & -1.8189549 & 3.6470863 & -3.5399761 \\
$\mathrm{H}$ & -1309703 & 1.6340442 & -1.4958995
\end{tabular}




$\begin{array}{lrrr}\text { H } & -4.0577559 & -1.1142672 & 4.9027114 \\ \mathrm{H} & -4.2394524 & -0.0650394 & 1.9420856 \\ \mathrm{H} & -3.0386273 & 0.8239703 & -3.8649035 \\ \mathrm{H} & -1.5559029 & -2.5438779 & -\mathbf{0 . 8 0 8 4 5 0 3} \\ \mathrm{H} & -2.6800375 & 3.0520817 & -4.9759991 \\ \mathrm{H} & -3.8962420 & -2.5324178 & 2.1590006 \\ \mathrm{H} & -3.5539257 & 3.2748220 & -3.4449603 \\ \mathrm{H} & -4.6472170 & -2.7524727 & 4.5474896 \\ \mathrm{H} & -3.2924114 & -2.8183807 & -\mathbf{0 . 5 0 4 9 6 1 8} \\ \mathrm{H} & -5.4336028 & -1.3433414 & 3.8032419 \\ \mathrm{H} & -2.6629358 & -2.8621583 & -2.1731946 \\ & & & \\ \mathrm{TS}\left(\mathbf{2}+\mathrm{H}_{2} \mathbf{O}+\mathbf{1 2} \longrightarrow \mathbf{1 7}^{\star}+\mathbf{1 2 + H ^ { + }}\right)\end{array}$

33

Energy $=-690.0848673886$

$\begin{array}{llll}\mathrm{O} & -2.8994113 & -1.4842698 & -3.7389032 \\ \mathrm{H} & -9.0203497 & -2.2159983 & -1.4417210 \\ \mathrm{H} & -8.3869694 & -2.7269164 & 0.1378008 \\ \mathrm{H} & -6.4851504 & -2.1051242 & -1.3923778 \\ \mathrm{C} & -8.4731286 & -1.8734728 & -0.5497159 \\ \mathrm{C} & -2.2256206 & -0.8243700 & -3.0146238 \\ \mathrm{C} & -7.0988922 & -1.3181772 & -0.9258748 \\ \mathrm{O} & -3.8173239 & -0.6740450 & -1.2334894 \\ \mathrm{H} & -7.7412804 & -0.4428677 & -2.7999022 \\ \mathrm{H} & -4.9044285 & -0.1031435 & -1.7601183 \\ \mathrm{H} & -3.2622196 & 0.0402767 & -0.8778896 \\ \mathrm{C} & -7.2024406 & -0.1329424 & -1.8841642 \\ \mathrm{H} & -9.0898813 & -1.1063802 & -0.0563713 \\ \mathrm{H} & -6.5529991 & -1.0040672 & -0.0221449 \\ \mathrm{~N} & -1.3790231 & -0.1524701 & -2.4359303 \\ \mathrm{H} & 0.6459182 & -0.5878063 & -2.7945273 \\ \mathrm{~N} & -5.8889172 & 0.3945240 & -2.2261008\end{array}$




$\begin{array}{lrll}\mathrm{C} & -0.0701639 & 0.2257017 & -2.9983582 \\ \mathrm{H} & -0.1380184 & 0.3392161 & -4.0930296 \\ \mathrm{H} & -4.2383174 & 1.9398694 & -4.3969289 \\ \mathrm{C} & -5.8173688 & 1.4673156 & -3.0149863 \\ \mathrm{H} & -7.8251697 & 0.6605937 & -1.4295422 \\ \mathrm{C} & -4.4219707 & 1.9903584 & -3.3136138 \\ \mathrm{H} & -3.6427740 & 1.4226474 & -2.7921068 \\ \mathrm{O} & -6.8202150 & 2.0565675 & -3.5142328 \\ \mathrm{C} & 0.4302450 & 1.5196777 & -2.3650908 \\ \mathrm{H} & 2.5535475 & 1.1317914 & -2.6914975 \\ \mathrm{H} & 0.4645860 & 1.3868119 & -1.2721160 \\ \mathrm{H} & -4.3619707 & 3.0489222 & -3.0213334 \\ \mathrm{C} & 1.8091538 & 1.9168154 & -2.8942828 \\ \mathrm{H} & 1.7858641 & 2.0830279 & -3.9819347 \\ \mathrm{H} & -0.2997371 & 2.3199169 & -2.5646030 \\ \mathrm{H} & 2.1614931 & 2.8435694 & -2.4208130\end{array}$

\section{$\operatorname{TS}\left(17^{\star} \longrightarrow 17\right)$}

$\begin{array}{lrrr}15 & & \\ \text { Energy }=-362.6682316469 & \\ \mathrm{H} & 0.7325285 & 0.3336309 & 0.2035149 \\ \mathrm{H} & -1.0276470 & 0.4269124 & 0.4608130 \\ \mathrm{C} & -0.2289275 & -0.1713185 & -0.0128015 \\ \mathrm{~N} & -0.2600378 & -1.4857612 & 0.5643845 \\ \mathrm{C} & -1.4160804 & -2.0029815 & 1.1270826 \\ \mathrm{H} & -0.0990309 & -2.7780345 & 0.5941408 \\ \mathrm{O} & -1.1214278 & -3.3185008 & 1.1968707 \\ \mathrm{O} & -2.4585364 & -1.4301959 & 1.4824076 \\ \mathrm{H} & 0.6066255 & 1.7305255 & -1.9174895 \\ \mathrm{H} & -1.1388889 & 1.9131760 & -1.6953063 \\ \mathrm{C} & -0.3662988 & 1.2673932 & -2.1321002 \\ \mathrm{C} & -0.4413708 & -0.1462244 & -1.5419754\end{array}$




$\begin{array}{rrrr}\mathrm{H} & 0.3154998 & -0.7902065 & -2.0114335 \\ \mathrm{H} & -1.4183445 & -0.5957578 & -1.7589210 \\ \mathrm{H} & -0.5039711 & 1.2703170 & -3.2216727\end{array}$

$\mathrm{TS}(13+2 \longrightarrow$ 14)

56

Energy $=-1187.201767461$

$\begin{array}{lrrr}\mathrm{H} & -4.6097895 & -2.9727376 & -3.3000767 \\ \mathrm{H} & -3.4238421 & -0.6735848 & -6.1000559 \\ \mathrm{H} & -5.1800177 & -3.6076425 & -1.7414589 \\ \mathrm{C} & -4.2871281 & -3.4348460 & -2.3574022 \\ \mathrm{H} & -6.2781964 & -4.0958093 & 1.8341969 \\ \mathrm{C} & -2.4633704 & -1.2061209 & -6.1790229 \\ \mathrm{H} & -3.8044874 & -4.3985035 & -2.5742026 \\ \mathrm{H} & -3.2467559 & -0.9254584 & -3.5079245 \\ \mathrm{H} & -2.4513483 & -1.7350172 & -7.1425169 \\ \mathrm{H} & -4.6700702 & -2.6054903 & 0.4156393 \\ \mathrm{C} & -5.4108968 & -4.7287044 & 2.0779975 \\ \mathrm{O} & -3.9076603 & -1.3134211 & -1.3420355 \\ \mathrm{H} & -3.0630542 & -2.9361166 & -5.0160953 \\ \mathrm{H} & -1.6645212 & -0.4488396 & -6.2022735 \\ \mathrm{C} & -2.2702158 & -2.1720216 & -5.0089896 \\ \mathrm{H} & -5.7793535 & -5.7488688 & 2.2559962 \\ \mathrm{H} & -4.8123648 & -5.0910331 & 0.0249623 \\ \mathrm{C} & -2.2881296 & -1.4365741 & -3.6664299 \\ \mathrm{C} & -3.3332567 & -2.4680150 & -1.6183094 \\ \mathrm{C} & -4.3729368 & -4.6981469 & 0.9549952 \\ \mathrm{C} & -3.8537912 & -3.2788165 & 0.7076014 \\ \mathrm{O} & -3.4376373 & 1.1927885 & -0.6815400 \\ \mathrm{H} & -4.9833473 & -4.3572609 & 3.0221510 \\ \mathrm{C} & -2.6012664 & 0.3534479 & -0.5234032 \\ \mathrm{H} & -1.4997415 & -0.6681549 & -3.6595073 \\ & -2.60\end{array}$




\begin{tabular}{lrrr}
$\mathrm{N}$ & -2.0986700 & -2.3056486 & -2.5058080 \\
$\mathrm{~N}$ & -2.8571724 & -3.1888218 & -0.3578985 \\
$\mathrm{H}$ & -3.4156374 & -2.8828706 & 1.6367987 \\
$\mathrm{H}$ & -1.3116865 & -2.7025768 & -5.1089640 \\
$\mathrm{~N}$ & -1.5892415 & -0.2763914 & -0.2317001 \\
$\mathrm{H}$ & -3.5211484 & -5.3473207 & 1.2068668 \\
$\mathrm{H}$ & 0.6255695 & -0.2158478 & -1.7051615 \\
$\mathrm{H}$ & -0.0219982 & 1.4283115 & -1.7091014 \\
$\mathrm{C}$ & -0.8286957 & -2.6573693 & -2.2133144 \\
$\mathrm{C}$ & 0.5242876 & 0.6945201 & -1.0945314 \\
$\mathrm{C}$ & -1.5915505 & -3.5314029 & -0.0394403 \\
$\mathrm{C}$ & -0.3160972 & 0.3599210 & 0.1385078 \\
$\mathrm{O}$ & 0.1417202 & -2.3840738 & -2.9515773 \\
$\mathrm{~N}$ & -0.6184893 & -3.3776780 & -1.0328232 \\
$\mathrm{H}$ & 0.2302451 & -0.3539448 & 0.7711097 \\
$\mathrm{O}$ & -1.2698050 & -3.9958497 & 1.0752773 \\
$\mathrm{H}$ & 2.4934306 & 1.4805692 & -1.6080300 \\
$\mathrm{C}$ & 1.9003832 & 1.2425069 & -0.7139666 \\
$\mathrm{H}$ & -0.4895560 & 1.2704518 & 0.7379707 \\
$\mathrm{H}$ & 2.4676943 & 0.5083274 & -0.1215771 \\
$\mathrm{C}$ & 0.7402863 & -3.8438443 & -0.7425175 \\
$\mathrm{H}$ & 1.8145063 & 2.1604463 & -0.1127837 \\
$\mathrm{H}$ & 0.6457809 & -4.7518855 & -0.1340851 \\
$\mathrm{H}$ & 1.2008092 & -4.1072288 & -1.7027640 \\
$\mathrm{H}$ & 1.1065779 & -2.5408877 & 0.9279102 \\
$\mathrm{C}$ & 1.6045301 & -2.8143507 & -0.0144582 \\
$\mathrm{H}$ & 1.6590721 & -1.9048829 & -0.6300246 \\
$\mathrm{C}$ & 3.0094460 & -3.3483443 & 0.2637641 \\
$\mathrm{H}$ & 2.9743055 & -4.2521239 & 0.8913239 \\
& 3.5259265 & -3.6116985 & -0.6720755 \\
\hline & 3.6234030 & -2.6013887 & 0.7861800 \\
& & &
\end{tabular}




\begin{tabular}{|c|c|c|c|}
\hline \multicolumn{4}{|c|}{$\mathrm{TS}\left(15+12 \longrightarrow 16+17+12+\mathrm{H}^{+}\right)$} \\
\hline \multicolumn{4}{|c|}{74} \\
\hline \multicolumn{4}{|c|}{ Energy $=-1514.550164990$} \\
\hline $\mathrm{H}$ & -0.4521110 & -5.1139502 & 4.1461197 \\
\hline $\mathrm{H}$ & -1.5994161 & -6.1694487 & 3.2933062 \\
\hline & -0.6192014 & -5.6791851 & 3.2162447 \\
\hline 14 & 0. 1461244 & -6.4685224 & 3.1625666 \\
\hline 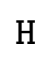 & -1.3457055 & -4.0032990 & 2.0467975 \\
\hline $\mathrm{H}$ & 0.9396767 & -3.3845762 & 2.7251060 \\
\hline C & -0.5593001 & -4.7720016 & 1.9880918 \\
\hline & 0.7934266 & -4.0708858 & 1.8822764 \\
\hline 11 & -0.7554540 & -5.3436306 & 1.0671636 \\
\hline 0 & -0.0365444 & -1.5018525 & 1.7563315 \\
\hline & 1.6233100 & -4.7858893 & 1.8948606 \\
\hline U & -1.2555842 & -8.5581939 & 0.3993828 \\
\hline 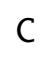 & 0.4710142 & -1.9561456 & 0.7318018 \\
\hline $\mathbb{N}$ & 0.9264065 & -3.2633361 & 0.6545814 \\
\hline & -0.3850957 & 2.6921422 & 0.6734358 \\
\hline 2 & 1.3975212 & 0.9333618 & 1.0405737 \\
\hline 1 & -0.8425304 & 0.1591315 & 0.2522066 \\
\hline $\mathrm{H}$ & -3.4375814 & -4.9271666 & -0.8645366 \\
\hline 1 & 1.6830290 & -5.8204197 & 0.2256678 \\
\hline $1+$ & -1.6252490 & -3.2702649 & -0.4471358 \\
\hline 14 & -3.8434339 & -2.3603645 & -1.2694347 \\
\hline n & 5.4450188 & -0.9234824 & 1.7491324 \\
\hline- & -0.9783264 & -8.1309573 & -0.5767818 \\
\hline 0 & -3.2360475 & -7.4814717 & -1.0659428 \\
\hline$U$ & 0.0324344 & -7.7107134 & -0.5079808 \\
\hline 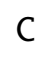 & -2.0150874 & -7.0862791 & -0.9839526 \\
\hline 1 & 1.2275988 & 3.3440639 & 0.3121841 \\
\hline & 0.4791053 & 2.6038286 & -0.0023039 \\
\hline & 6.8990590 & -1.9358802 & 1.8161724 \\
\hline
\end{tabular}




\begin{tabular}{|c|c|c|c|}
\hline & 1.0587678 & 1.1892832 & 485 \\
\hline & 0.0215792 & Q. 1593589 & -0.4221704 \\
\hline & 0.5526503 & .2117298 & -0.4 \\
\hline & 3.3203440 & -1.6142453 & 0.5602938 \\
\hline & -1.5540462 & -5.8659679 & -1.1869193 \\
\hline & 3.9296978 & -2.6997074 & 0.5076227 \\
\hline & -2.5541633 & -4.8689685 & -1.5342808 \\
\hline 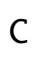 & 1.6019933 & -3.7836554 & -0.4386907 \\
\hline C & 3.4157383 & -3.8530661 & 0.1155541 \\
\hline & 5.2468180 & -2.8080837 & 0.9086490 \\
\hline & 0.3969449 & -5.4534441 & $-\theta$. \\
\hline 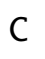 & 1.4611914 & -5.2511829 & -0.6799801 \\
\hline 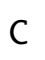 & 6.0596858 & -1.6300828 & 1.1722852 \\
\hline $\mathrm{H}$ & 5.7219625 & -3.6728448 & 0.6676944 \\
\hline C & -1.9669531 & -3.4588143 & -1.475 \\
\hline & -0.9709723 & -8.9615135 & -1.3005608 \\
\hline 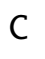 & -2.9465429 & -2.3699808 & -1.9083694 \\
\hline F & -2.4888067 & -1.3711717 & -1.8531212 \\
\hline & 0.1425043 & 2.8743482 & -1.0145723 \\
\hline$\Pi$ & 1.9 & 1. & -0.6332 \\
\hline H & -0.3191697 & 0.3742069 & -1.4415868 \\
\hline & -2.9594961 & -5.0431379 & -2.5553620 \\
\hline $\mathrm{F}$ & 2.1419994 & -5.5912648 & -1.4637047 \\
\hline $\mathrm{H}$ & 6.8096576 & 1.0504691 & 0.7788964 \\
\hline 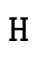 & -1.0690003 & -3.4299177 & -2.1126677 \\
\hline$C$ & 1.1849175 & -1.6552849 & -1.5823055 \\
\hline $\mathrm{N}$ & 1.6322444 & -2.9686119 & -1.5626773 \\
\hline H & -3.2810007 & -2.5246940 & -2.9461676 \\
\hline & 8.2761604 & 0.0531390 & 0.8709052 \\
\hline C & 6.5857118 & -0.9385892 & -0.0895075 \\
\hline$C$ & 7.4120282 & 0.3058248 & 0.2368615 \\
\hline $\mathrm{H}$ & 5.7220147 & -0.6707387 & -0.7186341 \\
\hline 0 & 1.2989491 & -0.9445084 & -2.58004 \\
\hline
\end{tabular}




$\begin{array}{llll}\mathrm{H} & 7.1925362 & -1.6564311 & -0.6661140 \\ \mathrm{H} & 3.0563930 & -4.1792932 & -2.4835834 \\ \mathrm{C} & 2.3060321 & -3.4417266 & -2.7871070 \\ \mathrm{H} & 0.7306085 & -4.8049712 & -3.3574142 \\ \mathrm{H} & 7.7959041 & 0.7825143 & -0.6760832 \\ \mathrm{H} & 2.8402891 & -2.5792293 & -3.2026500 \\ \mathrm{C} & 1.3336758 & -4.0090307 & -3.8201241 \\ \mathrm{H} & 0.6342606 & -3.2143974 & -4.1193016 \\ \mathrm{H} & 2.7620651 & -5.3642916 & -4.7580150 \\ \mathrm{C} & 2.0802882 & -4.5480427 & -5.0406734 \\ \mathrm{H} & 1.3797654 & -4.9386522 & -5.7910178 \\ \mathrm{H} & 2.6809125 & -3.7598004 & -5.5190703\end{array}$

$$
\mathrm{TS}(17+2 \longrightarrow \text { 18) }
$$

28

\begin{tabular}{lrrr}
\multicolumn{4}{l}{ Energy $=-649.5127014818$} \\
$\mathrm{H}$ & 2.0339340 & -0.2492815 & -2.4239238 \\
$\mathrm{C}$ & 1.0201901 & 0.1143413 & -2.6405066 \\
$\mathrm{H}$ & 0.7951765 & -0.1267889 & -3.6871156 \\
$\mathrm{H}$ & 1.0328300 & 1.2067459 & -2.5435040 \\
$\mathrm{H}$ & -2.1818417 & 1.3870995 & 4.3189151 \\
$\mathrm{H}$ & 1.2898107 & -0.5766044 & 0.0589702 \\
$\mathrm{C}$ & -0.0075091 & -0.4970770 & -1.6822140 \\
$\mathrm{H}$ & -0.0384920 & -1.5885539 & -1.8162502 \\
$\mathrm{H}$ & -2.6258429 & 3.5364662 & 5.5607605 \\
$\mathrm{H}$ & -1.3549002 & 2.6165629 & 6.3832770 \\
$\mathrm{C}$ & 0.3024387 & -0.1710010 & -0.2133615 \\
$\mathrm{C}$ & -1.6062522 & 3.1481991 & 5.4561914 \\
$\mathrm{C}$ & -1.4854910 & 2.2293483 & 4.2366449 \\
$\mathrm{H}$ & 0.3276388 & 0.9150713 & -0.0829841 \\
$\mathrm{H}$ & 0.2185002 & 1.0857376 & 4.9514340 \\
$\mathrm{H}$ & -1.0074000 & -0.1070869 & -1.8992664
\end{tabular}




$\begin{array}{lrrr}\mathrm{H} & -1.7575424 & 2.7596910 & 3.3178394 \\ \mathrm{C} & -0.0667400 & 1.6744415 & 4.0634628 \\ \mathrm{~N} & 0.0171969 & 0.8936059 & 2.8323184 \\ \mathrm{C} & 0.0440124 & -0.3414879 & 2.8290465 \\ \mathrm{~N} & -0.6868198 & -0.6654006 & 0.7347362 \\ \mathrm{O} & 0.2326771 & -1.4719836 & 3.1693372 \\ \mathrm{H} & -0.7655177 & -1.6794361 & 0.7731837 \\ \mathrm{H} & -0.9284131 & 4.0079702 & 5.3738255 \\ \mathrm{H} & 0.6417434 & 2.5108076 & 3.9811931 \\ \mathrm{C} & -2.0587561 & -0.0527089 & 0.6780777 \\ \mathrm{O} & -2.1060740 & 1.0695873 & 0.1266775 \\ \mathrm{O} & -2.9321385 & -0.7617379 & 1.2230571\end{array}$

\subsubsection{Deprotonated Urea Catalysis}

\section{Compound 18}

\begin{tabular}{lrrr}
25 & & \\
\multicolumn{4}{l}{ Energy $=-460.8839624575$} \\
$\mathrm{H}$ & -1.1625213 & -3.8060353 & 1.0047359 \\
$\mathrm{H}$ & -1.6360896 & -1.2742675 & 0.6421503 \\
$\mathrm{C}$ & -0.1206027 & -3.5327447 & 1.2324401 \\
$\mathrm{H}$ & 0.2081350 & -2.4084362 & -0.5960260 \\
$\mathrm{C}$ & -0.5845229 & -1.0523964 & 0.8764310 \\
$\mathrm{H}$ & 0.5173686 & -4.3835786 & 0.9533429 \\
$\mathrm{C}$ & 0.2855881 & -2.2566851 & 0.4928334 \\
$\mathrm{H}$ & -0.1549950 & 0.2513093 & -3.8416042 \\
$\mathrm{H}$ & 0.5789973 & 1.3599928 & -6.0395199 \\
$\mathrm{~N}$ & -0.2421848 & 0.1901570 & 0.1890788 \\
$\mathrm{O}$ & -1.6184807 & -0.2892312 & -1.6116327 \\
$\mathrm{H}$ & 1.9491983 & 1.6274821 & -3.9228952 \\
$\mathrm{C}$ & -0.5528593 & 0.2848692 & -1.2059997 \\
$\mathrm{H}$ & 0.7249292 & 0.4757497 & 0.3490657 \\
$\mathrm{H}$ & -0.0430662 & -3.4001858 & 2.3228705
\end{tabular}




$\begin{array}{lrrr}\mathrm{N} & 0.3020501 & 1.0200332 & -1.9053790 \\ \mathrm{H} & -0.5175070 & -0.8804426 & 1.9639006 \\ \mathrm{C} & -0.0688541 & 1.2172490 & -3.2960697 \\ \mathrm{C} & 0.9541560 & 2.0948357 & -4.0184884 \\ \mathrm{C} & 0.6197861 & 2.3163209 & -5.4947719 \\ \mathrm{H} & 1.3686147 & 2.9509564 & -5.9908003 \\ \mathrm{H} & 1.3414521 & -2.0182655 & 0.7065602 \\ \mathrm{H} & -1.0734675 & 1.6855957 & -3.3957050 \\ \mathrm{H} & -0.3607594 & 2.8041433 & -5.6106373 \\ \mathrm{H} & 1.0194485 & 3.0648577 & -3.4963998\end{array}$

\section{Compound $18+\mathrm{H}^{+}$}

\begin{tabular}{lrrr} 
Ln & \multicolumn{3}{l}{} \\
Energy $=-461.3895322453$ & \\
$\mathrm{H}$ & 0.6719043 & -3.8034067 & 1.5628472 \\
$\mathrm{H}$ & -0.7096833 & -1.8934053 & 0.4804405 \\
$\mathrm{C}$ & 1.3665913 & -3.0360047 & 1.9372226 \\
$\mathrm{H}$ & 1.7516346 & -2.1157239 & 0.0093841 \\
$\mathrm{C}$ & 0.0160963 & -1.1666173 & 0.8752012 \\
$\mathrm{H}$ & 2.3605782 & -3.4967295 & 2.0229836 \\
$\mathrm{C}$ & 1.3960428 & -1.8200833 & 1.0099365 \\
$\mathrm{H}$ & 1.3346802 & 3.6584473 & 0.7438804 \\
$\mathrm{H}$ & 0.1301249 & 5.8965271 & 1.2550493 \\
$\mathrm{~N}$ & -0.0015030 & -0.0004894 & 0.0004028 \\
$\mathrm{O}$ & 0.5299542 & 1.4857858 & 1.6720043 \\
$\mathrm{H}$ & -1.1651847 & 3.7847641 & 0.7374965 \\
$\mathrm{C}$ & 0.4014002 & 1.2326390 & 0.4585145 \\
$\mathrm{H}$ & 0.0881510 & -0.1804260 & -0.9962394 \\
$\mathrm{H}$ & 1.0384598 & -2.7536272 & 2.9491572 \\
$\mathrm{~N}$ & 0.6519709 & 2.1659682 & -0.5206166 \\
$\mathrm{H}$ & -0.3424456 & -0.8432903 & 1.8622188 \\
$\mathrm{C}$ & 0.7662572 & 3.5820002 & -0.1933552
\end{tabular}




$\begin{array}{lrrr}\text { C } & -0.5819860 & 4.2957802 & -0.0448551 \\ \text { C } & -0.4117730 & 5.7749864 & 0.3047850 \\ \text { H } & 0.3570696 & 1.9413726 & -1.4673503 \\ \text { H } & -1.3846772 & 6.2757022 & 0.4065701 \\ \text { H } & 2.1047264 & -1.0680551 & 1.3913738 \\ \text { H } & 1.3634568 & 4.0618199 & -0.9833130 \\ \text { H } & 0.1581594 & 6.3036575 & -0.4745379 \\ \text { H } & -1.1482747 & 4.1917267 & -0.9847782\end{array}$

\section{Compound 19}

$\begin{array}{lrrr}l & & \\ \text { Energy }=-649.6260676224 & \\ \mathrm{H} & -2.1121836 & 0.1836165 & -0.0731494 \\ \mathrm{H} & -1.6740382 & -0.3939094 & -2.4985660 \\ \mathrm{C} & -1.2121015 & 0.5550332 & -0.5883324 \\ \mathrm{C} & -1.4547138 & 0.6076395 & -2.0974244 \\ \mathrm{H} & -0.2257979 & -1.3709037 & -0.5835388 \\ \mathrm{H} & -2.3034007 & 1.2607954 & -2.3450738 \\ \mathrm{C} & 1.1157194 & 0.5679184 & 1.7856078 \\ \mathrm{O} & 1.5971264 & 1.4050586 & 0.9804187 \\ \mathrm{~N} & 0.2223411 & -0.4197577 & 1.2140124 \\ \mathrm{O} & 1.3505296 & 0.5138342 & 3.0365490 \\ \mathrm{~N} & -0.3322232 & -1.4385661 & 3.2612411 \\ \mathrm{C} & -0.0319532 & -0.3503991 & -0.2304108 \\ \mathrm{C} & -0.4854224 & -1.4189286 & 1.9155205 \\ \mathrm{H} & 0.3727025 & -0.7625906 & 3.6052885 \\ \mathrm{H} & -1.0355507 & -2.1050165 & 5.0920132 \\ \mathrm{O} & -1.2228345 & -2.2206279 & 1.3030721 \\ \mathrm{C} & -0.8723683 & -2.5079781 & 4.0806456 \\ \mathrm{H} & -1.8541304 & -2.7960155 & 3.6763039 \\ \mathrm{H} & -1.0100095 & 1.5657872 & -0.2003919 \\ \mathrm{H} & -0.5697257 & 0.9918943 & -2.6277265\end{array}$




$\begin{array}{lrrr}\mathrm{H} & 0.8830807 & 0.0240012 & -0.7030994 \\ \mathrm{C} & 0.0359953 & -3.7420615 & 4.1540264 \\ \mathrm{H} & -0.7088340 & -4.4820507 & 6.0671971 \\ \mathrm{H} & 1.0221773 & -3.4352909 & 4.5385088 \\ \mathrm{C} & -0.5555625 & -4.8409162 & 5.0377956 \\ \mathrm{H} & -1.5308380 & -5.1763067 & 4.6530608 \\ \mathrm{H} & 0.1957970 & -4.1197720 & 3.1317650 \\ \mathrm{H} & 0.1062287 & -5.7171725 & 5.0835817\end{array}$

\section{Compound 20}

$\begin{array}{lrrr}38 & & \\ \text { Energy }=-747.6987600081 & \\ \mathrm{H} & 4.8115438 & -2.3066468 & -3.4560923 \\ \mathrm{H} & 3.7573834 & -0.6492143 & -1.7747043 \\ \mathrm{O} & 1.5014032 & 0.4005677 & -2.2057213 \\ \mathrm{H} & -1.8510589 & 0.7872039 & -0.7497382 \\ \mathrm{C} & 4.3693867 & -3.1236700 & -2.8654142 \\ \mathrm{C} & 3.3259993 & -1.4936086 & -1.2146064 \\ \mathrm{C} & 1.2662614 & -0.1750808 & -1.1154793 \\ \mathrm{H} & -0.7112491 & 1.0082818 & -2.1094575 \\ \mathrm{H} & 5.1220494 & -3.4478985 & -2.1302648 \\ \mathrm{H} & -0.1857502 & -1.1782890 & 3.4096257 \\ \mathrm{C} & -0.8261816 & 1.0672048 & -1.0198513 \\ \mathrm{H} & 4.0582667 & -1.7888178 & -0.4463089 \\ \mathrm{H} & 4.1817593 & -3.9659504 & -3.5462524 \\ \mathrm{H} & 0.4524821 & -2.8146218 & 5.2993074 \\ \mathrm{C} & 3.0844215 & -2.6675615 & -2.1730815 \\ \mathrm{~N} & 0.0675447 & 0.0718871 & -0.4226748 \\ \mathrm{~N} & 2.1274581 & -1.0432243 & -0.5355938 \\ \mathrm{H} & 1.9989057 & -2.4213196 & 3.3276528 \\ \mathrm{H} & -2.5394395 & 3.2811007 & -0.9352526 \\ \mathrm{~N} & 0.4619993 & -1.3913133 & 1.3971543\end{array}$




$\begin{array}{lrrr}\text { H } & 2.3379700 & -2.3572006 & -2.9212956 \\ \text { C } & -0.3598111 & -0.5126931 & 0.8428656 \\ \text { C } & 0.0070502 & -1.9654095 & 2.6505715 \\ \text { O } & -1.4827349 & -0.1231282 & 1.2838737 \\ \text { C } & 1.0391392 & -2.9496230 & 3.2010049 \\ \text { H } & -1.4010469 & 3.5020264 & -2.2810183 \\ \text { C } & -1.4914221 & 3.5096957 & -1.1836794 \\ \mathrm{C} & 0.6092894 & -3.5822709 & 4.5254798 \\ \text { H } & 1.7389855 & -1.4729310 & 0.3523837 \\ \mathrm{C} & -0.5415466 & 2.4946048 & -0.5462320 \\ \text { H } & 1.3654937 & -4.2861390 & 4.9019203 \\ \text { H } & 2.6402019 & -3.5025401 & -1.6071935 \\ \text { H } & -0.9641677 & -2.4894950 & 2.5278721 \\ \text { H } & -0.6348078 & 2.5240651 & 0.5509168 \\ \text { H } & -0.3358580 & -4.1358953 & 4.4123883 \\ \text { H } & -1.2806734 & 4.5316576 & -0.8377734 \\ \text { H } & 1.2173159 & -3.7340400 & 2.4464537 \\ \text { H } & 0.5026252 & 2.7474351 & -0.7901926\end{array}$

\section{Compound $20+\mathrm{H}^{+}$}

39

Energy $=-748.1914951386$

$\begin{array}{lrrr}\mathrm{H} & -3.6790296 & 4.0115172 & -2.5733459 \\ \mathrm{H} & -2.6605754 & 1.7010278 & -2.3945570 \\ \mathrm{H} & 4.0521982 & -1.9841582 & 0.1516920 \\ \mathrm{H} & -3.6280417 & 3.2170587 & -4.1622117 \\ \mathrm{C} & -3.0058551 & 3.6199469 & -3.3510281 \\ \mathrm{C} & -2.0743306 & 2.5485737 & -2.7829398 \\ \mathrm{H} & 5.1836022 & -3.3315885 & -1.7584311 \\ \mathrm{C} & 3.4416509 & -1.4702869 & -0.6043318 \\ \mathrm{H} & 3.9767170 & -0.5600621 & -0.9060954 \\ \mathrm{H} & 1.7886471 & -1.7177074 & 0.7026355\end{array}$




\begin{tabular}{lrrr}
$\mathrm{H}$ & -1.8291844 & 3.4800967 & -0.8391217 \\
$\mathrm{~N}$ & 2.1991235 & -1.0663231 & 0.0453717 \\
$\mathrm{C}$ & -1.1963547 & 3.0953849 & -1.6515546 \\
$\mathrm{C}$ & 4.5422178 & -2.7881099 & -2.4687908 \\
$\mathrm{H}$ & -1.7677082 & -1.6274847 & 0.2193040 \\
$\mathrm{H}$ & 5.1007304 & -1.9061334 & -2.8171074 \\
$\mathrm{H}$ & -2.4335926 & 4.4685105 & -3.7560677 \\
$\mathrm{H}$ & -0.2304890 & -2.4906568 & 0.1485072 \\
$\mathrm{~N}$ & -0.2891780 & 2.1018123 & -1.0964448 \\
$\mathrm{H}$ & -1.4236445 & 2.1499277 & -3.5776645 \\
$\mathrm{C}$ & 3.2205439 & -2.3797164 & -1.8171141 \\
$\mathrm{C}$ & -0.8990071 & -1.9317886 & 0.8225515 \\
$\mathrm{O}$ & -1.7835546 & 1.2968834 & 0.4331818 \\
$\mathrm{C}$ & -0.6822012 & 1.2411721 & -0.1359499 \\
$\mathrm{C}$ & 1.4809075 & 0.0000306 & -0.3950925 \\
$\mathrm{H}$ & -1.8527716 & -3.7271202 & 1.6085025 \\
$\mathrm{~N}$ & $\mathbb{0 . 2 5 3 6 7 1 1}$ & 0.2271100 & 0.2482211 \\
$\mathrm{H}$ & 2.6631940 & -3.2742500 & -1.4959234 \\
$\mathrm{H}$ & $\mathbb{0 . 6 2 6 9 0 1 8}$ & 1.9060995 & -1.5188504 \\
$\mathrm{H}$ & 4.3716451 & -3.4417717 & -3.3352458 \\
$\mathrm{H}$ & -0.5823591 & 3.9301279 & -2.0201711 \\
$\mathrm{C}$ & -1.3428260 & -2.8276487 & 1.9792781 \\
$\mathrm{O}$ & 1.9195000 & 0.7347672 & -1.3061953 \\
$\mathrm{C}$ & -0.2030028 & -0.6654116 & 1.3286979 \\
$\mathrm{H}$ & 2.5849793 & -1.8497216 & -2.5433578 \\
$\mathrm{H}$ & -2.0377253 & -2.2958230 & 2.6463235 \\
& -0.4819097 & -3.1530725 & 2.5826084 \\
$\mathrm{H}$ & -0.9035095 & -0.0823516 & 1.9355005 \\
\hline & 0.6530348 & -0.9007777 & 1.9772887
\end{tabular}

\section{Compound 21}

51 


\begin{tabular}{|c|c|c|c|}
\hline \multicolumn{4}{|c|}{ Energy $=-1034.490431581$} \\
\hline $\mathrm{H}$ & 1.1131453 & 0.6549040 & -5.4888544 \\
\hline $\mathrm{H}$ & 2.3503489 & -0.3994072 & -7.4924338 \\
\hline C & 2.0582284 & 1.1068970 & -5.1470305 \\
\hline & 3.2756272 & 0.0672064 & -7.1204727 \\
\hline 10 & 2.1349597 & 2.1002251 & -5.6135590 \\
\hline n & 3.1579909 & -0.7505293 & -5.1145647 \\
\hline H & 3.3830022 & 1.0405608 & -7.6234630 \\
\hline H & 1.8912873 & 0.3937180 & -3.1207934 \\
\hline 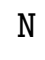 & 1.9724947 & 1.2816734 & -3.7038255 \\
\hline 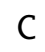 & 3.2351635 & 0.2348203 & -5.6012500 \\
\hline 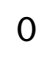 & 3.5162599 & 3.0117472 & -3.6877965 \\
\hline $\mathrm{I}$ & 4.1183860 & -0.5659663 & -7.4318967 \\
\hline 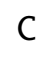 & 2.7516954 & 2.2238747 & -3.0964465 \\
\hline & 0.4834470 & 2.8838696 & -1.0007221 \\
\hline v & 2.7418382 & -0.6750795 & 0.3394308 \\
\hline 1 & 3.7185155 & 4.0257413 & -1.5219740 \\
\hline 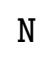 & 2.6157824 & 2.2435574 & -1.7050560 \\
\hline 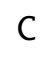 & 1.3244929 & 1.9749682 & -1.1070711 \\
\hline- & 3.4979035 & 3.1249036 & -0.9295751 \\
\hline 1 & 2.9473013 & 3.4433058 & -0.0326425 \\
\hline & 4.1692106 & 0.6933231 & -5.2414536 \\
\hline 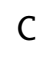 & 2.1711335 & -0.3457194 & -0.7337582 \\
\hline 1 & 3.0755219 & -2.3635189 & -3.0929684 \\
\hline N & 1.1544254 & 0.7334246 & -0.6193013 \\
\hline $\mathrm{H}$ & 3.3284517 & -2.5577340 & -1.3422250 \\
\hline . & 0.1524721 & -0.3623248 & 0.8244547 \\
\hline 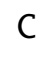 & 3.3060374 & -1.8149770 & -2.1633471 \\
\hline 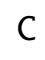 & 4.7947303 & 2.4186368 & -0.5324860 \\
\hline $\mathrm{H}$ & 5.9899805 & 4.2278857 & -0.2772634 \\
\hline J & 2.2568425 & -0.8240650 & -1.9515045 \\
\hline & -0.0966099 & 0.3845228 & 0.0566551 \\
\hline & 5.3052493 & 2.0855542 & -1.4493511 \\
\hline
\end{tabular}




$\begin{array}{lrrr}\text { C } & 5.7139763 & 3.3241383 & 0.2875979 \\ \text { H } & 5.5694467 & -2.7227592 & -3.5692775 \\ \text { H } & 5.2236659 & 3.6497425 & 1.2180067 \\ \text { H } & 5.8530789 & -2.9781441 & -1.8344707 \\ \text { H } & -0.4766043 & 1.2829309 & 0.5624517 \\ \text { H } & 4.5378311 & 1.5090051 & 0.0324712 \\ \text { C } & 5.7839339 & -2.2086732 & -2.6191983 \\ \text { H } & -0.7181975 & -1.0294451 & -1.4425507 \\ \mathrm{C} & 4.6989346 & -1.1824773 & -2.2924763 \\ \mathrm{C} & -1.1469687 & -0.1658515 & -0.9114110 \\ \text { H } & -2.2380178 & -1.3452777 & 0.5650400 \\ \text { H } & 6.6427768 & 2.8047455 & 0.5625376 \\ \text { H } & 4.6600083 & -0.4081666 & -3.0740753 \\ \text { H } & 4.9316309 & -0.6628029 & -1.3508959 \\ \mathrm{C} & -2.4326882 & -0.5653226 & -0.1869291 \\ \text { H } & 6.7725777 & -1.7358372 & -2.7113801 \\ \text { H } & -1.3590274 & 0.6012339 & -1.6724382 \\ \mathrm{H} & -2.8793353 & 0.2957748 & 0.3332512 \\ \mathrm{H} & -3.1803262 & -0.9567535 & -0.8908097\end{array}$

\section{Compound $21+\mathrm{H}^{+}$}

52

Energy $=-1034.981523241$

$\begin{array}{lrrr}\mathrm{H} & 1.8105305 & -3.4781975 & -1.2181386 \\ \mathrm{C} & 2.0016636 & -2.5289683 & -1.7410206 \\ \mathrm{H} & 3.0777585 & -2.4735632 & -1.9552221 \\ \mathrm{H} & 1.4674910 & -2.5618578 & -2.7024507 \\ \mathrm{C} & 1.5467710 & -1.3360883 & -0.8996877 \\ \mathrm{H} & -0.1914320 & -2.3198974 & -0.0426835 \\ \mathrm{C} & 0.0478009 & -1.3983125 & -0.5912521 \\ \mathrm{H} & 2.1073798 & -1.3053009 & 0.0479439 \\ \mathrm{H} & -0.5328139 & -1.4021305 & -1.5234399\end{array}$




$\begin{array}{lrrr}\mathrm{H} & 1.7617371 & -0.3949271 & -1.4293833 \\ \mathrm{~N} & -0.4209833 & -0.2797511 & 0.2208988 \\ \mathrm{H} & 1.1629894 & -0.0398119 & 5.8037963 \\ \mathrm{H} & 2.2073120 & 0.6615249 & 8.0721668 \\ \mathrm{H} & -0.2937757 & -0.3372284 & 1.2264694 \\ \mathrm{H} & -0.6711207 & 1.7742959 & 4.2949385 \\ \mathrm{H} & 2.7985507 & 3.1976843 & 2.4238613 \\ \mathrm{O} & -0.9447145 & 1.0777288 & -1.5382426 \\ \mathrm{H} & 2.1133224 & 2.2573121 & 6.1102685 \\ \mathrm{C} & -0.7861827 & 0.9014965 & -0.3257939 \\ \mathrm{C} & 0.5104242 & 0.8430256 & 5.8501042 \\ \mathrm{~N} & 0.1734459 & 1.2316133 & 4.4863767 \\ \mathrm{C} & 1.5281246 & 1.5275072 & 8.0699862 \\ \mathrm{C} & 1.1956102 & 1.9619180 & 6.6421288 \\ \mathrm{H} & 4.0373584 & 4.2954298 & 0.5262552 \\ \mathrm{C} & 2.4165040 & 3.1261674 & 1.3943833 \\ \mathrm{H} & 1.6655597 & 3.9232629 & 1.2726025 \\ \mathrm{H} & 2.0159883 & 2.3386675 & 8.6279101 \\ \mathrm{C} & 3.5556093 & 3.3174496 & 0.3923513 \\ \mathrm{H} & -0.4243024 & 0.5439616 & 6.3458560 \\ \mathrm{H} & -3.7111225 & 2.0377252 & 1.3396385 \\ \mathrm{H} & \mathbb{0} .6185816 & 1.2409418 & 8.6195404 \\ \mathrm{~N} & 0.6406058 & 1.5698823 & 2.1827772 \\ \mathrm{H} & 4.3257863 & 2.5416730 & 0.5164312 \\ \mathrm{C} & 1.0753704 & 1.1488267 & 3.4883111 \\ \mathrm{H} & -1.0689386 & 1.9646090 & 0.5906045 \\ \mathrm{C} & -0.6493204 & 2.0437779 & 1.9152250 \\ \mathrm{H} & 1.7358553 & 1.7715916 & 1.2050362 \\ \mathrm{H} & 0.5356478 & 2.8439884 & 6.6568377 \\ \mathrm{H} & 2.4492845 & 0.9518704 & 1.3389107 \\ \mathrm{H} & -2.1284144 & 2.9015149 & 0.1648175\end{array}$




$\begin{array}{lrrr}\mathrm{O} & 2.2185403 & 0.6938119 & 3.6185816 \\ \mathrm{H} & -2.0274798 & 3.8020532 & 0.7815811 \\ \mathrm{C} & -3.5338603 & 2.3148327 & 0.2898293 \\ \mathrm{H} & 1.3317855 & 1.7003290 & 0.1925776 \\ \mathrm{H} & -3.5896476 & 1.3885286 & -0.3038024 \\ \mathrm{H} & -1.9123239 & 3.1684958 & -0.8772342 \\ \mathrm{C} & -4.5975909 & 3.3044496 & -0.1871208 \\ \mathrm{H} & -4.5716648 & 4.2308473 & 0.4063066 \\ \mathrm{H} & -5.6048037 & 2.8752677 & -0.0963747 \\ \mathrm{H} & -4.4409814 & 3.5771596 & -1.2416854\end{array}$

\section{Compound 22}

\begin{tabular}{lrrr} 
64 & \multicolumn{3}{l}{} \\
Energy $=-1321.283806313$ & \\
$\mathrm{H}$ & 2.3585348 & -0.9033854 & -3.1213169 \\
$\mathrm{H}$ & 0.0004216 & -1.1530651 & -2.2546541 \\
$\mathrm{H}$ & 1.7955151 & -2.5827474 & -3.2642812 \\
$\mathrm{C}$ & 2.0482828 & -1.7896821 & -2.5474455 \\
$\mathrm{C}$ & 0.8602408 & -1.4689796 & -1.6411214 \\
$\mathrm{H}$ & 1.5596180 & 0.5282724 & -1.1716830 \\
$\mathrm{O}$ & -2.6225720 & 0.0529135 & 0.4218776 \\
$\mathrm{H}$ & -0.9278463 & -0.2086310 & -0.2225414 \\
$\mathrm{H}$ & -5.3439254 & 2.4576636 & 1.1440900 \\
$\mathrm{H}$ & -7.4013201 & 1.0669674 & 1.9596679 \\
$\mathrm{C}$ & 1.1872207 & -0.3661786 & -0.6381625 \\
$\mathrm{H}$ & 2.9149941 & -2.1286553 & -1.9604822 \\
$\mathrm{H}$ & -4.7847002 & 1.0250957 & 0.2459394 \\
$\mathrm{H}$ & 0.5421831 & -2.3706937 & -1.0941774 \\
$\mathrm{~N}$ & 0.0097051 & -0.0279069 & 0.1437674 \\
$\mathrm{O}$ & -3.9905141 & 3.9048427 & 2.4693383 \\
$\mathrm{C}$ & -4.7495755 & 1.5402554 & 1.2151724 \\
$\mathrm{C}$ & -2.3765216 & 1.0151164 & 1.1640640
\end{tabular}




\begin{tabular}{|c|c|c|c|}
\hline & -6.7283023 & 0.2005642 & 2.0474276 \\
\hline & -6.7904208 & -0.3677898 & 1.1069903 \\
\hline & -3.1440870 & 3.3214346 & 1.7690555 \\
\hline & -3.3564254 & 1.9524589 & 1.4543494 \\
\hline & 1.9877222 & -0.6880740 & 0.0438673 \\
\hline & 0.0815175 & 0.6899734 & 1.2841467 \\
\hline & -5.2922593 & 0.6445796 & 2.3278157 \\
\hline & -1.1514246 & 1.1505902 & 1.8319175 \\
\hline & -2.0428189 & 5.2223598 & 2.9506575 \\
\hline & -7.1099676 & -0.4409304 & 2.8537395 \\
\hline & -0.5612251 & 5.2436340 & 1.9485027 \\
\hline & -5.2389473 & 1.1957238 & 3.2793603 \\
\hline ( & -1.6584853 & 5.2092598 & 1.9226242 \\
\hline ( & 1.1451466 & 0.9556299 & 1.8625201 \\
\hline 1 & -2.0126040 & 3.9220765 & 1.3190626 \\
\hline & -4.6395150 & -0.2367291 & 2.4301863 \\
\hline 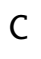 & -1.0586279 & 1.7115702 & 3.1924881 \\
\hline I & -1.9827046 & 2.2536212 & 3.4084531 \\
\hline C & -1.1861933 & 3.5166416 & 0.1477377 \\
\hline 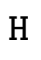 & -0.2317203 & 2.4314662 & 3.2132927 \\
\hline$F$ & -0.6904016 & 7.8285346 & 1.8019476 \\
\hline$c$ & 0.0408545 & 3.7960174 & 0.2445831 \\
\hline $\mathrm{H}$ & -2.1634987 & 7.8220982 & 2.7954918 \\
\hline C & -1.7865907 & 7.7347137 & 1.764 \\
\hline C & -2.2035032 & 6.4040900 & 1.1375681 \\
\hline $\mathrm{N}$ & -1.8719292 & 2.9595998 & -0.8174537 \\
\hline$C$ & -0.8705932 & 0.6229952 & 4.2508987 \\
\hline $\mathrm{H}$ & -3.3010536 & 6.3289777 & 1.0939069 \\
\hline $\mathrm{H}$ & -1.8354666 & 6.3426983 & 0.1006581 \\
\hline $\mathrm{H}$ & -1.7287784 & -0.0667316 & 4.2062890 \\
\hline $\mathrm{H}$ & -0.1072399 & 2.0757311 & -1.6116996 \\
\hline $\mathrm{H}$ & 0.0293526 & 0.0379123 & 4.0143315 \\
\hline & -2.1772659 & 8.5873233 & 1.191 \\
\hline
\end{tabular}




$\begin{array}{lrrr}\text { C } & -1.0640329 & 2.5236251 & -1.9456420 \\ \text { C } & -0.7536909 & 1.2297339 & 5.6498819 \\ \text { H } & -1.6512177 & 1.8125407 & 5.9075914 \\ \text { H } & -2.0743988 & 0.6433320 & -2.1892229 \\ \text { H } & -0.7733925 & 3.3895820 & -2.5792832 \\ \text { H } & 0.1132883 & 1.9042629 & 5.7161982 \\ \text { C } & -1.8191584 & 1.5162981 & -2.8113004 \\ \text { H } & -0.0549867 & 0.6422237 & -3.7526182 \\ \text { H } & -0.6307347 & 0.4478289 & 6.4118296 \\ \text { H } & -2.7808677 & 1.9605227 & -3.1173406 \\ \mathrm{C} & -1.0216852 & 1.0833292 & -4.0418270 \\ \text { H } & -0.8057731 & 1.9415025 & -4.6972605 \\ \text { H } & -1.5638065 & 0.3345197 & -4.6371848\end{array}$

\section{Compound 23}

\begin{tabular}{llll}
64 & & \\
\multicolumn{4}{l}{ Energy $=-1321.306509251$} \\
$\mathrm{H}$ & -1.2659819 & -6.9832929 & -3.2079826 \\
$\mathrm{C}$ & -0.5890729 & -6.7160009 & -2.3843692 \\
$\mathrm{H}$ & 0.4271081 & -6.6312451 & -2.7997087 \\
$\mathrm{H}$ & -0.5868368 & -7.5513605 & -1.6672065 \\
$\mathrm{H}$ & -3.3991861 & -3.2200798 & 5.6097225 \\
$\mathrm{H}$ & -2.0379489 & -5.5055382 & -1.3023670 \\
$\mathrm{C}$ & -1.0208621 & -5.4134443 & -1.7099854 \\
$\mathrm{H}$ & -1.0332952 & -4.5923679 & -2.4434909 \\
$\mathrm{H}$ & -3.0220915 & -3.6436161 & 3.0521651 \\
$\mathrm{C}$ & -2.8576829 & -2.2679872 & 5.5021547 \\
$\mathrm{H}$ & -3.6049331 & -1.4758813 & 5.3407870 \\
$\mathrm{H}$ & -3.8913355 & -6.5675267 & 7.0776595 \\
$\mathrm{H}$ & -3.3717149 & -1.9132085 & 2.8508634 \\
$\mathrm{C}$ & -2.5686473 & -2.6442348 & 3.0217008 \\
$\mathrm{H}$ & -2.3495701 & -2.0627735 & 6.4547627
\end{tabular}




\begin{tabular}{|c|c|c|c|}
\hline & -2.1500336 & -5.4597592 & 3082 \\
\hline & -3.5803740 & -5.8797749 & 4.6755391 \\
\hline & -0.0829943 & -5.0354964 & -0.5627636 \\
\hline & -2.2114735 & -4.8320789 & 1.2494291 \\
\hline & -2.7371773 & -6.3087547 & 5.2411588 \\
\hline & -1.8618358 & -2.3308493 & 4.3436356 \\
\hline & -3.2586838 & -7.1582533 & 6.4001848 \\
\hline & 0.9440725 & -4.9221247 & -0.9384252 \\
\hline & -0.0829630 & -5.8241487 & 0.2013537 \\
\hline & -1.1025153 & -3.1015266 & 4.5420205 \\
\hline & -1.9807381 & -5.9849719 & 2.4140214 \\
\hline & -1.6878824 & -2.6356151 & 1.8528225 \\
\hline & -1.2256321 & -4.0032453 & 1.3935870 \\
\hline & -0.4632608 & -3.7964122 & 0.1147868 \\
\hline & -2.4277971 & -7.5717827 & 921209 \\
\hline & -3.8597408 & -8.0044174 & 6.0330333 \\
\hline & -1.3316033 & -1.3716895 & 4.2456935 \\
\hline $\mathrm{N}$ & -1.3426065 & -6.3216961 & 3.1673939 \\
\hline 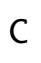 & -1.8 & -7 & 4.2775605 \\
\hline ( & -1.3557454 & -1.4355023 & 1.3198251 \\
\hline C & -1.7774896 & -0.3529223 & 1.7688164 \\
\hline$C$ & -0.0717056 & -2.6217495 & -0.4343665 \\
\hline$v$ & 0.6259459 & -2.5532917 & -1.4637777 \\
\hline IV & -0.1626254 & -4.4414612 & 2.4137917 \\
\hline & 2.0549426 & -0.5351984 & 0.2017425 \\
\hline F & -0.9987301 & -7.5337032 & 4.8285089 \\
\hline$N$ & -0.5022960 & -1.4525795 & 0.2063520 \\
\hline$C$ & -0.3167437 & -5.4406892 & 3.3655974 \\
\hline$f$ & -2.4236298 & -7.9526004 & 3.8585852 \\
\hline F & 1.1391350 & 0.3078014 & 1.4607746 \\
\hline$c$ & 1.3023608 & 0.2506699 & 0.3729907 \\
\hline & 2.5094642 & -5.0856659 & 2.4426270 \\
\hline & 1.1406894 & -3.3836793 & 3.6642624 \\
\hline
\end{tabular}




$\begin{array}{lrrr}\text { C } & 0.9938045 & -3.5636198 & 2.5893489 \\ \text { C } & -0.0058603 & -0.1683489 & -0.2998275 \\ \text { C } & 2.2906686 & -4.1081838 & 1.9862991 \\ \text { O } & 0.4798146 & -5.5401924 & 4.3285537 \\ \text { H } & 2.7514507 & 1.8810604 & 0.3340880 \\ \text { C } & 1.8113126 & 1.5916966 & -0.1556333 \\ \text { H } & 2.1450938 & -4.2813481 & 0.9097418 \\ \text { H } & 0.1373560 & -0.2777702 & -1.3809176 \\ \text { H } & -0.7876062 & 0.5773662 & -0.1154173 \\ \text { H } & 0.7608311 & -2.5925939 & 2.1354595 \\ \text { H } & 3.6359136 & -2.9759727 & 3.2795435 \\ \text { C } & 3.4583855 & -3.1461196 & 2.2064933 \\ \text { H } & 1.9967669 & 1.5453251 & -1.2394687 \\ \text { H } & 1.0776696 & 2.3921191 & 0.0243789 \\ \text { H } & 4.3886446 & -3.5349244 & 1.7691125 \\ \text { H } & 3.2550588 & -2.1672416 & 1.7450487\end{array}$

$\mathrm{TS}(18+2 \longrightarrow 20)$

$\begin{array}{lrrr}38 & & \\ \text { Energy }=-747.6516965594 & \\ \text { H } & 4.3528915 & -2.8321341 & -2.7653058 \\ \text { H } & 3.7775567 & -0.7455660 & -1.3497127 \\ \text { O } & 1.8927393 & 0.7295398 & -2.0467790 \\ \text { H } & -1.7715939 & 1.1383759 & -1.1347011 \\ \text { C } & 3.7409374 & -3.4237739 & -2.0668913 \\ \text { C } & 3.1642388 & -1.3651123 & -0.6780074 \\ \text { C } & 1.3452807 & 0.2809900 & -0.9844585 \\ \text { H } & -0.5352784 & 1.2668902 & -2.4132518 \\ \text { H } & 4.4124402 & -3.8116775 & -1.2849817 \\ \text { H } & -0.2643153 & -2.0257010 & 3.7093079 \\ \text { C } & -0.7099290 & 1.3851087 & -1.3226865 \\ \text { H } & 3.8225015 & -1.7195744 & 0.1336200\end{array}$




$\begin{array}{lrrr}\mathrm{H} & 3.3398407 & -4.2851894 & -2.6201095 \\ \mathrm{H} & 1.3664066 & -3.6248115 & 4.8897055 \\ \mathrm{C} & 2.6254839 & -2.5712083 & -1.4600692 \\ \mathrm{~N} & 0.1061538 & 0.4794612 & -0.5421766 \\ \mathrm{~N} & 2.1368237 & -0.5092450 & -0.0990353 \\ \mathrm{H} & 2.1181550 & -2.1928394 & 2.9387788 \\ \mathrm{H} & -2.4790523 & 3.5638874 & -1.5689029 \\ \mathrm{~N} & -0.1165421 & -1.4642860 & 1.6834384 \\ \mathrm{H} & 1.9585445 & -2.1974706 & -2.2537146 \\ \mathrm{C} & -1.1102632 & -0.8634077 & 1.3051673 \\ \mathrm{C} & -0.0019297 & -2.4806211 & 2.7402344 \\ \mathrm{O} & -2.1512884 & -0.3538688 & 1.0464454 \\ \mathrm{C} & 1.4191910 & -3.0312516 & 2.7909149 \\ \mathrm{H} & -1.2474509 & 3.7174823 & -2.8388283 \\ \mathrm{C} & -1.4203666 & 3.8054325 & -1.7546182 \\ \mathrm{C} & 1.5903670 & -4.0633806 & 3.9055070 \\ \mathrm{H} & 1.5052511 & -1.0066070 & 0.5401736 \\ \mathrm{C} & -0.4988118 & 2.8669187 & -0.9728669 \\ \mathrm{H} & 2.6200457 & -4.4452639 & 3.9344547 \\ \mathrm{H} & 2.0038513 & -3.1856907 & -0.7868556 \\ \mathrm{H} & -0.7194328 & -3.2947879 & 2.5489135 \\ \mathrm{H} & -0.6617724 & 2.9929391 & 0.1109349 \\ \mathrm{H} & 0.9174660 & -4.9219561 & 3.7600791 \\ \mathrm{H} & -1.2651111 & 4.8585978 & -1.4769572 \\ \mathrm{H} & 1.6637585 & -3.4792302 & 1.8146675 \\ \mathrm{H} & 0.5562508 & 3.1199614 & -1.1670961\end{array}$

$\mathrm{TS}(20+2 \longrightarrow 21)$

51

Energy $=-1034.463201475$

H $\quad 0.8346460 \quad 1.2052693 \quad-5.4630746$

$\mathrm{H} \quad 1.7009311-0.5019351 \quad-7.2002708$ 


\begin{tabular}{|c|c|c|c|}
\hline$C$ & 1.8553177 & 1.3008673 & -5.0591394 \\
\hline C & .7038440 & -0.3772581 & -6.76273 \\
\hline I & 2.3504998 & 2.1125278 & -5.6130167 \\
\hline & 2.1263490 & -0.8087671 & -4.7089282 \\
\hline & 3.2216722 & 0.4057982 & -7.3383082 \\
\hline & L. 1404978 & 1.1641127 & -3.0090248 \\
\hline $\mathrm{N}$ & 1.7387740 & 1.7023508 & -3.6676250 \\
\hline$C$ & 2.6194861 & -0.0093834 & -5.2814212 \\
\hline 0 & 3.7862416 & 2.7343035 & -3.6371403 \\
\hline $\mathrm{H}$ & 3.2518584 & -1.3186290 & -6.9111853 \\
\hline C & 2.7799145 & 2.3041588 & -3.0328279 \\
\hline 0 & 1.2908016 & 2.8002445 & 0.2015293 \\
\hline 0 & 1.9539801 & -0.9321092 & 0.1981437 \\
\hline $\mathrm{H}$ & 4.2182048 & 3.8638232 & -1.7292238 \\
\hline $\mathrm{N}$ & 2.6395548 & 2.5117227 & -1.6506252 \\
\hline C & 1.5213383 & 2.1136570 & -0.8286877 \\
\hline C & 3.7097319 & 3.2509730 & -0.9760412 \\
\hline $\mathrm{H}$ & 3.2407275 & 3.9117618 & -0.2362611 \\
\hline $\mathrm{H}$ & 3.6274834 & 0.0986134 & -4.8539187 \\
\hline C & 2.2021128 & -0.6583643 & 049 \\
\hline $\mathrm{H}$ & 3.6244602 & -2.1588589 & -3.3043600 \\
\hline $\mathrm{N}$ & 0.8391272 & 1.0400787 & -1.2455961 \\
\hline $\mathrm{H}$ & 3.8261989 & -2.5206081 & -1.5748882 \\
\hline $\mathrm{H}$ & -0.1051923 & 0.4012477 & 0.5397 \\
\hline C & 3.8162032 & -1.7037088 & -2.3191032 \\
\hline C & 4.7127966 & 2.3210718 & -0.2909454 \\
\hline $\mathrm{H}$ & 6.3787952 & 3.7293162 & -0.2471883 \\
\hline $\mathrm{N}$ & 2.7310031 & -0.7473641 & -2.0631542 \\
\hline C & -0.3611801 & 0.7400686 & -0.4829490 \\
\hline $\mathrm{H}$ & 5.1556496 & 1.6613683 & -1.0515845 \\
\hline C & 5.8057448 & 3.0949211 & 0.4465599 \\
\hline $\mathrm{H}$ & 6.1112672 & -2.3262449 & -3.8140337 \\
\hline & 5.3753220 & 3.7513263 & 1.218 \\
\hline
\end{tabular}




$\begin{array}{lrrr}\mathrm{H} & 6.3802404 & -2.8177100 & -2.1275183 \\ \mathrm{H} & -0.9730261 & 1.6528027 & -0.3539090 \\ \mathrm{H} & 4.1687198 & 1.6687128 & 0.4114397 \\ \mathrm{C} & 6.2964901 & -1.9480107 & -2.7970712 \\ \mathrm{H} & -0.5516370 & -1.2302374 & -1.3249120 \\ \mathrm{C} & 5.1770790 & -1.0078602 & -2.3482356 \\ \mathrm{C} & -1.1855469 & -0.3402089 & -1.1803757 \\ \mathrm{H} & -2.1902481 & -1.0938505 & 0.6032368 \\ \mathrm{H} & 6.5142694 & 2.4155297 & 0.9414146 \\ \mathrm{H} & 5.1075368 & -0.1389435 & -3.0214136 \\ \mathrm{H} & 5.3940708 & -0.6089355 & -1.3451011 \\ \mathrm{C} & -2.4451539 & -0.7150057 & -0.3987188 \\ \mathrm{H} & 7.2682903 & -1.4346338 & -2.8016294 \\ \mathrm{H} & -1.4551429 & 0.0129306 & -2.1895945 \\ \mathrm{H} & -3.1041725 & 0.1569981 & -0.2651633 \\ \mathrm{H} & -3.0236680 & -1.4952215 & -0.9139505\end{array}$

$\mathrm{TS}(21+2 \longrightarrow 22)$

\begin{tabular}{lrrr}
\multicolumn{4}{l}{64} \\
\multicolumn{4}{l}{ Energy $=-1321.257882244$} \\
H & -2.8233425 & 2.4030339 & -1.7648631 \\
H & -0.7317768 & 2.2411928 & -0.3404882 \\
H & -2.9533890 & 3.4258065 & -0.3176209 \\
C & -2.8737240 & 2.3864014 & -0.6653812 \\
C & -1.6484107 & 1.6936279 & -0.0678313 \\
H & -1.4355439 & 0.2108601 & -1.6403042 \\
O & 2.2755213 & -0.8297882 & 0.0814279 \\
H & 0.5742768 & -0.2750679 & -0.3725842 \\
H & 4.4139766 & -1.7982879 & 0.4487921 \\
H & 5.9827943 & -2.5820334 & -1.4834662 \\
C & -1.5193827 & 0.2417666 & -0.5435776 \\
H & -3.8006242 & 1.8650313 & -0.3815345
\end{tabular}




\begin{tabular}{|c|c|c|c|}
\hline $\mathrm{I}$ & .7145502 & -3.3508316 & Q236 \\
\hline 4 & -1.7071859 & 1.6966594 & 1.0318137 \\
\hline $\mathrm{N}$ & -0.3610974 & 51345 & 1 \\
\hline 0 & 1.0705105 & -4.4627000 & 1.2588877 \\
\hline$C$ & 4.0756966 & -2.8376229 & 0.5429925 \\
\hline C & 1.9618265 & -1.7146104 & 0.9119380 \\
\hline C & 5.5726334 & -3.5950792 & -1.3523476 \\
\hline $\mathrm{H}$ & 6.2368627 & -4.1367305 & -0.6615789 \\
\hline C & 2.2019443 & -4.0858042 & 1.6630690 \\
\hline $\mathrm{N}$ & 2.7231394 & -2.8215490 & 1.1024787 \\
\hline $\mathrm{H}$ & -2.4192965 & -0.3233758 & -0.2626232 \\
\hline C & -0.3968530 & -1.0202376 & 1.2353630 \\
\hline C & 4.1432780 & -3.5441061 & -0.8116191 \\
\hline 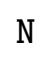 & 0.8055879 & -1.5852559 & 1.7136242 \\
\hline $\mathrm{H}$ & 2.0981723 & -6.5396776 & 2.3188350 \\
\hline $\mathrm{H}$ & 5.6147172 & -4.1027271 & -2.3261837 \\
\hline $\mathrm{H}$ & 3.5188399 & -6.4674906 & 3.3947369 \\
\hline $\mathrm{H}$ & 3.4832893 & -3.0200935 & -1.5205364 \\
\hline 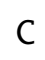 & 2.6191014 & -5.9162253 & 3.0707230 \\
\hline 0 & -1.4315579 & -1.0990616 & H. \\
\hline$N$ & 3.0468464 & -4.6556350 & 2.4931861 \\
\hline $\mathrm{H}$ & 3.7458832 & -4.5659980 & -0.6989370 \\
\hline$C$ & 0.7922830 & -2.0417839 & 3.1130471 \\
\hline $\mathrm{H}$ & 1.6868239 & -2.6482862 & 3.2794668 \\
\hline$c$ & 4.4483137 & -3.5439361 & 3.9450369 \\
\hline $\mathrm{H}$ & -0.0912839 & -2.6718116 & 3.2763900 \\
\hline $\mathrm{H}$ & 2.1640366 & -7.6283969 & 5.2555336 \\
\hline 0 & 5.0958146 & -4.4928967 & 4.2861005 \\
\hline $\mathrm{H}$ & 0.7357508 & -7.6908962 & 4.2013640 \\
\hline$c$ & 1.2826938 & -7.0599395 & 4.9191785 \\
\hline C & 1.6945642 & -5.7320891 & 4.2826703 \\
\hline $\mathrm{N}$ & 3.9816979 & -2.4146408 & 3.8314181 \\
\hline C & 0.8265966 & -0.8793330 & 4.10557 \\
\hline
\end{tabular}




$\begin{array}{lrrr}\mathrm{H} & 0.8051687 & -5.1707104 & 3.9569858 \\ \mathrm{H} & 2.2102381 & -5.0992024 & 5.0230852 \\ \mathrm{H} & 1.7173349 & -0.2663518 & 3.8949498 \\ \mathrm{H} & 5.5313025 & -1.3741399 & 4.8641845 \\ \mathrm{H} & -0.0522853 & -0.2341079 & 3.9612725 \\ \mathrm{H} & 0.6317365 & -6.9060572 & 5.7921887 \\ \mathrm{C} & 4.5675146 & -1.1821646 & 4.3621593 \\ \mathrm{C} & 0.8792464 & -1.3994620 & 5.5424816 \\ \mathrm{H} & 1.7602808 & -2.0421114 & 5.6896038 \\ \mathrm{H} & 5.4579634 & -0.5496944 & 2.5069044 \\ \mathrm{H} & 3.8766257 & -0.7802885 & 5.1217017 \\ \mathrm{H} & -0.0131797 & -1.9986563 & 5.7792918 \\ \mathrm{C} & 4.7477712 & -0.1516098 & 3.2481996 \\ \mathrm{H} & 6.2058891 & 1.0932434 & 4.2936543 \\ \mathrm{H} & 0.9323981 & -0.5744687 & 6.2665297 \\ \mathrm{H} & 3.7863597 & -0.0313373 & 2.7244760 \\ \mathrm{C} & 5.2355453 & 1.1944382 & 3.7842060 \\ \mathrm{H} & 4.5205634 & 1.6151916 & 4.5076771 \\ \mathrm{H} & 5.3584568 & 1.9232579 & 2.9710808\end{array}$

\section{$\mathrm{TS}(22 \longrightarrow 23)$}

\begin{tabular}{|c|c|c|c|}
\hline \multicolumn{4}{|c|}{ Energy $=-1321.275337671$} \\
\hline $\mathrm{H}$ & -2.6716402 & -7.2494995 & -2.8319095 \\
\hline 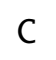 & -1.9739312 & -7.0547081 & -2.0046008 \\
\hline $\mathrm{H}$ & -0.9772167 & -7.4020190 & -2.3182804 \\
\hline $\mathrm{H}$ & -2.2862741 & -7.6747278 & -1.1498187 \\
\hline $\mathrm{H}$ & -4.6241332 & -1.2616441 & 4.8360991 \\
\hline $\mathrm{H}$ & -2.9515068 & -5.2425650 & -1.3143013 \\
\hline $\mathrm{C}$ & -1.9465987 & -5.5729494 & -1.6251204 \\
\hline$H$ & -1.6745962 & -4.9619502 & -2.5023848 \\
\hline & -3.7171501 & -2.2815321 & 2.6469749 \\
\hline
\end{tabular}




\begin{tabular}{|c|c|c|c|}
\hline & -3.7727768 & -0.5640235 & 1203 \\
\hline H & -4.1003248 & 0.3589950 & 4.3281301 \\
\hline & -2.1433586 & -9.0870663 & 6.5020835 \\
\hline & -3.1910319 & -0.6694791 & 2.1062525 \\
\hline & -2.8937628 & -1.5567234 & .6752057 \\
\hline & -3.5360985 & 3049 & 5.8734549 \\
\hline & -1.2048779 & -6.9320461 & 5.5971540 \\
\hline & -2.7500060 & -7.3097341 & 4.8215261 \\
\hline & -0.9626907 & -5.2730287 & 384285 \\
\hline 0 & -2.6 & 815361 & 2597 \\
\hline C & -1.7053082 & -7.6448916 & 4.9236551 \\
\hline c & -2.5652291 & -1.1769247 & 4.1196236 \\
\hline C & -1.6595341 & -9.0539468 & 5.5159997 \\
\hline & 0.0573124 & -5.5637520 & -0.8027813 \\
\hline & -1.2291416 & -5 & 053 \\
\hline H & -2.2284062 & -2.0759520 & 4.6616147 \\
\hline H & -1.9332498 & -5.8845268 & 2.5622523 \\
\hline $\mathrm{N}$ & -1.7498022 & -2.1704148 & 1.9689776 \\
\hline C & -1.5 & -3. & 23 \\
\hline$N$ & -1.0251624 & -3.8832893 & -0.0772499 \\
\hline H & -0.6211813 & -9.3974728 & 5.6392599 \\
\hline $\mathrm{H}$ & -2.1752445 & -9.7755895 & 4.8642227 \\
\hline $\mathrm{H}$ & -1.7219804 & -0.4695752 & 4.117 \\
\hline $\mathrm{N}$ & -1.0569987 & -0.4 & 91 \\
\hline C & -1.0261092 & -7.5782609 & 3.5508795 \\
\hline C & -0.9656341 & -1.3098785 & 1.1948677 \\
\hline 0 & -1.1228185 & -0.0802091 & 1.3332578 \\
\hline C & 0.0659774 & -3.1717685 & -0.2201915 \\
\hline 0 & 1.1839147 & -3.4591057 & -0.7369022 \\
\hline N & -0.3837828 & -3.9696387 & 2.8284787 \\
\hline $\mathrm{H}$ & 2.6299282 & -1.1039285 & 0.6193 \\
\hline $\mathrm{H}$ & 0.0231849 & -7.8936690 & 3.636993 \\
\hline & -0.0513702 & -1.7986223 & 0.2893890 \\
\hline
\end{tabular}




$\begin{array}{lrrr}\mathrm{C} & -0.1823098 & -5.2953010 & 3.3392020 \\ \mathrm{H} & -1.5236426 & -8.2645065 & 2.8497213 \\ \mathrm{H} & 1.5790803 & 0.0887585 & 1.3970671 \\ \mathrm{C} & 1.9453235 & -0.2667993 & 0.4220692 \\ \mathrm{H} & 2.3366320 & -4.2440967 & 2.7424579 \\ \mathrm{H} & 0.7594365 & -3.1310922 & 4.3575122 \\ \mathrm{C} & 0.6556284 & -3.0122376 & 3.2693830 \\ \mathrm{C} & 0.7627838 & -0.7804650 & -0.3970250 \\ \mathrm{C} & 2.0223774 & -3.2000425 & 2.6125754 \\ \mathrm{O} & 0.7903935 & -5.5185244 & 4.0752652 \\ \mathrm{H} & 3.5397258 & 1.2188118 & 0.2880528 \\ \mathrm{C} & 2.6843392 & 0.8594573 & -0.3013009 \\ \mathrm{H} & 1.9439253 & -3.0147033 & 1.5330866 \\ \mathrm{H} & 1.1296272 & -1.2521889 & -1.3152536 \\ \mathrm{H} & 0.1062889 & 0.0590100 & -0.6649118 \\ \mathrm{H} & 0.2795866 & -2.0022856 & 3.0888755 \\ \mathrm{H} & 3.1714463 & -2.4817393 & 4.3234314 \\ \mathrm{C} & 3.0539075 & -2.2682087 & 3.2497738 \\ \mathrm{H} & 3.0678854 & 0.5208604 & -1.2762028 \\ \mathrm{H} & 2.0184870 & 1.7166708 & -0.4847651 \\ \mathrm{H} & 4.0383156 & -2.3831039 & 2.7748630 \\ \mathrm{H} & 2.7569920 & -1.2135849 & 3.1498571\end{array}$

$$
\mathrm{TS}(23 \longrightarrow 6+18)
$$

\begin{tabular}{lrrr}
64 \\
\multicolumn{4}{l}{ Energy $=-1321.298220521$} \\
H & -1.3313267 & -7.0449405 & -3.2253510 \\
C & -0.6844497 & -6.7736322 & -2.3795103 \\
H & 0.3484990 & -6.7069150 & -2.7542257 \\
H & -0.7221429 & -7.5966632 & -1.6495867 \\
H & -3.4788752 & -3.2751815 & 5.5793747 \\
H & -2.1619722 & -5.5321168 & -1.3798222
\end{tabular}




\begin{tabular}{|c|c|c|c|}
\hline$C$ & -1.1269840 & -5.4549190 & -1 . \\
\hline $\mathrm{H}$ & -1.1035508 & -4.6470250 & -2.4920535 \\
\hline UT & -3.1475159 & -3.6 & 3.0021382 \\
\hline C & -2.8693315 & -2.3628423 & 5.4964135 \\
\hline H & -3.5573388 & -1.5120673 & 5.3752310 \\
\hline $\mathrm{H}$ & -4.0063123 & -6.3033601 & 6.8579732 \\
\hline$\Pi$ & -3.3954195 & -1.8863508 & 2.8661405 \\
\hline C & -2.6404419 & -2.6724891 & 3.0052921 \\
\hline $\mathrm{H}$ & -2.3345667 & -2.2306537 & 6.4472834 \\
\hline H & -2.1124124 & -5.3668266 & 5.4814861 \\
\hline n & -3.4750945 & -5.8056886 & 4.4449822 \\
\hline C & -0.2258971 & -5.0717078 & -0.5709486 \\
\hline 0 & -2.3175528 & -4.8501010 & 1.2547868 \\
\hline C & -2.6985018 & -6.2209969 & 5.1083111 \\
\hline 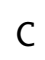 & -1.8937352 & -2.45 & 12 \\
\hline C & -3.3480962 & -6.9641612 & 6.27 \\
\hline $\mathrm{H}$ & 0.8123626 & -4.9584239 & -0.90 \\
\hline $\mathrm{H}$ & -0.2535408 & -5.8382460 & 0.2136542 \\
\hline $\mathrm{H}$ & -1.1878365 & -3.2846559 & 4.4731996 \\
\hline $\mathrm{H}$ & -1 . & -6.0970 & 97 \\
\hline $\mathrm{N}$ & -1.7675411 & -2.6698317 & 1.8245242 \\
\hline$c$ & -1.4438812 & -3.9493849 & 1.2438438 \\
\hline $\mathrm{N}$ & -0.6195789 & -3.8156776 & 0.0726299 \\
\hline $\mathrm{H}$ & -2.5856236 & -7.3654821 & 6.961 \\
\hline $\mathrm{H}$ & -3.9545780 & -7.8114649 & 5.920 \\
\hline 1 & -1.3006743 & -1.5324763 & 4.251 \\
\hline $\mathrm{N}$ & -1.1236041 & -6.4384273 & 3.1629226 \\
\hline C & -1.7664443 & -7.1168608 & 4.2820322 \\
\hline C & -1.3327806 & -1.4571897 & 1.36237 \\
\hline 0 & -1.6648648 & -0.3862669 & 1.8882510 \\
\hline C & -0.0994268 & -2.6485362 & -0.4103229 \\
\hline 0 & 0.6655391 & -2.6070219 & -1.3835868 \\
\hline $\mathrm{N}$ & 0.0084081 & -4.5312347 & 2.4983 \\
\hline
\end{tabular}




$\begin{array}{rrrr}\mathrm{H} & 2.0834620 & -0.6050429 & 0.2258349 \\ \mathrm{H} & -0.9806540 & -7.5244110 & 4.9348443 \\ \mathrm{~N} & -0.5040467 & -1.4780343 & 0.2369216 \\ \mathrm{C} & -0.1306229 & -5.4897562 & 3.4354826 \\ \mathrm{H} & -2.3342619 & -7.9718490 & 3.8802853 \\ \mathrm{H} & 1.1881864 & 0.2201830 & 1.5116123 \\ \mathrm{C} & 1.3429264 & 0.1854450 & 0.4220057 \\ \mathrm{H} & 2.6998737 & -4.9506784 & 2.3281377 \\ \mathrm{H} & 1.2953251 & -3.4401793 & 3.7561079 \\ \mathrm{C} & 1.0799735 & -3.5763661 & 2.6813689 \\ \mathrm{C} & 0.0257938 & -0.1984930 & -0.2522250 \\ \mathrm{C} & 2.3803718 & -3.9613743 & 1.9631881 \\ \mathrm{O} & 0.5843122 & -5.6080801 & 4.4740456 \\ \mathrm{H} & 2.8125656 & 1.7959055 & 0.4051963 \\ \mathrm{C} & 1.8632851 & 1.5318789 & -0.0809020 \\ \mathrm{H} & 2.1757716 & -4.0731327 & 0.8866180 \\ \mathrm{H} & 0.1631998 & -0.3002424 & -1.3348234 \\ \mathrm{H} & -0.7407016 & 0.5611137 & -0.0599638 \\ \mathrm{H} & 0.7498853 & -2.5936645 & 2.3054492 \\ \mathrm{H} & 3.7291512 & -2.8307354 & 3.2523511 \\ \mathrm{C} & 3.4914093 & -2.9341032 & 2.1822350 \\ \mathrm{H} & 2.0363671 & 1.5089133 & -1.1674410 \\ \mathrm{H} & 1.1427721 & 2.3373208 & 0.1269088 \\ \mathrm{H} & 4.4159891 & -3.2160563 & 1.6582024 \\ \mathrm{H} & 3.1881806 & -1.9417829 & 1.8146409\end{array}$

$$
\mathrm{TS}(21 \longrightarrow 24+18)
$$

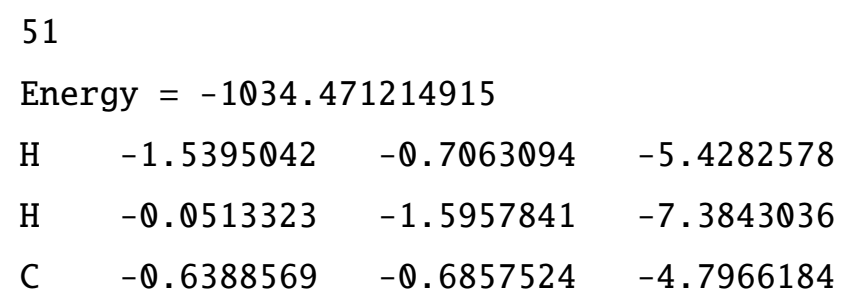




\begin{tabular}{|c|c|c|c|}
\hline & .8021896 & -1.5812255 & -6 \\
\hline & 33402 & 0. 3014940 & 5278 \\
\hline & -0.1451759 & -2.7585063 & -5.1308300 \\
\hline & 1.3167267 & -0.6160461 & -6.8132518 \\
\hline & -1.9425505 & -1.2898640 & 3.1647416 \\
\hline & -1.0654420 & -0.8260452 & -3.4161437 \\
\hline C & 0.3402397 & -1.7770379 & 1535 \\
\hline & 0.7094244 & 0. 3354042 & -2.5582748 \\
\hline & 1.4979088 & -2.3743518 & 968599 \\
\hline & -0.3211417 & -0.3498119 & -2.3836681 \\
\hline c & -2.8976387 & -1.5113393 & -1.5672318 \\
\hline c & -0.9166767 & -4.1739721 & 1.1800118 \\
\hline & 0.4167719 & 0.8048075 & -0.2462131 \\
\hline & -0.7730381 & -0.6582872 & 967186 \\
\hline C & -1.8460816 & -1 . & 256 \\
\hline c & 0.0678263 & -0.2033099 & 0.0158352 \\
\hline & -0.5732952 & -0.1245829 & 0.9011915 \\
\hline $\mathrm{H}$ & 1.2043004 & -1.7706513 & -4.5624750 \\
\hline C & -1.2156353 & -3.3498167 & 0.2870681 \\
\hline & 0.7379791 & -4.5277395 & -0.9002556 \\
\hline & -1.8901016 & -2.1266669 & 0.4833817 \\
\hline & 0.8659294 & -3.1262246 & -1.9822025 \\
\hline & -3.9868651 & -2.2032619 & 0.6951260 \\
\hline C & 0.18 & -3 & -1 \\
\hline C & 1.2793118 & -1.0854503 & 0.3293775 \\
\hline $\mathrm{H}$ & 2.5232476 & 0.5231725 & 1.1241769 \\
\hline $\mathrm{N}$ & -0.9844474 & -3.3284422 & -1.0405143 \\
\hline C & -3.0853720 & -1.9943951 & 1.3018175 \\
\hline $\mathrm{H}$ & 1.8668904 & -1.2184041 & -0.5912051 \\
\hline C & 2.1452510 & -0.4656502 & 1.4269283 \\
\hline $\mathrm{H}$ & 1.7030621 & -5.9259745 & -2.9200744 \\
\hline $\mathrm{H}$ & 1.5704334 & -0.3314748 & 2.3564420 \\
\hline & 1.6799927 & -4.4854256 & -3.958877 \\
\hline
\end{tabular}




$\begin{array}{lrrr}\text { H } & -3.0172668 & -2.7749131 & 2.0748351 \\ \text { H } & 0.9368462 & -2.0818240 & 0.6426648 \\ \text { C } & 1.0684122 & -5.3216422 & -3.5866501 \\ \text { H } & -3.2072753 & 0.1532710 & 1.1626525 \\ \text { C } & -0.1697485 & -4.8066680 & -2.8530943 \\ \text { C } & -3.2104007 & -0.6166297 & 1.9516844 \\ \text { H } & -5.3798081 & -0.6513552 & 2.1809738 \\ \text { H } & 3.0123190 & -1.0999818 & 1.6591760 \\ \text { H } & -0.7918111 & -5.6505619 & -2.5120219 \\ \text { H } & -0.7959416 & -4.2127445 & -3.5381714 \\ \mathrm{C} & -4.4801788 & -0.4902347 & 2.7944045 \\ \text { H } & 0.7967559 & -5.9471902 & -4.4488163 \\ \text { H } & -2.3198826 & -0.4339932 & 2.5741933 \\ \text { H } & -4.4919447 & -1.2345531 & 3.6053452 \\ \text { H } & -4.5597513 & 0.5059975 & 3.2514466\end{array}$




\subsection{Cartesian Coordinates Catalyst Decomposition}

All coordinates are given in Ångström in xyz format. The energy given is the BP86-D3cosmo/def2-TZVP energy of the optimized structure. The energy is given in Hartree. The first lines of the cartesian coordinate inputs refer to the number of atoms.

\subsubsection{General Compounds}

\begin{tabular}{|c|c|c|c|}
\hline \multicolumn{4}{|c|}{$\mathrm{NPr}_{4}{ }^{+}$} \\
\hline \multicolumn{4}{|c|}{41} \\
\hline \multicolumn{4}{|c|}{ Energy $=-529.0041690028$} \\
\hline N & 0.7711458 & 1.0422101 & 3.8506431 \\
\hline$c$ & -0.0144810 & 1.9135131 & 4.8209079 \\
\hline C & 1.2089095 & 1.8396040 & 2.6297081 \\
\hline$C$ & 2.0465399 & 0.5155458 & 4.4954681 \\
\hline 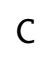 & -0.1556187 & -0.1000015 & 3.4569595 \\
\hline C & 0.4187820 & -1.1138019 & 2.4809777 \\
\hline $\mathrm{H}$ & -0.4480392 & -0.5854660 & 4.3956953 \\
\hline $\mathrm{H}$ & -1.0541657 & 0.3720163 & 3.0441180 \\
\hline $\mathrm{H}$ & 1.7929593 & 1.1469999 & 2.0128541 \\
\hline C & 0.0918396 & 2.4755441 & 1.8176637 \\
\hline $\mathrm{H}$ & 1.9020088 & 2.6025529 & 3.0030031 \\
\hline $\mathrm{H}$ & 2.5660956 & -0.0503733 & 3.7133017 \\
\hline $\mathrm{H}$ & 2.6584270 & 1.3985491 & 4.7147523 \\
\hline 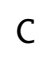 & 1.8586356 & -0.3339559 & 5.7416507 \\
\hline त & -0.3102925 & 1.2534045 & 5.6446544 \\
\hline C & 0.7102766 & 3.1425168 & 5.3462558 \\
\hline H & -0.9313025 & 2.1998743 & 4.2917344 \\
\hline C & -0.6277004 & -2.1964844 & 2.1955582 \\
\hline 1 & 0.7057317 & -0.6272042 & 1.5377115 \\
\hline 1 & 1.3238447 & -1.5817546 & 2.8930756 \\
\hline C & 3.2281263 & -0.7694951 & 6.2734156 \\
\hline & 1.3281456 & 0.2301229 & 6.5218308 \\
\hline
\end{tabular}




$\begin{array}{rrrr}\mathrm{H} & 1.2547347 & -1.2247037 & 5.5176820 \\ \mathrm{C} & -0.2064157 & 3.9017889 & 6.3111323 \\ \mathrm{H} & 1.6331343 & 2.8564360 & 5.8706600 \\ \mathrm{H} & 0.9985041 & 3.8070714 & 4.5195320 \\ \mathrm{C} & 0.6930009 & 3.2312935 & 0.6277901 \\ \mathrm{H} & -0.6053342 & 1.7101155 & 1.4482883 \\ \mathrm{H} & -0.4901468 & 3.1730098 & 2.4362165 \\ \mathrm{H} & -0.4860508 & 3.2730440 & 7.1686823 \\ \mathrm{H} & -1.1302239 & 4.2241286 & 5.8094997 \\ \mathrm{H} & 0.3011009 & 4.7957573 & 6.6956253 \\ \mathrm{H} & 3.7736740 & -1.3614652 & 5.5246016 \\ \mathrm{H} & 3.1095584 & -1.3857066 & 7.1738553 \\ \mathrm{H} & 3.8466844 & 0.1010257 & 6.5355164 \\ \mathrm{H} & -0.2291616 & -2.9358525 & 1.4889824 \\ \mathrm{H} & -1.5374295 & -1.7619225 & 1.7572422 \\ \mathrm{H} & -0.9122154 & -2.7243997 & 3.1170888 \\ \mathrm{H} & 1.2574046 & 2.5528366 & -0.0279122 \\ \mathrm{H} & 1.3757378 & 4.0240807 & 0.9656413 \\ \mathrm{H} & -0.1015044 & 3.6980852 & 0.0314861\end{array}$

\begin{tabular}{|c|c|c|c|}
\hline \multicolumn{4}{|c|}{$\mathrm{NF}$} \\
\hline \multicolumn{4}{|c|}{31} \\
\hline \multicolumn{4}{|c|}{ Energy $=-410.5491046693$} \\
\hline N & 0.6554896 & 0.9097362 & 4.0567793 \\
\hline C & -0.0732779 & 1.7950062 & 4.9735204 \\
\hline 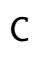 & 1.9587621 & 0.4671818 & 4.5764784 \\
\hline$C$ & -0.2123174 & -0.1938612 & 3.6268345 \\
\hline 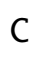 & 0.3923139 & -1.0816398 & 2.5411391 \\
\hline $\mathrm{H}$ & -0.5287207 & -0.8328137 & 4.4821554 \\
\hline & -1.1347756 & 0.2651798 & 3.2343293 \\
\hline & 2.4713351 & -0.0829419 & 3.7728531 \\
\hline 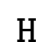 & 2.5693611 & 1.3646282 & 4.7598218 \\
\hline
\end{tabular}




$\begin{array}{rrrr}\mathrm{C} & 1.9508619 & -0.3982525 & 5.8465290 \\ \mathrm{H} & -0.3736161 & 1.2705787 & 5.9088020 \\ \mathrm{C} & 0.6763031 & 3.0748221 & 5.3392972 \\ \mathrm{H} & -1.0131070 & 2.0703240 & 4.4662729 \\ \mathrm{C} & -0.6358886 & -2.0664731 & 1.9805910 \\ \mathrm{H} & 0.7837127 & -0.4400755 & 1.7345896 \\ \mathrm{H} & 1.2497876 & -1.6449609 & 2.9413853 \\ \mathrm{C} & 3.3718392 & -0.7770038 & 6.2677362 \\ \mathrm{H} & 1.4537171 & 0.1441159 & 6.6665057 \\ \mathrm{H} & 1.3598088 & -1.3121697 & 5.6742821 \\ \mathrm{C} & -0.2150529 & 4.0473943 & 6.1151337 \\ \mathrm{H} & 1.5641870 & 2.8377429 & 5.9457431 \\ \mathrm{H} & 1.0423681 & 3.5489511 & 4.4139718 \\ \mathrm{H} & -0.5862504 & 3.5887533 & 7.0443174 \\ \mathrm{H} & -1.0898551 & 4.3467443 & 5.5179405 \\ \mathrm{H} & 0.3340228 & 4.9592661 & 6.3889714 \\ \mathrm{H} & 3.8813612 & -1.3433794 & 5.4728682 \\ \mathrm{H} & 3.3737053 & -1.3968708 & 7.1754903 \\ \mathrm{H} & 3.9749717 & 0.1210031 & 6.4735026 \\ \mathrm{H} & -0.1893476 & -2.7160860 & 1.2147536 \\ \mathrm{H} & -1.4841009 & -1.5366906 & 1.5211020 \\ \mathrm{H} & -1.0375579 & -2.7137792 & 2.7751231\end{array}$

\section{Propene}

9

Energy $=-117.9617418642$

$\begin{array}{lrrr}\text { C } & -0.0685981 & -0.1702666 & -0.0343711 \\ \text { C } & 0.0450959 & 0.1725148 & 1.2540691 \\ \text { C } & 1.2883432 & 0.0340621 & 2.0783748 \\ \text { H } & 1.1105938 & -0.6147355 & 2.9511748 \\ \text { H } & -0.8284203 & 0.5897539 & 1.7681884 \\ \text { H } & -1.0058741 & -0.0434849 & -0.5792074\end{array}$




$\begin{array}{lrrr}\mathrm{H} & 0.7755576 & -0.5902533 & -0.5884462 \\ \mathrm{H} & 2.1175814 & -0.3881247 & 1.4936987 \\ \mathrm{H} & 1.6049318 & 1.0105343 & 2.4790179\end{array}$

\subsubsection{Carboxylate Catalysis}

\section{Compound $1+\mathrm{NPr}_{4}^{+}$}

$\begin{array}{lrrl}\text { 48 } & & & \\ \text { Energy }=-757.7645187739 & \\ \mathrm{H} & 0.0037782 & 3.8601049 & 0.0764097 \\ \mathrm{H} & 1.3941485 & 4.1876421 & 1.1351309 \\ \mathrm{C} & 0.7631446 & 3.3910501 & 0.7152886 \\ \mathrm{H} & 1.3960549 & 2.7542954 & 0.0806628 \\ \mathrm{H} & -0.5500191 & 3.2340245 & 2.4230609 \\ \mathrm{C} & 0.0950075 & 2.5739227 & 1.8260770 \\ \mathrm{H} & -1.1912511 & 4.2964235 & 5.8388255 \\ \mathrm{H} & 1.0386484 & 3.7715668 & 4.7807286 \\ \mathrm{H} & 0.1555456 & 4.6795682 & 6.9345101 \\ \mathrm{H} & -0.5514636 & 1.8075176 & 1.3756256 \\ \mathrm{H} & 1.8235914 & 2.6938087 & 3.1260867 \\ \mathrm{C} & -0.3672263 & 3.8613334 & 6.4225898 \\ \mathrm{C} & 0.5993918 & 3.0864082 & 5.5204120 \\ \mathrm{C} & 1.1632991 & 1.9312504 & 2.6962292 \\ \mathrm{H} & -0.9899046 & 2.3508778 & 4.2532751 \\ \mathrm{H} & 1.7917099 & 1.2571942 & 2.1018704 \\ \mathrm{H} & -0.8038163 & 3.2042951 & 7.1886115 \\ \mathrm{H} & 1.4260283 & 2.6930940 & 6.1292524 \\ \mathrm{C} & -0.1526059 & 1.9544667 & 4.8404938 \\ \mathrm{O} & -3.3638288 & -1.8251570 & 5.6785224 \\ \mathrm{H} & -1.1366112 & 0.5038914 & 2.9595218 \\ \mathrm{~N} & 0.6594761 & 1.1052845 & 3.8704153 \\ \mathrm{H} & -3.2833509 & 0.6902660 & 4.2568219 \\ \mathrm{H} & 2.5323082 & 1.3900018 & 4.7864953\end{array}$




$\begin{array}{rrrr}\mathrm{H} & -0.5779020 & 1.2487046 & 5.5709696 \\ \mathrm{H} & 0.6817017 & -0.5159363 & 1.5249889 \\ \mathrm{C} & -2.8048876 & -0.6995328 & 5.8392673 \\ \mathrm{C} & -0.2739652 & -0.0038207 & 3.4080128 \\ \mathrm{C} & -3.5491910 & 0.5389546 & 5.3149495 \\ \mathrm{H} & -3.2619655 & 1.4447624 & 5.8640722 \\ \mathrm{C} & 1.8940636 & 0.5308771 & 4.5470127 \\ \mathrm{H} & -1.6182214 & -1.5393819 & 1.5616256 \\ \mathrm{H} & 2.4142984 & -0.0522974 & 3.7778369 \\ \mathrm{C} & 0.3129215 & -1.0119365 & 2.4344525 \\ \mathrm{H} & -0.6252586 & -0.4913962 & 4.3262256 \\ \mathrm{O} & -1.6633231 & -0.5104256 & 6.3685555 \\ \mathrm{H} & -4.6371459 & 0.4002698 & 5.3628750 \\ \mathrm{C} & -0.7685067 & -2.0304591 & 2.0573534 \\ \mathrm{H} & 1.1354684 & 0.2834927 & 6.5598308 \\ \mathrm{H} & 1.1660644 & -1.5373584 & 2.8864403 \\ \mathrm{C} & 1.6369907 & -0.3123645 & 5.7851888 \\ \mathrm{H} & -0.3639756 & -2.7851529 & 1.3706559 \\ \mathrm{H} & 3.6482819 & -0.0280195 & 6.5906786 \\ \mathrm{H} & -1.1505654 & -2.5487859 & 2.9487605 \\ \mathrm{H} & 0.9631654 & -1.1485425 & 5.5550728 \\ \mathrm{C} & 2.9670653 & -0.8487584 & 6.3225723 \\ \mathrm{H} & 3.4724869 & -1.4782863 & 5.5756904 \\ \mathrm{H} & 2.7996450 & -1.4571076 & 7.2208950\end{array}$

\section{Compound $1+\mathrm{Pr}^{+}$}

17

Energy $=-347.2018730017$

$\begin{array}{llll}\mathrm{H} & -3.6009144 & -1.7221313 & 1.8786323 \\ \mathrm{C} & -3.2361380 & -0.7018902 & 1.6979362 \\ \mathrm{O} & -0.9355160 & -1.2091784 & 2.2458233 \\ \mathrm{C} & -1.7523875 & -0.7532115 & 1.4564629\end{array}$




$\begin{array}{rrrr}\text { H } & -3.7768181 & -0.2595236 & 0.8554401 \\ \mathrm{H} & -3.4262098 & -0.1171516 & 2.6082973 \\ \mathrm{O} & -1.4133175 & -0.2336117 & 0.2572198 \\ \mathrm{H} & 0.5470542 & 0.3297736 & 0.7048575 \\ \mathrm{C} & 0.0103980 & -0.2369637 & -0.0698711 \\ \mathrm{H} & 0.3698426 & -1.2761349 & -0.0494804 \\ \mathrm{C} & 0.1724209 & 0.3891787 & -1.4415352 \\ \mathrm{H} & 2.2471331 & 1.0115599 & -1.1556670 \\ \mathrm{H} & -0.2401484 & 1.4098145 & -1.4228598 \\ \mathrm{C} & 1.6437999 & 0.4237440 & -1.8632954 \\ \mathrm{H} & 2.0690694 & -0.5898895 & -1.9078813 \\ \mathrm{H} & -0.4174065 & -0.1857462 & -2.1722145 \\ \mathrm{H} & 1.7528013 & 0.8784586 & -2.8570241\end{array}$

\section{Compound $1+\mathrm{H}^{+}$}

8

Energy $=-229.2202348430$

$\begin{array}{lrrr}\text { C } & -4.5109850 & 0.2482695 & 5.9651375 \\ \text { C } & -3.1170160 & 0.3557830 & 5.4160106 \\ \text { O } & -3.0579135 & 1.0814025 & 4.2730800 \\ \text { O } & -2.1239012 & -0.1455491 & 5.9237939 \\ \text { H } & -4.5241765 & 0.6728735 & 6.9781269 \\ \text { H } & -4.7759893 & -0.8141444 & 6.0504915 \\ \text { H } & -5.2442125 & 0.7645459 & 5.3381820 \\ \text { H } & -2.1197791 & 1.1040111 & 3.9822966\end{array}$

$\mathrm{TS}\left(1+\mathrm{NPr}_{4}{ }^{+} \longrightarrow 1+\mathrm{Pr}^{+}+\mathrm{NPr}_{3}\right)$

48

Energy $=-757.7179418850$

$\begin{array}{llll}\mathrm{H} & 2.4555510 & -1.4495637 & 7.3100856\end{array}$

$\begin{array}{llll}\text { C } & 2.6808026 & -0.7877587 & 6.4629466\end{array}$

$\mathrm{H} \quad 3.4386867 \quad-1.2842551 \quad 5.8386285$ 


$\begin{array}{rrrr}\mathrm{H} & 0.9742534 & -1.4279202 & 5.3051146 \\ \mathrm{H} & -0.8244081 & -2.9274283 & 2.5650427 \\ \mathrm{C} & 1.4157639 & -0.4833910 & 5.6564251 \\ \mathrm{H} & 3.1267670 & 0.1348411 & 6.8633793 \\ \mathrm{H} & 0.6687684 & -0.0069732 & 6.3096848 \\ \mathrm{C} & -0.4329079 & -2.3506695 & 1.7136712 \\ \mathrm{H} & 0.0810515 & -3.0500730 & 1.0402489 \\ \mathrm{H} & -4.1755451 & 1.9824224 & 8.5137822 \\ \mathrm{H} & 1.3773570 & -1.6936034 & 2.7013675 \\ \mathrm{H} & -0.6062352 & -0.8289430 & 3.9897137 \\ \mathrm{O} & -1.9546691 & 0.8226747 & 7.4597068 \\ \mathrm{C} & 0.5133709 & -1.2455016 & 2.1872654 \\ \mathrm{C} & 1.7421035 & 0.4243315 & 4.4745272 \\ \mathrm{H} & -1.2921264 & -1.9286737 & 1.1710754 \\ \mathrm{C} & -4.2493975 & 1.5305754 & 7.5141211 \\ \mathrm{H} & -0.9437843 & 0.8613323 & 5.4154862 \\ \mathrm{C} & -0.2117230 & -0.2780241 & 3.1245565 \\ \mathrm{H} & 2.4879553 & -0.0711898 & 3.8251174 \\ \mathrm{C} & -2.8570147 & 1.4772657 & 6.8862101 \\ \mathrm{H} & -4.6293673 & 0.5072479 & 7.6438582 \\ \mathrm{H} & -1.4782742 & 1.9332740 & 3.9919812 \\ \mathrm{H} & 2.2217000 & 1.3441870 & 4.8412604 \\ \mathrm{H} & -0.7039397 & 3.9896843 & 6.3019665 \\ \mathrm{H} & -2.7223869 & 2.1356008 & 5.7846377 \\ \mathrm{~N} & 0.5888743 & 0.8514133 & 3.6472103 \\ \mathrm{C} & -0.8981268 & 1.8077665 & 4.8968004 \\ \mathrm{H} & -1.0846020 & 0.1494293 & 2.6117514 \\ \mathrm{H} & 0.9111819 & -0.7016693 & 1.3167468 \\ \mathrm{H} & -4.9554588 & 2.1085131 & 6.9067717 \\ \mathrm{H} & 0.7970500 & 2.4858747 & 5.9840955 \\ \mathrm{H} & -0368272 & 2.9260616 & 5.4184411 \\ \mathrm{H} & 1.7627726 & 2.5890148\end{array}$




$\begin{array}{lrrr}\mathrm{H} & 1.8480517 & 1.2372638 & 1.9870154 \\ \mathrm{H} & 0.0419364 & 4.7554665 & 6.5597157 \\ \mathrm{H} & -1.5387976 & 4.4760130 & 5.7830544 \\ \mathrm{H} & 1.6124178 & 2.5808945 & 3.1003754 \\ \mathrm{H} & 0.4023410 & 3.4446405 & 4.5531383 \\ \mathrm{H} & -0.4783696 & 1.5420396 & 1.0992826 \\ \mathrm{C} & 0.0206439 & 2.3443666 & 1.6623321 \\ \mathrm{H} & -0.7584198 & 2.8535200 & 2.2490763 \\ \mathrm{C} & 0.6498852 & 3.3356593 & 0.6801069 \\ \mathrm{H} & 1.4209658 & 2.8473127 & 0.0657152 \\ \mathrm{H} & 1.1243366 & 4.1734435 & 1.2123560 \\ \mathrm{H} & -0.1082696 & 3.7509602 & 0.0026505\end{array}$

$$
\mathrm{TS}\left(1+\mathrm{NPr}_{4}{ }^{+} \longrightarrow 1+\mathrm{H}^{+}+\text {Propene }+\mathrm{NPr}_{3}\right)
$$

48

\begin{tabular}{lrrr}
\multicolumn{4}{l}{ Energy $=-757.7084227650$} \\
$\mathrm{H}$ & 0.9425568 & -0.4867420 & 4.0963747 \\
$\mathrm{H}$ & 2.2448045 & 3.4560065 & 1.3674096 \\
$\mathrm{C}$ & -0.1191822 & -0.2731262 & 3.9021681 \\
$\mathrm{H}$ & -0.6938608 & -0.5929829 & 4.7818478 \\
$\mathrm{H}$ & -0.2298068 & 0.8166259 & 3.8014635 \\
$\mathrm{H}$ & 1.2776555 & -0.7979721 & 1.5943942 \\
$\mathrm{C}$ & 1.6680575 & 4.0495583 & 0.6423482 \\
$\mathrm{H}$ & 1.2221577 & -3.1321101 & 1.4206188 \\
$\mathrm{H}$ & 1.1018280 & 4.7993564 & 1.2104466 \\
$\mathrm{H}$ & 2.3620581 & 4.5436117 & -0.0465086 \\
$\mathrm{C}$ & 0.2142070 & -0.5441746 & 1.4340557 \\
$\mathrm{H}$ & 1.4378750 & -5.4755737 & 0.5545187 \\
$\mathrm{C}$ & -0.6029406 & -0.9930496 & 2.6404048 \\
$\mathrm{H}$ & 1.7545777 & -0.7301394 & -0.5918626 \\
$\mathrm{H}$ & 1.6567329 & -3.1242424 & -0.2898569 \\
$\mathrm{C}$ & 0.8502221 & -3.3799537 & 0.4146621
\end{tabular}




$\begin{array}{lrrr}\mathrm{H} & -0.5173232 & -2.0800788 & 2.7864235 \\ \mathrm{H} & 0.1629804 & 0.5498939 & 1.3425704 \\ \mathrm{C} & 0.5446004 & -4.8765695 & 0.3309466 \\ \mathrm{C} & 0.7301546 & 3.1266807 & -0.1135856 \\ \mathrm{C} & 0.7127341 & -0.5661831 & -0.9214922 \\ \mathrm{O} & -0.2670426 & 2.6806708 & 0.6203750 \\ \mathrm{H} & 2.4636815 & -0.4508313 & -3.0578405 \\ \mathrm{H} & 0.5576208 & 0.5228514 & -0.9529420 \\ \mathrm{~N} & -0.2041507 & -1.0868190 & 0.1201413 \\ \mathrm{H} & -0.2404639 & -5.1601204 & 1.0478624 \\ \mathrm{H} & -1.6691909 & -0.7757042 & 2.4807907 \\ \mathrm{C} & -0.3955925 & -2.5497532 & 0.1015065 \\ \mathrm{C} & 1.3983126 & -0.3715508 & -3.3215441 \\ \mathrm{O} & 0.9210489 & 2.8203257 & -1.2983343 \\ \mathrm{H} & 0.1955489 & -5.1525536 & -0.6753980 \\ \mathrm{C} & 0.5273271 & -1.1378611 & -2.3228793 \\ \mathrm{H} & -1.1943299 & -2.7857177 & 0.8190933 \\ \mathrm{H} & 1.1346413 & 0.6957951 & -3.3279902 \\ \mathrm{H} & 0.7913898 & -2.2056871 & -2.3478187 \\ \mathrm{H} & -0.9612825 & 1.8677361 & 0.0770513 \\ \mathrm{H} & -0.7832650 & -2.8171099 & -0.8914435 \\ \mathrm{H} & 1.2718314 & -0.7666303 & -4.3386481 \\ \mathrm{H} & -2.0231270 & -0.3252553 & -0.3153282 \\ \mathrm{H} & -2.4710060 & -0.7747493 & 0.5716464 \\ \mathrm{C} & -2.0815625 & 1.0745455 & -0.4782029 \\ \mathrm{H} & -0.5276182 & -1.0656001 & -2.6264748 \\ \mathrm{H} & -2.7247605 & 1.5630592 & 0.2685479 \\ \mathrm{H} & -2.0823689 & -0.9705074 & -1.1956868 \\ \mathrm{H} & -2.1788292 & 1.6505820 & -1.8864032 \\ \mathrm{H} & -1.4813573 & 1.1397391 & -2.5659513 \\ \mathrm{H} & -12275 & 1.5549136 & -2.3160137\end{array}$




\subsubsection{Deprotonated Amide Catalysis}

\section{Compound $12+\mathrm{NPr}_{4}{ }^{+}$}

58

Energy $=-855.8434039712$

$\begin{array}{lrrr}\mathrm{H} & 4.8729426 & 1.3088301 & 2.4943547 \\ \mathrm{H} & 6.7871099 & -0.2020017 & 7.6875163 \\ \mathrm{H} & 5.2370288 & 0.4065775 & 5.8361835 \\ \mathrm{H} & 3.6316516 & 0.8679179 & 4.6166540 \\ \mathrm{C} & 4.2024712 & 0.5843676 & 2.0100310 \\ \mathrm{H} & 4.3560272 & 0.2444823 & 7.3621968 \\ \mathrm{H} & 3.3140000 & 1.1276520 & 1.6572003 \\ \mathrm{H} & 4.7189483 & 0.1736897 & 1.1327754 \\ \mathrm{C} & 6.1416827 & -0.9668147 & 7.2364742 \\ \mathrm{C} & 4.9105029 & -0.3163930 & 6.5965786 \\ \mathrm{H} & 0.3085305 & 1.8821108 & 1.1583512 \\ \mathrm{H} & 6.7339904 & -1.5141375 & 6.4890323 \\ \mathrm{H} & 2.4563058 & 0.7214611 & 6.5826401 \\ \mathrm{H} & 1.2371664 & -1.7575897 & 0.7312954 \\ \mathrm{C} & 3.0593644 & 0.0510179 & 4.1608161 \\ \mathrm{H} & 4.7189569 & -1.0676017 & 3.3035994 \\ \mathrm{H} & 5.8512630 & -1.6753021 & 8.0256066 \\ \mathrm{C} & 3.8119358 & -0.5371341 & 2.9780802 \\ \mathrm{H} & 2.0854317 & 0.4672858 & 3.8566915 \\ \mathrm{O} & \mathbb{0 . 1 0 1 7 4 4 0} & 1.3567672 & 4.0476105 \\ \mathrm{H} & 1.0691332 & 0.8527164 & 8.7011827 \\ \mathrm{H} & -2.5052200 & 1.4537670 & 4.7616035 \\ \mathrm{C} & 0.2084388 & 0.7965441 & 1.3688684 \\ \mathrm{H} & 1.0307849 & 0.2389949 & 5.6597705 \\ \mathrm{C} & 1.8651243 & -0.1472137 & 6.2667574 \\ \mathrm{C} & -0.9059652 & 0.8382706 & 3.4334423 \\ & 4.0328030 & -1.3928148 & 5.9773887 \\ & 1.1292713 & 0.5280184 & 1.9251037 \\ & & & \\ \mathrm{H} & & \end{array}$




$\begin{array}{rrrr}\text { N } & 2.7563885 & -0.9160530 & 5.2995191 \\ \mathrm{~N} & -0.9861055 & 0.5240891 & 2.1545197 \\ \mathrm{C} & 0.2832201 & -1.4864007 & 0.2512271 \\ \mathrm{H} & 4.5886503 & -1.9527899 & 5.2155143 \\ \mathrm{H} & -0.3651320 & 0.3554308 & 7.7751393 \\ \mathrm{H} & 3.1822867 & -1.2684741 & 2.4503845 \\ \mathrm{C} & 0.5000162 & -0.0171991 & 8.3425484 \\ \mathrm{C} & -2.1388421 & 0.5346458 & 4.2779153 \\ \mathrm{H} & 1.0266466 & 0.3705623 & -0.5853847 \\ \mathrm{H} & -0.5261339 & -1.8307713 & 0.9100623 \\ \mathrm{C} & 0.1905706 & 0.0262904 & 0.0462643 \\ \mathrm{H} & 3.7158581 & -2.1141280 & 6.7401684 \\ \mathrm{H} & 0.2175220 & -2.0368172 & -0.6988387 \\ \mathrm{H} & -2.9468973 & 0.0925177 & 3.6810979 \\ \mathrm{C} & 1.3741436 & -0.9282096 & 7.4739463 \\ \mathrm{H} & 2.2153551 & -1.3070766 & 8.0729024 \\ \mathrm{H} & 0.1240131 & -0.5623290 & 9.2180096 \\ \mathrm{H} & -1.8693222 & -0.1634776 & 5.0863759 \\ \mathrm{H} & 0.8086947 & -1.2446536 & 3.2750224 \\ \mathrm{C} & 2.0620697 & -2.1681004 & 4.7870193 \\ \mathrm{H} & -0.7392023 & 0.2721421 & -0.4950339 \\ \mathrm{H} & 2.7726054 & -2.6442388 & 4.1004455 \\ \mathrm{H} & 0.0102240 & -1.4949548 & 4.8186335 \\ \mathrm{H} & 0.7845968 & -1.8009284 & 7.1577427 \\ \mathrm{C} & 0.7206584 & -1.9465223 & 4.1136819 \\ \mathrm{H} & 1.9589236 & -2.8313060 & 5.6545162 \\ \mathrm{C} & 0.1645356 & -3.2801774 & 3.6118979 \\ \mathrm{H} & 0.8342695 & -3.7371617 & 2.8689259 \\ \mathrm{H} & -0.8124429 & -3.1258017 & 3.1362357 \\ & 0.0337759 & -3.9957121 & 4.4370705 \\ & & & \end{array}$




\section{Compound $12+\mathrm{Pr}^{+}$}

\begin{tabular}{lrrr}
27 & \multicolumn{3}{l}{} \\
Energy $=-445.3223848169$ & \\
$\mathrm{O}$ & 1.5839164 & 0.0483710 & 1.5689820 \\
$\mathrm{H}$ & -1.9110001 & 0.9155163 & 0.2086526 \\
$\mathrm{H}$ & -4.2107769 & -0.1862961 & -0.2232084 \\
$\mathrm{H}$ & -0.1236671 & 0.6958154 & 3.2618447 \\
$\mathrm{C}$ & 0.3904323 & 0.2068275 & 1.2632855 \\
$\mathrm{C}$ & -1.4497533 & 0.1398152 & -0.4022077 \\
$\mathrm{C}$ & -0.6102565 & 0.6952861 & 2.2888075 \\
$\mathrm{C}$ & -3.6953702 & -0.9406209 & -0.8248539 \\
$\mathrm{~N}$ & -0.0583126 & -0.0393749 & 0.0130822 \\
$\mathrm{H}$ & -1.4369047 & 0.5137750 & -1.4297507 \\
$\mathrm{H}$ & -0.9321554 & 1.7136349 & 2.0544541 \\
$\mathrm{C}$ & -2.2661086 & -1.1510334 & -0.3297041 \\
$\mathrm{H}$ & -2.2745494 & -1.5102454 & 0.7040438 \\
$\mathrm{H}$ & -3.7025341 & -0.6028257 & -1.8651735 \\
$\mathrm{H}$ & 2.0717028 & 1.2717848 & -1.0776072 \\
$\mathrm{H}$ & 0.7395070 & 1.2575616 & -2.2293886 \\
$\mathrm{H}$ & -4.2695705 & -1.8679425 & -0.7670744 \\
$\mathrm{H}$ & -1.5000895 & 0.0644450 & 2.3292564 \\
$\mathrm{C}$ & 1.5248792 & 0.6391472 & -1.7826278 \\
$\mathrm{C}$ & 0.8798478 & -0.5093154 & -1.0073096 \\
$\mathrm{H}$ & -1.7722892 & -1.9218088 & -0.9294999 \\
$\mathrm{H}$ & 1.6464434 & -1.1059198 & -0.5125349 \\
$\mathrm{H}$ & 0.3256779 & -1.1604817 & -1.6882763 \\
$\mathrm{C}$ & 2.4649078 & 0.1262396 & -2.8715900 \\
$\mathrm{H}$ & 2.9215207 & 0.9549169 & -3.4176726 \\
$\mathrm{H}$ & 3.2690457 & -0.4779692 & -2.4413745 \\
$\mathrm{H}$ & 1.9261871 & -0.4963691 & -3.5920542
\end{tabular}




\section{Compound $12+\mathrm{H}^{+}$}

18

Energy $=-327.3282306066$

$\begin{array}{lrrr}\text { C } & -2.3417258 & 0.4474555 & 4.0840354 \\ \mathrm{C} & -1.2926191 & 0.9574020 & 3.1230298 \\ \mathrm{~N} & -0.6506553 & 0.0160655 & 2.4041891 \\ \mathrm{C} & 0.3602644 & 0.3005913 & 1.3943442 \\ \mathrm{C} & -0.1170790 & -0.0382260 & -0.0191570 \\ \mathrm{C} & -1.3120890 & 0.7998663 & -0.4675391 \\ \mathrm{O} & -1.0496768 & 2.1675380 & 3.0195627 \\ \mathrm{H} & 0.5931153 & 1.3624049 & 1.4780991 \\ \mathrm{H} & 1.2612395 & -0.2703763 & 1.6336373 \\ \mathrm{H} & -3.3035396 & 0.8917381 & 3.8193355 \\ \mathrm{H} & -0.3684716 & -1.1035445 & -0.0650638 \\ \mathrm{H} & 0.7261263 & 0.1150028 & -0.7001564 \\ \mathrm{H} & -2.0868281 & 0.7799639 & 5.0922534 \\ \mathrm{H} & -2.4342405 & -0.6389761 & 4.0756542 \\ \mathrm{H} & -2.1624543 & 0.6603186 & 0.2051236 \\ \mathrm{H} & -1.0608862 & 1.8641462 & -0.4753687 \\ \mathrm{H} & -1.6305423 & 0.5176857 & -1.4739115 \\ \mathrm{H} & -0.9181809 & -0.9471758 & 2.5437175\end{array}$

\section{$\mathrm{TS}\left(12+\mathrm{NPr}_{4}{ }^{+} \longrightarrow\right.$ 12+Pr $\left.{ }^{+}+\mathrm{NPr}_{3}\right)$}

58

Energy $=-855.1777449752$

$\begin{array}{lrrr}\mathrm{H} & 7.8749556 & -0.2592273 & -2.1846818 \\ \mathrm{H} & 7.2686887 & 1.2190319 & -1.4296017 \\ \mathrm{C} & 7.3342586 & 0.1384973 & -1.3244455 \\ \mathrm{H} & 6.5647006 & 3.3906783 & -1.1668261 \\ \mathrm{O} & 4.4301992 & 3.3895238 & -2.8774304 \\ \mathrm{H} & 3.7207103 & 0.8652803 & -3.5831409 \\ \mathrm{H} & 7.9224594 & -0.0860894 & -0.4314743\end{array}$




\begin{tabular}{|c|c|c|c|}
\hline & 5.3856418 & -0.2265324 & -2.1577955 \\
\hline & 4.2699591 & 2.3888298 & -2.1553207 \\
\hline & 5.9340543 & -0.4635249 & -1.2497386 \\
\hline & 7.7530071 & 4.5596802 & 0.8297069 \\
\hline & 5.6830930 & 3.2619184 & -0.5178151 \\
\hline & 3.3131958 & 1.3109544 & -2.6719848 \\
\hline & 4.8364000 & 2.1706911 & -0.9675426 \\
\hline & 6.0139307 & -1.5552693 & -1.2353059 \\
\hline & 5.1406114 & 4.2129218 & -0.6027452 \\
\hline & 3.0995095 & 0.5186344 & -1.9520377 \\
\hline & 6.8829757 & 4.3280034 & 1.4492947 \\
\hline$F$ & 6.8266193 & 2.2315052 & 1.0037590 \\
\hline ( & 6.1534857 & 3.0938124 & 0.9249525 \\
\hline F & 2.3733128 & 1.7911666 & -2.9476476 \\
\hline$c$ & 5.1193731 & 0.0125162 & -0.0753670 \\
\hline $\mathrm{f}$ & 6.2282560 & 5.2022725 & 1.4325856 \\
\hline F & 9.0941175 & -1.5203523 & 1.5966159 \\
\hline$H$ & 7.2313985 & 4.1892684 & 2.4757432 \\
\hline $\mathrm{H}$ & 6.8800164 & -2.1419645 & 0.6347299 \\
\hline F & 5.5719980 & 0.6562362 & 0.6492633 \\
\hline F & 2.9950309 & -3.9451401 & -1.5512601 \\
\hline $\mathrm{H}$ & 4.9577394 & -2.9488095 & -0.3164893 \\
\hline $\mathrm{H}$ & 4.0612644 & 0.1374701 & -0.1793761 \\
\hline$H$ & 7.1871274 & 0.0777210 & 1.854 \\
\hline $\mathrm{H}$ & 5.2856667 & 2.8769278 & 1.5585252 \\
\hline$c$ & 8.5829925 & -1.4009839 & 2.5542611 \\
\hline $\mathrm{H}$ & 2.9426786 & -1.5495722 & -0.8096662 \\
\hline C & 6.3425118 & -1.8896041 & 1.5490716 \\
\hline$C$ & 7.1574227 & -0.8889767 & 2.3574994 \\
\hline $\mathrm{H}$ & 9.1642492 & -0.7030723 & 3.1570681 \\
\hline C & 2.3611259 & -3.6185554 & -0.7240064 \\
\hline C & 4.3049710 & -2.6080666 & 0.4873501 \\
\hline & 4.9845761 & -1.4509305 & 1.12752 \\
\hline
\end{tabular}




$\begin{array}{lrrr}\mathrm{C} & 2.9128975 & -2.3517811 & -0.0721987 \\ \mathrm{H} & 1.3613592 & -3.4436427 & -1.1215451 \\ \mathrm{H} & 8.5921658 & -2.3686781 & 3.0614699 \\ \mathrm{H} & 6.2444700 & -2.8195802 & 2.1191502 \\ \mathrm{H} & 2.2970403 & -4.4400247 & -0.0064434 \\ \mathrm{H} & 4.2635933 & -3.4252331 & 1.2146687 \\ \mathrm{H} & 6.6966110 & -0.7233657 & 3.3332456 \\ \mathrm{C} & 4.1925111 & -0.8635215 & 2.2390296 \\ \mathrm{H} & 2.2338677 & -2.0306404 & 0.7201911 \\ \mathrm{H} & 4.7427087 & 0.0050732 & 2.5963463 \\ \mathrm{H} & 3.2708198 & -0.4776499 & 1.8067278 \\ \mathrm{C} & 3.8716655 & -1.7917930 & 3.4045920 \\ \mathrm{H} & 4.7905940 & -2.2154561 & 3.8169990 \\ \mathrm{H} & 3.2648084 & -2.6335728 & 3.0631410 \\ \mathrm{C} & 3.1219349 & -1.0371777 & 4.5009372 \\ \mathrm{H} & 3.7200963 & -0.2101951 & 4.8896264 \\ \mathrm{H} & 2.1858612 & -0.6202202 & 4.1232330 \\ \mathrm{H} & 2.8810307 & -1.6966983 & 5.3351406\end{array}$

$$
\mathrm{TS}\left(12+\mathrm{NPr}_{4}{ }^{+} \longrightarrow 12+\mathrm{H}^{+}+\text {Propene }+\mathrm{NPr}_{3}\right)
$$

\begin{tabular}{lrrr}
58 & \multicolumn{3}{l}{} \\
Energy $=-855.8084708102$ & \\
$\mathrm{H}$ & 5.8108373 & -0.4037652 & 1.7944079 \\
$\mathrm{H}$ & 1.2400639 & 3.3535709 & 2.1180698 \\
$\mathrm{H}$ & 3.5631691 & 0.6676450 & 1.6264505 \\
$\mathrm{H}$ & 3.4154740 & -1.0902298 & 1.5465834 \\
$\mathrm{H}$ & 1.3425775 & 0.8841052 & 1.5660086 \\
$\mathrm{C}$ & 3.7916168 & -0.2097862 & 1.0041782 \\
$\mathrm{C}$ & 5.3071182 & -0.3277901 & 0.8217255 \\
$\mathrm{C}$ & 0.9613401 & 3.5354441 & 1.0698128 \\
$\mathrm{H}$ & 1.1161237 & -1.2500479 & 1.3563697 \\
$\mathrm{H}$ & 0.6583812 & -3.7809110 & 1.3476678
\end{tabular}




$\begin{array}{lrrr}\mathrm{H} & -0.1359854 & 3.5069713 & 1.0042450 \\ \mathrm{H} & 1.2903545 & 4.5479020 & 0.8012936 \\ \mathrm{H} & 5.7119100 & 0.5506725 & 0.2979942 \\ \mathrm{C} & 1.0924206 & 1.1087780 & 0.5205432 \\ \mathrm{H} & 5.5665651 & -1.2205835 & 0.2340910 \\ \mathrm{C} & 1.5945702 & 2.4942229 & 0.1416692 \\ \mathrm{H} & -2.2558467 & 3.8069194 & -0.4613860 \\ \mathrm{C} & 0.9973459 & -1.2926161 & 0.2653904 \\ \mathrm{H} & 2.6899851 & 2.5547134 & 0.2209537 \\ \mathrm{C} & 0.6764958 & -3.7659970 & 0.2480317 \\ \mathrm{H} & 0.0015074 & 1.0670364 & 0.4142485 \\ \mathrm{C} & 3.1051871 & -0.0898900 & -0.3501017 \\ \mathrm{~N} & 1.6061498 & -0.0404278 & -0.3084185 \\ \mathrm{O} & -3.7208461 & 2.1584533 & 0.9832855 \\ \mathrm{H} & -0.3618749 & -3.6772011 & -0.1041955 \\ \mathrm{H} & -0.0725841 & -1.1925899 & 0.0351811 \\ \mathrm{C} & 1.5341644 & -2.6103801 & -0.2748186 \\ \mathrm{H} & 2.5784173 & -2.7608862 & 0.0350762 \\ \mathrm{C} & -2.4755948 & 2.8478341 & -0.9493737 \\ \mathrm{H} & 1.0695893 & -4.7309940 & -0.0977687 \\ \mathrm{H} & -1.4504635 & 0.4294311 & -0.7906950 \\ \mathrm{C} & -2.8760174 & 1.8319100 & 0.1064168 \\ \mathrm{H} & 3.4327270 & 0.8186976 & -0.8719200 \\ \mathrm{H} & -1.6565871 & -0.6333804 & 1.5633159 \\ \mathrm{H} & 3.3706648 & -0.9379797 & -0.9938499 \\ \mathrm{H} & 1.3328312 & 2.7255631 & -0.9003149 \\ \mathrm{H} & -3.3231095 & 3.0128577 & -1.6313019 \\ \mathrm{H} & -2.2873788 & 0.6315265 & 0.0129096 \\ \mathrm{H} & -1.6088056 & 2.5233241 & -1.5365576 \\ \mathrm{H} & 1.5211999 & -2.6107373 & -1.3741487 \\ \mathrm{H} & -1.0748715 & 0.1249497 & -1.8442296 \\ \mathrm{H} & -0.0337608 & 1.6984997\end{array}$




$\begin{array}{lrrr}\mathrm{C} & -0.3891507 & 0.1178268 & -2.0112394 \\ \mathrm{H} & -0.6835029 & 1.0019605 & -2.5970070 \\ \mathrm{H} & 1.5633858 & 1.0607527 & -2.1390558 \\ \mathrm{H} & -2.3448022 & -3.0507833 & 1.8903685 \\ \mathrm{C} & -3.0739410 & -1.7066666 & 0.3367326 \\ \mathrm{H} & 1.6169334 & -0.7048752 & -2.3284426 \\ \mathrm{H} & -2.3523668 & -2.0348006 & -0.4273029 \\ \mathrm{C} & -3.2839685 & -2.8179538 & 1.3652368 \\ \mathrm{H} & -4.0245291 & -2.5235264 & 2.1244744 \\ \mathrm{C} & -0.9529421 & -1.1519703 & -2.6452414 \\ \mathrm{H} & -4.0130370 & -1.4949433 & -0.1992444 \\ \mathrm{H} & -3.6387046 & -3.7427694 & 0.8890550 \\ \mathrm{H} & -2.0461411 & -1.0808604 & -2.7655071 \\ \mathrm{H} & -0.7754225 & -2.0437534 & -2.0193793 \\ \mathrm{H} & -0.5345375 & -1.3940816 & -3.6452919\end{array}$

\subsubsection{Deprotonated Urea Catalysis}

\section{Compound $18+\mathrm{NPr}_{4}{ }^{+}$}

\begin{tabular}{lrrr}
66 & \\
\multicolumn{4}{l}{ Energy $=-989.8998586495$} \\
H & -2.1635725 & -1.7378432 & -3.1470732 \\
$\mathrm{C}$ & -1.3661748 & -1.1058462 & -2.7345897 \\
$\mathrm{H}$ & -0.5364267 & -1.7506094 & -2.4050054 \\
$\mathrm{H}$ & -0.9908350 & -0.4604376 & -3.5423053 \\
$\mathrm{C}$ & -1.8963470 & -0.2625794 & -1.5684874 \\
$\mathrm{H}$ & -2.3702822 & -0.9208529 & -0.8261504 \\
$\mathrm{H}$ & -2.6733922 & 0.4253194 & -1.9322956 \\
$\mathrm{H}$ & -2.9173245 & -1.4671817 & 2.4789087 \\
$\mathrm{H}$ & -3.3164412 & 4.1144415 & -2.1882428 \\
$\mathrm{C}$ & -0.7334354 & 0.4898175 & -0.9468588 \\
$\mathrm{H}$ & -2.7708006 & 0.4047477 & 0.8143623 \\
$\mathrm{H}$ & -2.8923284 & 2.1248691 & -0.6635515
\end{tabular}




$\begin{array}{lrrr}\mathrm{H} & 0.0077081 & -0.2022353 & -0.5192753 \\ \mathrm{H} & -1.1418902 & 2.8438470 & -2.3325081 \\ \mathrm{H} & -0.7257969 & -1.0636828 & 1.2791101 \\ \mathrm{H} & -0.2163653 & 1.0865155 & -1.7073869 \\ \mathrm{C} & -2.0525337 & -1.0222588 & 2.9924485 \\ \mathrm{C} & -2.3713270 & 4.5492031 & -1.8324787 \\ \mathrm{C} & -1.8530976 & 0.7819179 & 1.2809662 \\ \mathrm{C} & -1.9581752 & 2.5975908 & -0.3372579 \\ \mathrm{C} & -1.3709280 & 3.4542586 & -1.4477479 \\ \mathrm{C} & -1.1129638 & -0.3323375 & 2.0006558 \\ \mathrm{~N} & -1.0750944 & 1.4678279 & 0.1697932 \\ \mathrm{O} & 1.6119819 & -2.5658454 & -1.4382977 \\ \mathrm{H} & -1.9621912 & 5.1763944 & -2.6349605 \\ \mathrm{H} & -1.5261656 & -1.8245870 & 3.5261102 \\ \mathrm{H} & 2.0369528 & 0.4649237 & -1.3049221 \\ \mathrm{H} & 0.2206444 & -4.8933006 & 1.1747964 \\ \mathrm{~N} & 1.1062909 & -3.0499818 & 0.7604911 \\ \mathrm{C} & 1.5953245 & -2.1556405 & -0.2307502 \\ \mathrm{H} & 0.5459732 & -4.5513338 & -0.5371548 \\ \mathrm{C} & 0.1766708 & -4.1096245 & 0.3997809 \\ \mathrm{H} & 3.6058649 & 2.4231011 & -1.4374051 \\ \mathrm{H} & -2.4328886 & -0.3121497 & 3.7410558 \\ \mathrm{H} & -0.2410258 & 0.0673386 & 2.5365189 \\ \mathrm{H} & -2.1464410 & 1.5733313 & 1.9807063 \\ \mathrm{H} & -2.1952664 & 3.2125586 & 0.5390745 \\ \mathrm{C} & 2.7362292 & -0.1560569 & -0.7001914 \\ \mathrm{H} & 2.0333269 & -0.9939173 & 0.2543290 \\ \mathrm{H} & 3.1701441 & 1.3283953 & 0.8001878 \\ \mathrm{H} & 0.9219537 & -2.5836608 & 1.6467096 \\ \mathrm{H} & -0.4304842 & 3.9210411 & -1.1213107 \\ \mathrm{H} & -2596704 & 1.9855215 & 0.6848140\end{array}$




$\begin{array}{lrrr}\text { C } & 4.3644656 & 1.7929854 & -0.9466238 \\ \mathrm{H} & 0.8304521 & 1.0888554 & 0.9683626 \\ \mathrm{H} & 0.7652498 & 2.4224280 & -0.1852832 \\ \mathrm{H} & 3.2891621 & -0.7593674 & -1.4489219 \\ \mathrm{H} & -2.1862015 & -5.6309440 & 0.5043831 \\ \mathrm{C} & -1.2793191 & -3.6687937 & 0.2037960 \\ \mathrm{H} & 5.0608483 & 2.4602857 & -0.4185207 \\ \mathrm{H} & -1.8444096 & -5.2413005 & -1.1955135 \\ \mathrm{C} & -2.1854661 & -4.8180874 & -0.2382560 \\ \mathrm{H} & 4.9292125 & 1.2797621 & -1.7405430 \\ \mathrm{C} & 0.1779712 & 2.9935689 & 1.8190446 \\ \mathrm{H} & -1.6577373 & -3.2355060 & 1.1437246 \\ \mathrm{H} & 4.4819962 & 0.1899382 & 0.5158177 \\ \mathrm{H} & -0.3525905 & 2.5650274 & 2.6813015 \\ \mathrm{H} & -0.3823887 & 3.8867950 & 1.5069772 \\ \mathrm{H} & -1.2972507 & -2.8671721 & -0.5493743 \\ \mathrm{H} & -3.2244575 & -4.4841107 & -0.3701543 \\ \mathrm{C} & 1.5896258 & 3.4051562 & 2.2479177 \\ \mathrm{H} & 2.1591504 & 2.5405920 & 2.6175809 \\ \mathrm{H} & 2.1476951 & 3.8463581 & 1.4093398 \\ \mathrm{H} & 1.5440144 & 4.1500434 & 3.0527874\end{array}$

\section{Compound $18+\mathrm{Pr}^{+}$}

35

\begin{tabular}{lrrr}
\multicolumn{4}{l}{ Energy $=-579.3762503135$} \\
$\mathrm{H}$ & 1.4386404 & -3.1245938 & 2.0498644 \\
$\mathrm{H}$ & 1.4402343 & -5.4611264 & 3.0037891 \\
$\mathrm{H}$ & -0.5512989 & -1.0059157 & -1.9926375 \\
$\mathrm{H}$ & 0.2016079 & 0.5299867 & -2.3758316 \\
$\mathrm{O}$ & 0.1096092 & -0.5359095 & 1.8217014 \\
$\mathrm{H}$ & 0.6206488 & -2.1016784 & -4.0248149 \\
$\mathrm{C}$ & 0.5007540 & -4.9637231 & 2.7252116
\end{tabular}




$\begin{array}{rrrr}\mathrm{C} & 0.3521764 & -0.4023258 & -1.8114951 \\ \mathrm{C} & 0.7362259 & -3.8826044 & 1.6695096 \\ \mathrm{H} & 0.0635980 & -4.5346457 & 3.6396940 \\ \mathrm{H} & -0.9404357 & 2.0628722 & 0.6941424 \\ \mathrm{H} & 1.1977083 & -4.3251600 & 0.7715694 \\ \mathrm{H} & 1.3501908 & -0.5307795 & -4.4231990 \\ \mathrm{C} & \mathbb{0 . 0 6 9 4 7 0 9} & -0.8895472 & 0.6236442 \\ \mathrm{C} & 1.4833881 & -1.4471803 & -3.8286544 \\ \mathrm{~N} & \mathbb{0 . 4 3 3 2 6 9 0} & -0.0458473 & -0.3964613 \\ \mathrm{C} & 1.5996346 & -1.1221686 & -2.3393175 \\ \mathrm{H} & -0.1909855 & -5.7357363 & 2.3548372 \\ \mathrm{C} & -0.1878405 & 2.3516500 & -0.0560131 \\ \mathrm{C} & -0.5646419 & -3.1803992 & 1.2652382 \\ \mathrm{~N} & -0.3830771 & -2.1408989 & 0.2586240 \\ \mathrm{H} & -1.0267321 & -2.7137872 & 2.1456277 \\ \mathrm{C} & \mathbb{0 . 9 1 8 1 7 5 9} & 1.2921771 & -0.0644151 \\ \mathrm{H} & 1.3981396 & 1.2469068 & 0.9217856 \\ \mathrm{H} & 2.3838792 & -1.9587160 & -4.1952044 \\ \mathrm{H} & -\mathbb{0} .6965330 & 2.3538213 & -1.0338598 \\ \mathrm{H} & 1.7624995 & -2.0467146 & -1.7620180 \\ \mathrm{H} & -\mathbb{0} .1764991 & -2.4681031 & -0.6791472 \\ \mathrm{C} & \mathbb{0 . 3 5 9 4 5 3 9} & 3.7464234 & 0.2501385 \\ \mathrm{H} & -0.4427688 & 4.4973633 & 0.2563293 \\ \mathrm{H} & \mathbb{0 . 8 5 0 8 6 9 6} & 3.7714390 & 1.2347669 \\ \mathrm{H} & 2.4784101 & -0.4839432 & -2.1559478 \\ \mathrm{H} & -1.2799304 & -3.9129633 & 0.8619656 \\ \mathrm{H} & 1.6927921 & 1.5601643 & -0.8003403 \\ & 1.1022100 & 4.0541809 & -0.5016688\end{array}$

\section{Compound $18+\mathrm{H}^{+}$}

26

Energy $=-461.3895322453$ 


\begin{tabular}{|c|c|c|c|}
\hline $\mathrm{H}$ & 0.6719043 & -3.8034067 & 1.5628472 \\
\hline $\mathrm{H}$ & -0.7096833 & -1.8934053 & 0.4804405 \\
\hline C & 1.3665913 & -3.0360047 & 1.9372226 \\
\hline $\mathrm{H}$ & 1.7516346 & -2.1157239 & 0.0093841 \\
\hline C & 0.0160963 & -1.1666173 & 0.8752012 \\
\hline $\mathrm{H}$ & 2.3605782 & -3.4967295 & 2.0229836 \\
\hline C & 1.3960428 & -1.8200833 & 1.0099365 \\
\hline $\mathrm{H}$ & 1.3346802 & 3.6584473 & 0.7438804 \\
\hline $\mathrm{H}$ & 0.1301249 & 5.8965271 & 1.2550493 \\
\hline $\mathrm{N}$ & -0.0015030 & -0.0004894 & 0.0004028 \\
\hline 0 & 0.5299542 & 1.4857858 & 1.6720043 \\
\hline $\mathrm{H}$ & -1.1651847 & 3.7847641 & 0.7374965 \\
\hline 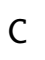 & 0.4014002 & 1.2326390 & 0.4585145 \\
\hline $\mathrm{H}$ & 0.0881510 & -0.1804260 & -0.9962394 \\
\hline $\mathrm{H}$ & 1.0384598 & -2.7536272 & 2.9491572 \\
\hline $\mathrm{N}$ & 0.6519709 & 2.1659682 & -0.5206166 \\
\hline $\mathrm{H}$ & -0.3424456 & -0.8432903 & 1.8622188 \\
\hline C & 0.7662572 & 3.5820002 & -0.1933552 \\
\hline$c$ & -0.5819860 & 4.2957802 & -0.0448551 \\
\hline C & -0.4117730 & 5.7749864 & 0.3047850 \\
\hline $\mathrm{H}$ & 0.3570696 & 1.9413726 & -1.4673503 \\
\hline $\mathrm{H}$ & -1.3846772 & 6.2757022 & 0.4065701 \\
\hline $\mathrm{H}$ & 2.1047264 & -1.0680551 & 1.3913738 \\
\hline $\mathrm{H}$ & 1.3634568 & 4.0618199 & -0.9833130 \\
\hline $\mathrm{H}$ & Q. 1581594 & 6.3036575 & -0.4745379 \\
\hline $\mathrm{H}$ & -1.1482747 & 4.1917267 & -0.98477 \\
\hline
\end{tabular}

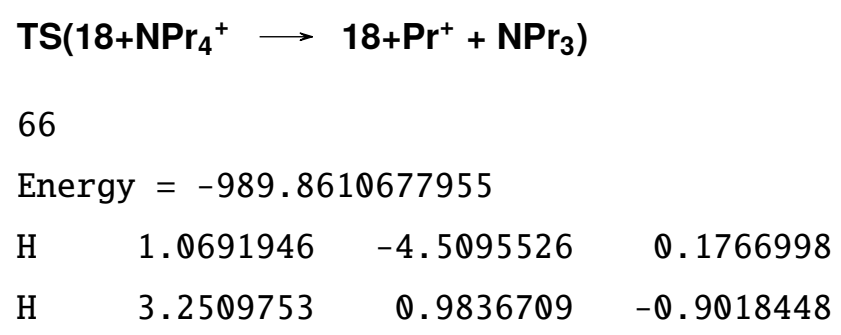




\begin{tabular}{|c|c|c|c|}
\hline & 1.5958108 & -2.2934208 & 833142 \\
\hline & 3.3072771 & 1.7330801 & 3.1351974 \\
\hline & 2.2838842 & -0.3213457 & 2.1377397 \\
\hline & 0.1630038 & -5.0047477 & 1.6240667 \\
\hline & 1.9096303 & -0.8581111 & 0.0201034 \\
\hline & 2.7867932 & 1.1241571 & 4.7218702 \\
\hline & 0.0824955 & -4.4778186 & 0.6618081 \\
\hline & 2.4797968 & 1.7352245 & 3.8602039 \\
\hline & 1.5957193 & -0.8842000 & 3.6604166 \\
\hline & 0.7057212 & -2.7934590 & 2.7153733 \\
\hline & 2.2790961 & 1.2164159 & -0.4457518 \\
\hline C & 0.5975982 & -2.2743049 & 1.7462751 \\
\hline ( & 1.3920646 & -0.2503229 & 2.7786972 \\
\hline & 2.4686933 & 1.5758005 & 0.5759109 \\
\hline & 1.3758860 & -0.0168476 & -0.4529481 \\
\hline & -0.6237158 & -5.0329549 & 0.0301711 \\
\hline & 1.1856819 & -0.3561429 & -1.4819315 \\
\hline$C$ & -0.3859228 & -3.0346203 & 0.863 \\
\hline 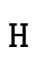 & 2.0 & 64103 & 4.2098992 \\
\hline & 1.0728722 & -2.2141906 & -3.5257690 \\
\hline ( & 1.1950077 & 1.1923852 & 3.2308324 \\
\hline $\mathrm{N}$ & 0.2751660 & -0.8498133 & 2.0052552 \\
\hline $\mathrm{H}$ & 1.8237924 & 2.0372944 & -1.013 \\
\hline $\mathrm{H}$ & 1.8919487 & -2.5195695 & -5.8992354 \\
\hline $\mathrm{H}$ & -0.4758149 & -2.5411816 & -0.1155320 \\
\hline$c$ & 0.0521103 & 0.1741549 & 0.2211877 \\
\hline C & 0.7084535 & -1.5582517 & -4.3330876 \\
\hline 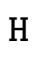 & 0.9114901 & 1.8274685 & 2.3778467 \\
\hline C & 0.8464352 & -2.2545423 & -5.6874098 \\
\hline $\mathrm{H}$ & 1.3269178 & -0.6475936 & -4.3076100 \\
\hline $\mathrm{H}$ & -0.4818709 & -0.9431422 & 4.6978096 \\
\hline $\mathrm{H}$ & -1.3894678 & -3.0364539 & 1.3133161 \\
\hline $\mathrm{H}$ & -1.1374788 & -2.3869313 & 3.9212039 \\
\hline
\end{tabular}




\begin{tabular}{|c|c|c|c|}
\hline & 0.3745677 & 1.2581870 & 3.9599958 \\
\hline & 0.2525445 & -3.1812148 & -5.7210252 \\
\hline & -1.2551641 & -1.2955317 & 3.9979676 \\
\hline & -1.0548558 & -0.6500402 & 2.6269360 \\
\hline & -0.7888425 & -0.4631545 & -0.0089302 \\
\hline & -0.1653806 & 1.0645740 & 0.7911295 \\
\hline & -0.8886209 & -0.4558762 & -2.7665875 \\
\hline & -0.7400034 & -1.1565762 & -4.0287705 \\
\hline & -0.6940836 & 0.9217184 & -2.6503171 \\
\hline & 0.4937485 & -1.6044140 & -6.5030133 \\
\hline & -1.3748394 & -2.0550517 & -3.9930152 \\
\hline & -1.4180240 & -0.9055137 & -2.0297011 \\
\hline & -0.9047428 & 1.4204280 & -1.4165127 \\
\hline & -1.8072577 & -1.0301816 & 1.9224271 \\
\hline & -1.2205068 & 0.4338033 & 2.6974856 \\
\hline & -0.1419182 & 3.0485571 & 0.7492646 \\
\hline & 0.2448889 & 3.2059739 & -1.6851717 \\
\hline & -0.3400543 & 1.5730959 & -3.6785602 \\
\hline & -2.6419970 & -0.9652220 & 4.5531889 \\
\hline & -0.7473062 & 2.8645780 & -1.3225293 \\
\hline & -2.7940576 & -1.4309928 & 5.5362426 \\
\hline & -0.9607629 & 3.3934494 & 0.0964127 \\
\hline & -1.1124817 & -0.5187079 & -4.8478722 \\
\hline & -2.7721729 & 0.1210955 & 4.6695796 \\
\hline & -3.4335597 & -1.3282604 & 3.8808255 \\
\hline & -0.0934927 & 5.3621414 & -0.2637008 \\
\hline & -1.8907748 & 2.9651596 & 0.5057840 \\
\hline & -1.0165111 & 4.9210769 & 0.1433067 \\
\hline & -1.4792395 & 3.3657215 & -1.9893624 \\
\hline & -1.1370240 & 5.2887694 & 1.1722679 \\
\hline & -1.8590550 & 5.3037273 & -0.4528412 \\
\hline
\end{tabular}




\begin{tabular}{|c|c|c|c|}
\hline \multicolumn{4}{|c|}{$\mathrm{TS}\left(18+\mathrm{NPr}_{4}{ }^{+} \longrightarrow 18+\mathrm{H}^{+}+\right.$Propene $\left.+\mathrm{NPr}_{3}\right)$} \\
\hline \multicolumn{4}{|c|}{66} \\
\hline \multicolumn{4}{|c|}{ Energy $=-989.8647567080$} \\
\hline$H$ & 5.7511924 & -0.3908088 & 1.7550900 \\
\hline U & 3.4529731 & 0.5914188 & 1.6637269 \\
\hline & 5.2419634 & -0.2560973 & 0.7917528 \\
\hline 14 & 1.1878584 & 3.2429644 & 2.2480756 \\
\hline $\mathbf{\Pi}$ & 3.3937903 & -1.1622745 & 1.4605066 \\
\hline$C$ & 3.7228800 & -0.2278445 & 0.9818090 \\
\hline $\mathrm{I}$ & 5.6018961 & 0.6825945 & 0.3457338 \\
\hline & 1.2767152 & 0.8257577 & 1.5897139 \\
\hline 11 & 5.5427553 & -1.0816788 & 0.1301955 \\
\hline E & 0.8524615 & 3.4701435 & 1.2254367 \\
\hline $\mathrm{H}$ & 0.8858659 & -3.8477233 & 1.3157119 \\
\hline $\mathrm{H}$ & 1.0957715 & -1.3061882 & 1.3111713 \\
\hline 11 & 1.1472728 & 4.5016814 & 0.9920030 \\
\hline$c$ & 0.9969389 & 1.0803956 & 0.5589305 \\
\hline $\mathrm{NA}$ & 2.5541717 & 2.5679592 & 0.2435918 \\
\hline 14 & -0.2451076 & 3.4198952 & 1.2060161 \\
\hline 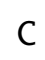 & 1.4574426 & 2.4888914 & 0.2174851 \\
\hline 1 & -1.7186949 & -0.6874547 & 1.7066205 \\
\hline$C$ & 0.7436918 & -3.7984159 & 0.2263477 \\
\hline 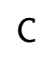 & 3.0303249 & -0.0478631 & -0.3618505 \\
\hline 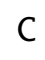 & 0.9486220 & -1.3131962 & 0.2230283 \\
\hline n & -2.2595337 & 4.7935182 & 0.9080900 \\
\hline $\mathrm{H}$ & -2.2847439 & -3.1249246 & 2.0616424 \\
\hline $\mathrm{H}$ & 2.5654945 & -2.7076224 & -0.1703445 \\
\hline 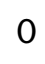 & -3.7814907 & 2.0336687 & 1.0525491 \\
\hline & -0.0950772 & 1.0141664 & 0.4760790 \\
\hline IV & 1.5273567 & -0.0284820 & -0.3171445 \\
\hline & 1.4901992 & -2.6036935 & -0.3743355 \\
\hline & -3.4229229 & -0.2113685 & 1.7012408 \\
\hline
\end{tabular}




\begin{tabular}{|c|c|c|c|}
\hline & -2.5986881 & -0.5383890 & 1.0473914 \\
\hline & -2.3776199 & 7.2098102 & 0.1789353 \\
\hline & 1.1057474 & -4.7399863 & -0.2067165 \\
\hline & -0.3365230 & -3.7299230 & 0.0308163 \\
\hline & 3.3390830 & 0.8936277 & -0.8333510 \\
\hline & -0.1279540 & -1.2235390 & 0.0278127 \\
\hline & 3.3087987 & -0.8537570 & -1.0523853 \\
\hline & -3.1820642 & -2.9802194 & 1.4401666 \\
\hline & -2.3059349 & 5.0523187 & -0.1614878 \\
\hline & -4.0143643 & -2.7263046 & 2.1144066 \\
\hline & -2.9218200 & 1.7109554 & 0.1871723 \\
\hline & -2.9456243 & 6.4289715 & -0.3461016 \\
\hline & -2.3328455 & 0.4975896 & 0.0712432 \\
\hline & -3.9749229 & 6.4425586 & 0.0443494 \\
\hline & 1.1361435 & 2.7601600 & -0.7983161 \\
\hline & -1.2675199 & 5.0634542 & -0.5292684 \\
\hline & -2.9578978 & -1.8804262 & 0.4026047 \\
\hline & -2.5117681 & 2.6268777 & -0.7647424 \\
\hline & 1.3681381 & -2.6050740 & -1.4667298 \\
\hline & -3.4164055 & -3.9424624 & 0.9634418 \\
\hline & -3.0828164 & 3.9519537 & -0.8946071 \\
\hline & -1.4678635 & 0.3593605 & -0.7623467 \\
\hline & 1.0028910 & 0.1736180 & -1.8263551 \\
\hline & -2.1557283 & -2.1795692 & -0.2912085 \\
\hline & -4.1109984 & 3.9137163 & -0.5050282 \\
\hline & -2.9904264 & 6.7054623 & -1.4109427 \\
\hline & 1.5250796 & 1.0908231 & -2.1241261 \\
\hline & -0.4695869 & 0.2257159 & -1.9873420 \\
\hline & 1.4987604 & -0.6695514 & -2.3339712 \\
\hline & -3.8581278 & -1.7441502 & -0.2181182 \\
\hline & -1.8012213 & 2.3206475 & -1.4235529 \\
\hline & -3.1457862 & 4.2008321 & -1.9667450 \\
\hline & -0.7204711 & 1.1466132 & -2.541 \\
\hline
\end{tabular}




$\begin{array}{llll}\mathrm{H} & -0.8367055 & -1.9246037 & -2.1727792 \\ \mathrm{C} & -1.0686831 & -0.9849888 & -2.7028944 \\ \mathrm{H} & -2.1686539 & -0.9198526 & -2.7322629 \\ \mathrm{H} & -0.7231476 & -1.1254380 & -3.7477003\end{array}$




\section{List of Abbreviations}

\begin{tabular}{|c|c|}
\hline Abbreviation & Full Name \\
\hline $\mathrm{ACN}$ & Acetonitrile \\
\hline BJ & Becke-Johnson \\
\hline $\mathrm{BP}$ & Becke-Perdew \\
\hline $\mathrm{Bu}$ & Butyl \\
\hline B3LYP & Becke, 3-parameter, Lee-Yang-Parr \\
\hline cosmo & Conductor-like Screening Model \\
\hline COSMO-RS & Conductor-like Screening Model for Real Solvents \\
\hline COSY & Correlation Spectroscopy \\
\hline DCM & Dichloromethane \\
\hline DFT & Density Functional Theory \\
\hline DMSO & Dimethyl sulfoxide \\
\hline DOSY & Diffusion-Ordered Spectroscopy \\
\hline D3 & Atom-pair wise (atom-triple wise) dispersion correction \\
\hline EA & Elemental Analysis \\
\hline ESI & Electrospray Ionization \\
\hline eq. & Equivalents \\
\hline FIA & Flow Injection Analysis \\
\hline HMBC & Heteronuclear Multiple-Bond Correlation \\
\hline $\mathrm{HR}$ & High Resolution \\
\hline HSQC-ME & Heteronuclear Single-Quantum Correlation Multiplicity-Edited \\
\hline IS & Internal Standard \\
\hline LTQ & Linear Ion Trap Quadrupole \\
\hline $\mathrm{m}$ & Multiplet \\
\hline$m / z$ & Mass-to-charge ratio \\
\hline
\end{tabular}




\begin{tabular}{cl}
\hline Abbreviation & Full Name \\
\hline M & Molecular ion \\
MS & Mass Spectrometry \\
M06 & Minnesota 06 (functional) \\
$n$ & normal \\
NMR & Nuclear Magnetic Resonance \\
ppm & Part Per Million \\
Pr & Propyl \\
RI & Resolution-of-Identity \\
s & Singlet \\
SPS & Solvent Purification System \\
t & Triplet \\
TS & Transition State \\
TZVP & Valence Triple-Zeta Polarization \\
QZVP & Valence Quadruple-Zeta Polarization
\end{tabular}

